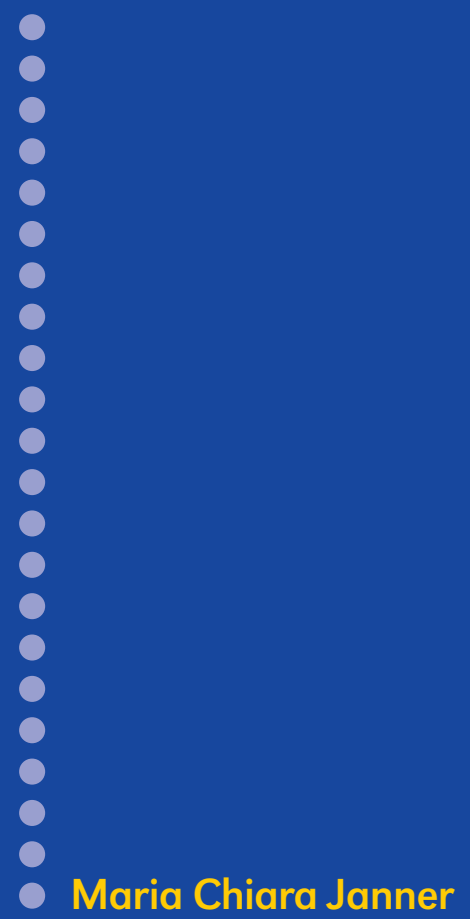

\title{
Sguardi linguistici sulla marca
}

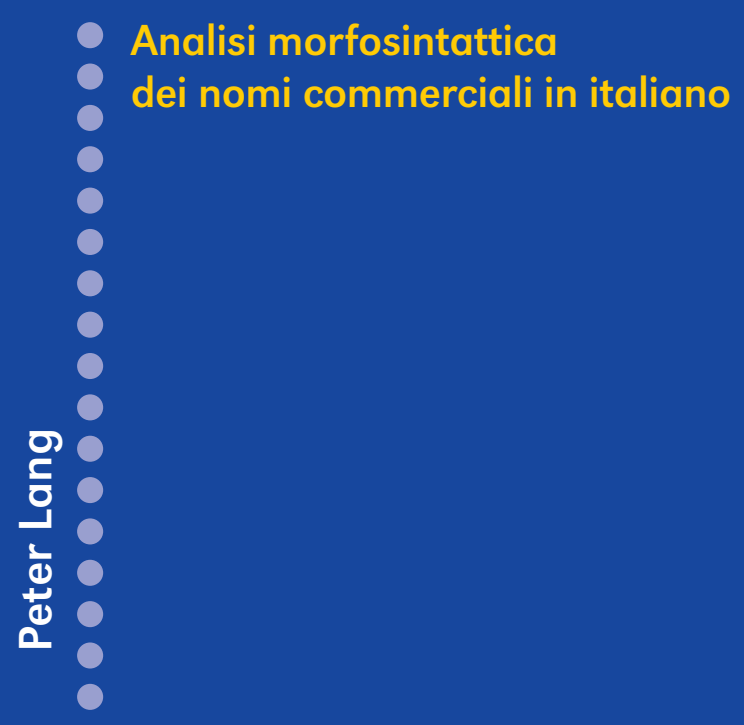


Coop, Voiello, Superga: nomi propri? nomi comuni? Come funzionano i nomi commerciali (o marchionimi) nella lingua, quali tratti presentano quando entrano nel discorso?

Il volume rappresenta il primo ampio studio sistematico sul funzionamento dei nomi commerciali nell'italiano scritto. Il suo carattere innovativo risie- de nell'impostazione sperimentale con cui indaga i marchionimi, scandagliando sintatticamente un vasto corpus di dati testuali appositamente elaborato. Prendendo ispirazione ideale dalla lezione di Saussure, i nomi commerciali sono processualmente descritti nei loro valori correlativi: non come entità ontologicamente predefinite, bensì come il risultato di rapporti sintagmatici e paradigmatici da cogliere metodologicamente nell'analisi delle proposizioni in cui tali nomi ricorrono. Ne emerge una visione radicalmente nuova del nome commerciale, scevra da luoghi comuni onomastici o categoriali, e in grado di gettare un fascio di luce, da una - prospettiva inconsueta, anche sul problema millenario dei nomi propri.

Dopo gli studi di lettere a Zurigo e a Roma, Maria Chiara Janner ha lavorato al progetto dottorale in linguistica italiana presso la KU Leuven e l'Universität Zürich UZH, dove è stata collaboratrice scientifi-

- ca. Insegna italiano nella scuola media superiore. La sua tesi di dottorato è stata insignita del "Premio Giovanni Nencioni 2016" attribuito dall'Accademia della Crusca. 
Sguardi linguistici sulla marca 


\section{Linguistic Insights}

Studies in Language and Communication

Edited by Maurizio Gotti, University of Bergamo

Volume 236

\section{ADVISORY BOARD}

Vijay Bhatia (Hong Kong)

David Crystal (Bangor)

Konrad Ehlich (Berlin / München)

Jan Engberg (Aarhus)

Norman Fairclough (Lancaster)

John Flowerdew (Lancaster)

Ken Hyland (East Anglia)

Roger Lass (Cape Town)

Matti Rissanen (Helsinki)

Françoise Salager-Meyer (Mérida, Venezuela)

Srikant Sarangi (Cardiff)

Susan Šarčević (Rijeka)

Lawrence Solan (New York)

Peter M. Tiersma (Los Angeles)

PETER LANG

Bern - Berlin · Bruxelles · New York · Oxford · Wien 
Maria Chiara Janner

\section{Sguardi linguistici sulla marca}

Analisi morfosintattica

dei nomi commerciali in italiano

Bern - Berlin - Bruxelles - New York · Oxford - Wien 


\section{Bibliografische Information Der Deutschen Bibliothek}

Die Deutsche Bibliothek verzeichnet diese Publikation in der Deutschen Nationalbibliografie; detaillierte bibliografische Daten sind im Internet über «http://dnb.ddb.de) abrufbar.

Die vorliegende Arbeit wurde von der Philosophischen Fakultät der Universität Zürich im Herbstsemester 2015 auf Antrag der Promotionskommission: Prof. Dr. Nunzio La Fauci, Universität Zürich UZH (hauptverantwortliche Betreuungsperson), und Prof. Dr. Elwys De Stefani, KU Leuven, als Dissertation angenommen.

Die Druckvorstufe dieser Publikation wurde vom Schweizerischen Nationalfonds zur Förderung der wissenschaftlichen Forschung unterstützt.

ISSN 1424-8689 hb.

ISBN 978-3-0343-2829-6 hb.

ISBN 978-3-0343-2831-9 EPUB
ISSN 2235-6371 eBook

ISBN 978-3-0343-2830-2 eBook

ISBN 978-3-0343-2832-6 МOBI

\section{PETER LANG}

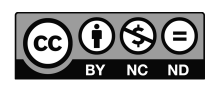

Open Access: Dieses Werk ist lizensiert unter der Creative Commons Lizenz Namensnennung - Nicht kommerziell - Keine Bearbeitungen 4.0 International (CC BY-NC-ND 4.0). Den vollständigen Lizenztext finden Sie unter: https:// creativecommons.org/licenses/by-nc-nd/4.0/deed.de

Diese Publikation wurde begutachtet.

(C) Maria Chiara Janner, 2017

Wabernstrasse 40, CH-3007 Bern, Switzerland

bern@peterlang.com, www.peterlang.com 


\section{Sommario}

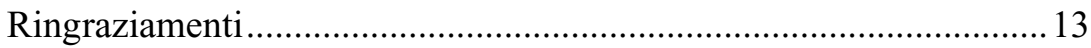

Una prospettiva diversa. Introduzione ................................................... 15

\section{Parte I. Stato della ricerca}

Introduzione 23

1. Dalla merce al desiderio. Cenni sullo sviluppo storico della marca.................................................................23

2. Riflessioni teoriche sul nome di marca:

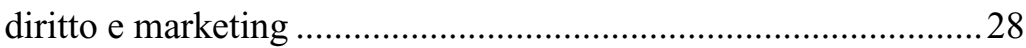

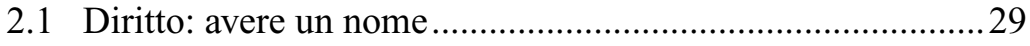

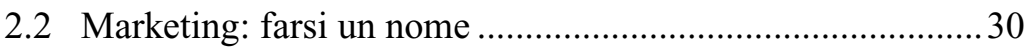

3. Linguistica: essere un nome. Aspetti teorici................................. 32

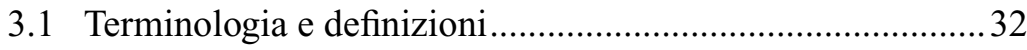

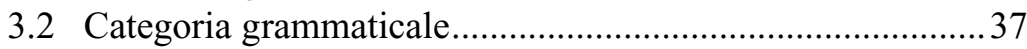

3.3 Evoluzioni nell'uso linguistico quotidiano............................ 41

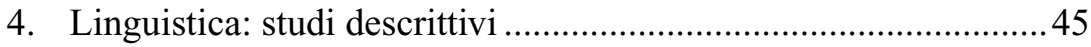

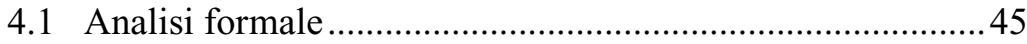

4.2 Marchionimi nelle opere lessicografiche............................47

4.3 Analisi contestuale ...........................................................5

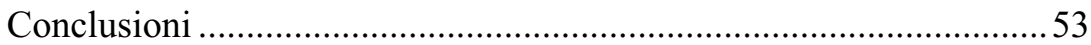

Parte II. Fondamenti teorici e metodologici

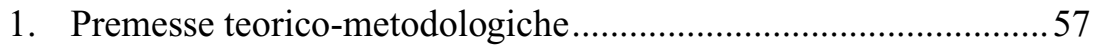

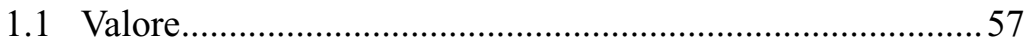

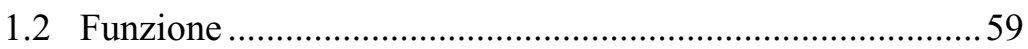


1.3 Studiare relazioni e ricercare opposizioni .............................60

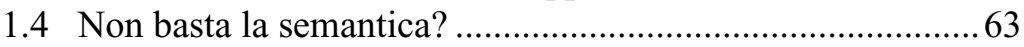

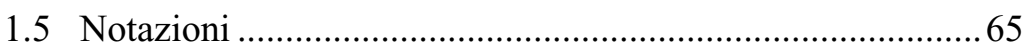

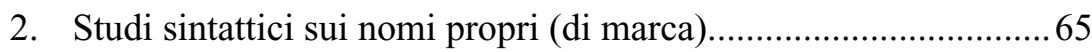

3. I dati: criteri di raccolta e problemi ............................................. 70

\section{Parte III. Analisi morfosintattica}

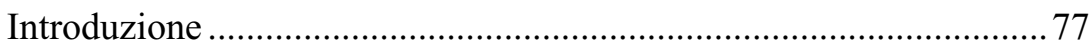

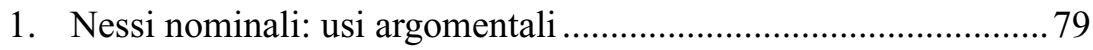

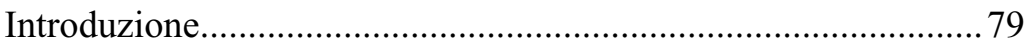

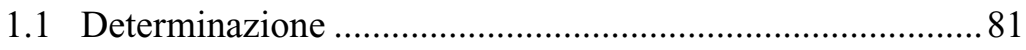

1.1.1 Determinatori sintatticamente motivati ....................81

1.1.2 Presenza e assenza di determinatori come varianti liberamente alternanti ........................8 87

1.1.3 Nomi commerciali sistematicamente determinati ......94

1.1.4 Ellissi o nominalizzazione? Il significato

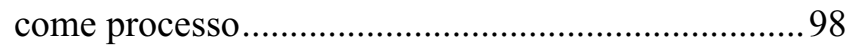

1.1.5 Riflessioni sistemiche sulla determinazione ........... 112

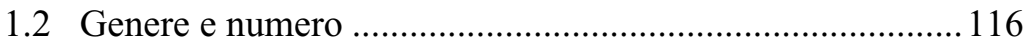

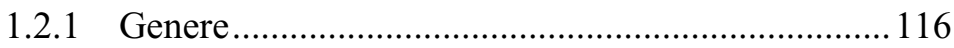

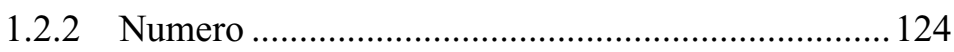

1.3 Modificazioni predicative .................................................... 127

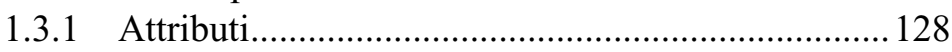

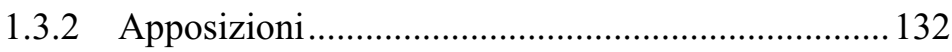

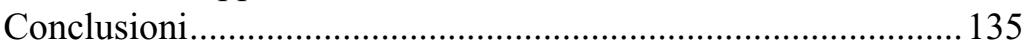

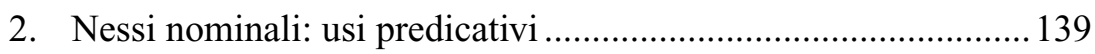

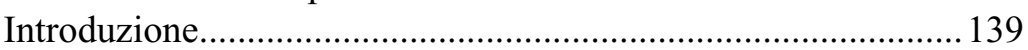

2.1 Marchionimo come predicato di denominazione ............... 143

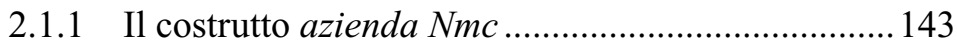

2.1.2 Il costrutto marca/marchio Nmc .............................. 152

2.2 Marchionimo come modificazione attributiva..................... 165 
2.2.1 Nessi $N$ Nmc con nomi concreti (relazione prodotto-produttore) ................................ 165

2.2.2 Nessi N Nmc con nomi astratti................................ 175

2.2.3 Nessi $N$ Nmc con nomi [+ umani].......................... 179

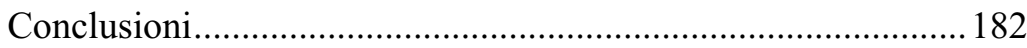

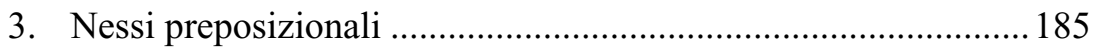

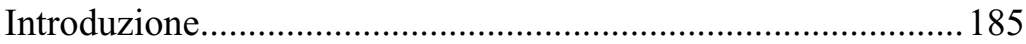

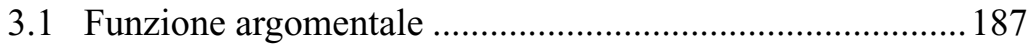

3.1.1 Nessi preposizionali con funzione argomentale nucleare .......................................... 187

3.1.2 Complementi preposizionali del predicato .............. 188

3.2 Modificazioni predicative: complementi di specificazione $(\mathrm{N} \mathrm{di} \mathrm{Nmc)}$.............................................. 193

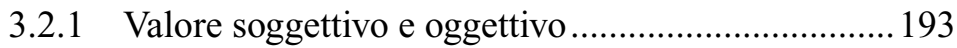

3.2.2 Relazione prodotto-produttore ................................. 198

3.2.3 Con nomi [+ umani] ................................................ 201

3.2.4 Relazione contenente-contenuto ............................205

3.2.5 Funzione di aggettivi relazionali...........................207

3.3 Altre tipologie di modificazioni predicative:

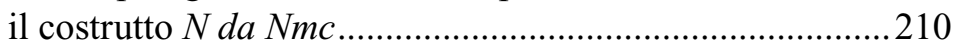

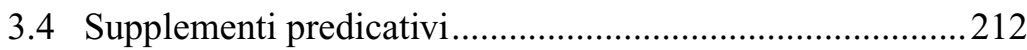

3.4.1 Supplementi locativi ............................................ 212

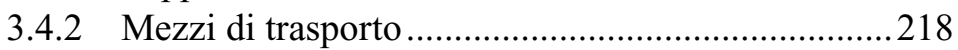

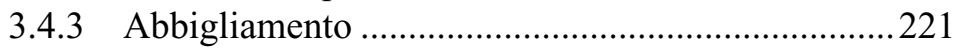

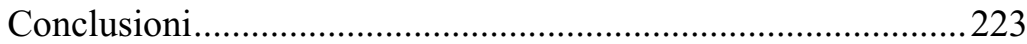

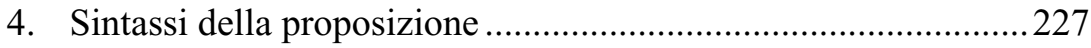

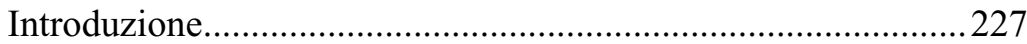

4.1 Funzione di soggetto e restrizioni di selezione..................229

4.2 Oggetti diretti in combinazione ricorrente

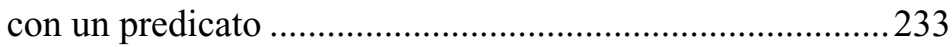

4.3 Costruzioni copulari ......................................................236

4.3.1 Nomi commerciali e predicato d'identità ...............236

4.3.2 Nomi commerciali in funzione predicativa.............238 
4.4 Volare Alitalia. Usi avverbiali.............................................. 240

4.4.1 Inquadramento diacronico ......................................240

4.4.2 Analisi funzionale: uso avverbiale o uso argomentale? ..................................................242

4.4.3 Interpretazione del costrutto ....................................2245

4.5 Quel cowboy targato Marlboro. Etichette

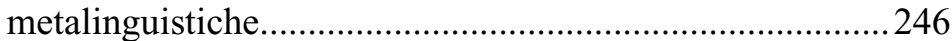

4.5.1 Aspetti predicativi della struttura...........................2246

4.5.2 Modificazione denominativa (con nomi riferiti a prodotti) ..................................248

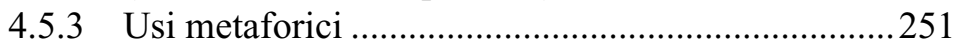

4.5.4 Non solo targhe, non solo firme. L'esempio di calzato ...............................................256

4.6 Alla Renault volevano Prost. Nessi preposizionali

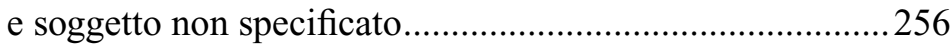

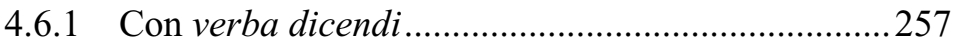

4.6.2 Con altri predicati verbali .......................................259

4.6.3 Osservazioni sulla struttura....................................260

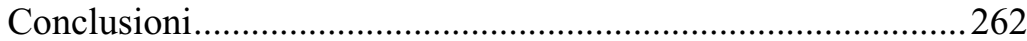

5. Barilliani o DeCecchisti? Derivazione e composizione.............265

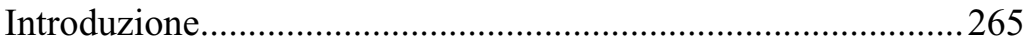

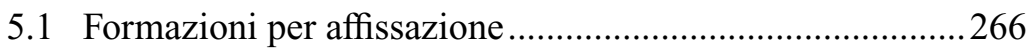

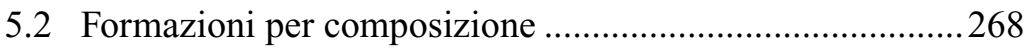

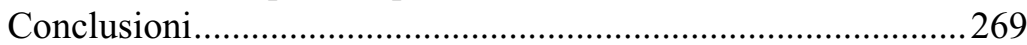

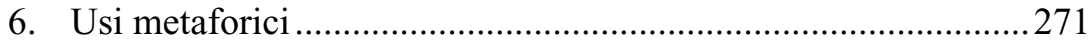

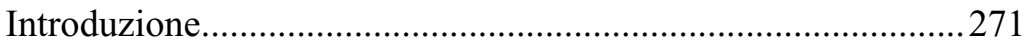

6.1 Marchionimi metaforici in funzione argomentale ..............273

6.1.1 Usi non modificati: Det Nmc ................................2273

6.1.2 Usi modificati: Det Nmc Mod .................................277

6.2 Marchionimi metaforici come modificatori ......................2280

6.2.1 Modificazione semplice: $N$ Nmc ............................2280

6.2.2 Con preposizione: $N$ da Nmc, $N$ a(Det) $N m c$..........2284

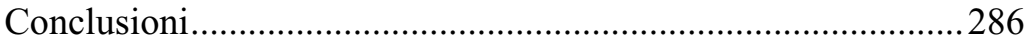


7. Dagli Avirex ai Miss Sixty in dodici anni. Marchionimi e narrativa: analisi di Federico Moccia, Tre metri sopra il cielo .............................287

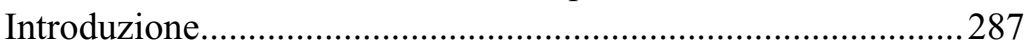

7.1 Usi argomentali e predicativi. Analisi linguistica................289

7.2 Sistema della moda, sistema della lingua.

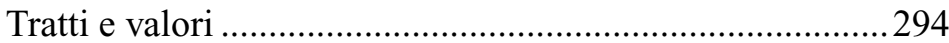

7.3 Le massaie di Migliorini e la nascita di luoghi comuni......296

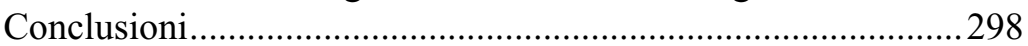

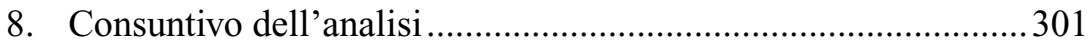

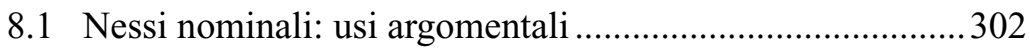

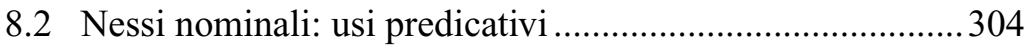

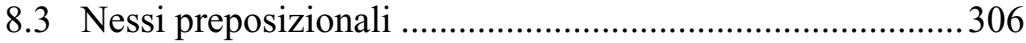

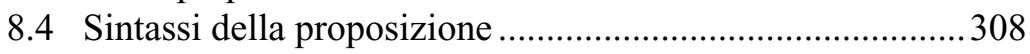

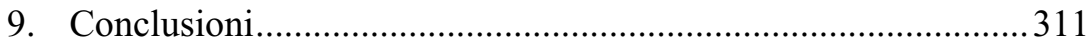

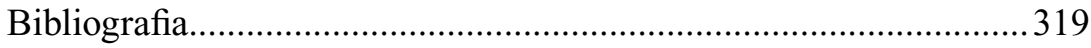

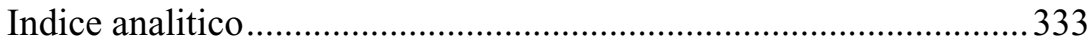

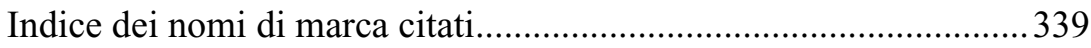



$\dot{\eta} \mu \varepsilon \tilde{\iota} \varsigma$ ớ $\delta \alpha \mu \varepsilon v$ ö $\tau 1 \mu \varepsilon \tau \alpha \beta \varepsilon \beta \eta \dot{\kappa} \kappa \alpha \mu \varepsilon v$

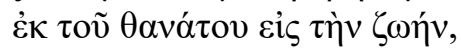

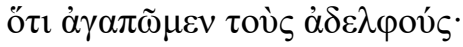

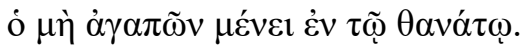

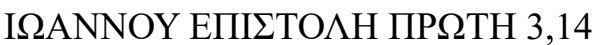

XLVII. Ogni esistente nella lingua è creato dalla relazione.

Nunzio La Fauci, Faccette di linguistica razionale 



\title{
Ringraziamenti
}

\begin{abstract}
Tra i miei familiari solo gli studi scientifici erano in onore; un mio zio materno era un chimico, professore universitario, sposato a una chimica (anzi ho avuto due zii chimici sposati a due zie chimiche[)]; mio fratello è un geologo, professore universitario. Io sono la pecora nera, l'unico letterato della famiglia. (Italo Calvino $)^{1}$
\end{abstract}

Provo simpatia per il Calvino che emerge da questo brano autobiografico, perché vi rivedo, fatta la tara di ovvie differenze, la mia situazione di letterata in mezzo a dentisti, matematici, farmacisti e fisioterapisti, per limitarmi a fratelli e sorelle. Proprio per questo - non "nonostante ciò" - primo destinatario di questo ringraziamento è la mia famiglia, che mi ha affettuosamente costretta a considerare con umiltà e con il necessario ridimensionamento l'attività affascinante e inutile di appassionata (e) indagatrice della lingua. Grazie, quindi, ai miei genitori Cristina e Fabio, a Simone e Martina, Gabriele e Patricia, Emmanuela e Pascale, per l'amore paziente con cui mi hanno accompagnata in questo cammino di maturazione scientifica e di crescita personale.

Gli anni dell'assistenza presso il Romanisches Seminar dell'Università di Zurigo sono costellati di innumerevoli memorie di momenti conviviali e discussioni arricchenti. Per questi, oltre che per i dolci e i caffè che abbiamo condiviso, sono profondamente grata a Camilla Bernardasci, Andrea Bonazzi, Alessandra Debanne, Rachele Delucchi, Vincenzo Faraoni e Lorenzo Filipponio.

Grazie a Lena Karssenberg per la sua amichevole presenza nei mesi febbrili di lavoro presso la KU Leuven e per la tenacia con cui ha tentato, purtroppo con scarsi risultati, di insegnarmi la pronuncia dei dittonghi neerlandesi.

Ringrazio il Programma dottorale di Romanistica dell'Università di Zurigo e in particolar modo la coordinatrice, Prof. Rita Catrina

1 In Ritratti su misura di scrittori italiani, a c. di Elio Filippo Accrocca, Venezia: Sodalizio del Libro, 1960, pp. 110-112, qui p. 110. 
Imboden, per le numerose occasioni, formali e informali, di crescita (trans)disciplinare e di rapporti con ricercatori giovani e affermati.

All'Accademia della Crusca, che ha onorato questa ricerca attribuendole il Premio Giovanni Nencioni 2016, va il mio sentito ringraziamento.

Infine, un cordiale ringraziamento ai professori Nunzio La Fauci ed Elwys De Stefani, che hanno accettato di seguirmi nell'elaborazione del progetto di ricerca: per gli innumerevoli suggerimenti, i commenti e le correzioni, per il costante incoraggiamento e per tutte le occasioni formative che mi hanno offerto, che rappresentano il risultato più prezioso di questo percorso. Ringrazio Elwys De Stefani per avermi aiutata nell'organizzazione del soggiorno presso la KU Leuven e per avermi accolta calorosamente in Belgio, consentendomi di compiere un'esperienza decisiva per lo sviluppo e il compimento del progetto. Ringrazio Nunzio La Fauci per la lezione su Saussure che ha tenuto or sono più di dieci anni, con la quale ha avuto inizio la mia passione per la lingua come relazione, nella consapevolezza che l'esistenza è valore correlativo. È la relazione tra le parti a creare, in un medesimo tempo, le parti e l'insieme: devo a lui, con gratitudine, l'ipotesi che sta a fondamento del presente lavoro.

Mendrisio, 11 agosto 2017 


\section{Una prospettiva diversa. Introduzione}

Si immagini un marziano che, giunto sul pianeta Terra, apre un quotidiano - nel caso specifico si tratta di un numero de La Repubblica - e vi legge queste righe:

La classe dirigente italiana veste Armani e Valentino, beve Chivas Regal, viaggia su Lancia (Thema), utilizza computer americani ( $\mathrm{Ibm}$ ) ed impianti hi-fi Pioneer. Il simbolo del successo sta ben stretto al polso: naturalmente firmato Rolex.

L'alieno, poniamo il caso, non è privo di abilità di decifrazione alfabetica e possiede qualche nozione di lingua italiana. Egli si sforza di comprendere il brano con l'ausilio di un dizionario ma, una volta giunto ad Armani, cominciano i problemi. Ha più fortuna rivolgendosi a un'enciclopedia, che gli permette di scoprire che Armani e Valentino sono i nomi di due stilisti e Lancia è una casa automobilistica italiana, cosicché la combinazione con vestire e viaggiare gli suona già più comprensibile; ma Pioneer lo manda, letteralmente, in tutt'altra direzione (peraltro più consona a lui, abituato a viaggiare nello spazio), mentre davanti a Chivas Regal il povero alieno deve arrendersi all'incomprensione.

L'avventura, però, non si arresta alla mera comprensione del testo. Una volta che ritiene di aver capito il messaggio, magari grazie ai più recenti mezzi di consultazione digitale, l'ambizioso marziano vuole passare dalle competenze passive a quelle attive; vuole, insomma, comunicare con gli italofoni inserendo nei suoi discorsi quelle parole dall'iniziale maiuscola che ha trovato nell'articolo del quotidiano e che, essendo dotato di spirito critico, vede ricorrere anche alla TV, su manifesti lungo la strada, sugli edifici e soprattutto sugli abiti, le vetture e gli alimenti dei Terrestri. Quali sono le caratteristiche linguistiche di tali nomi? Con quali altri elementi della lingua - nomi comuni, aggettivi, articoli, verbi ecc. - si combinano, e come? Ma qui, constata con rammarico l'alieno, non ci sono né grammatiche, né saggi che gli vengano in aiuto. 
Rolex, Pioneer, Lancia, Armani; inoltre Apple, Google, per certi versi anche Svizzera e, perché no, Pavarotti. La marca è fenomeno che ha caratterizzato antropologicamente il Novecento e, ragionevolmente, continuerà a caratterizzare ancora qualche tempo a venire. Nella marca, tratto saliente e rivelatore della civiltà occidentale moderna e postmoderna, convergono valori economici e principî ideologici, intenti comunicativi e istanze identitarie, specificazioni giuridiche e definizioni ontologiche, nell'interfaccia tra qualità simboliche e beni reali. Tutto ciò è racchiuso in una parola che presenta caratteristiche non dissimili da un tipo di parole in particolare: quelle che, prima di predicare qualcosa (o addirittura senza predicare nulla), denominano e, senza classificare o prima di classificare, individuano ciò che denominano. I nomi propri, insomma. La marca, di conseguenza, è materia di riflessione linguistica. Nel riflettere sulla marca, però, com'è avvenuto e avviene per i nomi propri negli studi onomastici, tradizionalmente considerati un parente povero della linguistica (cf. Molino 1982: 5), la linguistica ha spesso rinunciato alla sua autonomia dottrinale; dimenticandosi di essere una disciplina sperimentale, oltre che, inscindibilmente, speculativa, ha cercato definizioni e indirizzi procedurali nel pensiero dei filosofi, in particolare nella riflessione dei logici. Pochi esempi, per giunta inventati allo scopo, sono stati girati e rigirati nel tentativo di cavarne l'essenza della categoria. Discussioni interminabili, ipotesi fondate più sulle interpretazioni degli studiosi che su osservazioni sperimentali, hanno condotto all'oblio la semplice procedura che Ferdinand de Saussure assegnò alla linguistica: nella lingua, il valore di qualsiasi entità è dato dal rapporto che permette di individuarla nella prospettiva delle relazioni in praesentia, dette sintagmatiche, e correlativamente in quella delle relazioni in absentia, da Saussure dette associative e in seguito paradigmatiche.

Sulla scorta di tali indicazioni di metodo, l'analisi condotta in queste pagine intende formulare un'ipotesi di risposta agli interrogativi cui allude la vicenda del marziano delineata in apertura: in che modo i marchionimi, ossia nomi propri legati al mondo del commercio (cf. Bergien, Kremer \& Zilg 2008), ricorrono nel discorso, nel caso specifico dell'italiano? Quali sono le loro proprietà morfosintattiche? Con quali elementi si combinano negli enunciati? Con quali conseguenze a livello di significato? L'invito è, quindi, ad adottare un punto di vista 
alieno - etimologicamente: a guardare ai marchionimi da una prospettiva insolita, non quella di chi, in quanto consumatore e in quanto parlante, è abituato a vederli e inserirli quotidianamente nella sua comunicazione ma di chi, quasi dall'esterno, li considera come elementi del sistema lingua e cerca di comprenderne la natura, che si esplica in usi testuali molteplici e variegati.

Ricalcando le formule del marketing, questo lavoro si potrebbe definire uno studio di brand integration in prospettiva linguistica, una riflessione sull'inserimento delle marche nel discorso. La sua forza innovativa consiste nell'impostazione sperimentale con cui indaga $i$ marchionimi in prospettiva testuale e sintattica, considerando gli usi effettivi da parte degli utenti della lingua con un'attitudine puramente descrittiva. I nomi commerciali vi sono considerati nella loro natura processuale: non come entità ontologicamente predefinite, bensì come il risultato di relazioni sintagmatiche e paradigmatiche che è possibile cogliere grammaticalmente studiando le proprietà combinatorie che tali nomi, di volta in volta, manifestano all'interno della proposizione.

Obiettivo primario dello studio è descrivere come funziona il nome di marca nel discorso scritto, fornendo una panoramica delle strutture in cui ricorre e delle proprietà combinatorie che manifesta. La ricerca, insomma, intende elaborare il capitolo "Morfosintassi" di un'ideale grammatica dei marchionimi, della quale esistono già le parti dedicate a fonetica e morfologia; intende offrire alcuni spunti non per un dizionario dei nomi commerciali, quanto piuttosto per un ipotetico tesauro.

Lo studio delle relazioni distribuzionali tra elementi (cf. Harris 1952: 5) permette di differenziare usi diversi, quindi valori diversi, dei marchionimi. Sarà così possibile approfondire la questione del loro significato; un problema spinoso, dal momento che si tratta di nomi propri e che, fatto ancor più importante, sono frequenti i casi di omonimia, dove alla variazione di valore non corrisponde una variazione formale (la Fiat come impresa e come automobile). Il nome commerciale che galleggia alla superficie della lingua è semplice e univoco soltanto in apparenza: esso cela infatti un processo che è tanto più difficile da determinare quanto meno è evidente (cf. La Fauci 2011: 51). Attraverso lo studio contestualizzato dei nomi commerciali - nomi propri, data la 
loro natura predicativa, ma al contempo nomi propri critici - si intende proporre inoltre un'ipotesi sulla natura linguistica del nome proprio, passando da una definizione categoriale a una processuale. Si tratta insomma di destrutturare il concetto di nome proprio come categoria per riformularlo come processualmente determinato.

La ricerca che qui si propone è fondata su una concezione relazionale e funzionale della lingua, di stampo saussuriano (cf. La Fauci 2011). Essa prende avvio da un concetto intuitivo di "nome commerciale" senza stabilirne una definizione a priori. La questione terminologica, del resto, è tuttora aperta; nel corso della ricerca si utilizzeranno i termini "nome di marca", "marchionimo" e "nome commerciale" come sinonimi, pur essendo consapevoli che non sono perfettamente sovrapponibili.

Fondamentale criterio di metodo è l'adesione al testo. Si procede ricercando opposizioni pertinenti e regolarità sistemiche nell'uso di marchionimi, nell'intento di stabilire dipendenze reciproche e processi di costituzione di valori. In particolare si ipotizza che non siano i significati a motivare la presenza di eventuali articoli, di particolari tratti di genere o numero e così via ma che, viceversa, siano i contesti di ricorrenza a far emergere valori specifici del nome di marca. Differenze di ordine semantico, come quelle messe in luce da Van Langendonck (2007a: 235-238; cf. Parte II, cap. 2), sono pertanto secondarie rispetto alle differenze formali che consentono di cogliere le proprietà correlative manifestate da nessi e proposizioni. Questo ribaltamento di prospettiva è legittimato da una circostanza facilmente osservabile: nei testi - si pensi al brano de LaRepubblica che apre il lavoro - i nomi di marca ricorrono in combinazioni diverse e presentano varie funzioni. Per questo si ritiene più fruttuoso un avvicinamento non semantico ma sintattico, non ontologico ma processuale, che sia in grado di comprendere, dietro e attraverso le apparenze morfosintattiche, il fenomeno linguistico dei nomi di marca non come un fatto, bensì nel suo farsi. La varietà funzionale manifestata dai nomi di marca è espressione della duttilità della lingua e della sua natura relazionale.

Il metodo analitico proposto implica la necessità di disporre di dati di tipo testuale, non di semplici liste di nomi. Si è pertanto provveduto a raccogliere un insieme di dati con esempi di testualità in cui 
ricorrono marchionimi (testi giornalistici: quotidiani, riviste per consumatori; opere di narrativa). I costrutti preparati per l'analisi si attestano a un totale di circa $2^{\prime} 400$, una parte dei quali (quasi ottocento) sono riportati nei capitoli che seguono. Procedendo per campioni, di marchionimi e di testi, l'analisi resta a ogni modo qualitativa, non quantitativa, e non ha alcuna pretesa di rappresentatività, se non per i singoli esempi considerati. In generale si sono preferiti marchionimi con un maggior grado di prototipicità, per es. Barilla o Vespa, per i quali si può prevedere un più alto grado di chiarezza definitoria rispetto a nomi come Banca Carige o I sapori dell'orto.

Il lavoro è strutturato in tre parti. La Parte I (Stato della ricerca) presenta una panoramica sulla bibliografia relativa ai marchionimi in linguistica e in altre discipline (diritto, marketing, economia). La Parte II (Fondamenti teorici e metodologici) fornisce indicazioni di metodo e sui criteri di raccolta ed elaborazione dei dati. Il cuore del lavoro è costituito dalla Parte III (Analisi morfosintattica), strutturata secondo i contesti sintattici di ricorrenza dei marchionimi. L'analisi affronta innanzitutto i nessi (o sintagmi) nominali, distinguendo tra i costrutti in cui il marchionimo svolge funzione argomentale, di nucleo del nesso, e quelli in cui svolge funzione predicativa, come modificatore o attributo di un altro nome. Sono poi analizzati diversi tipi di nessi preposizionali e, a seguire, le funzioni manifestate dai nomi commerciali a livello di proposizione. I tre capitoli successivi si concentrano su aspetti correlati all'analisi sintattica: derivazioni morfologiche su base marchionimica; usi metaforici dei marchionimi; infine, in una prospettiva più ampia, si propone l'analisi sintattica dei nomi commerciali ricorrenti in un'opera di narrativa recente, Tre metri sopra il cielo di Federico Moccia. 

Parte I

Stato della ricerca 



\section{Introduzione}

Le pagine che seguono tratteggiano una panoramica della marca così come ricorre nella bibliografia: evoluzione storica $(\S 1)$, aspetti giuridici e di marketing (\$2) e, in prospettiva linguistica, studi sulla determinazione categoriale (§3) e studi descrittivi (§4). Tra questi, una particolare attenzione è data alla tipologia di ricerche che, per il loro taglio metodologico, sono più affini al punto di vista qui adottato: quelle - poche che si rivolgono a dati testuali con l'obiettivo di studiare i marchionimi nel discorso, da un punto di vista combinatorio.

La marca è un "soggetto multidimensionale" (Minestroni 2010: 14) che comporta diverse faccette oltre a quella nominale, da quella visiva a quella sonora. Pur essendo consapevoli che "una vera marca è sempre più di un semplice nome" (Semprini 1996: 43), in questa sede ci si concentra su di esso, in quanto pertinente agli studi linguistici.

\section{Dalla merce al desiderio. Cenni sullo sviluppo storico della marca}

"Everything is a brand", scrive Kotler (2003: 8): "A brand is any label that carries meaning and associations". Non solo Coca-Cola, quindi: sono marche anche New York City, Greenpeace e David Beckham (cf. Adjouri 2014: 30; Lury 2004: 16). Da un altro punto di vista, la marca è "il processo discorsivo che, mettendo in relazione cose e valori, li genera come entità indipendenti" (Marrone 2007: 4); si tratta dunque di un'istanza semiotica (Semprini 1992: 27). ${ }^{1}$ Minestroni (2010: 133)

1 È ancora Marrone a sottolineare la necessità di analizzare le marche da un punto di vista semiotico (v. Mangano \& Marrone 2015: 46). Il volume in cui è contenuto il saggio (Rossolatos 2015) si propone come manuale di riferimento per la ricerca sulle marche nel settore della semiotica. 
la paragona al segno di Saussure (o piuttosto della vulgata saussuriana) in quanto "istanza formata dall'unione di due entità intangibili", associate arbitrariamente: ${ }^{2}$ da un lato vi sono nome, slogan, simbolo, alla stregua del significante; dall'altro le associazioni che la marca suscita, i benefici che promette, la sua personalità, che ne costituiscono il "significato" (Minestroni 2010: 134). ${ }^{3}$ Per Adjouri (2014: 32) la marca si compone di due livelli: percettibile/non-percettibile (wahrnehmbar/ nicht-wahrnehmbar); la rappresentazione grafica che propone per illustrarlo ricorda - forse non per caso - l'ellissi divisa a metà del Cours saussuriano.

Gli studiosi, pur faticando a fornire una definizione precisa della marca in ragione della sua natura complessa (cf. §3.1), sono concordi nell'attribuirle un carattere inter- e multidisciplinare (Adjouri 2014: 28-29) e nel considerarla elemento di un sistema multidimensionale (Semprini 1992: 28), funzionalmente integrato in tre universi: economia, diritto e lingua (Teutsch 2007: 19).

Lungi dall'essere fine a se stessa, la marca ha una natura essenzialmente relazionale (cf. Teutsch 2007: 48; Lury 2004: 2; Minestroni 2010: 96). Si inserisce infatti nell'ambito della relazione tra produttore, commerciante e consumatore, costituendosi allo stesso tempo in funzione di un produttore (marca come indice di appartenenza) e di prodotti in apparenza simili ma non uguali (marca come strumento di differenziazione). ${ }^{4}$ Dove si instaura una relazione, emergono differenze: la marca è appunto "l'indicazione scritta di una differenza" (Botton, Cegarra \& Ferrari 2002: 45). L'operazione di differenziazione può

2 In realtà si può mettere in dubbio che si tratti di un'unione arbitraria, soprattutto dal punto di vista del marketing.

3 La presenza di un "significato" della marca, comunque si voglia intendere questo concetto - peraltro dibattuto -, non può essere ignorata. Di fronte all'affermazione di Teutsch (2007: 135) per il quale le marche, in quanto etichette, sono interscambiabili ("alle Marken sind Synonyme"), viene da chiedersi quanto riuscirebbero a vendere un caffè Mastro Lindo o un lucido per scarpe Nestlé.

4 Lury (2004: 27) suggerisce che le definizioni storiche della marca sono insufficienti per quella odierna, che può essere descritta come "the organisation of a set of multi-dimensional relations between products or services". L'essenza della marca è la relazione: "it is their relation to one another that makes the components of a car into a car" e lo stesso vale per la marca (Lury 2004: 2). 
comportare, come conseguenza, lo stigma di un giudizio qualitativo: "Marcare vuol dire al contempo distinguere e valutare, segnalare alla stima o alla disapprovazione" (Botton, Cegarra \& Ferrari 2002: 45).

Identificazione (del produttore) e differenziazione (di un prodotto rispetto ad altri) sono le due funzioni primarie della marca (cf. Lindemann 2010: 7), che gli studiosi riconoscono tanto alle sue forme più antiche - la marchiatura di bestiame e vasellame - quanto al branding moderno. ${ }^{5}$ Semprini (1996: 28-32) annovera tra le funzioni fondamentali della marca originaria anche quelle di appropriazione (ogni intermediario appone il suo marchio per far valere un diritto di proprietà sull'oggetto) e di qualificazione, che rappresenta un tratto di modernità dei "proto-marchi": se, per es., con il passare del tempo i trasportatori notavano che le terrecotte di una data bottega erano più resistenti o consegnate con puntualità, la marca che le contrassegnava consentiva di riconoscere una qualità (o un difetto) del prodotto e del contesto commerciale. Il marchio imposto sul vasellame o sui capi di bestiame permetteva di dedurne rapidamente caratteristiche qualitative e di prezzo, facilitando il processo di scelta e acquisto. La marca diveniva così, in nuce, garanzia della presenza o assenza di un valore. "Lo scarabocchio diventa segno: consente di conoscere le qualità e i difetti di un prodotto senza doverne fare l'esperienza" (Semprini 1996: 30).

La marca, dunque, è doppiamente inscritta nel tempo, filogeneticamente e ontogeneticamente: sia come fenomeno culturale in continua, secolare evoluzione, sia come entità individuale in grado di acquistare un "buon nome" col passare degli anni. Quanto allo sviluppo filogenetico, va però osservato che il concetto di marca di Etruschi, Greci e Romani, ${ }^{6}$ malgrado condivida con la marca moderna alcune caratteristiche fondamentali, è profondamente diverso.

5 A queste funzioni si aggiungono, secondo Kapferer \& Thoenig (cit. in Minestroni 2010: 96-102), quelle di orientamento (strutturare l'offerta), personalizzazione (scelta di marche come agire sociale), garanzia (marca come promessa di qualità), ludica (il piacere che si prova nel selezionare il proprio prodotto tra altri), di praticità (la marca è la memoria del prodotto: fa risparmiare tempo e semplifica la vita; cf. Kotler 2003: 9).

6 O di popoli ed epoche ancora più antichi: Lury (2004: 166n) afferma che "some form of branding may be said to have existed for almost five thousand years". 
Una prima differenza consiste nel fatto che le marchiature antiche erano rivolte non tanto al destinatario ultimo del prodotto, bensì agli intermediari tra produttore e acquirente (distributori, venditori); per i consumatori, infatti, "tutte le terrecotte sono grigie" (Semprini 1996: 29). Al contrario, la marca moderna, nata a fine Ottocento ${ }^{7}$ (cf. Semprini 1996: 32; Teutsch 2007: 23), svolgeva e svolge la funzione essenziale di orientare i potenziali acquirenti, superando il divario sempre più ampio tra le due istanze del produttore e del consumatore: "Facilitating choice is probably the most important purpose of branding in commerce" (Lindemann 2010: 3). In un'epoca di forte standardizzazione qualitativa nacque l'esigenza non solo di identificare prodotti simili come appartenenti a diversi produttori, ma di differenziarli più di quanto non lo fossero realmente (o sensibilmente) per aiutare i consumatori nella scelta. ${ }^{8}$

Un'altra differenza: verso gli anni Quaranta del Novecento, la marca si allargò a comprendere aspetti non più soltanto legati a caratteristiche qualitative e funzionali dei prodotti, ma anche psicologici ed emotivi, divenendo un veicolo di prestigio sociale, un coagulo di sogni. Dal momento che la qualità dei prodotti presentava di regola ormai poche differenze, le aziende cominciarono a puntare sui "vantaggi

7 In questo periodo emergono anche le prime leggi sulle marche. La regolamentazione giuridica è un tratto fondamentale che differenzia la marca moderna da fenomeni simili di epoche precedenti.

8 Esperimenti di psicologia della percezione nell'ambito del gusto hanno mostrato come la presenza di marche diverse accentui le differenze percepite tra prodotti simili o addirittura uguali. Hoegg \& Alba (2007a; 2007b) offrono il resoconto di due esperimenti con succhi d'arancia modificati in maniera percepibile quanto al grado di dolcezza; differenze (fittizie) di marca ingannavano la percezione sensoriale del gusto nei soggetti del test, che valutavano come "più diversi" gusti in realtà uguali ma contrassegnati con marche distinte, e viceversa. Come mostra inoltre il noto Pepsi Challenge, la sfida a distinguere Coca-Cola e Pepsi in un assaggio alla cieca, la personalità della marca "è talmente forte da falsare il gusto della bevanda" (Minestroni 2010: 222).

Tra i consumatori regna dunque "la convinzione (e la percezione) che esistano diversità tra marche concorrenti all'interno di una stessa categoria merceologica" (Minestroni 2010: 190), convinzione che prescinde dall'esperienza reale di un prodotto e a volte, come nel caso del gusto, riesce persino a falsarla. Capita così che la marca (di successo, bisogna aggiungere) diventi "un pregiudizio" o, in altri termini, "una sorta di valore aggiunto" (Minestroni 2010: 220). 
emozionali" collegati alla marca (cf. Lindemann 2010: 4). Le attività di marketing scoprirono le dimensioni sociali, psicologiche e antropologiche della relazione tra il consumatore e la marca. Nell'epoca del "consumo di massa", libero dagli stigmi dell'etica protestante, "[1]a gente inizia a chiedere - per la prima volta - unicità, distinzione e stile: vuole oggetti capaci di appagare i desideri, oltre che i bisogni primari" (Minestroni 2010: 44).

Durante le fasi di questo sviluppo emerge la natura narrativa della marca - sistema simbolico, concentrato di immagini e valori, incarnazione del desiderio -, che sarà sviluppata nei decenni a venire e a tutt'oggi non ha perso d'attualità, benché i metodi di comunicazione della marca, con l'avvento delle nuove tecnologie, siano mutati, divenendo più raffinati e pervasivi. Si impone pertanto una politica di marketing che tenga conto del "nuovo statuto epistemologico - eminentemente narrativo - della marca, con lo spessore dei significati che questa oggi deve veicolare" (Fabris 2010: 7). Non sorprende che sia un fenomeno al contempo linguistico (un nome) ed economico ad aver preso il posto dei grands récits (Jean-François Lyotard) nell'epoca postmoderna. La marca, dunque, si è allontanata dalla sua antenata di epoca industriale e ancor più da quella originaria, in cui identificazione del produttore e differenziazione del prodotto dal resto dell'offerta erano le funzioni principali.

Tuttavia, per una ricerca linguistica come la presente, la differenza fondamentale tra $\mathrm{i}$ fenomeni di marchiatura antichi e la marca moderna risiede nel fatto che in quest'ultima è centrale il nome, la parte linguistica della marca. Solo per la marca moderna vale quanto scrivono Ainiala, Saarelma \& Sjöblom (2012: 213): "A name is the core of a brand". Nel corso del Novecento, la parte nominale della marca è andata acquistando predominanza rispetto al segno grafico, storicamente più antico $^{9}$ (cf. Botton, Cegarra \& Ferrari 2002: 52). Oltre a mutare le modalità di comunicazione della marca, ciò rende possibile non solo il parlare delle marche ma anche il parlare con le marche, inserendo i nomi di marca nel discorso. Il marketing scopre un nuovo

9 Lindemann (2010:3) riporta un esempio risalente alla prima epoca industriale: le porcellane di Meissen, marchiate dal 1722 con il disegno di due spade incrociate. 
obiettivo oltre a quello, classico e intramontabile, di vendere un prodotto: far sì che esso sia conosciuto e riconosciuto, faccia parlare di sé e assurga in tal modo a oggetto del desiderio (si pensi al concetto di brand awareness, che misura la notorietà di una marca). In questo il ruolo del nome della marca è centrale.

Soggetto della comunicazione con la marca sono non solo le imprese ma anche i consumatori, che acquistano un ruolo attivo in quanto sono anche, anzi prima di tutto, dei parlanti. Nei loro discorsi, $\mathrm{i}$ nomi di marca - creati in laboratorio, per così dire, e inseriti nel panorama linguistico attraverso pubblicità e affini - diventano parte viva della lingua, sono ri-creati, mutano di significato; capita persino che entrino nei dizionari, i quali ne consacrano l'appartenenza al lessico di una o più lingue (cf. $\$ 3.3$ e 4.2).

La ri-creazione della marca nel discorso ne sottolinea inoltre l'aspetto ludico, il piacere che si prova a mettere in gioco, parlando, conoscenze condivise entro un gruppo più o meno ampio: dire il Folletto invece di aspirapolvere o lo Scottex per carta da cucina è forse più pratico, ma soprattutto istituisce uno spirito di koinè. È il piacere delle antonomasie, dei luoghi comuni del lessico familiare ancor prima che regionale.

\section{Riflessioni teoriche sul nome di marca: diritto e marketing}

Conformemente alla natura multidimensionale della marca, sono numerose le discipline che se ne sono occupate: Adjouri (2014: 28-29) elenca economia, marketing, giurisprudenza, psicologia, sociologia, design, semiotica, scienze della comunicazione.

Il fatto che la lista di Adjouri non comprenda la linguistica è sintomatico di un aspetto che gli studi e le rassegne bibliografiche non mettono sufficientemente in evidenza. Le discipline elencate non mirano solo a studiare la marca nella sua globalità ma sono anche e soprattutto tributarie di una prospettiva strettamente funzionale, ossia 
considerano la marca in quanto strumento che svolge precise funzioni in ambito economico e giuridico. Tali studi, inoltre, hanno non di rado una finalità pratica, offrendo criteri per l'elaborazione giuridicamente corretta delle marche, per la creazione di marche di successo e per una comunicazione efficace delle stesse. La linguistica, invece, è essenzialmente descrittiva, non normativa o funzionale ad aspetti commerciali.

I paragrafi seguenti sono dedicati alla bibliografia sui nomi di marca nei due ambiti fondamentali del diritto (\$2.1) e del marketing (§2.2).

\subsection{Diritto: avere un nome}

Le definizioni tradizionali della marca in ambito giuridico sono incentrate sulle funzioni distintiva e identificativa (cf. Lindemann 2010: 5): "A trademark is a distinctive word, phrase, logo, graphic symbol, or other device that is used to identify the source of a product or service and to distinguish it from competitors" (Stim 2007: 344).

La denominazione di un'azienda o di un marchio è un atto giuridicamente regolato e che sottostà a direttive particolari, solitamente diverse per "marchi" e "ditte", perché al nome sia garantita la protezione legale. Quest'ultima trova la sua ragion d'essere nel fatto che il nome di marca è un "moltiplicatore di valore" (Minestroni 2010), per cui un abuso del nome da parte di altre istanze può comportare perdite finanziarie.

Il diritto è costitutivo del nome di marca sin dal suo nascere; esso regola gli aspetti legali connessi alla necessità, per i marchi e le imprese, di avere un nome, non diversamente da quanto avviene per le persone fisiche (cf. Van Langendonck 2007b: 26). Non tutti i nomi, infatti, sono "markenfähig" (Teusch 2007: 10), ossia potenzialmente in grado di fungere da marchionimi. I principî base da rispettare sono $\mathrm{i}$ seguenti: il nome - sia della marca, sia della ditta - deve avere carattere distintivo; non deve confondere gli utenti; non deve trarli in inganno (cf. Ballon 2007: 67-68; Koss 1996b: 1796-1797 e 1800; Solly 2002: 220).

La protezione legale della marca è sempre in funzione del prodotto (merci o servizi) che essa contrassegna (cf. Teutsch 2007: 68). Ne deriva una restrizione fondamentale: i nomi di marca non possono essere 
descrittivi del prodotto. Si tratta di una questione semantica in senso ampio (Teutsch 2007: 10-11): per chiarire la registrabilità di un nome è necessario considerare la relazione tra il significato del contrassegno che si vuole apporre al prodotto e il significato del nome di tale oggetto. "APFEL" non può essere registrato per un succo di mela; per un modello di computer invece sì (Teutsch 2007: 12-13). L'eventuale esclusione dal registro dei marchi dipende pertanto dall'ambito di protezione, dai beni e/o servizi contraddistinti dalla marca (Teutsch 2007: 26).

Se una marca non può essere descrittiva dal punto di vista giuridico, essa deve esserlo - per altri versi - nella prospettiva economica: su questo sottile confine nascono i conflitti tra proprietari del marchio e autorità (cf. Teutsch 2007: 27). Se non attraverso una significazione diretta ed esplicita, il nome di marca cercherà di farsi strada nella memoria del consumatore attraverso aspetti connotativi, per es. sfruttando il significato delle basi lessicali riutilizzate nella creazione del nome (cf. Petit 2006: 695; Teutsch 2007: 103-104).

Mentre prodotti dissimili possono portare lo stesso nome, per es. San Pellegrino/Sanpellegrino per bevande e calze (un caso di omonimia legale: cf. Botton, Cegarra \& Ferrari 2002: 70), nello stesso settore merceologico non è permesso registrare nomi uguali o tanto simili da poter indurre in confusione i consumatori. Spetta anche ai linguisti stabilire il grado di somiglianza tra due nomi e quindi un eventuale plagio (o "Markenpiraterie", Teutsch 2007: 109n); si veda il caso di uno sciroppo per la tosse denominato Damylin, accusato di imitare nel nome l'originale Calmylin (Leblanc 2008).

\subsection{Marketing: farsi un nome}

Se è vero, come afferma Kotler (2003: 8), che tutto è una marca, da Madonna al lettore delle sue pagine, va altresì ricordato che non tutto può essere una marca in senso stretto: dal profilo legale - lo si è appena visto - non tutti i nomi sono registrabili e dal profilo commerciale vi sono casi in cui farlo sarebbe svantaggioso, benché legalmente possibile. Si pensi ai big business blunders, nuove marche rivelatesi fallimentari a causa di assonanze e allusioni disforiche in altre lingue, come 
Chevrolet Nova in Paesi ispanofoni o il ferro per capelli Mist Stick sul mercato tedesco (cf. l'elenco di casi riportato in Platen 1997: 155-159).

Nella prospettiva del marketing convergono due aspetti principali: 1) il naming, la creazione del nome, da parte o su commissione dell'impresa; 2) la ricezione del nome, in particolare gli usi linguistici dello stesso e le direttive volte a regolarli al fine di evitare danni economici alla ditta. A una marca infatti non basta avere un nome, registrato e protetto; chi ne è proprietario vuole che essa abbia successo, sia conosciuta, incrementi le vendite - insomma, la marca deve farsi un nome (cf. Botton, Cegarra \& Ferrari 2002: 48; Minestroni 2010: 24). A questo scopo è necessario sia partire con il nome giusto, sia coltivarne la crescita e controllarne gli sviluppi, anche linguistici (per i quali v. §3.3).

Con il naming, il nome di marca si fa esso stesso prodotto (cf. Botton, Cegarra \& Ferrari 2002: 40): agenzie specializzate, che si avvalgono della collaborazione di linguisti (cf. Teutsch 2007: 62), vengono in aiuto alle ditte nella complessa impresa di inventare - anche etimologicamente - il nome giusto per una marca, ossia "quello che anticipa e annuncia la bontà del prodotto" (Botton, Cegarra \& Ferrari 2002: 49). Esso "rappresenta la memoria del prodotto stesso, e una volta scelto è molto difficile e costoso da cambiare" (Botton, Cegarra \& Ferrari 2002: 9).

La difficoltà risiede nel trovare nomi che sappiano farsi notare nell'oceano di marche in cui sono immersi i consumatori, siano evocativi (alludendo senza significare direttamente), si possano utilizzare internazionalmente, ${ }^{10}$ siano distintivi e possano ricevere protezione legale (cf. Botton, Cegarra \& Ferrari 2002: 107-108; Teutsch 2007: 63). Da un profilo latamente semantico, il nome di marca deve corrispondere al prodotto perché questo si venda (cf. Vaxelaire 2005: 128); in questo senso, nel dare il nome a un prodotto "l'arbitrarietà è bandita" (Botton, Cegarra \& Ferrari 2002: 20). Tutto ciò deve inoltre rispondere a criteri di ordine fonetico: la lunghezza del nome - idealmente due-tre sillabe -, l'assenza di consonanti fricative, vibranti o nasali e di consonanti giustapposte (Botton, Cegarra \& Ferrari 2002: 66). È essenziale che il

10 Humbley (2006: 672) parla di "isonimia" a proposito dei nomi di marche e ragioni sociali che "funzionano" nelle principali lingue del mondo senza bisogno di traduzione. Nomi come Coca-Cola o Kodak fanno parte del vocabolario di gran parte dell'umanità su tutto il pianeta. 
nome "suoni bene" nelle principali lingue del mondo o perlomeno nelle lingue dei Paesi in cui il prodotto sarà commercializzato. Il nome si presenta infine come un compromesso, che massimizza i vantaggi e minimizza gli svantaggi in funzione di precisi obiettivi (Botton, Cegarra \& Ferrari 2002: 122).

Due sono i principali modelli che presiedono alla creazione di nuovi marchionimi. Collins (1977), tra i primi a studiare il fonosimbolismo in relazione ai nomi di marca (cf. Shrum \& Lowrey 2007: 48), battezzò Joyce Principle il principio secondo cui, nella creazione di marchionimi, si presuppone che (combinazioni di) suoni veicolino un significato, a prescindere dalla connotazione semantica della parola; il fonosimbolismo, appunto, ben presente nelle opere dell'autore irlandese (cf. Shrum \& Lowrey 2007: 40). Si tratta di una risorsa utile per coniare "meaningful brand names" senza basarsi sul significato del nome o di sue componenti (Shrum \& Lowrey 2007: 39). Vi si contrappone il Juliet Principle di shakespeariana memoria - "that which we call a rose, by any other name would smell as sweet" -: in questa prospettiva il nome è visto come mera etichetta, arbitraria e interscambiabile. Agli inizi le marche nascevano tendenzialmente secondo questo principio (si pensi al riuso di patronimici come nomi d'impresa o di prodotto), mentre il Joyce Principle è il più seguito nel marketing moderno.

\section{Linguistica: essere un nome. Aspetti teorici}

\subsection{Terminologia e definizioni}

Chi desidera indagare la natura linguistica dei nomi commerciali ${ }^{11}$ deve innanzitutto aprirsi un varco nella selva terminologica che li circonda.

11 Appunti di onomastica commerciale ricorrono in alcuni recenti manuali di onomastica: Ainiala, Saarelma \& Sjöblom (2012), incentrato sui nomi propri finlandesi, in cui il capitolo sui nomi commerciali conta ben 45 pagine rispetto alle sessanta dei toponimi e alle quasi ottanta degli antroponimi; Nübling, Fahlbusch \& Heuser (2012), dove i nomi di prodotto e d'impresa sono trattati nel lungo capitolo dedicato agli ergonimi. 
Nomi commerciali? o piuttosto nomi di marca, marchionimi, ma pure soprattutto in tradizioni di studi diverse da quella italofona - crematonimi, ergonimi, econimi? E qual è, se esiste, la differenza tra nomi di marca e nomi di prodotto?

Come segnalato da più parti (per es. in Ainiala, Saarelma \& Sjöblom 2012: 213; De Stefani 2005: 69; Platen 1997: 8n; Teutsch 2007: 51-52), manca una terminologia linguistica stabile e univoca per il ramo dell'onomastica che studia i nomi di entità appartenenti all'ambito commerciale; una confusione terminologica che è aggravata dal fattore interlinguistico e che in parte risale alle difficoltà nel delimitare chiaramente l'oggetto di studio (v. infra). Di seguito si propone una panoramica sulle principali proposte terminologiche e definitorie. ${ }^{12}$

Marca, più che un termine, è da considerare un concetto generico, che ha un equivalente in brand. ${ }^{13}$ Crematonimo (legato alla tradizione onomastica slava) ed ergonimo sono termini specifici, che fanno

Tra le istituzioni più recenti specificamente dedicate ai nomi (propri) in un contesto economico spicca il ciclo di convegni interdisciplinari Names in the Economy (cf. Bergien, Kremer \& Zilg 2008: 7), inaugurato ad Anversa nel 2006 e che, nella sua quinta edizione, si è svolto a Verona nell'estate 2015. I quattro volumi con gli atti dei convegni editi sinora (Anversa 2006: Kremer \& Ronneberger-Sibold 2007a; Vienna 2007: Wochele, Kuhn \& Stegu 2012; Amsterdam 2009: Boerrigter \& Nijboer 2012; Turku 2012: Sjöblom, Ainiala \& Hakala 2013) costituiscono un riferimento imprescindibile per chi svolga oggi una ricerca sui marchionimi.

12 L'elenco non è esaustivo; per altri termini cf. De Stefani (2005: 68-69) e, per il tedesco, Gabriel (2003: 25). Del resto, anche in questo settore dell'onomastica proliferano neoformazioni (perlopiù estemporanee), per es. Tyronyme riferito ai nomi di formaggi (Dufferain 2013). I termini in uso nell'ambito del marketing sono altri, troppo specifici per l'analisi linguistica qui proposta: Botton, Cegarra \& Ferrari (2002: 74-87) distinguono per es. tra "marca-prodotto" (Sottilette), "marca-ombrello" (Sony), "marca-gamma" (per una famiglia di prodotti: Mulino Bianco) e "marca-garanzia" o corporate brand (Walt Disney, Barilla).

13 Tanto l'etimologia di marca quanto quella di brand riportano alle funzioni primarie della marca: brand deriva dal norreno brenna 'bruciare', connesso alla marchiatura a fuoco del bestiame (cf. Lindemann 2010: 3); marca deriva dalla base germanica *mark- 'segnare', 'contrassegnare' (Caprini 1976: 75). Dal significato del protogermanico *marko 'limite', 'frontiera' si è sviluppato quello di 'segno (che contraddistingue un confine)', quindi 'traccia', 'impressione'. 
riferimento a classi più vaste dei soli nomi di prodotti: oggetti il primo, esercizi pubblici o creazioni dell'uomo l'altro, a seconda degli autori (cf. De Stefani 2004: 64-65). Inoltre alcuni studiosi, per es. Coletti (2010), utilizzano ergonimo anche per i nomi d'impresa o nomi aziendali (per i quali in italiano manca peraltro un termine tecnico di uso corrente; cf. De Stefani 2004: 64).

Ökonym 'econimo' è una proposta di Platen (1997) che rimanda alla funzione essenzialmente economica della marca (Teutsch 2007: 52). Il termine è stato ripreso in lavori successivi, soprattutto di area germanofona, ma non è particolarmente diffuso tra gli studiosi italofoni (e difficilmente riuscirà a imporsi; cf. De Stefani 2004: 64).

Il termine Warenname, che ha (avuto) largo corso negli studi di lingua tedesca, è problematico in quanto non include i servizi; non è chiara, poi, la differenza rispetto a Produktname. Manca, del resto, una versione adeguata in altre lingue, tra cui l'italiano. Inoltre la posizione di Koss (1996a: 1642), secondo il quale il termine si sarebbe oramai stabilito negli studi onomastici, è da considerarsi superata: oggi si osserva piuttosto la tendenza a usare Markenname o Produktname ed equivalenti.

Nome di prodotto sembra più preciso di nome di marca perché riferito a una categoria specifica. Ma per alcuni autori (v. De Stefani 2005: 69) si tratta di un iperonimo di nome di marca, che allude alla "designazione completa di un prodotto" (per es. Mifloc Purea di patate) e non solo agli elementi della stessa che hanno caratteristiche propriali (Mifloc).

Marchionimo è una proposta di Enzo Caffarelli (cf. De Stefani 2004: 64) che si pone sostanzialmente come sinonimo di nome di marca, alla stregua di antroponimo e nome di persona, e pare affermarsi sempre più in ambito scientifico, a prescindere dal gusto di un composto italo-grecizzante.

Nome commerciale (commercial name) è, a seconda degli autori, sinonimo di marchionimo (per es. in Coletti 2010) oppure iperonimo dello stesso, comprendente tutti i vari tipi di nomi che, dal punto di vista pragmatico, hanno a che fare con l'economia (Bergien, Kremer \& Zilg 2008: 10). Per Ainiala, Saarelma \& Sjöblom (2012: 210) “commercial 
names are names whose function is to direct the choices of consumers and investors and that have economic objectives in their use".

Sulla scorta di questa rapida rassegna, quali sono i termini funzionalmente più adatti al presente studio, tenuto conto della tradizione scientifica in cui esso si inserisce? La scelta si orienta ai criteri della massima univocità possibile, di una sufficiente genericità (la categoria, come si vedrà, non è chiaramente delimitabile) e della pertinenza agli studi linguistici. Quest'ultimo criterio invita ad accantonare espressioni come marca o brand, che non mettono l'accento sull'aspetto linguistico, ossia onomastico, del fenomeno. ${ }^{14}$ Gli altri permettono di escludere etichette troppo specifiche, ambigue o poco diffuse, come ergonimo, econimo o nome di prodotto. I termini plausibili che si è deciso di utilizzare in questa ricerca sono nome commerciale per 'nome di entità legata all'universo dell'economia' e, in rapporto di sostanziale sinonimia, marchionimo e nome di marca. ${ }^{15}$ Tali termini, si noti, non implicano una decisione in merito alla categoria grammaticale. Essi includono non solo i nomi che identificano un prodotto o un servizio, bensì anche i nomi d'impresa: sia quando sono utilizzati in quanto tali, sia quando sono usati per marchiare i loro prodotti, per es. Fendi nel costrutto una borsa Fendi (cf. Zardo 1996: 370). Non si distingue ulteriormente tra nomi di prodotto, di marca e d'impresa, concordando con Teutsch (2007: 53) che tale suddivisione è inadatta e superflua dal punto di vista giuridico ed economico, oltre che linguistico. Stabilire a priori che Barilla è, per es., un nome d'impresa non aggiunge nulla alla sua comprensione linguistica; senza dimenticare che uno stesso nome può manifestare diverse funzioni, in astratto e tanto più negli usi contestuali (De Stefani 2004: 64n; 2007: 246). Una distinzione terminologica precisa non può essere pertanto un presupposto dell'analisi sintattica; una volta delimitato l'ambito di pertinenza, come si fa con

14 Per contro, nella sua trattazione Teutsch (2007: 56) si decide proprio a favore del termine Marke, in quanto ritiene che il suffisso -name implichi appartenenza alla classe dei nomi propri (Teutsch 2007: 54), che egli non vuole presupporre per i nomi di marca.

15 Non si riprende, in quanto priva di fondamento, la distinzione di Riolo (2007: 7n) tra marchionimo 'nome brevettato o in corso di registrazione' e l'iperonimo nome commerciale, che prescinderebbe da un'eventuale registrazione legale. 
"nome commerciale" e "marchionimo", etichette più precise non servono, possono anzi pregiudicare l'analisi.

Come anticipato, non solo l'aspetto terminologico ma anche la definizione dell'oggetto di studio è, come osserva De Stefani (2008: 712-713), una delle questioni più discusse nella ricerca linguistica sui nomi commerciali. Manca tuttora una precisa definizione linguistica (cf. De Stefani 2004: 64); gli studi di questo settore, infatti, si richiamano sovente alle definizioni del marketing o del diritto (cf. Kremer \& Ronneberger-Sibold 2007b: 12), che però si basano su criteri e presupposti diversi.

Tra gli studiosi di onomastica si riscontra in generale una certa difficoltà ad allontanarsi da una visione referenziale. Si consideri per es. la definizione di Ronneberger-Sibold (2004: 557):

Warennamen sind Namen, unter denen Produkte und Dienstleistungen auf dem Markt angeboten werden. Als Namen unterscheiden sie sich von allen nicht-onymischen Bezeichnungen derselben Referenten, als spezielle Warennamen von deren eventuell parallel existierenden anderen Namen, die nicht dem Verkauf dienen.

La studiosa ritiene che il riferimento agli scambi economici (mercato, vendita) sia costitutivo dei nomi studiati, sì da preferire il termine Warenname a Produktname proprio in quanto allude all'utilizzo commerciale di tali nomi (Ronneberger-Sibold 2004: 558).

Un altro punto da considerare è l'estensione dell'oggetto di studio. Le definizioni correnti del nome di marca, in quanto basate su quelle giuridiche, tendenzialmente escludono i nomi d'impresa; uno sguardo ai dati linguistici mostra che in realtà non è sempre possibile né forse auspicabile - distinguere tra le due categorie quando si decide di analizzare gli usi discorsivi dei nomi legati al mondo del commercio.

Volendo definire il fenomeno in questione, che è linguistico ma pure inscindibilmente legato a una realtà extralinguistica - entro la categoria dei nomi (propri) ci si occupa di quelli legati al mondo del commercio, secondo la delimitazione proposta da Bergien, Kremer \& Zilg (2008) -, è inevitabile scontrarsi con difficoltà oggettive. Questo lavoro si fonda perciò su una concezione intuitiva (ma operativa) dei 
marchionimi, demandando ad altra sede il compito di delinearne una definizione linguistica precisa.

\subsection{Categoria grammaticale}

Il problema terminologico appena trattato si interseca con quello della categorizzazione grammaticale dei marchionimi, uno tra i principali aspetti teorici discussi negli studi linguistici sui marchionimi. La marca è un nome, sì, ma: proprio o comune?

Gli studiosi sono concordi nell'osservare che i nomi commerciali sono difficilmente classificabili dal punto di vista grammaticale; le loro proprietà rendono difficile una categorizzazione univoca (cf. Lötscher 2008: 25), siano esse strettamente linguistiche (semantiche, morfologiche, sintattiche) o referenziali (i marchionimi identificano non una singola entità, bensì una serie di prodotti identici: cf. Koss 1996a: 1642). La difficoltà dell'attribuzione categoriale è dovuta anche all'assenza di caratteristiche sperimentalmente affidabili per definire il nome proprio (La Fauci 2011: 201). Vi si aggiungono due premesse metodologiche problematiche: da un lato, la distinzione categoriale avviene perlopiù in astratto o sulla base di pochi esempi, eventualmente inseriti in frasi fittizie; dall'altro, i nomi commerciali sono una classe molto variegata al proprio interno. Gli usi metonimici ${ }^{16}$ (nome dell'impresa per il prodotto: comprare una Fiat) complicano ulteriormente il panorama.

I nomi d'impresa (corporate names o company names, Firmennamen) sono generalmente considerati nomi propri a tutti gli effetti, in particolare per ragioni pragmatico-referenziali: servono a identificare un individuo, una persona non fisica ma giuridica (cf. Bergien, Kremer \& Zilg 2008: 10; Koss 1996b: 1796; con restrizioni anche Van Langendonck 2007b: 32-33). Al polo opposto, considerati univocamente come nomi comuni - e la ricorrenza nei dizionari ne è quasi il sigillo -, stanno i marchionimi che hanno subíto deonimizzazione (cf. Ronneberger-Sibold

16 Con "metonimia", sulla scorta di Jakobson (1956), si intende un procedimento linguistico basato su una relazione di contiguità, in contrapposizione alla metafora, che si fonda invece su una relazione di similarità. 
2004: 558), ossia sono utilizzati anche per prodotti simili ma di marche diverse; l'elenco è lungo, da clacson a velcro (v. infra, §4.3).

La questione si complica per nomi di marca e di prodotto (la stessa terminologia, come accennato, non è univoca). Nella bibliografia si riconoscono le seguenti posizioni: tali nomi

1) si collocano in un territorio di transizione o al confine tra nomi propri e comuni; ${ }^{17}$

2) sono, a seconda dell'uso, nomi propri o nomi comuni;

3) sono nomi propri se nomi di marca (o d'impresa), nomi comuni se nomi di prodotto;

4) sono nomi propri. ${ }^{18}$

La prima ipotesi, che non prende posizione a favore di un'unica categoria grammaticale, si presenta in alcune variazioni sostanzialmente comparabili, che si sviluppano a partire da una considerazione: i nomi commerciali hanno proprietà morfosintattiche e semantiche tanto dei nomi propri quanto dei nomi comuni (cf. Molino 1982: 8). ${ }^{19}$ Migliorini (1927: 12) situa i nomi "di oggetti brevettati" nella "zona di confine fra i nomi proprî e gli appellativi”. Per Bergien, Kremer \& Zilg (2008: 10) i nomi di prodotto si collocano alle periferie dell'onimità. Koss (1996a: 1642) accenna a un territorio di transizione tra nomi propri e comuni, dal momento che i nomi di marca e di prodotto svolgono funzioni individualizzanti ma sono applicati a tutti i prodotti di uno stesso tipo (v. anche Šrámek 1996: 1564). Ancora, per Nübling, Fahlbusch \& Heuser (2012: 49) e Ronneberger-Sibold (2004: 558) i nomi di prodotto costituiscono una nuova categoria a sé stante, formata da classi di oggetti identici. La riproducibilità in serie dei prodotti, che comporta

17 Fortunatamente sembra passata di moda l'ansia di dare un nome a questo fenomeno, con risultati, più che ossimorici, del tutto illogici (per es. Gattungseigennamen: Harweg 1983).

18 Rari, e piuttosto datati, i contributi che sostengono la generale appartenenza dei nomi di marca alla categoria dei nomi comuni, eventualmente in statu nascendi (cf. Voigt 1989: 175).

19 Tale indeterminatezza è condivisa anche dalla neuropsicologia sulla base di ricerche i cui presupposti, da un punto di vista linguistico, sono discutibili. Gontijo \& Zhang (2007: 23) affermano: "brand names have a special neuropsychological status, but one that is different from proper names". 
un'infrazione al criterio della monoreferenzialità, fa propendere Platen (1997: 30-32) e Nübling, Fahlbusch \& Heuser (2012: 48-49) per una collocazione dei nomi di prodotto al confine tra proprio e comune, nonostante tali studiosi riconoscano loro un carattere onimico primario. Petit (2000; 2006), infine, imputa allo statuto "ibrido" dei marchionimi, situati all'intersezione degli ambiti giuridico e linguistico, l'indecidibilità categoriale. ${ }^{20}$

Come scrive Lötscher (2008: 36), questa posizione, non ulteriormente precisata, non è sostenibile, perché presuppone che vi siano limiti fluttuanti tra nomi propri e comuni o una gradualità nel passaggio dall'uno all'altro, senza che la differenza tra i due sia delineabile in maniera discreta. Criteri come 1" "atto di battesimo" alla base del nome proprio, o meglio il "riferimento al codice", segno della natura metalinguistica del nome proprio (cf. Jakobson 1957: 131 e, su questo, La Fauci 2011: 202), sono invece specifici dei nomi propri rispetto a quelli comuni.

Nella seconda prospettiva, la distinzione categoriale avviene sulla base del contesto enunciativo (cf. Gabriel 2003: 38): i marchionimi non sono degli ibridi ma sono, a seconda degli usi, nomi propri o nomi comuni. Per Berger (1976: 376-377) i nomi di prodotto, che sono nomi propri in quanto risalgono a un atto di denominazione, sono utilizzabili anche come nomi comuni, per es. se preceduti da un determinatore. Per Van Langendonck (2007a: 235; 2007b: 32) i nomi commerciali sono "proprio-appellative lemmas", ossia entrate del dizionario che possono essere usate tanto come nomi propri quanto come nomi comuni.

Van Langendonck (2007a: 236) correla l'attribuzione categoriale a una necessaria differenziazione su base referenziale. Lo studioso distingue tra nomi di marca e d'impresa, che tipicamente sono nomi propri, e nomi di prodotto, che normalmente sono nomi comuni (Van Langendonck 2007b: 23). Così la sua visione sfocia nel terzo tipo di concezione, della quale un altro autorevole esponente è Vaxelaire (2005). Insieme ad altri autori, egli distingue entro la classe dei nomi commerciali due gruppi: i nomi di marca, assimilabili ai nomi propri, e

20 Cf. Petit (2000: 167): "Le N[om]D[éposé] est [...] une entité dont le statut sémiotique est ambivalent, hybride, tenant à la fois du nom propre et du nom commun sans avoir pleinement les propriétés de l'un ou l'autre". 
i nomi di prodotto, che si comportano come nomi comuni (cf. Vaxelaire 2005: 345-346 e, inoltre, Šrámek 1996: 1564, Fèvre-Pernet \& Roché 2005 e Laurent 2007: 37; Jonasson 1994: 29, invece, considera nomi propri quelli che designano un'impresa). ${ }^{21}$ Si tratta di una distinzione aprioristica, elaborata a volte su base referenziale (Petit 2006: 692) o addirittura partendo dagli imballaggi dei prodotti (Fèvre-Pernet \& Roché 2005): "Une fois établie la distinction entre noms de marques et noms de produits, il est plus facile de considérer a priori que les premiers fonctionnent en tant que noms propres et que les seconds sont des noms communs" (Vaxelaire 2005: 349).

Infine, vi sono autori che sostengono l'appartenenza dei nomi di prodotto ai nomi propri. Per Lötscher (2008: 26 e 31-32), che argomenta sulla base del contenuto (Inhalt) dei nomi, ${ }^{22}$ i nomi propri rispettano le tre condizioni seguenti: assenza di contenuto intensionale; unicità di riferimento; denotazione derivante da un atto denominativo. Siccome i nomi di prodotto possiedono queste caratteristiche, essi vanno considerati come nomi propri a tutti gli effetti, pur non disconoscendo la possibilità di utilizzarli anche come nomi comuni (Lötscher 2008: 38).

La visione di Lötscher, supportata da un'argomentazione rigorosa, appare più plausibile rispetto al punto di vista proposto da Vaxelaire, Van Langendonck e altri, per i quali è possibile o addirittura auspicabile distinguere tra varie categorie di nomi commerciali in ragione di aspetti extralinguistici. In primo luogo, tali discussioni sono spesso condotte (per es. nei due autori citati) sulla base di esempi fittizi, se non astraendo del tutto dal contesto linguistico. La concezione di Lötscher, al contrario, presenta il vantaggio di fondarsi non sui referenti concreti né su frasi formate ad hoc ma su principî relativamente consolidati nel panorama degli studi onomastici. Inoltre una visione unitaria dei nomi commerciali ben si attaglia a uno studio come il presente, che si pone di fronte al proprio oggetto non con un atteggiamento classificatorio, bensì

21 Una concezione paragonabile anche in Hansack (2004: 63), più confuso e discutibile. Per Petit (2006: 692-693) il funzionamento dei nomi di prodotto li rende accomunabili tanto al nome proprio (referenza metalinguistica) quanto al nome comune (classe di occorrenze del referente sul mercato).

22 Si tratta di caratteristiche valide a livello interlinguistico; differenze grammaticali tra nomi propri e comuni, nota lo studioso, variano invece a seconda delle lingue e quindi non sono da lui considerate (Lötscher 2008: 26). 
con l'attitudine di osservare e descrivere i dati senza pregiudizi tassonomici o categoriali. Una visione che mette al centro - come e ancor più di quanto fa Lötscher - la concezione dei nomi propri, jakobsonianamente intesi, come entità dal valore spiccatamente metalinguistico ci sembra sufficiente per affrontare l'analisi.

\subsection{Evoluzioni nell'uso linguistico quotidiano}

La capacità dei marchionimi di travalicare i confini categoriali emerge con particolare evidenza nel passaggio da denominazioni distintive a designazioni generiche per tutti i prodotti di un certo tipo, indipendentemente dalla ditta produttrice (cf. Teutsch 2007: 107): scotch per ogni sorta di nastro adesivo, $k$-way per 'giacca a vento leggera' e numerosi altri esempi, dei quali non di rado gli stessi utenti della lingua sono consapevoli.

Si parla, in questi casi, di "genericizzazione", "genericidio", "degenerazione", "volgarizzazione" (Zardo 1996: 369) o "lessicalizzazione" (Botton, Cegarra \& Ferrari 2002: 165). Il processo di allargamento semantico, per cui un nome proprio acquista carattere descrittivo, può sfociare in un processo deonomastico, nel passaggio della marca da nome proprio a nome comune. Tale passaggio è generalmente consacrato dalla registrazione del nome nei dizionari, magari con iniziale minuscola: è "[i] 1 ritorno del nome all'anonimato" (Botton, Cegarra \& Ferrari 2002: 31).

La valutazione negativa implicata da espressioni quali "degenerazione" o "genericidio", che spinge alcuni a preferire il termine neutro "genericizzazione" (Vande Casteele 2014: 655), è un riflesso della prospettiva del marketing, per il quale tale processo rappresenta non "una consacrazione lusinghiera", bensì una perdita di valore e, eventualmente, di protezione giuridica (Botton, Cegarra \& Ferrari 2002: 165). ${ }^{23}$ Come scrive Petit (2006: 700), il nome comune è l'asintoto del nome di marca: "ce dont il peut s'approcher, mais qu'il ne doit jamais atteindre".

23 Soltanto Minestroni (2010: 139) pare ignorare i risvolti problematici del fenomeno: "si tratta di un fenomeno assolutamente positivo e prestigioso per le aziende [...]. Scompare il mascara, sostituito dal Rimmel. Un grande vantaggio per il produttore". 
Le marche possono diventare vittima del proprio successo (Masson 2000); il passaggio a nome comune, se da una parte ne rappresenta la consacrazione, dall'altra comporta la perdita dello specifico carattere (onimico) di marca, dal momento che vengono a mancare l'identificazione di un prodotto unico, la divergenza intensionale tra contrassegno e referente e l'attribuzione attraverso un atto di battesimo (Teutsch 2007: 106). Infatti chiunque può utilizzare un nome di marca registrato, ma l'uso metalinguistico è riservato alla sola ditta produttrice, ${ }^{24}$ altrimenti sfocia in un abuso (dal punto di vista giuridico).

Gli usi dei marchionimi nella lingua comune non sono tutti ugualmente problematici per il produttore. Lötscher (1995: 455-456) distingue gli usi metonimici del produttore per il prodotto, che possono essere occasionali (guidare una Fiat) o lessicalizzati (in ingl. hoover per 'aspirapolvere'), e il fenomeno per cui proprietà connotative del referente diventano parte del significato intensionale di un segno che denota un referente unico, come nei casi di Föhn, Tempo o Knirps. Questo mutamento di significato apre la strada alla genericizzazione e alla conseguente lessicalizzazione del marchionimo: se Föhn non è più solo un "nome-etichetta", ma implica le proprietà semantiche di 'asciugacapelli', allora è possibile che tutti gli asciugacapelli siano ribattezzati föhn (e l'iniziale minuscola, con l'ovvia eccezione del tedesco, è spesso convenzionale traccia grafica di questa reinterpretazione). Il primo uso metonimico citato, per contro, non risulta particolarmente problematico per una ditta: difficilmente una Fiat diventerà sinonimo di 'automobile'.

Petit (2006: 700) distingue invece tra "banalizzazione", ossia utilizzo di un marchionimo al posto di un lessema esistente (frigidaire per réfrigérateur), ${ }^{25} \mathrm{e}$ "lessicalizzazione", quando il marchionimo colma una lacuna lessicale (nylon, abribus).

24 Su questo cf. Petit (2006: 699-700): "Du point de vue juridique, le Coca-cola [sic] est un soda qui s'appelle Coca-Cola et rien d'autre, un Frigidaire un réfrigérateur qui s'appelle Frigidaire, etc. [...] le nom n'a pas pour objet - juridiquement - d'encoder des propriétés ou de résumer un prédicat définitoire (Kleiber 1984) mais de distinguer des entités par le simple fait qu'elles portent des noms différents".

25 Altrove ne parla nei termini di un "comblement diaphasique pour tous les $\mathrm{N}$ [oms]D[éposés], qui fournissent une dénomination tendanciellement familière", opposto al "comblement d'une lacune" (Petit 2000: 188). 
Il primo stadio verso la genericizzazione è rappresentato, per Botton, Cegarra \& Ferrari (2002: 166), dalla "personalizzazione", quando il marchionimo è usato non come "modificatore propriale" (Vande Casteele 2014: 657) ma come nome: non l'estratto Liebig, bensì il Liebig, in un uso definito ellittico e metonimico (cf. Botton, Cegarra \& Ferrari 2002: 30). Il secondo stadio è la "banalizzazione" (non come la intende Petit), in cui il nome di marca è usato per un'intera categoria di prodotti (liebig per tutti gli estratti di carne): si potrebbe definirlo nei termini di un'antonomasia (vossianica), per cui un nome proprio è applicato a entità diverse da quella originaria, che ne condividono delle qualità (parla di antonomasia per es. Zardo 1996: 369). Segue, infine, la "lessicalizzazione" (anche qui, intesa diversamente da Petit): il marchionimo dall'uso allargato entra nel dizionario. ${ }^{26} \mathrm{~A}$ questo punto gli è riconosciuto un significato in senso stretto.

Dati i rischi giuridici ed economici che possono derivare alla ditta dall'abuso di marchionimi, essa protegge i nomi in suo possesso regolamentandone l'uso linguistico. L'International Trademark Association (INTA 2012) riassume le direttive per l'uso proprio della marca nell'acronimo ACID (Adjective, Consistent, Identification or Status, Distinctive): le marche vanno usate come aggettivi ${ }^{27}$ e al singolare; le raffigurazioni della marca devono essere sempre coerenti nei vari testi; è necessaria un'identificazione come ${ }^{\circledR} \mathrm{o}$ trademark; le marche devono distinguersi graficamente rispetto al testo circostante. Il primo punto è il più interessante dal profilo linguistico. L'INTA distingue, al proposito, fra trademarks e service marks (Kleenex, FedEx) da una parte, che sono "proper adjectives", e trade names dall'altra (come Reebok), che sono nomi propri; i primi dovrebbero essere usati "as an adjective qualifying a generic noun that defines the product or service" (INTA 2012). Può accadere, in un tentativo che ha del disperato, che la ditta si rivolga ai consumatori chiedendo un uso corretto, ossia aggettivale, dei nomi di marca. È il caso delle campagne pubblicitarie di Xerox volte a scongiurare l'uso generico della marca, nominale e verbale (cf. Platen 1997: 122n).

26 Non si tratta, peraltro, di un'entrata “definitiva" nel dizionario, come ritengono gli autori; basta uno sguardo alle diverse edizioni di un'opera lessicografica per sincerarsene. Sull'aspetto lessicografico cf. Riolo (2007) e Zardo (1996) e, più avanti, il $§ 4.2$.

27 O, si potrebbe dire meglio, come modificatori; cf. Vande Casteele (2014: 657). 
Nonostante gli sforzi profusi dall'azienda, peraltro, (to) xerox si è imposto in inglese con il significato di 'fotocopiare' e 'fotocopia'.

L'uso nominale rappresenta il primo passo verso la genericizzazione e come tale va evitato (cf. Botton, Cegarra \& Ferrari 2002: 167; Solly 2002: 228). Al contrario, l'uso "proprio" del nome di marca (proper use: Solly 2002: 211) coincide con l'uso "come nome proprio", nella misura in cui ai nomi propri si può riconoscere carattere aggettivale o di modificatore (su questo si tornerà).

D'altro canto, non in tutti i casi l'uso metonimico di un marchionimo (nome dell'impresa per un suo prodotto) porta alla genericizzazione. Difficilmente, si è osservato, una Fiat passerà a concetto generico per indicare l'automobile. In generale i nomi di prodotti molto diffusi, innovativi e/o che sono leader di mercato in un determinato settore merceologico (cf. Minestroni 2010: 144) sono più suscettibili di seguire la trafila fino alla lessicalizzazione rispetto ai nomi d'impresa. Ciò avviene perché, da un lato, con i prodotti innovativi si introduce sul mercato un oggetto prima inesistente, che pertanto non ha (ancora) una designazione generica; dall'altro, i marchionimi si accostano ai sinonimi generici, e talvolta si impongono su di essi, in quanto nomi particolarmente memorabili ed efficaci nella loro brevità (cf. Botton, Cegarra \& Ferrari 2002: 249; Platen 1997: 124-125). ${ }^{28}$ Ma una motivazione più profonda per quest'uso dei marchionimi va ricercata forse altrove: come per le antonomasie vossianiche (un cerbero, un catone), che sfruttano la carica evocativa del nome proprio facendo appello a un codice culturale comune, così anche gli usi generici della marca rappresentano la consacrazione implicita di un sapere che il parlante ritiene condiviso con l'interlocutore, "il che è di per se stesso fonte di piacere specifico" (Botton, Cegarra \& Ferrari 2002: 29).

Così si potrebbe rispondere a Gutschmidt (1982: 21), che lamenta la mancanza di una classificazione dei marchionimi basata sulla possibilità di passare ad appellativi e nota: "so verwandeln sich Namen von Chemiefasern relativ schnell in Appellativa (vgl. Wolpryla), während das bei Zigarettennamen nicht der Fall sein dürfte". I primi sono in genere nomi di prodotti innovativi, i secondi mere etichette applicate a oggetti (e nomi comuni) preesistenti. 


\section{Linguistica: studi descrittivi}

La panoramica sulla bibliografia nel settore dei marchionimi si chiude con la presentazione di alcuni filoni di ricerca ricorrenti negli studi linguistici, d'impianto descrittivo più che teorico. ${ }^{29}$

\subsection{Analisi formale}

L'accostamento formale ai marchionimi riguarda soprattutto aspetti morfologici e studia di quali elementi linguistici è composto il nome. I tentativi di elaborare tipologie e classificazioni su base formale costituiscono larga parte delle analisi onomastiche dei nomi commerciali (Platen 1997: 5; cf. inoltre Ainiala, Saarelma \& Sjöblom 2012: 217).

Tali studi si basano di solito su insiemi di dati relativamente omogenei: marchionimi appartenenti a uno specifico settore commerciale (per es. farmaci: Anreiter 2002; prodotti alimentari: Muselmann 2010, Zilg 2006; giocattoli: Fèvre-Pernet 2007; casalinghi e prodotti di elettronica: Gabriel 2003) e/o relativi a una data lingua o nazione (cf. Vincze 2008 sui nomi di prodotto ungheresi e alcuni dei saggi contenuti in Boerrigter \& Nijboer 2012).

I principali punti di vista adottati nella descrizione di nomi commerciali sono due: da un lato si studiano le modalità di formazione dei nomi (dalla prospettiva, quindi, del creatore di nomi); dall'altro, in parte in maniera correlata, si osservano le connotazioni o associazioni che il nome veicola (nell'ottica del consumatore, destinatario fondamentale - per le ragioni commerciali che si immaginano - del nome).

Quanto alla formazione dei marchionimi, va sottolineato innanzitutto che essi sono "artefatti sincronici" (Kalverkämper 1978: 302) e, benché non di rado formati sulla base di elementi linguistici preesistenti, si situano al di fuori dell'evoluzione diacronica attraverso il particolare

29 Una panoramica recente (e corposa) sugli studi linguistici dedicati ai marchionimi, in particolare negli ultimi quarant'anni, è contenuta in Eckkrammer \& Thaler (2013b: 17-53). Si vedano inoltre Bergien, Kremer \& Zilg (2008: 7-10); Koss (1996a: 1642); Platen (1997: 2-5); Ronneberger-Sibold (2004: 567-568 e 602-603). 
atto di creazione che ne è all'origine. ${ }^{30}$ Non sono nomi con un'etimologia plurisecolare ma creazioni consapevoli e deliberate, immesse nel mondo linguistico attraverso la pubblicità, la comunicazione d'impresa, i prodotti stessi. ${ }^{31}$ In relazione ai marchionimi non si può pertanto parlare di "etimologia" in senso stretto, bensì piuttosto di formazione delle parole (cf. Thornton 2004) o di "tipi denominativi" (Serianni 1978: 56).

Cotticelli Kurras (2007; 2008) e Ronneberger-Sibold (2007; 2008a), che si sono occupate rispettivamente di marchionimi italiani e tedeschi nell'ambito di un'indagine contrastiva, distinguono tra diversi tipi morfologici: 1) ripresa di parole o sintagmi esistenti (Fulmine); 2) neoformazioni: lessemi regolari e irregolari (Enochina; Supertab); 3) neoformazioni: sintagmi regolari e irregolari (Acqua vesuviana; Asti spumante). I neologismi si distinguono ulteriormente sulla base delle lingue coinvolte (italiano: Campomilla; ibridi: Nicotussina; non italiano: Oftalgon) e della tipologia di creazione: composti (Nannasciutto), affissazioni (Bismutol), incroci o "parole-macedonia" (Aperolitivo), acronimi e abbreviazioni $(F D B)$ e così via (cf. Cotticelli Kurras 2008: 696; per una classificazione simile cf. Ronneberger-Sibold 2004: 583-584).

Lo studio morfologico dei marchionimi si interseca con quello, latamente psicolinguistico, delle associazioni. Giuridicamente, lo si è visto nel $\S 2.1$, il nome di marca non può avere un significato che sia descrittivo del prodotto designato, ma d'altra parte è necessario che esso veicoli un significato che abbia connotazioni positive e, in qualche modo, descriva il prodotto: i marchionimi devono significare. ${ }^{32}$ Cotticelli Kurras $(2011 ; 2014)$,

30 Anche se, com'è ovvio, una volta immessi nella lingua - e una volta che i parlanti se ne sono appropriati, sottraendoli ai dominî esclusivi e normativizzati di marketing e diritto -, pure i marchionimi sono passibili di mutamenti diacronici a più livelli, dalla fonetica alla morfologia alla semantica (sulla quale cf. §3.3). Questa è la differenza principale dei marchionimi rispetto alle categorie tradizionali dei nomi propri, benché spesso ignorata dagli studiosi che paragonano i vari tipi (per es. Ainiala, Saarelma \& Sjöblom 2012: 215-216): i primi sono frutto di una creazione, i secondi il risultato di una Entstehung, di un divenire.

Petit (2006: 696) osserva al proposito una fondamentale differenza rispetto ai nomi propri prototipici: “le nom propre n'est pas contraint, sur quelque plan que ce soit, de signifier. S'il se charge d'une valeur, c'est uniquement par un accident de l'histoire. En revanche, le N[om]D[éposé] se doit de signifier, ne serait-ce que pour fidéliser la clientèle et l'élargir. Cette obligation sémantique est inscrite dans sa sémiotique, tout comme elle l'est dans celle du nom commun". 
Ronneberger-Sibold (2008b) e Ronneberger-Sibold \& Wahl (2014) classificano le associazioni veicolate dai marchionimi secondo alcuni ambiti di appartenenza: potere, tradizione, status, aggressività, romanticismo ecc.

Sorgono dei problemi quando la classificazione dei marchionimi avviene non tanto sulla base della struttura morfologica o delle associazioni (che sono pur sempre un'interpretazione, ma basata su aspetti semantici e luoghi comuni largamente condivisi), bensì in relazione alle motivazioni di chi ha dato il nome. In particolare per i nomi d'impresa ricorrono nella letteratura affermazioni discutibili, unite a una limitata consapevolezza del problema da parte degli studiosi (fa eccezione Serianni 1978: 56): su quale base si attribuisce Marco Polo alla categoria "scienze, economia, religione", mentre Jeanne d'Arc rientra nell'"ambito politico e militare", come sostiene Kuhn (2008: 644 e 646)? Ancor più problematiche le classificazioni miste, come quella che riporta Laurent (2007: 38-39), in cui a classi formali (patronimici, neologismi) si accostano classi interpretative (nomi simbolici).

\subsection{Marchionimi nelle opere lessicografiche}

In ragione della loro natura linguistica non univocamente definita (cf. §3.1 e 3.2), non stupisce che i marchionimi costituiscano un caso sempre più imbarazzante (Rey-Debove 1995: 119) per i lessicografi, che devono rendere ragione tanto di uno statuto, nell'uso linguistico, simile a quello del nome comune, quanto del carattere propriale giuridicamente implicito nei nomi di marca (cf. Petit 2006: 702).

Studi di taglio metalessicografico evidenziano "un'attitudine lessicografica incerta verso i marchionimi" (Zardo 2007: 267) e il carattere perlopiù arbitrario delle scelte di chi compila i dizionari (Riolo 2007: 60 ), nei quali i criteri di inclusione o di esclusione, anche relativamente ai nomi di marca, non sono praticamente mai esplicitati. ${ }^{33}$ Il confronto tra opere diverse, ma anche tra diverse edizioni dello stesso dizionario

33 Teutsch (2008) si occupa invece della questione da un punto di vista giuridico e generale: per quali motivi i nomi di marchio registrato sono indicati come tali nelle opere di consultazione. La legislazione relativa pondera gli interessi di chi 
(cf. Riolo 2007: 52-56 per dizionari editi da Garzanti; Zardo 2007: 256-265 per due edizioni dello Zanichelli e una del dizionario a cura di De Mauro), illustra notevoli divergenze, sia per quanto riguarda $\mathrm{i}$ marchionimi inclusi o esclusi, sia per il trattamento grafico (maiuscola iniziale) e giuridico (la segnalazione di ${ }^{\circledR} \mathrm{o}$ marchio registrato). Più che di "lacune e imprecisioni di vario genere" (Riolo 2007: 19), che presupporrebbero la fattibilità di un trattamento coerente, la situazione lessicografica riflette un panorama composito e lungi dall'essere chiarito da un punto di vista linguistico. Si registra inoltre una forte variabilità sull'asse diacronico: "L'attestazione di un marchio nel vocabolario è tanto rapida quanto spesso, per nemesi, effimera o incerta" (Zardo 2007: 253).

Ecco alcuni esempi di divergenze. Bostik è segnalato nel dizionario a cura di Tullio De Mauro (2006), ma non è presente nello Zingarelli dello stesso anno (Zardo 2007: 257), mentre Kamut c'è nello Zingarelli ma non nel De Mauro (Zardo 2007: 259). In due diverse edizioni, lo stesso dizionario (per es. Zingarelli 1993 e 2006) può riportare o non riportare uno stesso nome di marca (Lacoste c'è solo nel più vecchio, Memotel solo nel più nuovo; Zardo 2007: 260), con iniziale maiuscola o no (teflon nel 1993 e Teflon nel 2006; Zardo 2007: 263), come marchio registrato o no (nylon perde ${ }^{\circledR}$, mèta l'acquista; Zardo 2007: 260-261).

Quando un nome di marca presente nel dizionario scompare in un'edizione successiva, pare opportuno parlare, più che di "morte" dei marchionimi (cf. Zardo 2007: 253), di uscita dal luogo comune: dal momento che, per i nomi propri, l'acquisizione di un significato connotativo, condizione necessaria per l'inclusione nel dizionario, è legata a ragioni di natura sociale (conoscenze condivise all'interno di una comunità), le quali a loro volta stanno in funzione del (frequente) ricorrere di un oggetto linguistico nei discorsi, è comprensibile che la progressiva decadenza di un prodotto, per es. a causa di tecnologie divenute obsolete, comporti pure la scomparsa dello stesso dai discorsi linguistici e, infine, dai dizionari.

Quale criterio principale per l'inclusione di marchionimi nei dizionari, Riolo (2007: 49) adduce la diffusione e la notorietà degli

possiede il monopolio dei nomi e la necessità di nuovo materiale linguistico per l'uso quotidiano. 
stessi, ${ }^{34}$ parlando di "termini importanti che il lettore comune si aspetta di trovare registrati". Si tratta peraltro di criteri più ideali che reali e fortemente soggettivi; Riolo registra tra le assenze anche duralluminio e safranina, rolex ma non swatch (Riolo 2007: 49). Inoltre il criterio della diffusione non è sufficiente: vi sono marchionimi che ricorrono di frequente nel parlato e nello scritto senza per questo essere registrati nei dizionari. Si pensa in particolare agli usi metonimici del nome d'azienda per un suo prodotto specifico (le Marlboro, la Lacoste, le Clark's), ${ }^{35}$ nei quali pure è riconoscibile un significato connotativo oltreché denotativo (si parla di sigarette, maglietta polo, scarpe). Tali usi "eponimi" non ricorrono nei dizionari non perché siano troppo numerosi per essere registrati tutti, come ritiene Rey-Debove (1995: 119), bensì, da un lato, in ragione della loro natura combinatoria (sono, per così dire, intrinsecamente determinati, ossia possono prescindere da un articolo solo in contesti specifici; cf. Parte III, §1.1.3) e, dall'altro, perché permane un valore metalinguistico: la Lacoste è una polo di marca Lacoste, ossia è partecipe, a qualche livello, di un processo di battesimo per cui qualcosa è "chiamato Lacoste". Inoltre il valore antonomastico del marchionimo resta in certo modo subordinato al valore di "azienda" (cf. Zardo 1996: 377).

In generale, come osserva Zardo (1996: 372), marchionimi relativi a un prodotto o servizio specifico, registrati (e a volte anche brevettati), sembrano più passibili di un uso generico, pur continuando a riferirsi, per ragioni giuridiche, al referente originario. Marchi antonomastici ${ }^{36}$ "volgarizzati", quali walkman o nylon, sono i più attestati

34 Si veda a tal proposito già Migliorini (1946: 18): "Alcune volte il lessicografo dovrà registrare anche quelle parole «private» che sono i nomi brevettati: termini come aspirina, grammofono, razzia, varecchina, che non si può fare a meno di inserire in un vocabolario perché divulgatissimi, sono rivendicati come proprietà esclusiva da altrettante ditte".

35 Petit (2006: 694) osserva in questi casi una relazione d'iponimia: le automobili Peugeot in rapporto alle automobili in generale. L'unica proprietà delle vetture di questo sottotipo è di essere prodotte da Peugeot.

36 A volte è il dizionario stesso a segnalarli come tali. Cf. DISC s.v. scotch: "Denominazione commerciale, che costituisce marchio registrato, di un tipo di nastro adesivo; per antonomasia, nastro adesivo" (corsivo aggiunto). 
nei dizionari (Zardo 1996: 372) ${ }^{37} \mathrm{Si}$ nota che per questi nomi un uso attributivo è difficilmente pensabile: non è un caso. Marchionimi che nascono più come nomi che come aggettivi sono più propensi a volgarizzarsi, anche per ragioni extralinguistiche: nascono insieme all'oggetto che denominano, spesso innovativo, non come etichetta apposta a un prodotto appartenente a una serie preesistente (cf. supra, §3.3).

Al contrario, gli usi metonimici (nome dell'impresa associato a un suo prodotto particolare), per es. Ferrari o Nikon, che Zardo (1996: 372) definisce anch'essi antonomastici in quanto designano il prodotto per antonomasia dell'azienda, ${ }^{38}$ sono usi nominali in relazione paradigmatica con usi predicativi ( $i$ Lego corrisponde a $i$ cubetti Lego). Capita che lo stesso marchionimo si possa inoltre usare come predicazione di un nome comune indicante un altro tipo di prodotto: la tuta Adidas rispetto a le (scarpe) Adidas. In genere questi nomi di marca non sono registrati nei dizionari, come non lo sono quei marchionimi non associati in maniera univoca a un prodotto specifico della ditta e usati perlopiù attributivamente: solo in contesti particolari un Toshiba potrebbe sostituire un videoregistratore Toshiba (Zardo 1996: 372-373). In prospettiva linguistica, dunque, è possibile estrapolare ragioni combinatorie alla base dei tipi di marchionimi che (non) ricorrono nelle opere lessicografiche.

\subsection{Analisi contestuale}

Sono rari, non solo per l'italiano, gli studi di linguistica che si occupano dei marchionimi non in quanto voci di un elenco o lemmi di un dizionario, bensì come parte della lingua in uso (cf. Vande Casteele 2012: 94), come elementi studiati in funzione delle relazioni che instaurano con il contesto linguistico. Un'analisi morfosintattica, quindi, non nel

37 Sarebbe interessante un confronto con edizioni più recenti dello Zingarelli, per es. quella del 2015, dove ricorrono tra gli altri anche iPhone, iPod, iPad, Bluetooth e TomTom, non (così) volgarizzati.

38 Cf. Zardo (1996: 370): "poco importa [...] che la Nikon produca oggi anche occhiali, quando è normale identificare questo marchio, salvo contesti specifici, con un apparecchio fotografico". 
senso in cui è intesa negli studi onomastici, ossia come analisi di nomi commerciali composti di più elementi e delle relazioni tra essi (cf. Ronneberger-Sibold 2004: 599; De Stefani 2005: 75-77).

Analisi di tipo contestuale sono state avanzate in base a dati sia scritti, sia orali (v. De Stefani 2005, 2007, 2008, 2009 per l'uso di marchionimi nell'interazione), distinguendosi in ciò da analisi fondate su liste di nomi o su esempi inventati. Al fine di descrivere l'effettivo funzionamento linguistico dei marchionimi, che presentano un'ampia varietà di usi morfosintattici (cf. De Stefani 2007: 246), basarsi su esempi fittizi è problematico, in quanto i primi (e spesso i soli) usi che vengono in mente sono quelli "classici": nome d'impresa usato referenzialmente, nome d'impresa per il suo prodotto, nome di prodotto. Come si vedrà nella parte analitica, non si tratta che di una minima parte delle combinazioni effettivamente ricorrenti nei testi, benché i token attribuibili a questi tipi siano in effetti i più numerosi.

Vande Casteele (2012) approfondisce i vari tipi di costrutti in cui appaiono nomi d'impresa in un corpus di testi giornalistici spagnoli e le informazioni semantiche che essi veicolano. I nomi d'impresa ricorrono spesso accompagnati da elementi descrittivi che non sono necessari per stabilire il riferimento ma offrono al lettore (o, meglio, ribadiscono) "background information", come in "La productora alemana de automóviles deportivos Porsche" (Vande Casteele 2012: 94). La studiosa illustra come i diversi costrutti si distribuiscano in parti distinte dell'articolo di giornale: nel titolo il riferimento all'impresa avviene perlopiù attraverso il solo nome proprio ("TeliaSonera rechaza la oferta 'amistosa' de France Télécom"); nel sottotitolo ricorre spesso un sintagma nominale descrittivo ("La firma escandinava considera la oferta insuficiente"), mentre l'incipit dell'articolo riporta una costruzione appositiva contenente il nome d'impresa ("El operador sueco-finlandés TeliaSonera ha rechazado la oferta de fusión 'amistosa' lanzada por France Télécom"; Vande Casteele 2012: 101).

Anche Zardo (1997) si occupa di stampa periodica, stavolta italiana, analizzando le ricorrenze di determinatori con i nomi d'impresa: in alcune riviste specializzate egli osserva "una forte tendenza a omettere l'articolo davanti a marchi indicanti un'impresa" (Zardo 1997: 28), mentre per altre registra, con lo stesso tipo di nomi, una presenza costante dello stesso (su questa variazione cf. Parte III, §1.1.2). Pure 
gli usi antonomastici del nome d'impresa per un suo prodotto sono preceduti da articolo (Zardo 1997: 28). Lo studioso rileva inoltre alcune "sequenze in cui il marchio svolge una funzione aggettivale/appositiva": "il gruppo Fiat", "il caso Volkswagen" e simili (Zardo 1997: 30). Ciò avviene anche nel linguaggio pubblicitario, in cui, anzi, l'uso di nomi comuni descrittivi accanto al marchio di fabbrica è d'obbligo per scongiurare la genericizzazione dello stesso (v. §4.3): si hanno pertanto casi come "Il climatizzatore Toshiba", "La carta American Express" (ma anche "Il Pinguino DeLonghi"), oltre a sequenze del tipo "lo spirito Qantas" o "lo stile Rover" che - ipotizza lo studioso - proprio l'uso pubblicitario ha contribuito a diffondere (Zardo 1997: 38).

In un'analisi relativa a materiale in tedesco, sia scritto (articoli di giornale) che parlato (videoregistrazioni di interazioni tra clienti in un negozio), De Stefani (2007) approfondisce le ricorrenze di marchionimi nella prospettiva dell'analisi conversazionale. La domanda alla base dell'analisi non è tanto "che cos'è un nome commerciale", quanto piuttosto come viene utilizzato in determinati contesti (De Stefani 2007: 234), laddove il come si riferisce non solo ad aspetti strettamente morfosintattici, bensì include anche riflessioni sulla funzione svolta dal nome nella strutturazione di un discorso scritto o in uno scambio interazionale. Uno stesso nome commerciale, in questo caso il marchio svizzero di abbigliamento Tarzan, non presenta solo usi onimici ma può essere utilizzato per categorizzare referenti diversi (De Stefani 2007: 246). Dal punto di vista morfosintattico, Tarzan ricorre sia come nome isolato (con funzioni di soggetto e oggetto), sia in composti, perlopiù come modificatore (De Stefani 2007: 237-238): esso identifica, di volta in volta, prodotti della marca ("Tarzan-Shirts", "Tarzan-Gürtel”), i fondatori dell'azienda ("Tarzan-Köpfe", "Tarzan-Männer") o altro ancora (“Tarzan-Fans", “«Tarzan»-Philosophie”).

Lo studio di conversazioni tra clienti al supermercato, nell'ottica di un"“onomastica interazionale" (De Stefani 2008: 726), illumina un aspetto cruciale relativo all'impiego di nomi commerciali nel parlato, un settore sin qui ignorato dagli studi sui marchionimi. I parlanti inseriscono i marchionimi nella conversazione costituendoli e negoziandoli come oggetti di discorso il cui referente può cambiare durante l'interazione (De Stefani 2008: 726). Avviene così che, in un dialogo, un 
partecipante usi "gillette" non tanto come "riferimento onimico" a un marchionimo ma alludendo genericamente alle lamette da barba (De Stefani 2008: 720). In seguito un altro partecipante distingue il discorso sul prodotto in generale dal discorso sulla marca, specificando che l'oggetto di cui è questione in quel momento non è "gillette" ma è di un'altra marca. Per tutta la durata dell'interazione, l'oggetto di discorso costituito con l'uso del marchionimo "non viene mai esplicitato attraverso un nome comune (come rasoi, lamette ecc.), bensì, a livello verbale, tramite elementi deittici" (De Stefani 2008: 720).

È interessante che alcune tra le poche osservazioni pertinenti a proposito dell'integrazione di marchionimi nel discorso provengano da un volume sulla traduzione, che studia questioni correlate alla traduzione di (costrutti contenenti) nomi propri tra tedesco e francese. A conoscenza di chi scrive, Grass (2002) è l'unico a osservare il particolare comportamento sintattico dei nomi d'impresa, che entrano in nessi a valore locativo e, in altri casi, si combinano a un predicato che legittima un soggetto [+ umano].

\section{Conclusioni}

Una considerazione conclusiva a proposito dello stato della ricerca sui nomi di marca. L'ampia bibliografia di taglio linguistico sui marchionimi, elaborata soprattutto a partire dagli anni Settanta del Novecento, presenta essenzialmente tre limiti. In primo luogo, la maggior parte dei saggi studia i nomi di marca da un punto di vista squisitamente lessicale e/o diacronico, considerandoli indipendentemente dal contesto sintattico e concentrandosi perlopiù su aspetti formali, quindi in quella prospettiva filologica ed etimologica che è tipica degli studi d'impianto onomastico (si vedano per es. Koss 1996a; Ronneberger-Sibold 2004). L'attenzione si concentra sull'origine dei nomi, sulla tipologia grammaticale degli elementi che li costituiscono e sul loro statuto fonetico e morfologico, con l'obiettivo di classificarli secondo criteri formali o semantici. In secondo luogo, larga parte degli studi linguistici è dedicata a questioni puntuali; sono rare le opere che offrano visioni d'insieme. 
A questo proposito si possono ricordare i fondamentali Gabriel (2003) e Platen (1997), che però considerano unicamente i nomi di prodotto e le cui indagini sono, ancora una volta, di impostazione prevalentemente onomastica. In terzo luogo, non sono molti gli studi prettamente linguistici, situandosi la maggior parte dei lavori all'incrocio con altre discipline. In considerazione di questi presupposti bibliografici in parte lacunosi si elaboreranno, nella Parte II, le basi teoriche e metodologiche dell'analisi. 
Parte II

Fondamenti teorici e metodologici 



\section{Premesse teorico-metodologiche}

\subsection{Valore}

La marca, oggetto di questa ricerca, è un fenomeno moderno ma non del tutto nuovo. Nuovo è lo sguardo che la considera: ${ }^{1}$ uno sguardo marziano, di chi non ha a disposizione categorie ontologiche atte a classificare ma soltanto dati linguistici - enunciati, esempi testuali - che sono manifestazioni di rapporti sintagmatici e paradigmatici. Lo si è visto nei capitoli che precedono: con i marchionimi non è possibile partire da definizioni univoche e categorizzazioni plausibili. Lo stesso, del resto, vale pure per il nome proprio, di cui i nomi commerciali sembrano una declinazione. Conviene dunque prendere le mosse dalla già ricordata intuizione di Jakobson (cf. Parte I, §3.2) secondo la quale il "significato generale" di un nome proprio non può essere definito senza un riferimento al codice; nel codice dell'inglese, Jerry significa una persona chiamata Jerry (Jakobson 1957: 131). Di qui scaturisce l'osservazione di La Fauci (2010: 202) che guida questa analisi sperimentale: "Nome proprio non è una categoria lessicale ontologicamente definita, è un valore processualmente determinato che si realizza, secondo i diversi contesti, in una certa varietà di forme".

Innanzitutto va chiarito che cosa si intende qui per "valore" e quale procedura permette di stabilirlo. Il riferimento è a Ferdinand de Saussure (1916, in particolare i capp. "Identità, realtà, valori" e "Il valore linguistico"), più correttamente ai manoscritti rimasti inediti fino a tempi recenti (Saussure 2002). Il valore linguistico, fulcro della teoria saussuriana, vi appare strettamente legato all'altro fondamentale concetto di sistema: la lingua, per il linguista ginevrino, è un sistema

1 Si dà seguito all'invito di Saussure a sostituire, in linguistica, "la discussion des points de vue à celle des «faits», puisqu'il n'y a pas la moindre trace de fait linguistique, pas la moindre possibilité d'apercevoir ou de déterminer un fait linguistique hors de l'adoption préalable d'un point de vue" (Saussure 2002: 24-25). 
di valori relazionali; il valore non è nient'altro che un rapporto, una differenza, e si basa su un'opposizione (Saussure 2002: 70). Una forma, scrive Saussure, "non significa ma vale", implicando di conseguenza l'esistenza di altri valori (Saussure 2002: 28). Ogni segno esistente nella lingua ha valore essenzialmente negativo:

Considérée à n'importe quel point de vue, la langue ne consiste pas en un ensemble de valeurs positives et absolues mais dans un ensemble de valeurs négatives ou de valeurs relatives n'ayant d'existence que par le fait de leur opposition. (Saussure 2002: 77)

Studiare un'entità della lingua, sia essa una proposizione o un sintagma o un nome di marca, significa dunque studiare qualcosa che è parte di un sistema, che è inserito in un insieme di relazioni e possiede un valore perfettamente relativo. Il valore di una parola o di un'espressione non è solo la proprietà che essa ha di rappresentare un'idea (questa sarebbe la significazione), dal momento che nel sistema della lingua tutti gli elementi sono solidali e il valore dell'uno non risulta che dalla presenza simultanea degli altri.

Il Cours de linguistique générale espone un paragone con il sistema monetario:

per determinare che cosa vale un pezzo da cinque franchi, bisogna sapere: 1 . che lo si può scambiare con una determinata quantità di una cosa diversa, per esempio con del pane; 2 . che lo si può confrontare con un valore similare del medesimo sistema, per esempio un pezzo da un franco, o con una moneta di un altro sistema (un dollaro ecc.). (Saussure 1916: 140)

Nei vari casi si tratta di stabilire relazioni oppositive. È tipico del valore, osserva Saussure (2002: 335), mettere in relazione ciò che può essere scambiato con ciò che lo può sostituire.

Questo è il fondamento metodologico dell' analisi: studiare i nomi commerciali come espressioni linguistiche, che in quanto tali sono qualificate dalle relazioni combinatorie e commutative che le definiscono nei testi in cui ricorrono; ricercare il valore dei nomi commerciali, ossia il rapporto che li individua nella prospettiva duplice e interrelata delle relazioni in praesentia e in absentia, approfondendo le proprietà combinatorie che questi nomi manifestano di volta in volta nella proposizione 
(livello morfosintattico). Il risultato non sarà una tassonomia rigida $\mathrm{e}$ incrollabile, una categorizzazione a base ontologica, bensì una rassegna di alcuni dei valori dei marchionimi che si manifestano nei testi e dei modi con cui lo fanno.

\subsection{Funzione}

Secondo l'accostamento teorico delineato, lo studio linguistico deve mirare a determinare i "valori funzionali (cioè correlativi)" (La Fauci 2011: 277) di espressioni contenenti nomi commerciali. Si tratta pertanto di una prospettiva funzionale, così come indicato da La Fauci (2009: 35):

La qualificazione ["funzionale"] è da intendere nel senso che si attribuisce al concetto di funzione quando si dice, per es., che lo stato (solido, liquido, gassoso) sotto cui si presenta l'acqua varia in funzione della sua temperatura, cioè in relazione con essa, in dipendenza di essa. [...] Adottare un punto di vista funzionale significa vedere ogni elemento che ricorre in un nesso come manifestazione di una funzione sintattica. Con funzione sintattica, o relazione grammaticale di un elemento s'intende l'insieme dei rapporti da esso manifestati, rapporti con altri elementi e con l'insieme del nesso.

Nozioni fondamentali sono, in questo senso, predicato e argomento (cf. La Fauci 2009: 36), intesi proprio come concetti funzionali. A livello di nesso nominale (La Fauci 2009: 139-141) un nome - per riprendere la tradizionale suddivisione categoriale - costituisce il nucleo del nesso e vi svolge funzione argomentale, mentre si riconosce funzione predicativa ai modificatori di tale nome, per es. agli aggettivi. Il rapporto tra i due elementi che compongono il nesso scarpe Adidas sarà dunque un rapporto tra argomento (scarpe) e predicato (Adidas), come sarebbe nel caso di scarpe nuove. Allo stesso marchionimo, peraltro, si riconoscerà funzione argomentale quando ricorra come nucleo del nesso, con la possibilità di essere preceduto da articolo: Adidas (produce...), la Adidas, le Adidas (nuove). Per stabilire se un marchionimo manifesta 
un predicato o un argomento è insomma necessario valutare l'insieme di rapporti che costituiscono un nesso (La Fauci 2009: 37).

\title{
1.3 Studiare relazioni e ricercare opposizioni
}

La lingua "non ha un lessico prima di avere una sintassi" (La Fauci 2011: 33). Seguendo questa prospettiva si propone di approfondire il funzionamento testuale dei nomi commerciali abbandonando le sicurezze della lista - terreno di ricerca tradizionale degli studi onomastici per avventurarsi nei meandri del testo, che si costituisce come rete di relazioni, non come successione di entità ontologicamente e intrinsecamente determinabili. Il tentativo è riconoscere, nella sedimentazione restituita dalla lingua scritta, momenti di un processo del quale le parole (quindi anche i marchionimi) e i loro valori specifici sono non un presupposto ma tutt'al più un risultato:

\begin{abstract}
come i suoni in quanto elementi della lingua, così le parole non stanno al principio del processo linguistico. Ammesso che esistano, stanno eventualmente solo alla sua momentanea (e, a dire il vero, solo apparente) conclusione. Sono il prodotto precario dell'intersecarsi sistematico di relazioni. Lì, quanto alle parole, le trovano i lessicografi, nel loro benemerito e certosino lavoro di raccolta. Lì le vedono i parlanti, dimentichi (e come potrebbero non esserlo, nel flusso dei loro discorsi?) del silenzioso e inconsapevole lavoro di correlazione con cui essi costantemente le creano, anche quando pare loro, ingenuamente, solo di riutilizzarle. (La Fauci 2011: 33-34)
\end{abstract}

In questa prospettiva, non ai marchionimi si riconosceranno delle proprietà (sintattiche o formali), bensì ai costrutti in cui essi ricorrono e nei quali tali proprietà si manifestano, diventando correlativamente sperimentabili.

L'accostamento teorico, improntato a Ferdinand de Saussure, è mutuato da La Fauci $(2009 ; 2011)$, il quale concepisce la lingua come "processo $[\ldots]$ che opera sistematicamente per relazioni e differenze" (La Fauci 2011: 15). Si tratta di determinare, saussurianamente, i rapporti sintagmatici e paradigmatici, che permettono di "cogliere con 
precisione la natura sistematica della lingua, che (non lo si dimentichi) non è un oggetto ma una relazione" (La Fauci 2011: 276). Per l'indagine che si svolge in questo lavoro ciò significa analizzare, da un punto di vista morfosintattico, i contesti di ricorrenza dei nomi commerciali: con quali elementi si combinano (in praesentia)? Tali elementi - per es. determinatori, aggettivi, nomi comuni - sono passibili di essere sostituiti da altri o da Ø? Questo permette, dati due nessi formalmente simili o due nomi che ricorrono identici in contesti diversi, di stabilire se i valori (cf. Saussure 1916: 140; §1.1) manifestati da tali espressioni sono i medesimi. La lingua, infatti, è "processo incessante di creazione di valori”"(La Fauci 2011: 279):

Nella lingua non c'è nulla che abbia la propria ragione in se stesso: un elemento linguistico non è positivamente determinato nel suo valore da nessun suo carattere. Il suo valore si istituisce e si coglie solo in forma correlativa, per differenza. (La Fauci 2011: 275)

Questo accostamento è particolarmente fecondo nel caso dei marchionimi, con i quali le categorizzazioni di tipo semantico si rivelano spesso insoddisfacenti e imprecise. Lo si illustra attraverso alcuni esempi.

(1) Se penso che la Fiat ha comprato l'Alfa nel 1987 per soli 1.050 miliardi a tasso zero pagati in cinque anni a partire dal $1992 \ldots$ è stato un regalo: ci pensa? (LaR online $^{2}$ )

(2) Quello con l'ape sicuramente è un povero contadino che va a lavorare la terra, tu invece sei solo un povero vagabbondazzo a cui il paparino ha comprato l'alfa in modo che tutti i tuoi amici ti possano invidiare quando vai a farti l'apericena... $\left(<\right.$ www.lecceprima.it $\left.>^{3}\right)$

Di fronte a questa coppia di costrutti in cui ricorre, identico, il nesso "(X) ha comprato l'Alfa" (fatta la tara dell'aspetto puramente grafico della maiuscola o minuscola iniziale), un accostamento tradizionale, referenziale, si limiterebbe ad affermare che il marchionimo Alfa è riferito all'impresa in (1), a un'automobile in (2). Da una prospettiva sintattica

$2<$ ricerca.repubblica.it/repubblica/archivio/repubblica/2002/10/12/cosi-hannodistrutto-un-mito.html> (3.3.2015). Per le abbreviazioni delle fonti cf. infra, §3.

$3<$ www.lecceprima.it/cronaca/incidente-stradale/incidente-corigliano-d-otran to-16-ottobre-2014.html> (3.3.2015). 
attenta alle differenze, invece, si può osservare che il nesso contenuto in (1), e solo quello, trova un equivalente nel costrutto seguente:

\section{(3) Fiat ha comprato Alfa nel 1986. (online ${ }^{4}$ )}

In altre parole, vi sono contesti in cui il marchionimo Alfa può ricorrere sia preceduto, sia non preceduto da articolo, senza che ciò comporti una variazione del valore globale del costrutto (cf. La Fauci 2011: 275). In questo caso, la differenza tra Alfa e l'Alfa si rivela non-pertinente.

Pertinente è, invece, la differenza tra la Superga e le Superga nei brani che seguono:

(4) La Superga vende scarpe per lo sport e per il tempo libero che ormai sono diventate un simbolo per migliaia di adolescenti. ( $\mathrm{LaR})$

(5) Per chi ha la fortuna di trovare il suo numero: le Superga, a esaurimento, costano 45 mila lire. (CORIS STAMPASupplementi)

A una minima variazione formale corrispondono due valori differenti. Per contro, valori correlativi diversi si possono manifestare anche quando lo stesso articolo determinativo precede il marchionimo:

(6) Fortunatamente per loro la Fiat era parcheggiata a meno di cento metri. Salirono in fretta e ripartirono a tutta velocità verso l'uscita del paese. (CORIS NARRATTrRomanzi)

(7) Prima della marcia dei quarantamila Cesare Romiti era, per gli operai, una sorta di sergente di ferro del padronato reazionario, oggi se la Fiat compera l'Alfa è lui ad apparire in televisione e a colloquiare con gli operai come un fratello maggiore, di cui ci si può fidare. (LaR)

In entrambi gli esempi ricorre la Fiat come soggetto di una proposizione. Nel brano (6), però, l'articolo determinativo può alternare con quello indeterminativo (una Fiat), mutamento che cambierebbe il significato del costrutto ma non il valore del marchionimo, mentre nel caso di (7) una sostituzione plausibile - lo si è visto in (3) - è con determinatore zero: "Fiat compera Alfa". Al contrario, nel primo caso la cancellazione del determinatore sarebbe fortemente marcata. 
Nei brani che seguono, la preposizione articolata davanti al nome commerciale costituisce quella che si può definire variante libera:

(8) L'amministratore delegato della Fiat, Cesare Romiti, intervenendo all'assemblea degli industriali di Modena, è tornato sul tema alla vigilia dell'incontro che il presidente incaricato, Giuliano Amato, avrà con CGIL, CISL, UIL per completare il giro di consultazioni, in vista della formazione del governo. (CoLFIS)

(9) Nel leggere la relazione scritta, il dottor Pieri ha aggiunto due aggettivi che non comparivano nel testo distribuito al pubblico: interferenza politico-sindacale. Un'aggiunta che non è passata inosservata al principale imputato di quel processo, l'amministratore delegato Fiat Cesare Romiti, seduto in seconda fila tra le autorità. $(\mathrm{LaR})$

I nessi L'amministratore delegato della Fiat (8) e l'amministratore delegato Fiat (9), pur differenti dal profilo morfosintattico, stanno in rapporto di equivalenza (uguale valore), senza implicare con ciò che siano del tutto identici sul piano stilistico o semantico (cf. Harris 1952: 20).

\subsection{Non basta la semantica?}

Si è appena affermato che esiste una differenza tra la Superga e le Superga, tra la Fiat dell'esempio (6) e la Fiat di (7), mentre nel caso di (8) e (9) non vi è differenza tra gli attributi della Fiat e Fiat. A questo punto può sorgere la domanda: non sarebbe sufficiente far ricorso all'interpretazione, in un'ottica semantica, per rendere conto di tale diversità, parlando di azienda, scarpe, auto?

Innanzitutto non è possibile individuare referenti specifici per ogni caso, in particolare con i nomi di marca, che - lo si è notato già in questi primi esempi citati - si correlano a significati diversi a seconda dei costrutti in cui ricorrono. Ancor più importante, però, è il criterio analitico alla base di queste osservazioni: l'applicazione di un'etichetta specifica, determinata in maniera assoluta ma con un margine di soggettività (Superga $=$ 'scarpe' oppure 'azienda', Fiat = 'auto' oppure 'azienda'), si contrappone alla valutazione di differenze e somiglianze relative. Nei brani riportati le Superga non è la Superga, mentre l'Alfa 
equivale ad Alfa. Attraverso tale procedura correlativa non si affermano qualità assolute dei singoli nomi, bensì si portano alla luce, contrastivamente, proprietà - non positive ma oppositive - dei costrutti in cui ricorrono nomi di marca, prescindendo da partiti presi categoriali o referenziali.

Opposizioni e differenze su base correlativa hanno un notevole vantaggio metodologico rispetto a caratterizzazioni assolute: sono discrete (è innegabile che il Mercedes e la Mercedes siano effettivamente diversi) e sono meno interpretative (stabilire un referente extralinguistico preciso non è dirimente per l'analisi). Questo diventa cruciale nel momento in cui entrano in gioco conoscenze enciclopediche: un fenomeno normale con i nomi propri e paradigmatico con i marchionimi. Quando chi scrive usa la Fiat come nell'es. (1), alludendo a una ditta che produce automobili, oppure usa $i$ Levi's in riferimento a un paio di jeans, fa una presupposizione che ipotizza sia condivisa dal suo pubblico; enciclopedica perché appresa attraverso l'uso, la cui definizione non è reperibile nei dizionari (a volte neanche nelle enciclopedie) e che è legata a una cultura specifica, anche se certi stilemi superano le frontiere linguistiche e nazionali. Se si volesse stabilire in maniera precisa ognuno di questi referenti costituiti attraverso il discorso, inevitabilmente l'analisi perderebbe in coerenza.

L'aspetto semantico è chiamato in causa come strumento euristico per verificare la natura dei rapporti tra due o più costrutti. Alla stregua di Gross (1975: 31-32) il significato non è dunque inteso in maniera assoluta ma (cor)relativa: differenze o somiglianze di significato correlate a differenze o somiglianze combinatorie. Nell'esempio addotto da Gross, invece di affermare che Paul se renseigne auprès de Pierre contiene una componente "attiva" in relazione a Paul si osserverà che, rispetto a questo costrutto, la proposizione Pierre renseigne Paul sur cette question non presenta univocamente tale aspetto, dal momento che Pierre potrebbe aver dato delle informazioni a Paul senza esserne richiesto.

L'operazione che consiste nel paragonare dei costrutti, procedendo a una "valutazione differenziale di significato" (Gross 1975: 32 ), è per Gross più fruttuosa che non l'attribuzione di un significato magari con la sua etichetta descrittiva - a un solo costrutto o elemento 
linguistico. Tale operazione consente di mettere in evidenza differenze semantiche anche sottili e di paragonarle tra loro, ottenendo risultati discreti senza fare affermazioni assolute, come avviene quando si dà un nome a tali differenze.

\subsection{Notazioni}

L'impianto formale adottato nell'analisi è mantenuto volutamente leggero. Laddove necessario a fini di praticità, si utilizzano abbreviazioni per nozioni tradizionali della riflessione grammaticale: $N$ 'nome' o 'nesso nominale', Agg 'aggettivo', $V$ 'verbo'; inoltre Det 'determinatore' (cf. Gross 1977), Npr 'nome proprio', Nc 'nome comune'; infine Nmc per l'oggetto al centro della ricerca, il nome commerciale o nome di marca.

Si fa uso, con Gross (1975: 33), dei tratti "umano" e "nonumano", indicati con la notazione $[ \pm$ hum], da stabilirsi in relazione con un predicato che può selezionare argomenti specifici.

Giudizi di dubbia grammaticalità (il punto interrogativo in apice che precede le forme) segnalano che il costrutto, in particolar modo se confrontato con altri, ricorre con basse frequenze ed è, in ogni caso, fortemente marcato.

\section{Studi sintattici sui nomi propri (di marca)}

Gli studi dedicati alla "grammatica dei nomi propri"(Kolde 1995) e, più specificamente, alle ricorrenze di nomi commerciali in contesti proposizionali offrono alcuni spunti per l'analisi sintattica dei nomi di marca, che saranno esposti in questo capitolo. Al contempo si osservano però i limiti di tali ricerche, nelle quali, secondo un procedimento tipico della filosofia del linguaggio, lo studioso cerca di risalire al referente 
in base all'introspezione. I risultati che si ottengono sono inevitabilmente parziali, soprattutto in trattazioni brevi, come i capitoli che Van Langendonck (2007a) e Vaxelaire (2005) dedicano ai marchionimi: gli autori, infatti, finiscono per considerare soltanto le strutture più diffuse, che orientano di conseguenza l'analisi e la ricerca o creazione di esempi. Oltre a ignorare certe tipologie di costrutti si rischia di tacciare di impossibilità costrutti in realtà ben presenti nella lingua: Petit (2006: 692) mette l'asterisco a "Ma voiture Twingo est en panne" ma - perlomeno per l'italiano - non mancano ricorrenze di auto + marchionimo (cf. Parte III, §2.2.1).

Nell'ambito della sua tipologizzazione dei nomi propri, Van Langendonck (2007a: 236; v. inoltre 97) considera anche i nomi commerciali. Inserendo il nome Ford in una serie di proposizioni, egli identifica cinque categorie di referenti del nome commerciale:

1) fondatori di imprese: "Ford founded a car industry";

2) imprese: "Ford is a car company";

3) marche: "Ford is a familiar brand";

4) prodotti: "Ford is a good product";

5) esemplari di prodotti: "Jane bought a Ford yesterday".

La menzione della prima categoria, "fondatori di imprese", nell'ambito di una trattazione sui marchionimi è discutibile. Come prima cosa, non tutti i marchionimi derivano da patronimici (cf. Janner 2012a: 161); inoltre, se Ford vale come "fondatore di un'azienda", si tratta appunto di un patronimico, omonimo (per ragioni storiche) del nome commerciale.

Per lo studioso belga i diversi usi dello stesso nome sono collegati da processi metonimici (Van Langendonck 2007a: 236). Per descrivere il passaggio da patronimico a nome d'azienda e da nome d'azienda a nome di marca sarebbe peraltro forse più consono il concetto di omonimia, trattandosi di atti giuridici indipendenti. Del resto, quando si dà al proprio figlio il nome del proprio padre, tra i due nomi non si instaura un rapporto metonimico. Se dunque l'accostamento di Van Langendonck (2007a) parte da premesse condivisibili - un nome commerciale può manifestare significati differenti a seconda dei contesti di ricorrenza -, gli sviluppi del suo ragionamento lasciano aperti alcuni dubbi. 
Gli studi che rientrano sotto la dicitura di "grammatica del nome proprio" forniscono alcuni spunti per l'analisi combinatoria dei nomi commerciali e permettono, al contempo, di illustrarne caratteri specifici.

Come per i nomi propri prototipici, l'attenzione verte in primo luogo sul nesso nominale (Parte III, capp. 1 e 2): dal momento che la morfologia flessiva dei marchionimi è ridotta o nulla, ${ }^{5}$ numero e genere si manifestano principalmente negli articoli che precedono il nome. ${ }^{6}$

Una differenza importante tra marchionimi e nomi propri prototipici, in particolare antroponimi, riguarda i determinatori (v. Parte III, $\S 1.1$ ). Come osserva Petit (2006: 691-692), è largamente diffuso l'uso di nomi di marca preceduti da determinatore; con alcuni marchionimi, anzi, per es. BigMac o $F N A C$, l'uso senza determinatore è altamente improbabile. Grass (2002: 229) nota che determinatori ricorrono di rado con i nomi d'impresa (per l'italiano la situazione, come si vedrà, è differente), mentre ve ne sono quasi sempre, e di vario tipo, con i nomi di prodotto. Vanno distinti dagli altri i casi in cui l'articolo è "sintatticamente motivato" (Kolde 1995: 404; v. Parte III, §1.1.1), ossia ricorre in combinazione con un modificatore, per es. negli usi cosiddetti frazionanti del nome proprio: "Der junge Goethe war noch nicht so berühmt, wie es später der alte sein würde" (Kolde 1995: 404; con i nomi di marca si ha per es. "la Nike italiana"). Nei restanti casi bisogna interrogarsi sul valore specifico dell'articolo: si tratta di una variante libera (Parte III, $\S 1.1 .2)$ ? In quali contesti? Nei casi in cui manifesta un'opposizione, per cui solo in contesti specifici l'articolo può essere tralasciato (Parte III, $\S 1.1 .3$ ), va approfondito di che tipo di opposizione si tratti (cf. Kolde 1995: 404 sulla differenza tra Fulda = 'città' e die Fulda = 'fiume/nave').

Kolde (1995: 403-404) afferma di non occuparsi degli usi metonimici del nome proprio (preceduto da articolo), utilizzato per es. per

$5 \quad$ Non mancano casi di derivazione, spesso di carattere estemporaneo, che di frequente manifestano le stesse regolarità riscontrate con gli antroponimi (suffissi -iano/-ista). A quest'aspetto è dedicato il cap. 5 dell'analisi.

$6 \quad$ Kolde (1995: 402-403) studia genere e numero dei nomi propri nell'ambito della flessione. Qui se ne tratterà invece in relazione al nesso nominale e alla determinazione (Parte III, §1.2): un nome come Fiat, di per sé, non manifesta alcun genere; sarà manifestamente femminile se combinato a un determinatore di genere femminile ("la Fiat di Romiti") o quando siano presenti fenomeni di accordo al femminile ("Fiat è riuscita a..."). 
riferirsi a oggetti prodotti da chi ne porta il nome ("comprare un Picasso"): per lui si tratta infatti di nomi comuni. In questa sede, peraltro, ci si occuperà anche di tali strutture, se si vogliono definire usi metonimici quelli del nome d'azienda per il suo prodotto (le Adidas, la Peugeot): non interessa, infatti, lo studio dei marchionimi in quanto nomi propri "veri", utilizzati referenzialmente, bensì la descrizione delle differenti ricorrenze dei nomi commerciali.

In combinazione con i nomi propri, gli articoli indeterminativi mettono in campo un ventaglio di strutture, variamente definite dagli studiosi come "esemplari", "denominative", "metaforiche", "modalizzanti" (cf. Kolde 1995: 405, sulla scorta di Gary-Prieur 1991: 47-48). Bisognerà considerare che cosa comporti il ricorrere di articoli indeterminativi con i marchionimi.

Vi è poi la questione del genere (cf. Parte III, §1.2.1). Grass (2002: 230) correla il genere dei nomi commerciali a quello del nome generico relativo, chiamando in causa il fenomeno dell'ellissi: una Heineken sarebbe femminile perché implica un riferimento a birra, nome "cancellato" o "sottinteso". Si registrano peraltro diverse eccezioni, oltreché alcuni casi per i quali la motivazione del genere non è chiara. Nel corso dell'analisi (cf. Parte III, §1.1.3) si vedrà che il riferimento all'ellissi è problematico e non pare la soluzione migliore.

Un altro costrutto ricorrente vede la combinazione di nome comune e nome proprio (di marca), che svolge funzione attributiva o modificatrice rispetto al primo: "l'azienda Adidas", "la birra Peroni" (v. Parte III, cap. 2). Con i nomi propri prototipici, una struttura simile si incontra nei costrutti con designazioni di professioni o titoli (Herr Miller, Bäcker Otto Miller), che Kolde (1995: 406) definisce "Deskriptoren", interrogandosi al proposito sulla relazione sintattica tra i due nomi e confrontandoli con i "nomi propri complessi" del tipo "der Eiffelturm", "die Goethestrasse". In italiano, generalmente, tali nomi composti sono resi con strutture corrispondenti (cf. Grass 2002: 234, che confronta francese e tedesco): "der Fiat-Chef" (N1N2) equivale per es. a "il boss della Fiat" (N2 di art. N1), "il boss Fiat" (N2 N1) o, più raramente, "il boss di Fiat" (N2 di N1). Si pone quindi la questione di un'eventuale relazione parafrastica tra le diverse strutture. ${ }^{7}$

7 Qui si riconosce un'altra differenza tra nomi propri prototipici e marchionimi. Se vi è una somiglianza tra i costrutti metonimici Ugo veste Prada e Ugo ascolta 
In linguistica, in particolare di area francofona, le ricorrenze di nomi propri "non-nudi", ossia accompagnati da determinatori e/o modificatori (cf. La Fauci 2009: 143), sono studiate in merito alla questione se si tratti di usi "modificati" o no (sul concetto di "nome proprio modificato", rilanciato da Kleiber 1981, cf. le riflessioni critiche di Gary-Prieur 2005). La nozione è peraltro ambigua: vi è chi intende con essa ogni tipo di modificazioni sintattiche, ossia tutti i casi in cui il nome proprio ricorre accompagnato da determinatori e modificatori, e chi invece definisce "modificati" solo i casi in cui la modificazione sintattica determina pure una modificazione semantica, cambiando in certo modo la natura del nome proprio (cf. Gary-Prieur 2005: 53; Jonasson 2005: 67). Per i costrutti con marchionimi ci si occuperà delle variazioni morfosintattiche e solo secondariamente, in maniera correlata, di eventuali variazioni semantiche.

Kolde (1995: 407) sostiene che i caratteri specifici dei nomi propri si fanno più rari man mano che si sale nella gerarchia della grammatica. Si ritiene, però, che anche il livello proposizionale meriti uno studio, a cominciare dalle funzioni grammaticali di volta in volta ricoperte dai nomi di marca a livello di proposizione (cf. Parte III, cap. 4). Con i marchionimi in funzione di soggetto (Parte III, §4.1), per es., si pone la questione di un eventuale tratto [ + umano] del nome commerciale, legittimato dal predicato (Grass 2002: 231).

In relazione alla combinazione di marchionimo e predicato verbale, Grass (2002: 226-227) approfondisce quali "verbi supporto" selezionano i marchionimi, notando che, quando un nome di prodotto ricopre la funzione di oggetto diretto, il predicato dipende dalla classe di oggetti cui esso appartiene ("ich fliege Lufthansa", "ich drinke Warsteiner"). Per questa struttura, che in questo lavoro si definisce avverbiale (cf. Parte III, §4.4), sono utili gli spunti contenuti in Migliorini (1956), Folena (1964), De Mauro (1967).

Bach (lo stilista per l'abito, il compositore per l'opera), non è così, però, per i nessi nominali l'abito Prada e 'il concerto Bach, che necessita dell'inserimento di una preposizione per essere ben formato, mentre nel primo caso se ne può fare a meno. 


\section{I dati: criteri di raccolta e problemi}

Per la prima volta nell'ambito degli studi linguistici sui marchionimi, si propone in queste pagine una ricerca sperimentale ad ampio raggio, basata su osservazioni empiriche e di natura essenzialmente descrittiva. In questa prospettiva i dati linguistici non svolgono il ruolo di semplici pezze d'appoggio, a illustrazione di una teoria elaborata a priori; sono invece parte integrante della ricerca, che si delinea pertanto come corpus-driven, non corpus-based (cf. Tognini-Bonelli 2001). Ne emergono strutture che, pur comunissime per gli utenti della lingua, raramente sono prese in considerazione dai linguisti.

Nel suo nucleo portante, l'insieme di dati è mantenuto volutamente omogeneo, allo scopo di ottenere risultati coerenti. Questo determina la preferenza data a testi con scopi comunicativi informativi e non promozionali. I testi pubblicitari, principale campo d'indagine ai primordi degli studi linguistici sui marchionimi (cf. i saggi raccolti in Baldini 1987 e Chiantera 1989), sono qui considerati solo sporadicamente e in maniera contrastiva, in quanto caratterizzati da intenti apertamente perlocutivi e da sperimentalismo linguistico (cf. Zardo 1997: 36). Questa ricerca si fonda soprattutto sulla tipologia testuale giornalistica (quotidiani e riviste), che consente di indagare strutture ricorrenti nella lingua comune e presenta il vantaggio di un'ampia reperibilità di corpora in rete. Agli scritti giornalistici si aggiungono esempi di altre testualità, soprattutto letteraria (v. Parte III, cap. 7).

I dati analizzati sono stati ricavati dalle seguenti fonti:

1) rivista per consumatori Spendere Meglio $^{8}$ (= SM; si riportano fascicolo e annata): spoglio manuale completo di alcune annate (2003-2010); 
2) corpora La Repubblica (= LaR), Corpus e Lessico di Frequenza dell'Italiano Scritto ${ }^{10}$ (= CoLFIS), CORpus di Italiano Scritto ${ }^{11}$ (= CORIS): ricerche mirate;

3) Lessico di frequenza dell'italiano parlato $^{12}$ (= LIP; si indicano città, tipologia e numero del testo): spoglio elettronico. ${ }^{13}$

Consapevoli delle necessarie limitazioni insite nei criteri di spoglio dei corpora e della discutibilità di alcune scelte, si provvede qui a renderne conto.

Punto di partenza e, al contempo, obiettivo della descrizione, i nomi commerciali sono presentati non come una categoria definita ma come un'ipotesi di lavoro. È stato osservato (cf. Parte I, §3.1) che manca una definizione univoca di "marchionimo" in linguistica, cosicché non è possibile stabilire in maniera netta quali elementi comprenda tale classe. L'indeterminatezza della categoria, non risolvibile nell'immediato, comporta una serie di problemi nella ricerca di dati: come determinare, all'interno di vaste raccolte di testi, i costrutti rilevanti per l'analisi? Cosa inserire nell'interfaccia di ricerca, di quali nomi tenere conto nello spoglio manuale dei testi? ${ }^{14}$ Se non vi saranno dubbi a considerare Barilla e Adidas come marchionimi, andando verso i margini di un ideale schema di prototipicità si incontreranno nomi quali Einaudi, Raitre, P2 - marchionimi? Si tratta indubbiamente di brand, ma certo diversi dai primi citati. Ancora, come valutare i casi di Benetton o Foppapedretti in quanto nomi di squadre sportive? In questo caso

9 Raccoglie le annate 1985-2000 del quotidiano ed è costituito da circa 380 milioni di token. Cf. <sslmit.unibo.it/repubblica> (24.9.2014); accessibile previa registrazione.

10 <esploracolfis.sns.it/EsploraCoLFIS/> (24.9.2014). Un problema di questo corpus è che non tutti i testi sono disponibili integralmente.

11 <corpora.ficlit.unibo.it/TCORIS/> (24.9.2014). Si sono ritenuti in particolare gli esempi più recenti, quelli indicati con la sigla MON 'monitoraggio', seguita dagli anni in cui è stato effettuato l'aggiornamento dei dati.

$12<$ badip.uni-graz.at> (24.9.2014).

13 Un confronto sistematico delle strutture ricorrenti in fonti scritte e orali non rientra tra gli obiettivi del lavoro.

14 Com'è ovvio, anche sulla base dei problemi delineati nei capitoli che precedono, la situazione ideale - una taggatura uniforme dei marchionimi che permetta di ricercarli nelle banche dati - è destinata a restare utopica ancora a lungo. 
si è deciso di considerarli semplici omonimi dei nomi commerciali e di non trattarne nell'analisi, dato il loro funzionamento sintattico eterogeneo rispetto ai marchionimi. Similmente si è proceduto per nomi di testate giornalistiche, da Il Giornale a L'Espresso, che, pur essendo nomi propri e nomi di marca, costituiscono una classe a sé.

La consapevolezza della varietà di costrutti in cui ricorrono marchionimi si associa all'obiettivo di presentare un'analisi che coinvolga un grande numero di marche diverse. Se, come osserva Jerzy Pelc (cit. in Vaxelaire 2005: 15), non si può costruire una teoria dei nomi propri avendo come unici esempi John e Fido, parimenti non è pensabile uno studio dei marchionimi che si basi solo su Ford e Coca-Cola. Per questo la ricerca comprende non solo i marchionimi che hanno il maggior quoziente di brand awareness, ovvero i primi che vengono in mente, ma, per es., anche larga parte dei 500 brand selezionati dalla sezione italiana di Superbrands ${ }^{15}$ agenzia internazionale che identifica e premia le migliori marche presenti sul mercato (italiano). Altri nomi commerciali sono stati ricavati dalle liste di lemmi relative al corpus $L I P,{ }^{16}$ in cui sono contrassegnati i "nomi di ditta" ${ }^{17}$ In una fase ulteriore dell'analisi sono state eseguite ricerche avanzate nel corpus LaR facendo uso della variabile [pos="NPR"] ('parte del discorso = nome proprio').

Non si ignora il fatto che i dati provengono da corpora piuttosto datati (LaR comprende testi dal 1985 al 2000, CoLFIS e LIP risalgono all'inizio degli anni Novanta, mentre CORIS è stato messo in rete agli inizi del Duemila ed è aggiornato tramite un corpus di monitoraggio ${ }^{18}$ ). Più che influire - si ipotizza - sulle strutture linguistiche nelle quali ricorrono i nomi di marca, questo comporta delle conseguenze quanto alla frequenza con cui certi marchionimi ricorrono e ancor più non ricorrono, per es. quelli legati a Internet e alle tecnologie informatiche. Per

15 Cf. <www.superbrands.com/it/> (21.1.2015). La lista, del 2012, è reperibile sotto <www.yumpu.com/it/document/view/26903030/lista-dei-500-brands-selezionati-pdf-superbrandsit $>(15.12 .2014)$.

16 Cf. <badip.uni-graz.at/it/lista-dei-lemmi> (24.9.2014). Strumento utile ma spesso lacunoso.

17 Per le categorie distinte nell'annotazione del corpus cf. < badip.uni-graz.at/it/ classi-di-parola> (24.9.2014).

18 L'ultima volta nel 2011. Cf. <corpora.ficlit.unibo.it/coris_itaProgett.html> (3.11.2014). 
ovviare a questo problema, allo spoglio dei corpora sono state aggiunte ricerche puntuali nell'archivio digitale de La Repubblica. ${ }^{19}$

Nei dati sono stati corretti refusi evidenti e si sono segnalati in corsivo i nessi pertinenti all'analisi. Dove non indicato diversamente (con [titolo]), i brani provengono dal corpo dell'articolo.

L'analisi aspira a seguire un metodo nomotetico ma è idiosincratica: procede infatti tanto per campioni di marchionimi quanto per campioni di testi. Si precisa che quanto si afferma è valido solo per gli esempi analizzati, che non sono rappresentativi di tutta la lingua italiana; non è pertanto possibile generalizzare i risultati ottenuti.

19 <ricerca.repubblica.it> (24.9.2014). 

Parte III

Analisi morfosintattica 



\section{Introduzione}

Si riportano ora i risultati dell'analisi combinatoria dei dati. Questa sezione si struttura in quattro capitoli centrali, relativi a diversi livelli di analisi, più tre capitoli che completano il panorama accennando ad aspetti correlati.

I primi due capitoli sono incentrati sulle due funzioni (v. Parte II, §1.2) che manifestano i nomi commerciali entro il nesso nominale: argomentale (cap. 1) e predicativa (cap. 2). Le questioni principali relative agli usi argomentali - un Rolex d'oro, le Nike, l'Alfa Romeo di Pomigliano d'Arco - riguardano determinazione, genere e numero. Quanto agli usi predicativi, ossia giustapposizioni come la ditta Barilla, le sigarette Marlboro, il pilota Mercedes, che già Migliorini (1927:341) descrive come costrutti tipici dell'ambito commerciale, ci si domanda in quali casi il marchionimo rappresenti una modificazione attributiva di tipo aggettivale e in quali, invece, una modificazione di tipo denominativo, in cui il nome comune è in realtà il nucleo del nesso.

Il cap. 3 presenta i nessi in cui nomi commerciali sono preceduti da una preposizione.

Nel cap. 4 sono studiate le ricorrenze di nomi commerciali nella proposizione. L'analisi verte sulle funzioni grammaticali che ricoprono a livello proposizionale: soggetto, oggetto diretto e indiretto, nome del predicato; sugli usi cosiddetti avverbiali (Il diavolo veste Prada) e su altre tipologie di costrutti ricorrenti (per es. rialzo targato Fiat).

Nel cap. 5 sono considerate brevemente le modificazioni morfologiche dei marchionimi, non molto frequenti e spesso estemporanee.

Il cap. 6 verte su alcuni usi metaforici dei nomi commerciali; vi si studiano gli arricchimenti semantici di nomi quali Barbie o Baci Perugina nei diversi contesti sintattici di ricorrenza. 
Nel cap. 7 si analizzano le numerose citazioni marchionimiche di un romanzo italiano - Tre metri sopra il cielo di Federico Moccia - in prospettiva morfosintattica e sistemica.

Il Consuntivo dell'analisi riepiloga le varie strutture morfosintattiche, offrendo una riflessione sugli aspetti combinatorî e funzionali emersi nel corso dell'analisi. 


\title{
1. Nessi nominali: usi argomentali
}

\author{
Introduzione
}

(1) Luraghi, cosa ne pensa della proposta Ford? "La Ford è potente, ricca, ben radicata in Inghilterra e Germania. Quasi certamente saturerà le produzioni di Arese e di Pomigliano, ma addio Alfa Romeo. La Ford farà delle Ford, anche se userà il nome Alfa". (LaR)

La Ford, delle Ford: ecco un nome commerciale utilizzato, in due costrutti diversi, come nucleo di un nesso nominale. Sono usi che si definiscono argomentali (v. Parte II, §1.2), in opposizione agli usi predicativi che saranno analizzati nel cap. 2. In questo capitolo se ne studiano alcuni aspetti relativi a determinazione e modificazione: presenza/ assenza di determinatori e loro pertinenza $(\S 1)$; categorie di genere e numero, che si manifestano nei determinatori e/o attraverso fenomeni d'accordo (\$2); quanto alla modificazione, si analizza la relazione dei nomi commerciali con attributi e apposizioni (§3).

Tali questioni, seppur in misura ridotta, hanno ricevuto l'attenzione di linguisti che si sono occupati dei marchionimi nei contesti di ricorrenza (cf. per es. Van Langendonck 2007a: 236; Vaxelaire 2005: 345-351). Se ne rende conto in questa parte introduttiva.

Alcuni studiosi osservano che nel caso degli usi nominali - perlomeno in inglese e francese - la determinazione sembra manifestare una differenza referenziale, che si riflette anche nella categorizzazione (v. Parte I, §3.2): i nomi di marca e d'impresa, che sono simili ai nomi propri, verrebbero utilizzati senza articolo; esso sarebbe invece obbligatorio con i nomi di prodotto, che sono nomi comuni (Renault $=$ nome dell'impresa; une Renault = prodotto dell'impresa; cf. Vaxelaire 2005: 346; Jonasson 1994: 29). Si rivela così un luogo comune duro a morire negli studi onomastici, per il quale i nomi propri sarebbero intrinsecamente determinati. 
Esempi reali rivelano peraltro che la situazione è più complessa. In italiano non pare possibile tracciare un discrimine tra due usi differenti di Ford o Mercedes sulla base della presenza o assenza di articolo: entrambi gli esempi di Algeo che riporta Vaxelaire (2005: 346), "Onassis sold Ford and bought Volkswagen" (con nomi di marca) contrapposto a "Onassis sold the Ford and bought a Volkswagen" (con nomi di prodotto), possono presentare articoli nella traduzione italiana (cf. §1.1.2). ${ }^{1}$ Quanto ai nomi cosiddetti di prodotto, 1'“obbligatorietà" dell'articolo è in realtà mitigata non solo dall'uso linguistico dei produttori che, come nota lo stesso Vaxelaire (2005: 349-350), vogliono mantenere uno statuto propriale ai nomi dei loro prodotti (cf. §1.1.4), ma anche e soprattutto dal loro ricorrere in strutture sintattiche specifiche - in generale: usi con valore plurale (indeterminato) - nelle quali la presenza di determinatori è non certo esclusa ma perlomeno marcata (come si vedrà al §1.1.3). Ė quindi necessario analizzare i dati senza partire da schemi preconcetti, quale può essere la distinzione aprioristica tra nome di marca e nome di prodotto.

Mentre nella bibliografia le tipologie di determinatori combinati ai marchionimi sono raramente tematizzate (si riportano quasi solo esempi con articoli determinativi e indeterminativi, senza approfondirne differenze d'uso), la questione relativa a numero e genere dei nomi commerciali ( $\$ 1.2 .1$ e 1.2.2) è fatta oggetto di riflessione e collegata spesso all'aspetto referenziale. L'articolo è il principale luogo deputato alla manifestazione di genere e numero del marchionimo; ma a cosa sono correlati? Sono numero e genere di un nome comune "sottostante", cancellato in un processo d'ellissi (cf. Grass 2002; Gross 1977), per cui si dirà la Heineken perché si pensa (o si dice) la birra Heineken? Anche qui, in realtà, manifestazioni formali apparentemente simili - la Fiat, la Levi's, i Levi's - nascondono fenomeni combinatorî differenti, quindi diversi valori funzionali del nome commerciale.

Lo stesso vale per gli es. di Jonasson (1994: 29): "Renault vient d'acheter Citroën" vs. "Paul vient d'acheter une Citroën". Si sorvola qui sul fatto che una possibile traduzione italiana del primo esempio portato da Algeo, "Onassis sold Ford and bought Volkswagen", comporterebbe articoli determinativi, permettendo eventualmente di differenziare il significato sulla base del tipo di determinazione. 


\subsection{Determinazione}

Nei dati si osserva che i nomi commerciali ricorrono sia preceduti, sia non preceduti da un determinatore. ${ }^{2}$ Come si caratterizzano tali presenze e assenze in una prospettiva sistemica? Quali sono i valori e le funzioni della determinazione in combinazione con i nomi commerciali?

Per comprenderlo è utile studiare, in un'ottica saussuriana, i rapporti che i determinatori eventualmente presenti manifestano tanto sul piano sintagmatico, con altri elementi della proposizione, quanto su quello paradigmatico, con elementi passibili di sostituirli nello stesso contesto e con l'assenza di determinazione. ${ }^{3}$

\subsubsection{Determinatori sintatticamente motivati}

L'indagine prende avvio dai contesti in cui la determinazione è sintatticamente motivata (cf. Kolde 1995: 404), in particolare dalla concomitante presenza di una modificazione (cf. Vaxelaire 2005: 111). ${ }^{4}$

Se il nome commerciale è preceduto da un aggettivo possessivo, si osserva la presenza di un articolo correlato, perlopiù determinativo:

(1) Sabato notte, dopo quasi cinque ore di guida, Antonino Russo ha fermato il suo "Fiat 190" a Viverone, poco oltre il casello di Albiano, dopo aver percorso quasi metà della bretella Ivrea-Santhià. (CoLFIS, quotidiano)

2 Si tratta in prevalenza di articoli. Esempi con altri determinatori sono più rari: "Disponibili con motori da 1.300, 1.500 , e $1.600 \mathrm{cc}$ a benzina e da $1.900 \mathrm{cc}$ turbodiesel, queste Delta meritano un attento esame" (CoLFIS, periodico); "Che cosa è, dunque, questa nuova Fiat che ha risvegliato l'interesse degli Italiani per un prodotto nazionale?" (CoLFIS, periodico).

3 In questo paragrafo l'attenzione si concentra sui determinatori, con alcuni accenni ai tratti di genere e numero nella misura in cui sono funzionali all'argomentazione. Per una trattazione dettagliata di numero e genere si rimanda al §1.2.

4 Gli usi argomentali sono studiati primariamente in nessi nominali ma anche in nessi preposizionali (per questioni specifiche legate alla preposizione e ai costrutti relativi si veda il cap. 3 dell'analisi). 
(2) Mirzeta che ha problemi anche al braccino sinistro stringe tra le mani la sua Barbie, una Barbie anche lei scampata alla guerra con gli abiti sdruciti e i capelli stopposi. (LaR)

(3) "Quando mi sono accorto, all'inizio dell'ultima tappa, che mai sarei riuscito a raggiungere Auriol e la sua Lancia, ho deciso che avrei corso solo per i punti del secondo posto". (CoLFIS, quotidiano)

Negli esempi seguenti, invece, la presenza dell'articolo è correlata al ricorrere di un aggettivo qualificativo, preposto (4-6) o posposto (7-9) al nome commerciale: ${ }^{5}$

(4) La deliziosamente insopportabile Barbie dispone quest'anno di una shopping arcade, di una villa hollywoodiana e di una piscina tropicale. (LaR)

(5) Gli spolverini sono del tipo "crociera a Miami”, con accostamenti che qualcuno ha definito americani, e la donna diventa una docile Barbie, con il grande fiocco in vita, le piume nei capelli, i bermuda dai colori al neon [...]. (LaR) ${ }^{6}$

(6) Mega acquisizione a sorpresa nel mondo degli articoli sportivi: la tedesca Adidas rileverà la Salomon di Grenoble, famosa in tutto il mondo soprattutto per la sua attrezzatura sciistica. (CORIS STAMPAQuotidiani)

(7) L'indice Nikkei perde il $10 \%$, è affondato dal crollo dei mercati di sbocco del made in Japan, dal micidiale rialzo dello yen, dagli avvisi di tagli agli utili della Sony e altri colossi asiatici come la Samsung coreana. (CORIS MON2008_10)

(8) Togliete dalle mani dei vostri bambini le "Barbie" e le "Cindy" (la "Barbie" inglese) e tutti i giocattoli che rendono i bambini "prematuramente adulti", hanno tuonato le supreme autorità ecclesiastiche d'Inghilterra. (LaR)

(9) I best seller sono gli stessi, naturalmente: attorno a una Barbie sempre più rosa, ricca, capricciosa e accessoriata e a un "nucleo storico" fatto di Lego, Cicciobello e Playmobil ruota un universo di successi stagionali. (CoLFIS, quotidiano)

Oltre agli aggettivi, anche altri modificatori, funzionalmente equivalenti (cf. La Fauci 2009: 153), ingenerano il ricorrere di articoli e determinatori. Per es. nel costrutto (2), "una Barbie anche lei scampata alla guerra", con un attributo correlabile a una proposizione subordinata

$5 \quad$ Per la questione dell'ordine cf. $§ 1.3 .1$.

6 Sull'uso metaforico di Barbie v. infra, cap. 6. 
relativa (cf. La Fauci 2009: 154): "una Barbie che è anche lei scampata alla guerra". ${ }^{7}$ Così pure nell'esempio seguente:

(10) La Vespa della Piaggio, progettata nel 1945 da Corradino D'Ascanio, e la Lambretta disegnata nel 1947 da Cesare Pallavicino per l'Innocenti, non sono stati esclusivamente dei grandi successi commerciali: hanno costituito un simbolo estetico che andava al di là degli aspetti strettamente pratici del mezzo di trasporto. (CORIS STAMPASupplementi)

Nei brani che seguono la presenza dell'articolo è motivata da una relativa esplicita:

(11) "Small Soldiers" è anche un racconto di formazione. La sequenza più emblematica è quella in cui l'eroina adolescente distrugge, in evidente stato d'estasi, le barbie che Hazard e i suoi commandos hanno arruolato come ausiliarie, animandole e trasformandole in spietate guerriere. (LaR)

(12) Viene in mente il vecchio professor Valletta della Fiat. I politici, subito dopo la guerra, avevano escluso la possibilità di dare vita in Italia a una grande industria dell'automobile. Valletta, dopo averli ascoltati a Roma, se ne tornò a Torino e si mise a costruire la Fiat che oggi conosciamo. (CoLFIS, periodico)

Anche i complementi, in particolare di specificazione (cf. La Fauci 2009: 159-160), ricorrono in correlazione con un determinatore. Si vedano l'es. (10), La Vespa della Piaggio, e i seguenti:

(13) Da semplice sgabello per imprese decotte la Ribs si è trasformata in una finanziaria per il settore agro-industriale. Con l'incarico di finanziare gli imprenditori, compresi i grandi gruppi, come l'Eridania (Montedison), la Yomo (famiglia Vesely di Milano), la Parmalat di Calisto Tanzi, la Cirio di Sergio Cragnotti. (CORIS STAMPAQuotidiani)

(14) Tre delle berlinette italiane nei primi 5 posti, con Auriol davanti a Sainz, poi Kankkunen a precedere la Ford di Delecour e il giovane Bugalski in quinta posizione, seguito dalle Mitsubishi di Salonen e Nissan di Chatriot. (CoLFIS, quotidiano)

(15) Nel 1998 è stato annunciato un rinnovamento: la Barbie del nuovo millennio starà in piedi, il seno e fianchi saranno leggermente ridotti e la vita appena allargata, ma comunque sarà sempre ben diversa da Action Woman. (LaR)

7 Un es. con un altro determinatore: "[la] concorrente numero uno della Segafredo, ossia quella Lavazza che da anni ambienta in Paradiso gli spot sul caffe" (LaR). 
(16) Ad agosto 2004, Mercolli acquista un cellulare Ericsson T 230 alla Migros di Sant'Antonino. (SM 6/05)

(17) Il logo della Migros di Lucerna "Dalla regione. Per la regione" (Aus der Region. Für die Region) è presente su 389 prodotti. (SM 3/04)

In questi costrutti - con l'eccezione di La Vespa della Piaggio - il complemento di specificazione introduce una modificazione restrittiva, che delimita il riferimento del marchionimo in particolare in senso spaziale (le filiali di una catena: "alla Migros[, quella] di Sant'Antonino") o temporale ("la Barbie[, quella] del nuovo millennio").

I costrutti visti sinora contengono perlopiù articoli determinativi. La situazione è diversa quando ricorrono articoli indeterminativi:

(18) Insomma, Marchionne, che tornerà in Italia lunedì, considera incompatibile il nostro modello di relazioni industriali con una Fiat che ambisce a giocare sulla piattaforma globale. (CORIS MON2008_10)

(19) In siffatto clima prende corpo il dialogo con una Ford che ha tutto l'interesse a trovare un alleato per risolvere i suoi non pochi problemi in Europa. (LaR)

(20) Sempre in buona posizione Innocenti e Maserati, tornando alle altre marche estere si fa notare ancora una volta la Seat che ha già scavalcato il 2 per cento dell'intero mercato, mentre Ford e Opel appaiono stabili nelle loro posizioni, separate da una Citroen che si difende bene grazie al suo modello di punta, la $\mathrm{BX}$, oltre 300.000 unità prodotte in due anni, circa 30.000 delle quali vendute in Italia in 18 mesi. (LaR)

(21) La Guinness con la conquista della Distillers ha rafforzato enormemente la sua catena di distribuzione, si è impossessata di marche vincenti di pregiatissime bevande alcoliche e di proprietà immobiliari di immenso valore. Ma per Ernest Saunders, il guru della finanza al quale la famiglia irlandese che iniziò con la birra, aveva affidato la gestione dell'impero di una Guinness ormai estesa in tutti $i$ settori compreso quello delle banche, è in libertà provvisoria. (LaR)

(22) “[..] Gli investimenti che servono sono questi destinati alla formazione poiché nel tempo generano posti di lavoro". Una Fiat attenta alla formazione è una Fiat che si mette al passo e che vuole fare come hanno fatto altri. (CORIS STAMPAQuotidiani)

(23) Riesce ad immaginare un Sudafrica libero, con banche e società minerarie nazionalizzate, ma con una Ford tuttora in attività? "Perché no, le banche e 
le miniere sono nazionalizzate qui nello Zambia, eppure qui sono anche attive società estere". $(\mathrm{LaR})^{8}$

(24) Alcuni anni fa, racconta Ottone, Agnelli aveva già risposto al quesito, affermando che una Fiat non italiana non sarebbe concepibile. (CORIS STAMPA Quotidiani)

In questi esempi il nome commerciale si combina a un articolo indeterminativo ed è seguito da una subordinata relativa (18-20) o da un'espansione attributiva (21-24). Si ha qui a che fare con quelli che GaryPrieur (1991) definisce "usi modalizzanti" del nome proprio.

Non solo i determinatori che precedono il nome commerciale sono qui necessari, in quanto correlati al ricorrere di modificazioni a valore restrittivo (cf. La Fauci 2009: 154); il fatto che appaiano articoli indeterminativi è anch'esso legato al particolare tipo di struttura, è costitutivo delle costruzioni cosiddette modalizzanti al pari delle espansioni che seguono il nome proprio (cf. Gary-Prieur 1991: 47). Al proposito, in relazione ai nomi propri prototipici, vi è chi parla di "uso frazionante" del nome proprio (per es. Jonasson 2005: 69; Vaxelaire 2005: 117), mentre Gary-Prieur (1991: 49-50) vi riconosce "immagini" o modi diversi di guardare allo stesso referente; immagini che, peraltro, possono avere carattere ipotetico, come in (23-24): "una Fiat [che sia] non italiana".

Chiaramente non basta la presenza della struttura un(a) $N m c+$ modificatore perché si abbia a che fare con un uso modalizzante del nome commerciale. Tale interpretazione è legata, tra il resto, al fatto che l'articolo indeterminativo non è intercambiabile con quello determinativo. ${ }^{9}$ Si riconoscono costrutti $u n(a) ~ N m c+$ modificatore non modalizzanti, nei quali l'attributo che segue il nome ha funzione non-restrittiva (cf. La Fauci 2009: 154):

(25) Una Fiat vecchia maniera: solida, pratica, funzionale e anche bella. Una macchina che la rete del gruppo torinese non avrà problemi a vendere. (CORIS STAMPAQuotidiani)

8 L'esempio, si noti, contiene lo stesso tipo di costruzione analizzata, ma con un toponimo: "un Sudafrica libero".

9 In un certo senso, laddove si può riconoscere tale interpretazione essa vale come indice del valore propriale del nome che ricorre nella struttura. 
Una Fiat vecchia maniera, che apre l'articolo di giornale, si riferisce a un modello di automobile della casa Fiat - v. "Una macchina", nella frase successiva -, descritto peraltro già nel titolo: "Arriva l'auto universale Palio Weekend". Qual è la differenza con "Una Fiat attenta alla formazione" (22)? Appunto il carattere non restrittivo ma descrittivo del modificatore; si potrebbe dire: "questa Fiat è vecchia maniera", implicando che ne esistano di diverse. L'intero nesso "Una Fiat vecchia maniera" funge da predicazione (nominale) di un altro nome: "questa Fiat, la Palio Weekend, è vecchia maniera". Nell'es. (22), invece, l'attributo attenta alla formazione non modifica "una" Fiat tra le altre e in opposizione a esse.

L'alternanza tra i due tipi si incontra anche in questa coppia di esempi:

(26) Ieri mattina è stata presentata la nuova Fiat, la Tempra, al presidente Cossiga. (LaR)

(27) Allora non crede che la nuova Fiat di Romiti possa essere cambiata? "Quanto la Fiat sia diversa dal passato lo vedremo tra qualche mese. [...]”. (LaR)

In (26) la nuova Fiat è posto su un piano d'identità con l'inciso la Tempra che segue: "la Tempra", si potrebbe affermare, è "la nuova Fiat" di cui è questione. Non è così per la nuova Fiat di Romiti, perlomeno non in questa proposizione: 1'unica identità si può stabilire con "la Fiat" tout court (si veda la seconda parte del brano).

Pure nei seguenti casi il modificatore, preposto o posposto al marchionimo ${ }^{10} \mathrm{e}$ combinato a un articolo indeterminativo, non ingenera un'interpretazione modalizzante:

(28) Firmino salì in camera sua. Fece una doccia, si rase, indossò un paio di pantaloni di cotone e una Lacoste rossa che gli aveva regalato la sua fidanzata. (CORIS NARRATRomanzi)

(29) Entro pochi giorni, fra tutti coloro che avranno inviato i tagliandi, sarà sorteggiata una scattante Fiat Brava versione 1.4 super. (CORIS STAMPAQuotidiani)

10 Sul diverso ordinamento di nome e modificatore si rimanda ancora una volta al $\S 1.3 .1$. 
(30) Per lo sfizio di farsi vedere in giro a bordo di una fiammante Ferrari c'è gente disposta a pagare da 20 a 50 mila euro. E poco importa se la Rossa è taroccata come i Rolex venduti a Bangkok o le Lacoste che si trovano sulle bancarelle di Napoli. (CORIS MON2008_10)

In questi casi l'articolo indeterminativo sta in opposizione a quello determinativo. Anche qui l'alternanza con determinatore zero non si dà, sia per la struttura del nesso (presenza di modificatori), sia per il valore specifico del nome commerciale, che sarà approfondito nel §1.1.3.

\subsubsection{Presenza e assenza di determinatori come varianti liberamente alternanti}

I contesti in cui la determinazione costituisce variante libera, per prendere a prestito un concetto della fonologia, sono più numerosi di quelli in cui la sua presenza risponde a una contrainte di tipo sintattico. Dato che la determinazione come alternanza libera emerge con particolare intensità in relazione ai marchionimi (cf. Zardo 1997: 28-30), si potrebbe sostenere che tale fenomeno è caratteristico dei nomi commerciali e li distingue rispetto ad altri nomi, propri e comuni; si pensa in particolare ai nomi propri personali, con i quali l'alternanza tra presenza e assenza di determinatori è diatopicamente marcata.

Per studiare i casi in cui lo stesso nome commerciale ricorre sia preceduto, sia non preceduto da articolo (per es. Lavazza e la Lavazza), gli usi non modificati offrono le migliori condizioni di osservazione (cf. La Fauci 2009: 197).

(1) ROMA. Galbani chiude impianti, ma non licenzia. (CORIS STAMPAQuotidiani)

(2) È deciso: la Galbani chiude. Ma senza traumi per i lavoratori. (CORIS STAMPAQuotidiani)

In funzione di soggetto, legittimato dal predicato verbale chiudere, il marchionimo Galbani ricorre in (1-2) sia senza, sia con articolo. L'alternanza tra nome nudo e nome preceduto da articolo determinativo femminile singolare è frequente in tali contesti, come si osserva nelle coppie di esempi seguenti: 
(3) Eccovi una selezione di prodotti specifici per la bellezza del vostro seno. Biotherm propone Biofermeté seins fermes [...]. (CoLFIS, periodico)

(4) Per idratare a fondo la pelle del viso la Biotherm propone Actif Hydratant. (CoLFIS, periodico)

(5) Apple preferisce lasciare libertà di scelta ai clienti. Nelle confezioni dell'iPod ci sono le avvertenze sui rischi da volume alto, ma non si chiarisce quale sia la soglia. (CORIS MON2008_10)

(6) E la Apple si prepara a destabilizzare anche il cinema, dopo avere conquistato il campo della musica. (CORIS MON2005_07)

(7) Adidas non ha badato a spese per ringiovanire (e non si parli di lifting) il più grande [pugile] e inserirlo in uno spot. (CORIS MON2001_04)

(8) Continua il calvario di Jonah Lomu, l'asso degli All Blacks colpito da una rara malattia renale. [...] Unica buona notizia: l'Adidas gli ha rinnovato la sponsorizzazione. (CORIS MON2001_04)

(9) La campagna "Farmaci liberi" fa seguito a quelle per la vendita del latte in polvere per l'infanzia a prezzi europei e di prodotti senza glutine per celiaci con le quali Coop si è già attivata, dimostrando che è possibile ridurre i prezzi pur garantendo un'alta qualità dei prodotti. (CORIS MON2005_07)

(10) A differenza degli altri supermercati, la Coop presenta un livello di marketing che, accanto alla tradizionale pubblicità, vede la presenza dei soci Coop, i quali portano avanti delle nobili iniziative di solidarietà e di aiuto nei confronti dei paesi del Terzo mondo ignorando le condizioni lavorative dei dipendenti. (CORIS MON2001_04)

(11) Kodak mantiene comunque invariato il proprio impegno nella produzione e nella commercializzazione delle pellicole $35 \mathrm{~mm}$ di alta qualità [...]. (CORIS MON2001_04)

(12) La Kodak ha annunciato 10 mila licenziamenti, per tenere testa alla rivale nipponica Fuji. (CORIS STAMPASupplementi)

(13) Sony non è riuscita a opporsi all'ascesa della Apple con i suo iPod, così come la Playstation ha perso quasi tutto il suo smalto rispetto a Nintendo Wii o Microsoft xBox. (CORIS MON2008_10) 
(14) La Sony fu la prima a realizzare l'Hdtv: nel 1986, in collaborazione con l'Nhk (la tivù di Stato nipponica), presentò il sistema Muse con scansione a 1125 linee/60 quadri al secondo. (CoLFIS, periodico)

Capita inoltre che forme articolate e non-articolate si alternino nello stesso testo:

(15) La Migros rifiutava la riparazione in garanzia. [...] Interpellata da Spendere Meglio, Migros non ha preso posizione. (SM 6/05)

(16) Ford presenta il conto al nuovo governo. [...] Esistono vie d'uscita dalle paludi della recessione? Una la propone appunto la Ford, con l'idea dell'alleggerimento fiscale. (CoLFIS, periodico)

(17) Le reazioni delle due case non si sono fatte attendere: la Fiat, come al solito, ha smentito, Renault si è trincerata dietro un "no comment". (CoLFIS, quotidiano)

In queste proposizioni i predicati verbali istituiscono un contesto ristretto per il soggetto che legittimano (cf. La Fauci 2009: 62): i soggetti di preferire, annunciare, proporre, prendere posizione, smentire sono caratterizzati dal tratto [+ umano]. Si tratta di marchionimi che si possono descrivere come nomi d'impresa, per utilizzare un'etichetta referenziale approssimativa (in molti casi è peraltro pensabile che si possa trattare anche di nomi di marche); a questi usi sottostà un processo di personificazione o di metonimia (cf. La Fauci 2009: 196), per cui a smentire, in (17), non è "l'azienda Fiat" ma le persone che sono a capo dell'azienda o vi fungono da portavoce.

I soggetti di questi costrutti sono persone giuridiche; "umani collettivi", come osserva Grass (2002: 228) proprio in relazione alla combinazione con predicati "normalmente riservati agli umani". In questi casi la presenza o assenza di un determinatore - che generalmente, in questo tipo di costrutti, è $l a^{11}$ - non comporta una variazione sostanziale del valore degli elementi e della proposizione nel suo complesso.

Si è già accennato alla caratterizzazione referenziale dei marchionimi, in questo tipo di usi, come nomi d'impresa. Si vedano, al

11 Compare il maschile con nomi commerciali che contengono nomi comuni maschili, per es. "le Crédit Foncier de France" (Grass 2002: 230) o, in italiano, "il Credito Varesino"(LaR). 
proposito, alcuni casi di co-referenza ${ }^{12}$ tra il nome commerciale e $\mathrm{i}$ nomi comuni impresa, industria, azienda:

(18) La Lines, industria tutta italiana, tipico esempio di self made man locale entra in joint venture con la Pampers, una delle aziende della multinazionale americana Procter \& Gamble. (LaR)

(19) è in svolgimento ad Hannover l'assemblea straordinaria della Continental un'assemblea importante per lo sviluppo di questa scalata che è stata proposta da Pirelli per unire nel settore dei pneumatici la forza dell'azienda italiana con quella dell'azienda tedesca e creare un superpolo (LIP ME 11)

(20) Il capo della scuderia e gli uomini della Goodyear ascoltano lo sfogo del campione. Senna va avanti, stavolta piantando lo sguardo su uno dei tecnici dell' $a$ zienda che produce pneumatici: "Non funziona", dice Senna. (CoLFIS)

(21) La crisi recessiva che ha colpito il Giappone è la più severa da vent'anni e ha intaccato i due settori portanti dell'economia del Paese, l'automobile e l'elettronica. Aziende celebri nel mondo, come la Toyota e la Nissan (auto), la Matsushita e la Sony (elettronica), hanno dovuto ridurre la produzione e visto calare i profitti. (CoLFIS)

(22) Di fatto c'è che da qualche stagione aziende come Stefanel, Benetton, Ratti hanno scelto la Laguna per annunci di accordi, campagne pubblicitarie, collezioni. (CoLFIS)

(23) Una delle prime aziende automobilistiche ad attivarsi sui [sic] problema è stata la Volkswagen, che nel 1990 organizzò un simposio con i suoi fornitori sul tema "Emissioni nell'abitacolo delle vetture". (CoLFIS)

In questi brani la co-referenza tra nome commerciale e nome comune (azienda, impresa ecc.) si manifesta in modi sintatticamente varî: il marchionimo è seguito da un'apposizione complessa (frase incidentale) che contiene tali nomi comuni (18); è ripreso anaforicamente da costrutti con azienda (19-20); aziende precede una serie di nomi commerciali

12 Per il concetto di co-referenza cf. Gross (1977: 116): "La notion de coréférence est une relation sémantique portant sur deux groupes nominaux d'un discours. Elle exprime l'identité des personnes, choses, ou situations qui sont décrites par ces groupes." Nel costrutto "Un homme est arrivé, il s'est assis", per es., la co-referenza è indicata dal pronome $i l$ in relazione con un homme. 
(aziende come..., 21-22); il marchionimo è il secondo membro di un costrutto copulare di tipo specificativo (23; cf. Panunzi 2010).

I determinatori in variazione libera riguardano anche altri nomi commerciali, come Barbie:

(24) Crisi alla Mattel, il colosso dei giocattoli che produce la Barbie. Harry Pearce, direttore finanziario dell'azienda, si è licenziato. (LaR)

(25) Le scrittrici italiane hanno conosciuto e amato la Barbie? Non Dacia Maraini, che ha giocato "con bambole diverse: carine, ma non signorinelle e non sexy". (LaR)

(26) Se vi sembra che Barbie stia per trasformarsi in una bambola-robot è perché sta per affrontare la battaglia della sua vita. Le sue rivali? Le Bratz: Yasmin, Cloe, Sasha e Jade, bambole dall'ombelico scoperto coperte di lustrini, pellicce e pesanti ombretti, mai visti al di fuori dello Studio 54. (CORIS MON2005_07)

(27) Ogni anno nel mondo si vendono Bratz per due miliardi di dollari [...]. Questa somma è di poco superiore alla metà di quello che incassa Barbie, ma mentre le Bratz salgono, Barbie sta calando (vendite lorde globali scese del 13 per cento nel 2005). In Inghilterra, Australia e Sudafrica, le Bratz hanno venduto più di Barbie, arrivando fino al 60 per cento del mercato. (CORIS MON2005_07)

Il nome della bambola prodotta da Mattel ricorre con e senza articolo in contesti comparabili (soggetto e oggetto diretto, al singolare). La ricorrenza come nome nudo è particolare per un nome che si vuole "di prodotto".

Negli esempi (26-27) si nota la differenza con Bratz, nome di una bambola in concorrenza con Barbie, che nei dati ricorre perlopiù con articolo. Rispetto a questo marchionimo, Barbie si pone come nome dal carattere spiccatamente propriale, passibile di ricorrere senza articolo quando non è modificato. ${ }^{13}$ Più che per la sua origine di ipocoristico (dal nome personale Barbara), ciò si può forse attribuire al fatto che Barbie si presenta come un'unica entità declinata in numerose variazioni (si vedano le varie estensioni predicative: (la) Barbie in carriera,

13 In alcuni brani questo avviene in maniera costante: "In quei giorni fu fatto un sondaggio (un altro) su 150 bambine americane fra i 5 e i 12 anni: qual è il vostro gioco preferito con Barbie? Metà facevano spesso finta che Barbie fosse mamma. Un anno fa arriva così sul mercato anche in Italia la signora Cuore, che sarebbe Barbie rispettabilmente maritata" (LaR). 
(la) Barbie rock star, (la) Barbie in abito da sera), mentre "le Bratz" si pongono come plurali, come un insieme di elementi eterogenei, ciascuno con il proprio nome: "Yasmin, Cloe, Sasha e Jade" (26).

Lalternanza libera di nomi commerciali determinati e non-determinati si ritrova anche quando gli stessi nomi per i quali si individua il valore di "impresa" ricoprono la funzione di oggetto diretto o indiretto:

(28) Tutti fieri di contribuire al Sanremo dell'austerity: il milione di euro per la Hunziker è un ricordo. "Con quello che abbiamo risparmiato, abbiamo comprato l'Alitalia» scherza Chiambretti. (CORIS MON2008_10)

(29) A dispetto della frenesia di dichiarazioni, colpi di scena, ricette miracolose e clamorosi fallimenti, lo stato di crisi perpetua ha costretto Alitalia in uno stato di immobilità. (CORIS MON2005_07)

(30) [Amedeo Manuzzi] Tira però un sospiro di sollievo per la sua agenzia: "Fortuna che ci eravamo rivolti all'Alitalia. Se avessimo scelto una compagnia minore ci avrebbero condannati tutti." (CORIS STAMPAQuotidiani)

(31) Resta però incandescente la questione Malpensa: se per il ministro Bersani (in un'intervista a L'espresso) «non si può chiedere ad Alitalia di salvare Malpensa», il patron di Air One Carlo Toto [...] rilancia: [...]. (CORIS MON2008_10)

Un aspetto specifico dei nomi commerciali in posizione postverbale riguarda la coincidenza formale (apparente) tra gli usi in funzione di oggetto diretto e quelli definibili come usi avverbiali, per uno studio approfondito dei quali si rimanda al §4.4. I costrutti Ugo fuma la Marlboro e Ugo fuma Marlboro non sono equivalenti; in questo caso l'articolo non è una variante libera, come in La Marlboro decide... rispetto a Marlboro decide..., bensì manifesta un valore diverso del costrutto: ne fa un evento puntuale, di contro a una consuetudine. Per non parlare dei casi in cui la variante con articolo non si dà ('volare l'Alitalia) e il nome ha funzione univocamente avverbiale. Si tratta quindi di distinguere i casi in cui un determinatore non c'è, ma la sua presenza non cambierebbe il valore globale del costrutto (variazione libera), da quelli in cui esso non c'è e non potrebbe esserci senza che ciò muti il valore del costrutto (usi avverbiali del nome commerciale rispetto a normali costrutti transitivi, con marchionimo in funzione di oggetto diretto). 
Negli esempi che seguono si riconosce ai marchionimi un valore avverbiale:

(32) Roberto Benigni [...] È pallido, elegante, vestito di nero (ma sì, anche lui veste Armani come il suo amico Jarmush, ma non si deve sapere: deve sembrare solo un povero diavolo con un vestito troppo grande). (LaR)

(33) Vagli a spiegare che l'italiano medio veste Timberland o Levi's, mangia sempre più volentieri McDonald's o simili, guarda Dinasty e Dallas, sogna New York e California. (LaR)

(34) Nei momenti di riposo, però, le scarpe devono essere solo Diadora. La questione crea non pochi problemi. Molti giocatori sono infatti legati per contratto ad altre fabbriche: Altobelli calza Adidas, Conti è "proprietà" Puma, Bagni è pagato dalla Lotto. (LaR)

I predicati verbali che intervengono sono perlopiù quelli classici, che ricorrono negli slogan pubblicitari sin dagli anni Trenta del Novecento, in particolare vestire. Mangiare McDonald's è forse più atipico ma si inserisce in un'enumerazione di costrutti paralleli.

L'assenza di determinatori non è da considerare in maniera assoluta, bensì in una prospettiva di relazioni paradigmatiche e sintagmatiche. Si prenda l'esempio che segue, con predicato calzare, e lo si confronti con "Altobelli calza Adidas" (34):

(35) Helmut Newton [...] Porta dei jeans e una felpa, calza Reebock [sic] che sembrano nuove - ma sono così pulite solo perché così pulita è Montecarlo e così bianca di moquette è la sua casa al diciannovesimo piano di una torre sul mare-, sfoggia favolosi calzini rossi. (LaR)

Il nome Reebok è modificato da una subordinata relativa e pertanto non ha valore avverbiale ma nominale. Inoltre la determinazione zero, in questo caso, è funzionalmente equivalente a un articolo indeterminativo plurale (il cosiddetto partitivo): calza delle Reebok.

Non è un caso isolato. In molti esempi l'assenza di articolo davanti al nome commerciale non è indice di un valore avverbiale ma si presenta come alternativa all'indeterminatezza manifestata attraverso gli articoli (indeterminativi, partitivi, sia singolari che plurali):

(36) I ragazzi della Fiat [...] Indossano giubbotti di jeans, calzano Nike e portano lo zainetto "Invicta". (LaR) 
Basti ricordare il noto commercial della Pepsi in cui la scolaresca del futuro in gita sulla terra da un altro pianeta si guarda un po' intorno e scopre i resti della civiltà del secolo XX. Mentre tutti sorseggiano Pepsi, qualcuno raccoglie una vecchia bottiglia di Coca Cola, la porge alla professoressa per sapere cosa diavolo sia quell'oggetto e lei, dopo averla scrutata a fondo, dice perplessa: "Non ne ho idea...". (CORIS MISCVolumi)

(38) Ci limitavamo a vagabondare, sobbalzando lungo la costa, fermandoci per passeggiare, fare compere, escursioni, tracannare Guinness o ammirare semplicemente l'oceano. (CORIS MON2005_07)

A questi nomi commerciali si riconosce funzione di oggetto diretto.

Si delinea così un altro tipo di variazione libera oltre a quello riscontrato con i soggetti [ + umani] (e altre funzioni nucleari): esso riguarda marchionimi in funzione di oggetto diretto con valore di plurali (o) indeterminati. L'alternanza si pone qui non tra la e $\varnothing$ ma tra $\varnothing$ e articolo indeterminativo. Mentre l'equivalenza tra articolo determinativo e $\varnothing$ si potrebbe definire propriale, in questo secondo tipo di costrutti l'assenza di determinatore equivale piuttosto all'indeterminatezza, ciò che avvicinerebbe i marchionimi coinvolti ai nomi comuni. Ma è in gioco un intero costrutto, non un nome isolato; che peraltro può ricorrere identico in costrutti simili, per es. preceduto da articolo, senza per questo avere lo stesso valore in entrambi.

(39) Le britanniche Guinness e Grand Metropolitan hanno infatti fatto pace con il gruppo francese di beni di lusso, Louis Vuitton $\mathrm{MH}$, spianando così la via alla mega-fusione che farà nascere il colosso dei superalcolici. In base all'accordo annunciato ieri - Guinness pagherà a Lvmh 250 milioni di sterline. (LaR)

In questo costrutto, Guinness pagherà..., l'assenza di determinatori non ha lo stesso valore riscontrato nel brano (38), tracannare Guinness, dato che in (39) può alternare con i determinatori la o una (cf. §1.1.1, es. 21: "una Guinness ormai estesa in tutti i settori").

\subsubsection{Nomi commerciali sistematicamente determinati}

Oltre ai casi analizzati nel paragrafo precedente, in cui la determinazione non rappresenta un criterio di variazione funzionale, vengono in evidenza usi nominali per i quali invece la presenza di un determinatore rappresenta la forma non-marcata: 
(1) Ho un figlio di quasi sedici anni. Non gli posso comprare le Adidas o lo zainetto Invicta. (LaR)

La presenza dell'articolo davanti al marchionimo Adidas non è legata a contraintes sintattiche ma al contempo non pare essere oggetto di variazione libera: non c'è alternanza con "Non gli posso comprare Adidas" (cf. invece un ipotetico "Nike non può comprare (la) Adidas"). Adidas, in questo caso, non è (solo) una marca o un'azienda; conoscenze di tipo enciclopedico, condivise da larga parte dei parlanti italiano, permettono di attribuire al nome un referente specifico, dell'ambito delle calzature sportive. Il fatto che il determinatore sia al femminile plurale è qui rilevante: sostituendovi articoli maschili o singolari si otterrebbero costrutti non equivalenti per significato e più inconsueti.

Di seguito si riportano altri esempi:

(2) Per lanciare la Barchetta gli uomini di marketing della Fiat hanno scelto un colore deciso, un arancione acceso e forse non avevano tutti i torti. L'anima di questa spider è davvero calda. (CORIS STAMPASupplementi)

(3) Racconta che la stanza da letto della loro casa di Bogotà è rimasta come cristallizzata dal giorno in cui la fondatrice del partito "Oxigeno Verde" è uscita per non tornare più: i jeans su una sedia, il Rolex sul comodino. (CORIS MON2005_07)

(4) Capannelli di giovani attorno ai quali circolavano quantità spropositate di alcolici. Chi offriva un Tavernello, chi faceva girare un bottiglione di vinaccio dei Castelli. (CORIS STAMPAQuotidiani)

(5) Lui era filato in bagno a cambiarsi la fruit of the loom sudata con una polo color albicocca più che rispettabile [...]. (Brizzi 1994: 130)

La letteratura è solita distinguere tra questi nomi e quelli visti in precedenza identificandoli rispettivamente come "nomi di prodotto" e "nomi d'impresa". Uno dei criteri discriminanti è proprio quello della determinazione: in tedesco e francese, scrive Grass (2002: 229), “contrairement aux noms d'entreprises qui ne prennent que très rarement un déterminant, les noms d'appellations commerciales qui désignent le produit sont quasiment toujours assortis du déterminant". Vaxelaire 
(2005: 346), addirittura, reputa obbligatorio l'articolo in inglese e francese. Si aggiungono poi argomenti semantici e referenziali: dal punto di vista referenziale, in usi quali quelli esemplificati in (1-5) il nome commerciale non identifica più un'entità unica ma una grande quantità di oggetti simili o uguali; semanticamente il nome acquista un contenuto, non è più solo mera etichetta. Venendo a mancare i presupposti fondamentali del nome proprio, questo tipo di marchionimo è perciò classificato spesso come nome comune.

L'uso indifferenziato della categoria "nome di prodotto", così com'è proposta nella letteratura, è insoddisfacente e problematico. In questo modo, infatti, proprietà morfosintattiche, semantiche e referenziali sono attribuite ai singoli nomi, in maniera statica, disconoscendo i processi che intervengono dinamicamente nell'uso effettivo dei parlanti; salvo poi ricorrere al contesto quando si tratta di differenziare, in un esempio canonico, Renault come azienda e la Renault come vettura. Ma come si può affermare che in una Honda si ha a che fare con un nome di prodotto (cf. Vaxelaire 2005: 348) quando a essere considerato non è un nome, bensì un nesso nominale composto di determinatore + nome $\mathrm{o}$, meglio ancora, un nome correlato $\mathrm{a}$ un insieme di proprietà combinatorie, che lo determinano nella sua specificità e in opposizione ad altre?

Un secondo punto critico è che la letteratura (per es. Fèvre-Pernet \& Roché 2005) presenta la categoria "nome di prodotto" come una classe grammaticale sostanzialmente omogenea, opposta a quella di "nome d'impresa", ma non considera il fatto che tra i "nomi di prodotto" si annoverano marchionimi diversi dal profilo combinatorio (oltreché legale): nomi dati a prodotti specifici (la Vespa per un modello di scooter), nomi di una linea di prodotti (applicati eventualmente a un prodotto specifico: uno Scottex), nomi di marchio applicati a una serie di prodotti ( $i$ Pampers), nomi di un'azienda o di un marchio riferiti a un loro prodotto (tipicamente nell'ambito di veicoli, abbigliamento, elettronica ecc.: la Mercedes, le Doc Marten's, il Samsung). ${ }^{14}$ La funzione del determinatore è diversa nei vari casi, se si pensa in particolare che

14 Inoltre non sempre è possibile distinguere $\mathrm{i}$ vari tipi: può darsi che il nome dato a un prodotto singolo, per es. Nivea per la crema nata nel 1911, si estenda in seguito a una gamma di prodotti e persino a un'azienda, per cui oggi la Nivea ha almeno due referenti, come la Mercedes. 
nell'ultimo esempio essa distingue due valori: il Samsung (uso metonimico) e la Samsung.

Inoltre le due classi, nomi d'impresa e nomi di prodotto, sono stabilite perlopiù a priori, a partire da riflessioni astratte e che coinvolgono aspetti referenziali (o addirittura legati al marketing), supportate da test linguistici condotti sulla base di esempi inventati, come avviene in Fèvre-Pernet \& Roché (2005). Secondo questi autori, diverse categorie di marchionimi hanno come effetto diverse proprietà combinatorie, mentre in questa sede si sostiene il contrario: l'ipotesi, cioè, che siano le combinazioni - la sintassi - a far scaturire valori diversi dei nomi commerciali. La differenza può parere sottile ma comporta invece un paradigma di analisi linguistica profondamente diverso. Una conseguenza di non poco conto è che questo metodo permette di non passare sotto silenzio i molteplici usi dei nomi commerciali che esulano da quelli (nominali) solitamente considerati nella letteratura.

Invece di affermare che un nome è, a seconda dei contesti, nome di prodotto (quindi comune) o nome d'impresa/marca (quindi proprio), conviene studiare in quali costrutti la determinazione, intesa come categoria formale, è pertinente; in quali casi la correlazione tra marchionimo e determinatore fa scaturire un valore specifico, relativamente stabile. Si tratta di studiare il processo alla base di queste espressioni, un processo produttivo, disponibile agli utenti della lingua, per comprendere il quale è d'uopo non porre il problema in termini di categorie grammaticali: "Nella lingua, non di categorie si tratta, ma di funzioni e di interdipendenze processualmente determinate" (La Fauci 2011: 209).

Innanzitutto si nota che il panorama dei determinatori, quanto a genere e numero, è più variato rispetto agli esempi visti nel $§ 1.1 .2$, in cui i nomi commerciali sono preceduti, in genere, da la. Vi sono femminili singolari e plurali, maschili singolari e plurali, articoli determinativi e indeterminativi, numerali:

(6) Ci piacevano le moto e l'Harley Davidson era il nostro sogno, specie l'883, il modello meno costoso... "appena" 17 milioni. (CoLFIS, periodico)

(7) Un aspetto sconcertante del parcheggiare una Harley Davidson sono le inevitabili domande degli ammiratori. (LaR) 
(8) [...] a Monza tre Guzzi arrivarono ai primi tre posti e la stampa specializzata gridava allo scandalo per l'eccessiva supremazia tecnica delle moto italiane che vincevano tutto quello che c'era da vincere. $(\mathrm{LaR})$

(9) Siamo arrivati al punto che il tifoso non vuole stare in pace, ma in guerra. Se uno va ad allenarsi con la Ferrari ti prendono per un arricchito che si vende le partite, se ci vai in Cinquecento per uno che nasconde la Ferrari nel garage. Non c'è scampo. (LaR)

(10) Al Circuito di Vercelli, Nisotti, piazzandosi al secondo posto, precede di poco Nuccio Bertone, non ancora famoso come titolare di quella prestigiosa carrozzeria che ha "vestito" le Ferrari, le Maserati, le Alfa Romeo, le Lamborghini e le Fiat. (CORIS MISCVolumi)

(11) Se una volta bastava indossare l'eskimo per qualificarsi politicamente, oggi basta un Moncler per qualificarsi economicamente. Perché il Moncler è il più caro dei giubbotti come le Timberland sono le più care delle scarpe. (LaR)

(12) La sinistra, extra e ufficiale, portava l'eskimo verde e le Clark, i fascisti vestivano elegante, avevano le scarpe a punta e mettevano $i$ Ray-ban. (LaR)

(13) Ma dovendo acquistare un'auto usata, da chi la comprerebbe? Da Novelli o da Cardetti? Da Fassino o da La Ganga? "Mi comprerei una Vespa. [...]”. (LaR)

Lo stesso marchionimo può accompagnarsi a determinatori diversi, che ne manifestano la variazione di numero o di definitezza: l'Harley Davidson vs. una Harley Davidson (6-7), la Ferrari vs. le Ferrari (9-10).

\subsubsection{Ellissi o nominalizzazione? Il significato come processo}

In alcuni dei brani citati nel paragrafo precedente si osserva un nome comune in rapporto di co-referenza con il nome commerciale: "Ci piacevano le moto e l'Harley Davidson era il nostro sogno" (6); "il Moncler è il più caro dei giubbotti come le Timberland sono le più care delle scarpe" (11). Il genere di tali nomi comuni coincide con quello dell'articolo che precede il marchionimo (come lo è anche il numero, nei casi pertinenti: i Ray-ban per "gli occhiali", in 12, e non il Rayban). È un fenomeno che, nell'ambito dei nomi cosiddetti di prodotto, si presenta con notevole regolarità. Vi è chi, di conseguenza, interpreta la determinazione come riferimento implicito alla classe di oggetti cui il 
nome allude, per cui i costrutti Det Nmc sarebbero il risultato di un'ellissi. Si dice la Heineken perché si pensa la birra: così argomentano alcuni studiosi, descrivendo l'articolo, nelle sue fattezze categoriali, come rimando a un nome generico (Vaxelaire 2005: 349) oppure come rimasuglio di un nome comune che è stato cancellato dal sintagma in quanto ridondante.

Quest'ultima è l'opzione proposta da Gross (1977). Lo studioso interpreta i numerosi casi di nomi propri (non solo commerciali) utilizzati come nomi comuni, ossia preceduti da articolo, come risultato dell'ellissi di un "classificatore" ${ }^{15}$ il quale rende conto del genere e del numero di questi nomi propri (Gross 1977: 145). Il genere manifestato dall'articolo non sarebbe dunque arbitrario, bensì andrebbe ricondotto a quello del classificatore cancellato, per es. ville con i nomi di città (Gross 1977: 144-145). Per questo motivo, "lorsque l'on commande de la bière dans un café par un nom de marque, la forme sera toujours au féminin: Une (Kronenbourg + Heineken + Munich + Stella + etc.)!"' Gross continua: "Il semble naturel d'analyser ces formes par effacement du $N$ = bière ou de bière de la marque, ou de bière du nom de à droite de une" (Gross 1977: 145). Similmente argomentano Voigt (1989: 169) e Grass (2002: 230), per i quali il genere dei marchionimi dipende (perlopiù) dal nome generico corrispondente; in tedesco si avrà quindi das Erdinger perché Bier è di genere neutro.

Va però fatto un appunto alle osservazioni di Gross: "Il semble naturel..."? Certamente in alcuni casi l'argomento del nome (generico) "sottostante" non fa una piega; sotto Heineken sarebbe difficile, nei casi citati, leggere qualcosa di diverso rispetto a birra. Ma che dire di $i$ Birkenstock/le Birkenstock, che ricorrono grossomodo in egual misura (v. infra, §1.2.1)? Ancora, genere maschile e numero plurale di i Levi's saranno da ricondurre a "pantaloni" oppure a "jeans"? Qual è il nome generico dietro (Fiat) Barchetta: "coupé", "spider" o "auto", che

15 Nella sua trattazione, Gross (1977: 146) spezza una lancia a favore dell'ellissi, esecrata dalla grammatica generativa in quanto problematica per il trattamento automatico delle lingue. Però non si capisce in che misura la soluzione proposta, le cancellazioni, sia da preferire a una visione che chiami in causa le "interpretazioni” (Gross 1977: 146): anche nel caso delle ellissi si tratta in fondo di una ricostruzione. 
ricorrono tutti in combinazione con questo marchionimo? Ma, soprattutto, ha senso chiederselo?

Il ricorso a un classificatore o nome generico può forse spiegare, peraltro sempre ipoteticamente, il genere e numero manifestati dall'articolo; ma non spiega il processo che sta dietro questi usi. Inoltre non è chiaro se con "cancellazione" si intenda un'evoluzione diacronica o un processo sincronico. ${ }^{16} \mathrm{E}$ perché non potrebbe trattarsi, come sostiene Migliorini (1927: 93-94), di una "creazione metonimica" istantanea più che di un passaggio (logico o diacronico) da un costrutto a un altro?

È pur vero che esempi come il seguente sembrano avvalorare l'ipotesi dell'ellissi:

(1) Grande iniziativa Motta Fuji in occasione delle feste: basterà acquistare un Pandoro Motta insieme a un Panettone o a un altro Pandoro (tutti in confezione da un $\mathrm{kg}$ ) e compilare una cartolina allegata per avere in omaggio una piccola macchina fotografica Fuji. E se acquistate un terzo prodotto di questa serie avrete una Fuji con il flash. (CoLFIS, periodico)

Vi ricorrono infatti tanto il costrutto "completo" una piccola macchina fotografica Fuji quanto quello "ridotto" una Fuji con il flash, in rapporto di co-referenza con il primo. Una Fuji (con il flash) appare come la riduzione di una (piccola) macchina fotografica Fuji, fatta la tara della parte sentita come ridondante nel contesto specifico.

D'altra parte proprio il fatto che si tratta di una ripresa a distanza ravvicinata, e di un caso di co-referenza testuale, riduce l'importanza dell'esempio per avvalorare l'ipotesi di un'ellissi. Non avviene diversamente, infatti, nei casi di ripresa con valore pronominale dell'articolo: "gli allievi bravi e i meno bravi", dove il modificatore - "meno bravi" funziona come nome. Con i nomi commerciali, inoltre, spesso il co-referente generico non è reperibile nel contesto (cf. le Clark e $i$ Ray-ban,

16 In una direzione simile si muove l'appunto critico di Migliorini (1927: 94): “Un'espressione è ellittica solo se considerata rispetto all'uso linguistico di un certo parlante in un certo momento (o tutt'al più, con qualche approssimazione, rispetto all'uso medio di un gruppo linguisticamente compatto a cui il parlante appartenga): se questo s'ignora, di ellissi non si potrebbe propriamente parlare." Secondo lo studioso è indispensabile non ritenere l'ellissi "una specie di Nemesi cieca, [...] non illudersi d'aver spiegato il fenomeno parlando di «ellissi che colpisce un certo gruppo di parole» o simili” (Migliorini 1927: 93-94). 
supra, es. 12); anzi, con nomi come Vespa, attribuiti a un prodotto specifico, questo è il caso più frequente. D'altro canto, di che cosa sarebbe un'ellissi, Vespa, dal momento che difficilmente si sentirà o leggerà $l o$ scooter Vespa o simili? Resta dunque da chiarire come si possa descrivere il processo alla base di questi costrutti e che li accomuna nelle loro diverse fattispecie.

La questione è più ampia rispetto a quanto scrive Gross: il genere del marchionimo (e dell'articolo che lo precede) è, infine, solo un accidente, un prodotto di questo processo, ma non trova necessariamente la sua ragion d'essere in un nome generico cancellato. Certamente è possibile spiegare il genere ricostruendo un nome in rapporto con il valore manifestato dal marchionimo; d'altronde, l'italiano ha due generi ed è pertanto quasi inevitabile che, di fronte a un nesso Det $N m c$, non si faticherà a trovare un nome del genere corrispondente: una Jacuzzi? vasca con idromassaggio; un Mac? computer; e via dicendo. Ma questo, in primo luogo, non spiega i casi in cui l'alternanza tra determinatore maschile e femminile (la Jacuzzi/il Jacuzzi) non manifesta un valore differente del nome (come invece per il Samsung/la Samsung). In secondo luogo, non rende conto del fatto che i parlanti, quando usano questi costrutti, fanno a meno di nomi comuni generici ogniqualvolta essi non siano pertinenti nel discorso; e non lo sono quasi mai, pare, per nomi come Vespa. Perché e secondo quali criteri il linguista dovrebbe pertanto ricostruirli?

Alla questione del genere espresso dal determinatore si aggiunge quella della significazione, tanto più scottante in quanto i nomi coinvolti sono, o si ritengono essere, nomi propri: in questi casi, combinati a un particolare determinatore, essi implicano infatti uno specifico contenuto semantico di volta in volta chiaro ai parlanti, benché a volte esso possa essere delineato solo approssimativamente (per es. 'scarpe', 'moto', 'macchina fotografica'). In questa sede si ritiene che il significato dei nomi commerciali sia non un presupposto degli usi "come nomi comuni", bensì il risultato di un processo, che si può descrivere nei termini di una nominalizzazione.

(2) Il pH non determina la qualità dell'acqua, ma solo il gusto. Per cui, se vi piacciono i sapori aciduli potete scegliere la Uliveto o la Fiuggi. Se preferite un'acqua meno decisa, ci sono le alcaline come la Vera o la Panna. (CORIS STAMPAPeriodici) 
Il brano (2) ha per tema le acque in bottiglia, al proposito delle quali si legge: "ci sono le alcaline". L'aggettivo alcalino, che ricorre preceduto da articolo, si nominalizza. Ricorrono poi la Vera, la Panna, più sopra la Uliveto, la Fiuggi: nomi di acque minerali, o meglio attributi, predicazioni ("l'acqua X", "l'acqua di marca X"), usati come nomi.

Gli esempi che seguono illustrano due casi di aggettivi in funzione nominale per i quali ha avuto luogo una specificazione del significato: il numerale settemmezzo, riferito a un tipo di motocicletta, e l'aggettivo qualificativo utilitaria per un tipo di automobile.

(3) La progressiva risposta delle sospensioni, unita alla intuitività della guida, ed a vibrazioni abbastanza contenute (tranne qualche picco ai medio-alti), fanno della nuova settemmezzo Honda una macchina di gran comfort, da godersi in souplesse nelle gite fuoriporta [...]. (CoLFIS, periodico)

(4) Successo anche per la guida-virtuale della Punto, allestita all'esterno del Salone. Qui, chiunque può sedersi nell'utilitaria Fiat e provare l'ebbrezza di guidarla rimanendo fermo. (CoLFIS, quotidiano)

Non c'è attributo che non possa essere nominalizzato; anche il nome commerciale, dunque. Si tratta di una trasformazione funzionale, che non lo modifica dal punto di vista morfologico; d'altro canto, già di nome si tratta. Funzionalmente, però, il marchionimo - come gli altri nomi propri - partecipa della natura attributiva propria degli aggettivi (cf. il "predicato di denominazione" di Kleiber 1981): l'aggettivo bianco predica, di qualcosa, che è "di colore bianco"; Ugo predica di qualcuno il "chiamarsi Ugo" e Barilla, si potrebbe aggiungere a questo punto, il "chiamarsi Barilla" o, meglio, 1' "essere di marca Barilla".

Si sta qui affermando che, in una prospettiva funzionale, i nomi commerciali non sono poi così diversi dagli aggettivi. L'idea non è nuova negli studi del settore. Vaxelaire (2005: 217-218) vi dedica alcune pagine; sulla base del costrutto *This oil is Shell confuta però l'opinione di alcuni studiosi (perlopiù non linguisti) che ritengono che i nomi di prodotto siano aggettivi. L'asterisco posto da Vaxelaire, peraltro, è presto smentito dai dati (v. §4.3.2). Senza andare lontano, basta un 
noto slogan per illustrare la coincidenza funzionale di nome commerciale e aggettivo: "Liscia, gassata o Ferrarelle?". ${ }^{17}$

Una manifesta funzione predicativa del nome commerciale si riconosce non solo nei casi in cui esso segue un nome comune (l'acqua Panna, le scarpe Nike; v. cap. 2):

(5) La differenziazione tra il sito Fiat e quello Alfa si manifesta anche nel fatto che da una parte il sito non offre molte informazioni sull'azienda, la sezione "Fiat per la scuola" è invece un viaggio all'interno del codice della strada e della sicurezza nel viaggiare [...]. (CORIS MISCRiviste)

(6) Ma tra i sogni e i divertimenti c'è lo stesso scarto qualitativo che separa le tagliatelle della nonna da quelle Barilla. (CORIS MISCVolumi)

(7) [...] altri calendari illustri, da quello della sartoria Battistoni che vanta amici famosi che posano da modelli, da Paolo Bulgari a Gigi Proietti, a quello Lavazza con angeli e diavoli. $(\mathrm{LaR})^{18}$

In questi casi, al posto del nome commerciale nel nesso quello $\mathrm{Nmc}$ non potrebbe stare se non un attributo; lo si vede in particolare nell'es. (6), in cui a "le tagliatelle della nonna" sono contrapposte "quelle [scil. le tagliatelle] Barilla". ${ }^{19}$

Pure negli esempi seguenti i marchionimi hanno valore di predicazione rispetto a un nome comune, esplicito in (8) - "apparecchi che non siano Apple" -, implicito negli altri:

17 Probabilmente l'ipotesi che considera il nome commerciale un aggettivo dal punto di vista funzionale non ha nulla a che vedere con il fatto che negli Stati Uniti i nomi di marca e prodotto sono concepiti e utilizzati come aggettivi (rispetto ai produttori francesi, che li ritengono nomi propri), come sostiene Vaxelaire (2005: 218); forse, anzi, la politica linguistico-normativa delle aziende americane è rivelatrice di un aspetto linguistico che le forme superficiali - unite a secoli di pregiudizi categoriali - contribuiscono a oscurare.

18 Va distinto dall'es. seguente: "[la] concorrente numero uno della Segafredo, ossia quella Lavazza che da anni ambienta in Paradiso gli spot sul caffe”"(LaR). Qui il deittico non ha funzione pronominale, bensì aggettivale.

19 Questo costrutto compare di frequente con nomi propri prototipici, in particolare patronimici. Alcuni esempi dal corpus LaR: "Il decreto Visentini e quello Berlusconi"; "Vi sono tanti piani: quello Hussein, quello Reagan"; "il nodo Taranto e quello Genova". Non mancano, nello stesso corpus, esempi con nomi commerciali: "lo scoglio da superare sembra proprio quello Fiat"; "le [azioni] Peugeot $[\ldots]$ vantano un rapporto fra prezzo e utili più interessante di quello Fiat". 
(8) Il rapporto fra Linus e il suo iPhone è di amore e odio. Perché hai comprato il melafonino? «Per tre motivi. Primo, perché sono consapevole di essere un fighetto di 50 anni, e come tale devo comportarmi. Poi per i rigidi comandamenti tecnologici dettati da mia moglie, architetto, che sul lavoro non concepisce apparecchi che non siano Apple. Infine, molto semplicemente, perché è bello». (CORIS MON2008_10)

(9) Ho due figli fanatici della fotografia [...]. Vorrei regalare loro un buon apparecchio fotografico, magari Canon o Nikon, ma girando per la città e guardando le vetrine dei vari negozi ho scoperto che ci sono enormi differenze di prezzo. (CORIS STAMPASupplementi)

(10) L'unica a non tramontare mai è la polo, meglio se Lacoste. (CoLFIS, quotidiano)

Sono costrutti che, in ossequio all'ipotesi esposta in precedenza, si possono parafrasare come predicazioni denominative ridotte: "la polo, meglio se è di marca Lacoste".

(11) Dalla Svizzera è partita la richiesta di accertamenti basati su registrazione di trasmissioni radio e rapporto dei piloti. Quello Alitalia - che sostava sulla tax way «Bravo Bravo» usata per gli scavalcamenti nell'ordine delle partenze avrebbe domandato più volte l'autorizzazione al decollo, sempre negata, alla torre di controllo. (CORIS MON2001_04)

In (11) il marchionimo Alitalia implica una predicazione metalinguistica di secondo livello: esso modifica un nome comune [+ umano], pilota, per cui la predicazione non sarà "di marca X" ma piuttosto "(pilota) di un aereo della compagnia chiamata Alitalia". La funzione attributiva è peraltro la stessa dei casi precedenti.

La natura attributiva dei nomi commerciali, che predicano l'“essere di una determinata marca", si associa alla possibilità di farne un uso nominalizzato:

(12) Giorgia arriva all'appuntamento. [...] Lui sfodera un completo Ferrè estate con cravatta Ermenegildo Zegna. È cosparso di Armani uomo. (CORIS NARRAT Racconti)

Nell'esempio (12) Armani uomo - un marchionimo dalla natura intrinsecamente attributiva, si potrebbe dire, in quanto non identifica un prodotto specifico ma si applica a collezioni prêt-à-porter, gioielli, borse ecc. - non modifica nessun nome comune e solo il contesto, in 
particolare il predicato cui si combina, permette di comprendere il riferimento a un profumo.

Il caso è particolare (si tratta di un testo di narrativa) ma non del tutto anomalo. Lo si può prendere come esempio estremo dei nomi commerciali che ricorrono, nel contesto, come predicati di denominazione divenuti argomenti, dal momento che l'argomento di cui sono predicati è noto o facilmente deducibile dal contesto.

La Toyota, $i$ Wrangler, la Nivea e così via ricorrono quindi come nomi; ma sono processi, non enti, come del resto tutti i nomi propri (cf. La Fauci 2011: 209; 218-219). I nomi commerciali sono "etichette", attributi, non solo nelle strutture in cui modificano un altro nome ma anche negli usi propriali (Fiat decide), che rappresentano due faccette dello stesso processo: Fiat è "l'azienda chiamata Fiat" ma non è necessario esplicitarlo. È peraltro possibile far precedere il nome dall'articolo la, come per altri versi nel caso dei nomi propri personali (la Giulia, il Carlo). In questi casi l'articolo non manifesta il nome, non ne è un rimasuglio; l'articolo è il nome (chiaramente non in senso categoriale), ossia l'argomento di cui il nome proprio (di marca) è il predicato e che ne manifesta genere e numero.

Per spiegare strutture come le Adidas, dunque, non è necessario fare ricorso all'ellissi e ricostruire un nome comune soggiacente, ciò che può avvenire in maniera più o meno intuitiva ma resta, nella maggior parte dei casi, un'ipotesi non verificabile. Qui si propone invece di concepire il nome commerciale Adidas come predicato, in rapporto predicativo rispetto all'articolo medesimo. Di volta in volta sarà possibile riconoscere un operatore che attua il processo di nominalizzazione: "scarpe di marca X", "occhiali di marca X", "auto di marca X"; ma non è necessario stabilirlo con esattezza. In questi casi, l'utente della lingua non esplicita il tipo di prodotto - e si può pertanto ritenere che esso non sia rilevante -, la sua marca invece sì. E lo è, naturalmente, anche il determinatore, nei casi non-marcati: la determinazione, correlata alla nominalizzazione, viene in luce come "la specificazione argomentale di una predicazione nominale" (La Fauci 2009: 146).

Il processo di nominalizzazione appena descritto costituisce una relazione tra un luogo comune e la sua designazione, delineandosi quindi come processo metalinguistico (cf. La Fauci 2011: 217). Nell'enunciare 
le Nike, la Mercedes, la Vespa non conta più che il marchionimo sia il nome di una marca, di un'azienda o di un prodotto: un "atto di battesimo" istituisce, al contempo e in maniera correlativa, un luogo comune e il suo nome. Rispetto a queste espressioni, i vari sandali Birkenstock e vettura Fiat hanno carattere patente, manifesto, ma vige, in questi casi, una simile relazione con il luogo comune, che si perfeziona negli usi argomentali i Birkenstock, la Fiat. Si tratta di una predicazione di tipo nominale, metalinguistico, che in quanto tale è paragonabile a quella dell'antonomasia classica: la tigre di Cremona come denominazione di un'entità (personale, individuale) che assurge a luogo comune, a conoscenza condivisa da una comunità. Similmente avviene con i costrutti qui considerati. ${ }^{20}$ D'altro canto, quali nomi propri più dei nomi commerciali sono soggetti a un fenomeno tipicamente umano e sociale qual è la moda, basata sull'istituzione e la condivisione di luoghi comuni?

(13) E per chi vuole tenersi a sinistra, [c'è] l'eterno Birkenstock ormai declinato in decine di versioni (quest'anno, ci vuole il modello infradito trattenuto dal cinghietto dietro la caviglia nei colori base, nero, bianco e testa di moro o nelle versioni a fiori) [...]. (LaR online ${ }^{21}$ )

Non sono qui necessari nomi comuni, per es. sandalo, per delineare il valore di "l'eterno Birkenstock". Il nome commerciale stesso svolge funzione di nome comune: perché costituisce un luogo comune, non tanto perché sia categorialmente o morfosintatticamente attribuibile a tale classe. Si tratta di un uso, come scrive Grass (2002: 224), correlato alla notorietà del prodotto ma, diversamente da quanto afferma lo studioso, non necessariamente legato al valore connotazionale dei prodotti designati: mentre per Grass "on achète du ciment, pas du Lafarge" (Grass 2002: 224), in verità non è difficile pensare a situazioni e gruppi sociali (o professionali: per es. dei muratori) in cui si utilizza

20 Così, per es., di fronte alla proposizione "Auto e moto erano la sua grande passione, qualche tempo fa il padre gli aveva comprato una Ford" (CoLFIS, quotidiano), conoscenze condivise permettono di stabilire che il nome commerciale Ford va riferito, come specificazione marchionimica, al precedente "Auto" (e non a "moto").

$21<$ ricerca.repubblica.it/repubblica/archivio/repubblica/2009/07/02/dallo-stival etto-ai-leggings-ecco-gli-imperdibili.html?ref=search $>$ (18.2.2015). 
"il Lafarge" per riferirsi a un cemento in particolare, in opposizione magari a quello di altre marche. ${ }^{22}$

Quanto affermato sinora, in particolare l'invito ad abbandonare l'ipotesi dell'ellissi per spiegare il processo alla base della struttura le Adidas, non toglie peraltro che sia possibile stabilire equivalenze tra costrutti in cui i nomi commerciali hanno funzione argomentale e altri in cui ricoprono funzione predicativa rispetto a un nome comune, due usi accomunati da una coincidenza di valori:

(14) Gli occhiali Ray Ban da aviatore: [costano] cinquantamila a Washington, a Roma almeno il doppio. (CoLFIS, quotidiano)

Gli occhiali Ray Ban corrisponde a i Ray-ban dell'es. (12) nel paragrafo precedente. Ma ciò non comporta che si debba chiamare in causa una cancellazione per illustrare il fenomeno; è sufficiente, infatti, descriverlo in termini di pertinenza e di esplicitezza.

(15) Lo chef degli chef, ovvero Gianfranco Vissani, è un grande uomo di cucina oltre che grande conoscitore di bottiglie. Di vino, certo. Ma pure di acque minerali. Dal suo regno gastronomico a Civitella del Lago, Baschi, ci ha aperto la sua carta ideale delle bollicine. Cominciamo dall'antipasto: quale acqua abbinerebbe? "Ottima è la San Faustino, interessantissima, molto particolare. È leggera e diuretica [...]". Passiamo ai primi. "Uso molto la Gaudianello [...]. Per le zuppe di orzo e fagioli è d'obbligo la San Benedetto, rocciosa come le Alpi da cui proviene. [...]". Siamo arrivati ai secondi. "Col pesce ci sta bene la Nepi che ha corpo e un'effervescenza delicata [...] la cacciagione si affianca bene a una Perier [sic], molto forte. Le carni bianche vanno d'accordo con le acque salate. La Badoit o la Vittel, per esempio, sono perfette con il pollo. Per le carni dolci non c'è di meglio della San Pellegrino, salata e garbata insieme [...]". Anche i contorni prevedono una loro acqua? "Certo: verdure cotte e crude trovano l'accordo migliore con la Ferrarelle che è gentile e non stufa mai [...]. I soufflé, invece, vanno di rigore con la Panna, che è leggerissima". Veniamo ai formaggi? "Per quelli cremosi consiglio l'inglese Hilton, che è salatina". E siamo alla frutta, e ai dolci. "Ottima una Levissima che ripulisce e ha la funzione digestiva del sorbetto [...] torte, crostate, cremini e gelati si sposano bene con $l a$

22 In generale, l'azienda stessa non gradisce l'uso argomentale del nome d'impresa per un suo prodotto, com'è invece comune nella lingua quotidiana (v. Parte I, §3.3). In un inserto pubblicitario sullo yogurt Yomo (LIP MD 18) il marchionimo Yomo entra sempre a far parte di costrutti attributivi ("yogurt della Yomo", quattro volte) e solo una volta è usato come nome, ma prontamente corretto: "vogliamo dare uno sguardo a tutti questi vari Yomo questi vari yogurt". 
Sangemini, di sapore appunto dolciastro". Dimentichiamo qualcosa? "I superalcolici: per accompagnarli ci vuole l'Evian [...]”. (CORIS STAMPAPeriodici)

Nel brano (15) ricorrono quattordici marche di acque in bottiglia, tutte al femminile (dove il genere è manifesto), combinate con articoli sia determinativi che indeterminativi; marchionimi, quindi, utilizzati nominalmente. Acqua ricorre alcune volte ed è il topic del testo: in questo senso non stupisce che l'unico elemento di variazione, che determina una pertinenza, sia il nome di marca delle varie acque, al quale si accompagna una predicazione volta a dichiararne le caratteristiche. D'altro canto, molti dei marchionimi contenuti nel brano ricorrono comunemente anche in testi che non parlino esplicitamente di acqua, dal momento che il valore specifico correlato a la Ferrarelle o la Vittel è diventato ormai una conoscenza condivisa dalla comunità linguistica.

Altre volte, invece, capita che la pertinenza sia da riferire al contesto specifico:

(16) Un mondo a parte è quello delle agende in pelle "griffate". Hanno prezzi alti e qualità: la più cara è quella col marchio d'oltremanica Burberry's che nell'unica versione in pelle martellata sfiora le trecentomila. La Timberland, ora che ha gli importatori diretti, costa un po' meno rispetto all'anno passato (240 mila lire). (LaR)

La Timberland è da mettere in relazione con la frase che precede, in cui è questione di "agende in pelle", e non allude all'azienda omonima. L'articolo la costituisce qui una sorta di ripresa pronominale: La Timberland come "quella [agenda] con il marchio Timberland".

Avviene pure che le predicazioni del marchionimo si nominalizzino, in contesti specifici, senza che ricorra un argomento manifesto, quale il determinatore. Nella rivista per consumatori Spendere Meglio ciò avviene di frequente, dato che numerosi articoli ruotano attorno a un unico tipo di prodotto di cui sono valutate diverse varianti di marca $\mathrm{e}$ produttore (come in 15); negli esempi che seguono si tratta di salviette umide (17) e forni a vapore (18):

(17) Le salviettine M-Budget di Migros e quelle di Denner hanno raggiunto un risultato sufficiente perché sono meno resistenti e morbide. [...] Un risultato ben diverso per gli altri prodotti esaminati. Prix Garantie, Soft e Camomille contengono formaldeide o liberatori di formaldeide. Prix Garantie, Soft Comfort (Migros), e Camomille (Coop) ottengono quindi un risultato insufficiente. (SM 5/07) 
(18) Può anche succedere che si debba riempire il serbatoio dell'acqua durante la cottura. Con Miele, Miostar e Weber è risultato abbastanza scomodo perché bisogna prima di tutto aprire il portello del forno. [...] Hoover perde acqua quando si toglie il serbatoio [...]. Alcuni apparecchi sono un po' rumorosi durante il funzionamento. Il più silenzioso è Weber. I più rumorosi, per contro, sono Standard Sr, Hoover e Panasonic. (SM 3/10)

Nei brani ricorrono, in funzione argomentale, marchionimi di vario tipo: nomi di una gamma di prodotti (Prix Garantie), di un prodotto specifico (Camomille, che qui identifica un prodotto anche se certamente non è marchio registrato), nomi d'impresa (da Miele a Weber). Com'è ovvio, in un altro contesto gli stessi nomi, utilizzati sempre in funzione nominale (e senza articolo), possono avere un altro referente, per es. un aspirapolvere:

(19) La rivista per i consumatori Saldo ha mandato in laboratorio dieci modelli di aspirapolvere tra i più venduti [...]. Solo tre modelli, sui dieci sottoposti al test, sono in grado di eliminare bene la polvere dal tappeto: Miele, Hoover e Electrolux. Un risultato mediocre in questo criterio è andato a Philips, Miostar, Trisa e Bosch; insufficiente per Satrap, Rotel e Dyson. (SM 6/10)

Qui è determinante il tema attorno cui ruota l'articolo della rivista, in funzione del quale il solo nome commerciale risulta pertinente. Ma non è detto che l'utilizzo nominale di questi marchionimi sopravviva alla menzione estemporanea del giornale. In numerosi altri casi invece - se ne sono già citati alcuni - la combinazione $\operatorname{Det} \mathrm{Nmc}$ ha un valore relativamente stabile, indipendente dal contesto. Non si tratta di un significato reperibile nei dizionari, bensì di un uso regolato da istanze sociali, di una competenza condivisa da gruppi sociali più o meno vasti; un significato mutevole, com'è caratteristico dei nomi propri rispetto a quelli comuni. ${ }^{23}$

Approfondiamo ora la questione del determinatore. In genere nella struttura Det Nmc ora delineata non si riscontra variazione libera tra le Birkenstock e Birkenstock, in contrapposizione all'alternanza

Ci si potrebbe sbizzarrire a indagare le competenze enciclopediche di un parlante italiano in merito a questi usi dei nomi commerciali, alla stregua di quanto è stato fatto per i marchionimi passati a generici (cf. Pöcksteiner 2008): chi usa (ancora) il Sony? Quali cereali si conoscono (ovvero: si nominano attraverso un marchionimo) oltre a $i$ Kellogg's? La giacca di piume è ancora un Moncler e, se di marca Gil Bret, può essere un Gil Bret oppure resterà nell'anonimato? 
esistente tra Birkenstock e la Birkenstock (\$1.1.2). Quest'osservazione ha portato studiosi come Vaxelaire (2005: 346) a parlare di un articolo "obbligatorio" con i nomi commerciali utilizzati in riferimento a un prodotto (Van Langendonck 2007a: 238 è meno radicale) e che entrano a far parte di un'enciclopedia condivisa, per es. le Nike, la Coca-Cola, la Porsche. I dati spingono a riconsiderare quest'affermazione: vi sono infatti alcune tipologie di contesti che favoriscono l'assenza di determinatore.

(20) Pochissimi gli stilisti che comprano cose non griffate da loro. Eccezioni: Dolce e Gabbana a volte acquistano da Fiorucci; Armani sfoggia spesso Nike bianche; Ferré ama i foulard, di cui ha una collezione, e gli impermeabili di Hermès. (CORIS STAMPAPeriodici)

(21) Un malavitoso di Cittadella viene ora ricercato per gli incendi: è lui che ha appiccato due volte il fuoco, mandando distrutte Porsche e Bmw. (CoLFIS, quotidiano)

In (20), "Armani sfoggia spesso Nike bianche", il valore del marchionimo - femminile, plurale: si veda l'aggettivo che lo segue - si differenzia dal caso in cui genere e numero dello stesso non sono specificati (né specificabili), per es. in "Armani veste Nike". Nel brano citato Nike è un nome, non usato avverbialmente, e comporta un riferimento specifico a un prodotto; l'assenza di determinatore si deve al valore di plurale indeterminato. ${ }^{24}$ Lo stesso si può affermare riguardo a (21).

Un altro contesto che condiziona l'assenza di determinatore è l'elenco:

(22) Quando alle 13.10 il bandito, sui 25 anni, biondiccio, statura media, Ray-Ban scuri e abbigliamento sportivo, entra nella banca ci sono i dipendenti, un cliente e la guardia forestale Amedeo Silvestri [...]. (CoLFIS, quotidiano)

Nel prosieguo del brano citato in (22), in combinazione con la preposizione con, il marchionimo ricorre preceduto dall'articolo: "Il biondino con i Ray-Ban non continua la fuga ma torna sui suoi passi".

Possono ricorrere senza determinazione i nomi commerciali che entrano in elenchi o costrutti bimembri preceduti da una preposizione:

24 In francese, per es., lo stesso costrutto riporterebbe des Nike, con articolo indeterminativo plurale. 
(23) I commentatori radio e tv sperimentano un sistema di appunti che dovrebbe farla finita con le schede-atleti messe a punto in mesi di lavoro con pennarello e coccoina. (LaR)

(24) I ragazzi della Microsoft, che vivono a pane, Coca-Cola e software, lo considerano un tipo solitario. (CORIS STAMPAPeriodici)

(25) A un tratto, al vecchio Alex era venuta in mente una specie di sequenza cinematografica che gli aveva dato il capogiro: una delle due ragazze, tornata in Italia, che raccontava a un gruppo di sette otto amiche tutte uguali, con giacca jeans, pantacalze su ginocchio valgo, assorbente esterno e superga in tela blu, di quanto s'era divertita in Inghilterra con Maddalena. (Brizzi 1994: 134)

La preposizione in è spesso seguita da un marchionimo non determinato:

(26) “[...] Giorni fa ho viaggiato in Harley Davidson con mia moglie Corinna da Las Vegas al Colorado". (CORIS MON2001_04)

(27) Già negli anni 50 la rivista Life aveva pubblicato in copertina il presidente Eisenhower in Lacoste, ma le magliette sono diventate davvero globali solo nel $1963[\ldots]$. (LaR)

(28) Se Altan fosse più "furbo" avrebbe già sostituito il suo operaio con uno scattante signor Rossi in Timberland, Lacoste e cartella da executive. (LaR)

Questo avviene in particolare in riferimento a veicoli (in Harley-Davidson), capi d'abbigliamento (in Lacoste) o scarpe (in Timberland); per una trattazione specifica si rimanda ai $\$ 3.4 .1$ e 3.4.2.

Si consideri ora la preposizione $d i+$ nome commerciale non determinato:

(29) E il messaggio è ben diverso da quello del tranquillo e un po' rassegnato Manfredi, che nel caffè trova consolazione e, in qualche modo, relax. Al contrario, nella tazzina di Lavazza bevuta all'estero si troverà, fanno capire i pubblicitari, la sferzata necessaria a lavorare di più $[\ldots]$. (LaR)

(30) Qualcuno si ricorda i televisori marca Zenith, fabbricati nell'Illinois? Furono sommersi, trent'anni fa, dalla marea giapponese, da milioni e milioni di Sony. $(\mathrm{LaR})$

(31) Anche a Raffaella la Vespa di Pallina era passata inosservata. Le madri si accorgono di tutto ma non capiscono niente di motorini o di Sony. (Moccia 2004: 194) 
In questi casi la preposizione è indispensabile al costrutto, diversamente dalla struttura jeans di Missoni, in cui non è necessaria (v. §2.2.1).

In conclusione, l'affermazione di Vaxelaire va modulata in questi termini: il determinatore si incontrerà regolarmente in contesti non-marcati, mentre può mancare in altri - quelli appena delineati - senza che ne consegua un diverso valore del nome commerciale.

Con i nomi propri prototipici l'assenza di determinazione equivale alla determinatezza, come ricorda Gross (1977: 147): “employés sans Dét, les noms propres doivent être considérés comme définis, puisque leur pronominalisation donne lieu aux pronoms appelés traditionnellement définis". Lo stesso si può dire degli usi non-marcati di nomi commerciali con valore di azienda. Negli esempi visti or ora, invece, la casistica è più complessa: nel caso di elenchi e plurali (e con la preposizione $d i$ ) è probabile si tratti di un valore indeterminativo (perlopiù plurale, o nome di massa), mentre con le preposizioni si propenderà in genere per un valore determinativo, singolare o plurale a seconda dei casi: in Harley-Davidson equivarrà a "con l'Harley-Davidson", in Clark's a "con le Clark's". In ogni caso, il valore resta distinto da quello, individuato in precedenza, relativo all'azienda: (la) Nmc. È possibile che anche questo tipo sia preceduto dalla preposizione in, per es. nel nesso lavorare in Fiat ( $\$ 3.4 .1$ ); questa struttura, però, ha un equivalente nel tipo alla Fiat, ciò che non vale per i casi qui individuati.

\subsubsection{Riflessioni sistemiche sulla determinazione}

Le pagine che precedono hanno indagato sulla determinazione dei nomi commerciali approfondendone non solo l'aspetto fenomenico (di che tipo è, quale genere manifesta ecc.) ma anche, in un'ottica sistemica, le relazioni di equivalenza tra strutture determinate e non-determinate, al fine di comprendere in quali casi i determinatori (o la loro assenza) sono motivati sintatticamente, in quali l'alternanza tra presenza e assenza non influisce sul valore globale del costrutto e così via. Nello spirito di Saussure (1916: 142), infatti, le cose valgono non tanto per ciò che 
sono, quanto per ciò che non sono ma che potrebbero essere (cf. La Fauci 2011: 81-82). Stabilire il valore dell'articolo e di altri determinatori è un passo necessario per delineare il valore del nome commerciale $\mathrm{e}$, in ultima istanza, il suo significato.

Dall'analisi emerge una sostanziale novità: per l'italiano, presenza, rispettivamente assenza di determinatori non rappresentano un criterio che permette di distinguere univocamente tra nomi di prodotto e nomi d'impresa. In maniera più affidabile si può invece stabilire se la determinazione costituisca una variante libera. Si delineano in questo modo usi che avvicinano i nomi commerciali al valore di "impresa", nel caso vi sia appunto variazione libera, e di "prodotto", nel caso questa non si dia (con la particolarità degli usi avverbiali, distinguibili dagli usi come oggetto diretto anche in funzione della non-equivalenza con il nesso determinato). Paradossalmente, quindi, i nomi cosiddetti d'impresa (la Galbani chiude) sono individuabili come tali perché possono ricorrere preceduti da articolo, mentre i nomi cosiddetti di prodotto sono il più delle volte determinati (una Lacoste rossa), ma in dati contesti, non rari, la ricorrenza senza determinatore sembra l'unica plausibile (in Lacoste).

Quanto alla presenza del determinatore, è possibile a volte osservare un notevole divario in funzione dell'enunciatore: la ditta produttrice (proprietaria, dal profilo giuridico, del nome commerciale in questione) o altri. Nel primo caso si riscontrano usi tendenzialmente non determinati, che mettono in rilievo l'aspetto propriale del nome: le imprese, infatti, vogliono stabilire e conservare il carattere di status del proprio nome o del nome dei propri prodotti contro eventuali abusi e degradazioni (cf. Vaxelaire 2005: 350).

Con i nomi riferiti a un'impresa la tendenza va oggi nella direzione di un uso non determinato, perlomeno in testi scritti, per cui l'articolo determinativo sembra essere una variante diacronicamente marcata. Diversamente da quanto annotava Renzi nel 1988, oramai non sono più solo gli impiegati della Fiat a dire "Lavoro in Fiat" invece che "alla Fiat" (Renzi 1988: 396). ${ }^{25}$ Sono in primis le aziende stesse a porsi

25 Per uno studio approfondito sarebbe necessaria un'analisi quantitativa su dati comparabili di epoche diverse; in questa sede vi si rinuncia, data l'impostazione sincronica del lavoro. 
come non-determinate, o meglio, come intrinsecamente determinate; per accertarsene basta dare un'occhiata alle pagine internet di Barilla o di Alfa Romeo e contare gli usi articolati del nome commerciale, se pure ve ne sono.

Al riguardo, il commercio al dettaglio svizzero presenta un panorama particolare (cf. Janner 2012a: 163-168). Se si prendono le due principali concorrenti, Migros e Coop, si osserva che, a una situazione sostanzialmente omogenea quanto agli usi dei parlanti (nei dati ricorrono sia Migros e Coop che la Migros e la Coop), si contrappone un'attitudine linguistica diversa da parte delle imprese: sul proprio sito Coop si presenta rigorosamente senza articolo, mentre la pagina di Migros presenta tale nome regolarmente corredato di articolo determinativo. Le imprese attuano due strategie comunicative diverse: Coop si pone come nome proprio, a carattere tendenzialmente personale ("fare acquisti da Coop"), rinunciando ad aggettivi possessivi che implicherebbero la presenza correlata di un determinatore (v. §1.1.1), mentre Migros si rappresenta come un luogo comune, un'antonomasia, sottolineando la vicinanza ai clienti con l'uso di possessivi ("alla vostra Migros"). La variazione libera acquista dunque risvolti stilistici.

La comunicazione d'impresa sfrutta la determinazione - o meglio la sua assenza - per affermare il carattere propriale di un nome, ovvero la sua unicità e personalità. L'assenza d'articolo con i nomi commerciali è generale strategia di allontanamento dal nome comune, verso l'acquisizione di quel carattere esclusivo (cf. Vaxelaire 2005: 350) che si accorda bene con il motto "turn a brand into a religion" (Fowler \& Iwatani Kane 2011).

Questo vale per i nomi relativi a un'impresa ma anche, e a maggior ragione, per quelli riferiti a un prodotto, che non presentano variazione libera quanto alla determinazione: il caso, riportato da Vaxelaire (2005: 350), di "versez Maïzena", come si legge sul pacchetto del prodotto, è marcato rispetto all'espressione determinata comunemente in uso (“de la Maïzena”). Per portare un esempio autoctono, Vespa ricorre regolarmente senza articolo sul sito italiano: 
(1) Da oltre 50 anni Vespa muove intere generazioni sui modelli pensati per i più giovani. Tra i più significativi di questa lunga storia c'è Vespa Primavera, lanciata nel 1968. (<it.vespa.com $>26$ )

L'uso non determinato trova la sua ragion d'essere nel tentativo dell'impresa di restituire carattere propriale a un marchio registrato che già pochi anni dopo la sua introduzione, complice anche l'omonimia con il nome comune, veniva declinato al plurale (in un racconto di Gadda del 1954 si legge "vespe e lambrette"; cf. GDLI s.v. Vespa $a^{5}$ ). In tempi recenti vespa è stato utilizzato anche per veicoli prodotti da altre case, subendo un allargamento di significato potenzialmente rischioso dal punto di vista legale (cf. Parte I, §3.3).

Oltre all'assenza marcata di determinatori, la strategia di riproprializzazione prevede l'uso rigoroso di maiuscola e plurale invariabile:

(2) La nuova Vespa Sprint raccoglie l'eredità delle Vespa più veloci e giovanili [...]. $\left(<\right.$ it.vespa.com $>^{27}$ )

Strategie linguistiche comparabili sono messe in atto per un altro prodotto emblema della creatività italiana, Nutella, quando l'enunciatore è riconducibile all'azienda stessa:

(3) Per esempio, per ottenere il gusto inconfondibile di Nutella, usiamo una miscela di cacao davvero speciale: la nostra. (pubblicità Ferrero, 2016 ${ }^{28}$ )

Un marchionimo come Alfa 159 non corre certo il rischio di subire genericizzazione, eppure in uno slogan pubblicitario ricorre anch'esso senza determinatore:

(4) Se cerchi una scusa per scegliere Alfa 159, preparati a trovarne molte. (pubblicità, 2007)

$26<$ www.it.vespa.com/it/index.html\#/vespa/IT/it/Modelli/Vespa-Primavera $>$ (27.2.2015).

$27<$ www.it.vespa.com/it/index.html\#/vespa/IT/it/Modelli/Vespa-Sprint> (27.2.2015). Gli articoli sono in questo caso determinati dal concomitante ricorrere di modificazioni attributive (cf. supra, §1.1.1).

28 Da LaRepubblica, novembre 2016. L'uso esclusivo del marchionimo non determinato si incontra pure sul sito $<$ www.nutella.com $>$ (5.1.2017). 
L'assenza marcata è da attribuire a una strategia di personificazione del nome commerciale, che si avvicina così al nome proprio prototipico, l'antroponimo. Lo si vede in opposizione al brano seguente:

(5) Cosa dice Opel al riguardo? Il portavoce Christoph Bleile [...] aggiunge in tono scherzoso: «A noi non interessa se si opta per la versione a benzina o a gasolio: l'importante è che si scelga un'Astra». (SM 4/2007)

Rispetto al banale costrutto transitivo scegliere un'Astra, scegliere Alfa 159 si presenta non solo come uno stile di vita, complice la posizione postverbale (cf. §4.4), ma come una scelta esistenziale, come "scegliere Maria (piuttosto che Laura)". Chi sceglie Alfa, si potrebbe dire, è più alfista di chi, semplicemente, compra un'Alfa (cf. Janner 2012a: 171).

\subsection{Genere e numero}

I tratti di numero e genere dei nomi commerciali, che nelle strutture Det Nmc sono manifestati perlopiù dal determinatore, sono analizzati non tanto in funzione della relazione con un nome comune soggiacente quanto nei termini di una pertinenza sistemica. Ciò vale in particolare per il genere: alcune alternanze di maschile e femminile sono funzionali alla distinzione di diversi valori del nome commerciale, altre non lo sono.

\subsubsection{Genere}

Gli usi dei nomi commerciali che sono definibili in relazione a un luogo comune (cf. §1.1.4) si caratterizzano per una certa regolarità tanto nel numero quanto (soprattutto) nel genere, manifestati nell'articolo e in altri determinatori o modificatori: le Adidas, Nike bianche ecc. A ogni costrutto si associa perlopiù un solo genere.

Si dà però anche il caso di uno stesso nome commerciale che presenta, in contesti comparabili, genere maschile o femminile, senza 
che sia possibile individuare una variazione quanto al valore globale del costrutto:

(1) E comunque, io non sarei così intransigente nel giudicare il "look" del Papa: molto meglio le Prada rosse che le Birkenstock (per chi non le conoscesse, trattasi dei famosi ed orribili sandali tedeschi) che tutti i miei colleghi tedeschi quotidianamente calzano al lavoro. (LaR online ${ }^{29}$ )

(2) Munitevi invece di un biglietto per gli Usa o le Eolie, in tasca la "Profezia di Celestino", in cuffia De Gregori, ai piedi $i$ Birkenstock, e quando siete al dunque dimostrate di apprezzare una vasca idromassaggio, di possedere un telefonino e un fax, di saper passare con disinvoltura dalle amenità del giardinaggio al linguaggio di Internet. (LaR)

(3) Immerso nei vapori della Jacuzzi, cullato dall'idromassaggio, il boss Ciro Formicola contemplava il golfo di Napoli dalla finestra del bagno. (LaR)

(4) Come fa una donna così ampia ad entrare nel bagno ad imitazione delle celebri modelle di Bonnard? Si è fatta costruire uno Jacuzzi su misura? (LaR)

(5) Due nibbi in divisa da poliziotto su una Guzzi sfrecciano da via Chiaia a via S. Anna di Palazzo [...]. (LaR)

(6) Ho dato un pessimo esempio ai miei figli, pensando invece di onorare la memoria di un padre motociclista fanatico che mi portava neonata sul Guzzi e mi insegnò ad amare il più straordinario antidepressivo che esista: la velocità. (LaR)

L'alternanza di genere, nei casi di Birkenstock (1-2), Jacuzzi (3-4) e Guzzi (5-6) qui esemplificati, non è significativa; è considerabile alla stregua di una variazione libera. ${ }^{30}$

Marchionimi come (un) Ford Transit ricorrono perlopiù al maschile, ma vi è pure un esempio al femminile (9):

(7) Sanguinante, stordito, Cirillo viene caricato su un furgone, un Ford Transit. $(\mathrm{LaR})$

$29<$ www.repubblica.it/2005/b/rubriche/letterealdirettore/figli-lupa/figli-lupa. html? ref=search $>$ (18.2.2015).

30 Un fenomeno che si osserva non di rado con forestierismi: il/la (e-)mail, il/la bandana. 
(8) Tutto è cominciato in via Madama Cristina quando un ladro si è impossessato di un Ford Transit bianco che il proprietario aveva lasciato incustodito e aperto. (CoLFIS, quotidiano)

(9) Dorino Stoyka, un falegname rumeno di 30 anni che aiutava Giancarlo ad installare gli infissi della nuova casa, andando in cerca dell'ex assistente di volo che si era allontanato con alcuni amici a lui sconosciuti, si imbatte in una Ford Transit di colore azzurro. (LaR)

Nel corpus LaR il marchionimo Moncler preceduto da articolo, con valore di prodotto, è sempre maschile, tranne nel brano seguente:

(10) Gli ospiti del petroliere che ha acquisito fortuna e titoli nobiliari appartengono a un mondo che non vuole essere avvicinato dai cronisti. [...] Portano grandi decorazioni sullo sparato della camicia, indossano lo smoking con la disinvoltura con cui un paninaro porta la Moncler. (LaR)

Il fenomeno dell'equivalenza tra nomi che presentano generi diversi non sembra riguardare un grande numero di nomi commerciali; d'altro canto è importante rilevare non tanto l'effettivo numero di ricorrenze, quanto l'esistenza di una tale possibilità nella lingua.

In altri casi il genere si correla invece alla differenziazione di valori:

(11) $[\ldots]$ due architetti trentenni, i più teneri di tutti, arrivati su una Yamaha, ex Pantera, lui ha il cranio rasato e una maglia che dice 'solo gli elefanti portano l'avorio', lei ha l'ombelico in vista, c'è attaccato un anello. (LaR)

(12) Bernhardt nel suo romanzo Il soccombente mostra Gould chiuso nella stanza che manipola il suo pianoforte per ottenere il suono che desidera. Corrisponde alla verità? Gould prediligeva certi strumenti, aveva prima un vecchio Steinway, poi uno Yamaha, ma non so se li manipolasse, piuttosto lavorava molto con il montaggio. (LaR)

Per il marchionimo Yamaha si identificano qui due valori diversi: una motocicletta, al femminile (11), e un pianoforte (in contesti particolari) per il maschile (12). ${ }^{31}$

31 Valori che ricorrono in strutture equivalenti del tipo $N$ Nmc: "Due giovani sono fermi a bordo di una moto Yamaha ad alcuni metri di distanza" (LaR); "Appena entrati, allo stand Ricordi, un pianoforte Yamaha ultima generazione dà il benvenuto suonando da solo (è programmato) melodie anni Sessanta" (LaR). 
Il marchionimo Peugeot alterna femminile e maschile in riferimento rispettivamente a un motorino (13-14) e a un' automobile (15-16):

(13) Alcuni ragazzi con dei Peugeot e degli SH 50 si fermano proprio davanti alla scalinata. Anche Raffaella arriva in quel momento. Trova un piccolo buco dall'altra parte della strada, di fronte al benzinaio prima della chiesa, e ci si infila con la sua Peugeot 205 quattro porte. (Moccia 2004: 98)

(14) Nonostante la logica sembra indicare che i mezzi più adatti al traffico sono proprio i piccoli e maneggevoli "cinquantini", come vengono chiamati i ciclomotori di cilindrata compresa nei $50 \mathrm{cc}$, i risultati di mercato hanno sancito la vittoria dei maxiscooter di grossa cilindrata, divenuti protagonisti di un trend in crescita esponenziale. Se si esclude, infatti, il Peugeot Speedfight $100 \mathrm{cc}$, e le due motociclette, Ducati 600 Monster ed Aprilia Pegaso, nei primi 20 posti della classifica relativa alle immatricolazioni dei primi 9 mesi di quest'anno, troviamo 17 scooter le cui motorizzazioni vanno da $125 \mathrm{cc}$ a $250 \mathrm{cc}$. (LaR)

(15) Nella sua memoria collettiva c'è tutta la Francia di questo secolo, il Fronte Popolare e il "maquis" dei ferrovieri, le illusioni dell'Union de la Gauche e il naufragio mitterrandiano, lo stalinismo e i robot, le vittorie di Pirro e le sconfitte napoleoniche di una classe operaia che sognava la Peugeot in garage e si ritrova con la minaccia della "minestra dei frati" per i nuovi poveri. (LaR)

L'opposizione di genere distingue quindi due valori diversi del nome commerciale Peugeot. ${ }^{32}$

Vi è poi una variazione comune a tutti i nomi commerciali utilizzati in riferimento tanto a un'azienda quanto a un prodotto: nel primo caso il nome appare come femminile singolare, nel secondo varia nei diversi contesti. Così i già noti le Timberland, i Levi's, il Moncler si oppongono a la Timberland, la Levi's, la Moncler. Qualora genere e numero del primo tipo (azienda) fossero coincidenti con quelli del secondo (prodotto) - la Yamaha, la Mercedes -, saranno altre caratteristiche combinatorie a differenziarli: il fatto che, in contesti non-marcati, solo il

32 Non è chiaro se valga lo stesso anche per Scottex, perlomeno dal punto di vista lessicografico. Il dizionario Zingarelli 2015 distingue il sostantivo femminile, per "nome commerciale di un tipo di carta soffice e assorbente confezionata in rotoli e usata spec. in cucina", e quello maschile, con il valore di "foglio di tale carta strappato da un rotolo". Per il dizionario italiano-francese Larousse, invece, scottex è "sostantivo femminile invariabile" (e corrisponde a un altro nome commerciale, sopalin); cf. <www.larousse.fr/dictionnaires/italien-franc ais/scottex/57103> (2.7.2015). 
secondo alterni con l'articolo indeterminativo senza che ciò ingeneri variazioni sostanziali di significato, o che nel primo tipo di costrutto l'articolo costituisca variante libera.

(16) La Sony inserirà il Blue ray in tutte le nuove Playstation 3 in arrivo a marzo. (CORIS MON2005_07)

(17) Rapido si spostò verso il margine e lì scese, si sgranchì le gambe e le braccia, respirò più volte profondamente. Poi si tolse il casco e fu come un tuono: aveva il sony incorporato. (CORIS NARRATRacconti)

(18) «Okay ragazzi, se vi perdona lui posso perdonarvi anch'io. Ovviamente, se vi venisse la tentazione di riprovarci sappiate che vi strapperò il cuore con un mignolo» gigioneggiai spavaldo «e soprattutto...», estrassi il Sony di tasca, «...ho registrato questa conversazione, quindi avete una bella spada di Damocle e una croce di Santandrea. [...]» (CORIS NARRATRomanzi)

(19) La chiama. Lei sembra non sentirlo. Quando le è vicino si accorge perché. Sta ascoltando il Sony. (Moccia 2004: 223)

(20) Sono le 19,30 di ieri sera. L'uomo che guarda fisso il piccolo Sony trinitron ha le lacrime agli occhi, appena nascoste dalle lenti spesse. (CORIS MON2005_07) $)^{33}$

Sony, al femminile (16), alterna con il Sony, riferito (in particolare nella narrativa di fine Novecento) a un apparecchio portatile per ascoltare musica (17-19) - un Walkman, per usare un altro marchionimo - ma anche a un televisore (20).

Il genere femminile (perlopiù plurale) ricorre inoltre in riferimento alle azioni dell'azienda: ${ }^{34}$

(21) Chiusura in ribasso per il listino britannico che ha finito con lo scontare la debolezza d'apertura di Wall street. [...] Tra i titoli guida, le Guinness sono calate

33 Si noti, en passant, che l'uso al femminile, in (16) e in altri casi qui non riportati, vede comparire il nome commerciale come soggetto, mentre l'uso al maschile ricorre, negli esempi citati, con funzione di oggetto diretto. Non sarà un caso, se si riflette al tratto [ \pm umano] (su questo v. $\$ 4.1$ ).

34 Come per il nome riferito all'azienda, anche per i titoli il genere può essere maschile se maschile è il nome comune contenuto nel nome commerciale: "si è visto un Credito Varesino, che più volte abbiamo indicato come esempio di ingiustificata sottovalutazione, fare un balzo del 9 per cento" (LaR). 
di 7,5 punti nonostante le notizie dell'accordo da 171 milioni di dollari con la statunitense Barton. (LaR)

(22) Le Fiat sono state al centro di scambi sostenuti e il titolo ha registrato un ultimo prezzo di 4.716 lire (1,79\% sul riferimento precedente). (CoLFIS, quotidiano)

(23) Ma vediamo il listino. Grande attività sulle Fiat, con oltre 13 milioni di azioni trattate, su Olivetti (18 milioni) e sulle Generali (circa 2 milioni). I titoli della casa automobilistica torinese hanno guadagnato l'1,69\% a quota 6.373 [...] Le Montedison hanno invece perso lo $0,15 \%$. Grande balzo, ma su un quantitativo molto limitato, per le Volkswagen, che hanno guadagnato il 20\%. (CoLFIS, quotidiano)

(24) Ma quello che conta è l'umore generale. E a questo proposito si può dire tranquillamente che la maggior parte degli operatori italiani e stranieri ha giocato la carta della continuità del governo pentapartitico poi ampiamente confermata dalle prime proiezioni Doxa del pomeriggio. Nella mattinata si è vista una Fiat superare di colpo le 3000 lire per portarsi fino a 3050 nell'immediato dopolistino. (LaR)

Grass (2002: 230-231) osserva che, in tedesco, in riferimento alle azioni il nome proprio d'impresa è utilizzato al plurale e senza determinatori (per lo studioso si tratta di un'ellissi di “NPR-Aktien"); la versione francese è al femminile singolare. Per l'italiano, stando agli esempi citati, la forma non-marcata è invece femminile plurale, con determinatore.

In tutti i casi visti sinora, il genere del marchionimo si manifesta unicamente nel determinatore. Con il nome commerciale Vespa, femminile, si riscontra invece una variazione di genere correlata a suffissi derivativi:

(25) Eravamo più belli. Forse più buoni. Comunque ci divertivamo con molto meno. C'era "Lascia o raddoppia", il vespone e la mitica 600. (CoLFIS, quotidiano)

(26) Vespino bianco già parcheggiato, Adelaide era proprio davanti alla Feltrinelli che guardava le copertine dei libri in vetrina con indosso un maglione verde e un sorriso zen imperscrutabile ma molto onnicomprensivo. (Brizzi 1994: 20)

Vespa è femminile, mentre vespone e vespino compaiono al maschile. Il genere è manifesto morfologicamente nel suffisso del marchionimo (e si confronti il caso di (la) donnal(il) donnone). Sono casi poco frequenti, 
essendo piuttosto rari gli adattamenti morfologici dei nomi commerciali (cf. cap. 5).

Nei brani che seguono si hanno esempi di non-pertinenza di genere e numero:

(27) Bloccato l'accordo fra la Lines e $i$ Pampers della Procter. L'ANTITRUST DI BRUXELLES BOCCIA L"EUROPANNOLINO”. ROMA - Leon Brittan ci ripensa e nega il suo consenso al matrimonio del pannolino, annunciato nel settembre '90 da una joint-venture fra $i$ Lines di Ancona e l'americana Procter \& Gamble, produttrice dei Pampers. (LaR [occhiello, titolo e articolo])

(28) Il pericolo era sorto tre anni fa quando, dopo anni di battaglie a colpi di nuovi prodotti e investimenti pubblicitari, la Lines di Pescara e $i$ Pampers prodotti dalla Procter \& Gamble avevano dato vita alla joint venture Fater [...]. La scelta su quale dei due prodotti cedere è caduta per forza di cose sulla Lines. Il marchio di Pescara infatti, pur vincendo in Italia il confronto con la Pampers, ha una struttura commerciale molto più semplice della Procter \& Gamble, presente in ben 150 Paesi. (LaR)

I due nomi commerciali Lines e Pampers ricorrono tanto al femminile singolare quanto al maschile plurale, in una maniera che sembra giocare sulla variatio più che rispondere a criteri di effettiva differenziazione: infatti è possibile solo in senso traslato che dei pannolini (i Lines in 27, $i$ Pampers in 28) diano vita a una joint venture.

Mercedes è un caso particolare. Il marchionimo ricorre al maschile con diversi valori:

(29) Ruggeri nei giorni scorsi ha acquistato un Mercedes del valore di 320 milioni da affiancare agli altri undici pullman rimasti in esercizio e risparmiati dal fuoco. $(\mathrm{LaR})$

(30) Il funzionario della Battistoli ha mantenuto la parola e si è presentato accompagnato solo da un dipendente, poi assieme a Righi si sono avviati verso Monteviale, una località a cinque chilometri da Vicenza dove il mattino aveva abbandonato il furgone blindato, un Mercedes bianco. (LaR)

(31) Auto incendiate a Pisa, polizia allertata e ancora croci ad Avellino: in serie $\mathrm{B}$ monta la tensione attorno alle squadre in difficoltà. In Toscana sono state bruciate le due macchine (un Mercedes e una Peugeot) del capitano del Pisa, Roberto Bosco. (LaR) 
(32) Gli affari vanno a gonfie vele e lui si entusiasma. Cambia una macchina al mese, sempre più cara (ma quando si compra il Mercedes 5000 è Sbardella a dirgli di scegliersi un'auto meno vistosa). (LaR)

(33) Saranno un'ottantina i nomadi di questo accampamento, slavi, serbi, bosniaci dell'etnia khorakanè, la più reietta, la più povera, anche se, come spesso avviene, non mancano, mischiati alla miseria, simboli e feticci del benessere: un telefono cellulare, un Mercedes. (LaR)

(34) Ci sono colpe storiche anche degli agricoltori. Truffe e dintorni. «Ma questa è povera gente, mica hanno la tv al plasma o il Mercedes. Non sanno neanche cosa vuol dire vacanze». (CORIS MON2008_10)

Nei primi due esempi (29-30) il genere maschile - ragionando in un'ottica di ripresa anaforica - pare giustificato dai nomi comuni pullman e furgone, dei quali è una specificazione (di tipo metalinguistico). Ma che dire dei successivi? I "feticci del benessere" di cui si parla nei brani successivi, in contrasto con situazioni di povertà, non saranno certo un autobus o un furgone. Se si ragiona in termini di ellissi sarà forse possibile trovare un nome generico maschile per motivare il genere di Mercedes; ma è più economico considerarlo nei termini di un'opposizione al comune la Mercedes. Tale opposizione distingue non due significati dello stesso marchionimo usato nominalmente, quanto due valori sociali: il Mercedes non è solo un'automobile, è uno status symbol. Così anche Pino Daniele, in Che soddisfazione (1991), canta "Volevo un Mercedes bianco". ${ }^{35}$ In alternativa (e combinatoriamente) si può pensare che, con l'aumentare della cilindrata, il genere maschile - in quanto opposto al femminile non-marcato, non in quanto maschile - sia più appropriato, sin dai tempi del classico slogan "Metti un tigre nel motore".

A conclusione di questo giro d'orizzonte sul genere, riprendendo le osservazioni di Kolde (1995: 403) a proposito dei nomi propri, si constata che anche con i nomi commerciali l'opposizione di genere

35 Citando il brano musicale, Minestroni (2010: 174n) commenta: "Come in Joplin, la Mercedes o «il Mercedes» partenopeo, diviene l'emblema di un lusso ludico". Sembrerebbe che la studiosa valuti il genere maschile come una variante diatopica; i dati (e un orecchio un po' attento) portano invece a propendere per un uso marcato dal punto di vista stilistico, eventualmente diafasico. 
permette di differenziare valori. ${ }^{36} \mathrm{Si}$ è vista in particolare la tendenza al femminile (singolare) per nomi riferibili all'impresa e al femminile (plurale) per le azioni, che si oppongono ai nomi riferiti a un prodotto dell'azienda. Ma più che tali distinzioni di tipo semantico è fondamentale il principio dell'opposizione sistemica. Esso infatti, in quanto individuabile in maniera discreta, è più efficace nello spiegare il panorama composito e variegato del genere dei nomi commerciali di quanto non lo sia il ricorso a un nome generico non effettivamente presente.

\subsubsection{Numero}

In relazione ai nomi commerciali la categoria grammaticale del numero condivide con quella del genere una caratteristica: in determinati contesti e con determinati valori, i nomi commerciali ricorrono regolarmente al plurale o al singolare, come mostrano gli articoli e/o fenomeni di accordo. I Levi's, le Superga, i Ray-Ban ricorrono perlopiù al plurale quando identificano prodotti specifici (ess. 1-3), mentre altri marchionimi - per es. Citroën, Marlboro, Rolex - si ritrovano tanto al singolare quanto al plurale (4-9):

(1) La Jeanseria del corso è l'unica che sconta i mitici Levis, un articolo stravenduto che nessuno si sente di ribassare [...]. (CORIS STAMPAQuotidiani)

(2) Allora, dice Rubini, tutti giocavano con le Superga. Erano generalmente bianconere. (LaR)

(3) [...] il colonnello Richard Fee Er [...] dirige con la determinazione e lo humour che l'emergenza continua richiede la complessa macchina organizzativa. I suoi fedelissimi, come il cap. Cado, hanno in comune $i$ Ray-Ban e un minimo fatalismo sull'instabilità cronica di quella che fu l'Africa Equatoriale Francese. (CORIS STAMPAQuotidiani)

(4) All'incrocio si è fermata una Citroën dalla quale sono scesi quattro Sudamericani [...]. (CoLFIS, quotidiano)

36 Gli esempi di Kolde (1995: 403) fanno riferimento alla variazione das schöne Deutschland (nome di nazione)/die schöne Deutschland (nome di battello). In italiano una situazione comparabile si ha per es. con il Lazio (nome di regione)/ la Lazio (nome di squadra sportiva). 
(5) Poi una breve corsa dalla sede ginevrina delle Comunità europee al palazzo delle Nazioni Unite. Scortate da decine di auto delle teste di cuoio svizzere le Mercedes, le Citroen e le Alfa Romeo dei ministri Cee sono accorse da Perez, appena giunto da Parigi con un Dumas cupo e taciturno. (LaR)

(6) Forse più che a Fellini, si può pensare a Tinto Brass. Specialmente quando accende la Marlboro in maniera sensuale: le telecamere zoomano freneticamente. (LaR)

(7) I birmani non fumano le Marlboro - è proibito importarle - ma con il loro tabacco si fanno da soli i loro cheroot; non portano blue jeans, ma i loro longyi. (CORIS MON2005_07)

(8) La faccia del militante socialista di base di Milano, è diversa dallo stereotipo che possiamo immaginare. Magari non porta i Ray Ban, non ha il Rolex al polso, ha la barba lunga ed è anche trasandato. (LaR)

(9) Ovunque il mercato dei Rolex è gestito da qui siccome - come ha dimostrato l'inchiesta del 2006 al Monte di Pietà - i clan napoletani, soprattutto quelli del centro storico riescono a immettere $i$ Rolex nuovamente nel circuito nazionale $\mathrm{e}$ internazionale di vendita. (CORIS MON2005_07)

Nondimeno, anche alcuni nomi commerciali che ricorrono al plurale con i valori descritti nel $\S 1.1 .3$ - possono comparire al singolare, come Superga, Levi's e Birkenstock:

(10) Della celebre Superga modello 2750 - che sarebbe la superclassica scarpa da tennis bassa e stringata vista ai piedi, per citare solo qualche nome, di Carolina di Monaco e di Maria Josè di Savoia, di Giorgio Armani e di papa Giovanni Paolo II - sono stati fino ad oggi venduti oltre 50 milioni di paia. (LaR)

(11) Le produzioni di Levi's, infatti, avvengono tutte in fabbriche del gruppo, in Europa e negli Usa, con aggiustamenti per ogni mercato nazionale: "La linea di base è quella che ha fatto famoso il Levi's nel mondo, gli adattamenti vengono incontro ai bisogni del cliente sui vari mercati. Un classico, ma flessibile”. $(\mathrm{LaR})$

(12) Nell'attuale stato di incertezza riguardo al futuro dei mercati, la familiarità di una Birkenstock rivestita di pelo ci dà sicurezza, per quanto possa apparire ridicola. (LaR online ${ }^{37}$ )

37 <ricerca.repubblica.it/repubblica/archivio/repubblica/2014/11/16/lanuovame diocrita42.html?ref=search $>$ (18.2.2015). 
Come il genere, il numero può manifestarsi nella flessione morfologica oltreché nel determinatore. Ciò avviene con marchionimi derivati da nome comune (Vespa) o percepiti come tali: aspirina, divenuto ormai nome generico; sofficino, nato al plurale - e usato al plurale dalla ditta produttrice - ma che ricorre anche al singolare nei dati analizzati (cf. inoltre Zardo 1997: 31):

(13) D'altronde, grazie alla sua meccanica semplice e affidabile e alla facilissima guida [...], la Vespa riusciva benissimo a sostituirsi a un'utilitaria. (LaR)

(14) [...] è nato a Roseto degli Abruzzi uno dei più moderni stabilimenti italiani per la produzione e l'assemblaggio di ciclomotori. A cominciare dal Velocifero, lo scooter dalle linee tondeggianti che da una parte strizza l'occhio alle Vespe e alle Lambrette del dopoguerra mentre dall'altra ricorre a piene mani agli ultimi ritrovati della tecnologia. $(\mathrm{LaR})^{38}$

(15) Già da alcuni anni circolavano rapporti secondo cui una persona che ha sofferto un infarto può ridurre il pericolo di un secondo attacco prendendo regolarmente l'aspirina; ma sino ad oggi non c'erano prove che gli effetti benefici potessero estendersi anche a chi non ha ancora dato segno di malanni cardiaci. (LaR)

(16) "Senza ricetta, possiamo vendere solo aspirine, analgesici, antinfiammatori blandi, i cosiddetti prodotti da banco. La gente ha tutte le ragioni per protestare". (CoLFIS, quotidiano)

(17) Ma non è solo il talk-show arrabbiato a inquinare le marche. Spesso, in trasmissioni come Forum, condotto da Rita Dalla Chiesa, arrivano ospiti specialisti in scenate. Che effetto fa allo spettatore vedere il sofficino che sorride zittito da una lite fra dirimpettai che si scagliano pantofole e anche topi? (LaR)

(18) Nei prossimi mesi gli spot a base di testimonial raddoppieranno. Maldini venderà le Nike, Teocoli-Faletti-Gnocchi cucineranno $i$ sofficini, un probabile Henry Kissinger offrirà fondi d'investimento e perfino l'algida, bellissima, Catherine Deneuve presterà la sua esile figura all'acqua di Fiuggi. (LaR)

38 Non sempre però Vespa e Lambretta sono pluralizzati anche morfologicamente: "Bianco. È il colore delle Vespa che vengono utilizzate per le esecuzioni" (CoLFIS, periodico); "Il lamierino della vecchia Italsider forgiava le nostre piccole Fiat, le Lambretta e le Vespa, le lavatrici e i frigoriferi dei signori Borghi e Zanussi”(LaR). 
Nelle strutture seguenti il numero è invece oggetto di variazione libera:

(19) A differenza di altri parchi divertimento a Legoland non accade che gli adulti diventano bambini. L'età si perde tra milioni di mattoncini colorati e si scopre che giocare col Lego è un'attività che piace anche a chi ha i capelli bianchi. (CORIS MON2008_10)

(20) Da bambina odiava le bambole e adorava le equazioni. Giocava con i Lego, preferiva la compagnia dei maschi, e risolveva senza difficoltà tutti i problemi aritmetici. (CoLFIS, periodico)

(21) Secondo me una portaerei è solo un giocattolo per un bambino diventato ammiraglio: la barchetta fatta con il Lego è nel frattempo cresciuta anche lei. (CORIS MON2001_04)

(22) Con i Lego le avventure dell'immaginario prendono corpo, danno gusto al gioco del bambino: anche un pezzetto di plastica è un modo per interpretare la realtà. $(\mathrm{LaR})$

(23) Il calcio è un gioco maschio. Campana? Le punizioni? Non capisco: se è così la domenica stiamocene in casa a giocare con la Barbie. (LaR)

(24) Francesca Archibugi: d'accordo giocare con le Barbie. Forse ha ragione lei: è in questo che consiste essenzialmente lo scrivere e il fare del cinema. (LaR)

In generale il numero si presta meno a funzionalità contrastive e oppositive rispetto al genere. Come la categoria del genere, invece, può presentare fenomeni di alternanza non significativa, come hanno illustrato gli ultimi brani.

\subsection{Modificazioni predicative}

Dopo aver analizzato aspetti correlati al nucleo del nesso nominale determinazione $(\S 1.1)$, genere $(\S 1.2 .1)$ e numero $(\S 1.2 .2)$-, in questo paragrafo saranno passati in rassegna alcuni tipi di modificatori che accompagnano i nomi commerciali utilizzati in funzione argomentale, costituendone un arricchimento predicativo (cf. La Fauci 2009: 153).

Le modificazioni che si combinano ai marchionimi comprendono attributi $(\S 1.3 .1)$ e apposizioni $(\S 1.3 .2)$ : i primi tradizionalmente 
di forma aggettivale (ma che si realizzano anche con forme diverse), i secondi di forma nominale (cf. La Fauci 2009: 153-154 e 157).

\subsubsection{Attributi}

(1) Sulla rotta della delusione da Trapani a Tunisi siamo in duecento: un gruppetto sparuto di turisti dalle bionde chiome, un manipolo di giovani tunisini in tuta fiammante e Adidas corazzate che torna in patria per le vacanze [...]. (LaR)

(2) Dietro i Ray-ban marroni gli occhi sono arrossati come la piccola cicatrice che ha sulla fronte. Nicu è il ritratto della madre Elena, un volto affilato che scruta ora con curiosità e un filo di paura i giudici, la gente. (LaR)

(3) Ma mancava il passaggio intermedio: non esisteva ancora una Barbie incinta, pardon, una signora Cuore col pancione e con le doglie. (LaR)

(4) L'estate scorsa l'eroe di "Mani pulite" ha fatto il suo ingresso in paese a bordo di un'Alfa blindata, preceduta da una Croma e seguita da altre due auto, e, come se non bastasse, c'era anche l'elicottero che volteggiava sul corteo. (CoLFIS, periodico)

(5) Francesco Terrone, intanto, sta preparando la sua valigia: stasera carica la Uno bianca e poi via, verso Salerno. (LaR)

(6) Tutto nasce dal progetto annunciato dal manager italo-canadese di separare in due la Fiat attuale. Oggi l'azienda fa capo a un'unica società, la Fiat Spa, che raggruppa il controllo di tutte le attività industriali [...]. (CORIS MON2008_10)

In questi brani i nomi commerciali sono seguiti da un aggettivo qualificativo.

Dal punto di vista combinatorio l'esempio (6) - "separare in due la Fiat attuale" - si distingue dai precedenti in quanto l'articolo, reso necessario dalla presenza del modificatore, può essere soltanto determinativo, diversamente dal precedente la Uno bianca. La struttura ingenera quello che è stato definito "uso modificato" del nome proprio (sul quale cf. la trattazione di Gary-Prieur 2005 e il §1.1.1, supra).

Per gli aggettivi preposti si considerino gli esempi seguenti:

(7) I medici lo hanno ammesso: il cuore di Bartali batte sano e lento come il motore di una vecchia Guzzi. (LaR) 
(8) Entro pochi giorni, fra tutti coloro che avranno inviato i tagliandi, sarà sorteggiata una scattante Fiat Brava versione 1.4 super. (CORIS STAMPAQuotidiani)

(9) La Jeanseria del corso è l'unica che sconta i mitici Levis, un articolo stravenduto che nessuno si sente di ribassare [...]. (CORIS STAMPAQuotidiani)

In questi casi la modificazione attributiva presenta valore non-restrittivo, che in italiano può manifestarsi appunto nell'ordinamento "attributo + nome" contrapposto a "nome + attributo". Si osservino gli esempi (10-12), da contrapporre a (13-15):

(10) La giapponese Sony ha annunciato un progetto per vendere musica su Internet a partire dalla fine di quest'anno. (CORIS STAMPAQuotidiani)

(11) Poco dopo la scorsa Epifania era stata la danese Lego, da trent'anni dominatrice delle costruzioni con mattoncini di plastica, ad annunciare un ridimensionamento del dieci per cento del suo personale in vari paesi. (CORIS STAMPA Quotidiani)

(12) Il governatore Antonio Fazio si rilassa e riduce il tasso di sconto, la prestigiosissima rivista Fortune, Bibbia dei super-ricchi della Terra, addita l'italiana Luxottica di Leonardo Del Vecchio tra i sette titoli al mondo di società non americane da comperare per le eccellenti prospettive 1998. (CORIS STAMPAQuotidiani)

(13) Oldoni e Ronaldo hanno la stessa misura, il 42. E proprio grazie a tale coincidenza, la Nike italiana (sede a Reggio Emilia) che ha un abbinamento col Brescello, ha avuto l'idea di affidare in prova ad Oldoni le scarpe da gioco destinate alle preziose estremità inferiori del fuoriclasse brasiliano. (CORIS STAMPAQuotidiani)

(14) La Olivetti danese sarebbe interessata all'acquisto della Gn Netcom, una società specializzata in comunicazioni elettroniche e network. (LaR)

(15) Ieri, a nemmeno 24 ore dall'annullamento della tournée americana, la Sony inglese ha diffuso un comunicato in cui si legge: "Gli Oasis sono tornati in Inghilterra $[\ldots] ” .(\mathrm{LaR})$

Negli esempi (10-12) - La giapponese Sony, la danese Lego, l'italiana Luxottica - l'aggettivo che precede il marchionimo ha valore non-restrittivo, mentre in (13-15) esso segue il nome e ha valore restrittivo (la Nike italiana, La Olivetti danese, la Sony inglese). 
La posizione postnominale non è peraltro indizio sufficiente per stabilire il valore restrittivo di un attributo, come nel costrutto che segue (Samsung, infatti, è coreana):

(16) L'indice Nikkei perde il $10 \%$, è affondato dal crollo dei mercati di sbocco del made in Japan, dal micidiale rialzo dello yen, dagli avvisi di tagli agli utili della Sony e altri colossi asiatici come la Samsung coreana. (CORIS MON2008_10)

Ha valore restrittivo rispetto al nome commerciale, invece, il toponimo Usa in (17): ${ }^{39}$

(17) L'impianto verrà costruito, dal prossimo autunno, non molto distante da Chicago, dove ha sede la Barilla Usa. (CORIS STAMPAQuotidiani)

Non solo aggettivi, infatti, si combinano ai nomi commerciali in qualità di modificazioni attributive. Si ritrovano anche espressioni di vario tipo, formalmente diverse ma funzionalmente equivalenti agli aggettivi qualificativi (cf. La Fauci 2009: 153):

(18) "In effetti c'è stato un piccolo imprenditore edile che mi ha costretto ad ordinare in azienda una Jacuzzi color Ferrari Testarossa [...]”. (LaR)

(19) E invece è bella, una domenica così: di sport vero, tutti sul prato come su un lettone d'erba, con i cani che corrono, la bora che frusta i cespugli, e quella ragazzina felice di settant'anni, sì quella con le Superga con i buchi e gli occhi di Alida Valli, che studia le galassie e sogna di essere Zorzi. (LaR)

(20) Ieri mi sono visto capitare in casa un giornalista della tv svizzera che mi ha chiesto se non mi vergognavo a mangiare la gomma e a buttare la carta per terra, su un prato così bello. La prima reazione è stata quella di ruttargli nel microfono, ma lui ha capito e mi ha subito teso un Toblerone da un chilo: mi sono intenerito. (LaR)

(21) “È venuto uno della Rai” dice Ludovico del Parini, "voleva sapere il significato politico dei vestiti e delle fogge, le creste biondo-rosa delle ragazze punk, i giubbotti di cuoio e gli stivaletti borchiati dei testa rapati, le Timberland dei paninari. $[\ldots] ” .(\mathrm{LaR})$

39 Diversamente dagli aggettivi visti sopra, Usa, in funzione attributiva, non può che seguire il nome. 
Anche i nomi che specificano il modello di un prodotto - nomi commerciali in senso lato, senza considerare l'eventuale statuto di marchio registrato - sono annoverabili tra gli attributi che modificano il marchionimo:

(22) Le scarpe da ginnastica sono ok, ma solo se della marca giusta, al momento: Puma States, Adidas Gazelles e Converse One Stars. Ma l'anno prossimo saranno diverse. (CORIS MISCVolumi)

(23) E sempre ieri pomeriggio, negli uffici del Nucleo Operativo di via Valfrè, è stato sentito anche l'amico che prestava abitualmente il Suzuki Vitara nero a Gianni. (CoLFIS, quotidiano)

(24) Questione di pochi istanti e mentre già il blindato filava spedito in direzione di Chivasso (dove si sarebbe fermato solo davanti alla caserma dei carabinieri) sul luogo del fallito assalto sopraggiungeva una Lancia Dedra, rubata anche questa. (CoLFIS, quotidiano)

\section{Tali attributi possono nominalizzarsi:}

(25) Le All Star celesti, con la piccola stella rossa che centra il rotondo di gomma sulla caviglia, scendono dalla Vespa e toccano agilmente terra. (Moccia 2004: 20)

(26) A conferma del successo registrato da questo modello, va detto che la Vitara occupa attualmente la seconda posizione nella classifica delle fuoristrada più vendute, dopo la Mitsubishi Pajero. $(\mathrm{LaR})^{40}$

(27) Senza dubbio più preziosa della vecchia Prisma e venduta a un prezzo che solo qualche anno fa sarebbe stato assai vicino a quello di una Thema, la Dedra ha subito raccolto un buon successo sul mercato nazionale [...]. (LaR)

Hanno funzione attributiva anche i complementi interni al nesso nominale, introdotti perlopiù dalla preposizione di (cf. La Fauci 2009: 163), già visti nel $§ 1.1 .1$ :

(28) La Vespa della Piaggio, progettata nel 1945 da Corradino D'Ascanio, e la Lambretta disegnata nel 1947 da Cesare Pallavicino per l'Innocenti, non sono stati esclusivamente dei grandi successi commerciali [...]. (CORIS STAMPASupplementi)

40 Si noti la variazione di genere: nel corpus LaR, il marchionimo (Suzuki) Vitara è sia maschile che femminile. 
(29) Nel 1998 è stato annunciato un rinnovamento: la Barbie del nuovo millennio starà in piedi, il seno e fianchi saranno leggermente ridotti e la vita appena allargata, ma comunque sarà sempre ben diversa da Action Woman. (LaR)

(30) Ad agosto 2004, Mercolli acquista un cellulare Ericsson T 230 alla Migros di Sant'Antonino. (SM 6/05)

Tali attributi sono modificazioni interpretabili di volta in volta come rapporti tra prodotto e produttore (La Vespa della Piaggio: predicazione non-restrittiva), determinazioni temporali (la Barbie del nuovo millennio, con attributo a valore restrittivo), determinazioni spaziali (alla Migros di Sant'Antonino, essa pure di tipo restrittivo).

\subsubsection{Apposizioni}

(1) Anche a livello mondiale il gruppo Ferrero si difende bene perché è preceduto solo dai colossi Nestlé e Mars, con i quali sta combattendo una dura battaglia a colpi di spot televisivi. (CoLFIS, periodico)

(2) Il colosso americano Kodak porta oggi sul mercato una macchina fotografica digitale da 1,2 milioni di pixel. (CORIS PRACCRiviste)

(3) Berlusconi con la sua Fininvest, e con i potenti alleati Fiat e ENI, è stato sconfitto (con una offerta da 750 miliardi presentata dal consorzio Omnitel-Pronto Italia) nella gara per scegliere il gestore privato dei telefonini che si affianca alla SIP. (CoLFIS, quotidiano)

(4) Una grande festa, ma anche un business a parecchi zeri: la moda ha ormai omologato Halloween a una sorta di festa del papà o di San Valentino [...]. Secondo alcuni è una resa incondizionata all'imperialismo culturale americano, se è vero che addirittura la rossa catena Coop ha allestito il reparto "Felice Halloween" con zucche da illuminare e festoni-pipistrello. (CORIS STAMPAQuotidiani)

In questi brani, nomi comuni e nessi nominali precedono il marchionimo e lo modificano predicativamente. Si tratta di apposizioni interne (cf. La Fauci 2009: 157-158). Sovente ricorrono con tale funzione nomi comuni quali azienda, ditta, produttore: 
(5) Così i 70 artisti di tutto il mondo invitati dalla Biennale non usa ad ospitarli, sono spesati di vitto e alloggio dalla Fondazione Prada, mentre l'azienda Prada sponsorizza quattro mostre parallele organizzate dal Comune. (LaR)

(6) La ditta Codemi, che già s'era aggiudicata gli appalti per il carcere di Voghera, vince anche questa gara. (LaR)

(7) I produttori L'Oréal e Schwarzkopf, messi di fronte ai risultati del test, affermano di rispettare la legge. (SM 5/2010)

La concezione tradizionale identifica in questi costrutti il nome proprio come nucleo, del quale il nome comune sarebbe una modificazione. Secondo altri studiosi, invece, la natura predicativa del nome proprio e quindi il particolare rapporto tra i due nomi - definibile alla stregua di una predicazione denominativa: "la ditta chiamata Codemi" - invitano a definire il nome comune non come apposizione interna, bensì come nucleo del nesso, rispetto al quale il nome proprio (di marca) costituisce "una modificazione attributiva, di tipo particolare, in quanto denominativa, ma sostanzialmente non dissimile al caso che presenterebbe un banale aggettivo" (La Fauci 2009: 158). Per Noailly (1990: 143), costrutti quali le colonel Kadhafi (con patronimico), la firme Okamoto (con nome d'impresa) o la marque Daddies (con nome di marchio) presentano delle "relations d'identification"; in essi il nome proprio funge da attributo (épithète) del nome comune (cf. inoltre Jonasson 1994: 91-92). Questi costrutti saranno trattati più ampiamente nel §2.1.

Ricorrono in grande numero apposizioni esterne, spesso complesse e articolate:

(8) Se la fortuna ha sollevato di poco la benda speriamo abbia colpito qualche operaio della Goodyear, la fabbrica di pneumatici che dista pochi chilometri e che dal prossimo febbraio chiuderà i battenti lasciando a casa circa mille persone. (LaR)

(9) [...] così è stato aggredito ieri pomeriggio, mentre lavorava in azienda, Mario Ponta, 35 anni, operaio della Facco, una grossa società padovana di Campo San Martino operante nel settore delle attrezzature avicole. (LaR)

(10) Bruno era un padroncino: aveva acquistato insieme ai fratelli tre furgoni, con i quali girava il Veneto per conto della Aia, un'azienda di allevamento di pollame. (LaR) 
(11) L'anno nuovo è iniziato con un grosso punto interrogativo per la Guinness, la società britannica produttrice tra l'altro dell'omonima birra scura: chi ha acquistato un rilevante pacchetto di oltre 2 milioni di sue azioni (per un valore di 7,5 milioni di sterline) lo scorso aprile, quando la Guinness rilevò la Distillers, dopo un'accanita battaglia con la Argyll? (LaR)

(12) Piace sempre di più all'estero l'espresso "made in Italy", che trova grandi estimatori anche in Giappone. È uno dei dati che emergono dal bilancio della Lavazza, leader italiana nel settore del caffe [...]. (LaR)

(13) Michael [Jordan], infatti, ha da tempo una esclusiva miliardaria con la Nike, la grande rivale della Reebok nell'abbigliamento sportivo [...]. (CoLFIS, quotidiano)

(14) Parte integrante nell'iniziativa è lo sponsor del cantante, la Pepsi Cola, che ha permesso di fissare il prezzo del biglietto del "Dangerous Tour" a 40 mila lire [...]. (CoLFIS, quotidiano)

I riferimenti di apposizione esterna e nome cui essa è attribuita coincidono; per questo motivo all'apposizione esterna si riconosce una natura di modificazione predicativa (La Fauci 2009: 158-159). Nella sua complessità essa veicola informazioni (nuove) relative al nome commerciale (cf. Vande Casteele 2012: 94; Noailly 1990: 143).

Anche i marchionimi utilizzati in riferimento a prodotti - i casi individuati nel $\S 1.1 .3$ - possono ricorrere accompagnati da una modificazione esterna che specifica il tipo di prodotto (nome comune "generico"):

(15) Negli ultimi anni, sono già riusciti a creare negli Stati Uniti un clima ostile al fumo. Non solo è vietato accendere la Marlboro (la sigaretta più venduta) o un'altra marca, negli uffici pubblici o nei taxi, ma anche in molti salotti privati vige la stessa regola. (LaR)

(16) Era l'elemento-chiave per riconoscere un turista tedesco, cioè una persona che non bada all'eleganza. Oggi è diventata una calzatura cult [...]. Parliamo de $i$ Birkenstock, i sandali anatomici con la suola di sughero, ormai prodotti in decine di versioni modaiole, la più gettonata delle quali è, per l'estate 2004, quella color argento. (LaR online ${ }^{41}$ )

$41<$ ricerca.repubblica.it/repubblica/archivio/repubblica/2004/07/10/sandalida-turista-tedesco-le-signore-vanno.html?ref=search $>$ (18.2.2015). 
Se si comprendono anche questi tra i casi di apposizioni esterne, si potrebbe, per converso, trattare le strutture $i$ sandali Birkenstock o la sigaretta Marlboro come apposizioni interne. Ciò, peraltro, si giustificherebbe con il fatto che in determinati casi vi è effettivamente tanto coincidenza di riferimento quanto la possibilità, per i due elementi del nesso nominale, di ricorrere autonomamente: la Marlboro/la sigaretta (Marlboro). In merito alla questione della predicazione denominativa ci si può allora chiedere in che misura le Adidas siano 'scarpe chiamate Adidas', come la Adidas è 'la ditta che ha nome Adidas' e il presidente Mitterrand è 'il presidente chiamato Mitterrand'. Nel caso del valore di nome di prodotto potrebbe trattarsi pure di una relazione diversa: 'scarpe prodotte da Adidas'o altro. Ma questo ha un'importanza secondaria; centrale è invece l'osservazione per cui scarpe (come ditta, come presidente) ha funzione di argomento, rispetto al quale il nome commerciale Adidas funge da predicato. Questo vale, lo si ricordava sopra (§1.1.4), anche nei casi in cui il marchionimo compare in funzione argomentale (le Adidas): si tratta infatti, nell'ipotesi qui sostenuta, di un predicato che si è nominalizzato.

\section{Conclusioni}

L'analisi condotta in questo capitolo ha permesso di mettere in evidenza alcune caratteristiche combinatorie dei nomi commerciali che ricorrono in funzione di argomento, arricchendo notevolmente le descrizioni dei marchionimi rispetto a quanto è presente nella letteratura sul tema. Si conferma l'importanza di un'analisi basata su dati reali, che permette di smentire assunti ricorrenti, in particolare quelli riguardanti presenza o assenza "obbligatorie" di determinatori.

Nella prospettiva di un'analisi funzionale, sistemica, si è tenuto conto non solo dei rapporti in praesentia ma pure di quelli paradigmatici, dal momento che gli elementi della lingua, secondo l'insegnamento di Saussure, valgono anche per quello che non sono (e che potrebbero essere). Assenza e presenza di determinatori non sono considerati in maniera assoluta, bensì nel quadro delle relazioni paradigmatiche $\mathrm{e}$ 
sintagmatiche che si manifestano nella proposizione. L'opposizione tra i costrutti "Helmut Newton calza Reebok che sembrano nuove" e "Altobelli calza Adidas", per es., permette di osservare che nel primo caso il marchionimo ha, univocamente, funzione di oggetto diretto, quindi argomentale, mentre il secondo si può leggere come un uso avverbiale più che nominale. Si può stabilire un'equivalenza tra il primo costrutto e "calza delle Reebok": la determinazione zero è funzionalmente equivalente a un articolo indeterminativo plurale.

Un'ampia parte dell'analisi è stata dedicata ai nessi Det Nmc per i quali si riconosce una correlazione con un luogo comune. Oltre alla questione dell'articolo si è discussa in particolare l'ipotesi dell'ellissi, chiamata in causa per spiegare il genere correlato al nome commerciale; se, intuitivamente, essa pare reggere, dal profilo argomentativo si tratta di un'opzione debole, essendo perlopiù non verificabile. Non si può infatti presupporre la conoscibilità (e reperibilità) di un eventuale nome generico cancellato. Più plausibile, come si è argomentato, è l'ipotesi di un processo di nominalizzazione alla base dei costrutti Det $N m c$ : esso vede i nomi di marca come predicati che si sono argomentalizzati. Genere (soprattutto) e numero, dal canto loro, vanno valutati come tratti funzionali alla differenziazione di valori (il Peugeot/la Peugeot), quando non siano semplicemente varianti libere (i Birkenstock/le Birkenstock). Ancora, si propone di sostituire il concetto di ellissi con quello di pertinenza al fine di spiegare i costrutti Det Nmc: in un brano relativo all'acqua minerale le uniche variabili sono i nomi commerciali delle acque descritte, predicato di un argomento - acqua minerale, appunto - che non è necessario ripetere.

Un altro aspetto fondamentale riguarda la differenza tra una visione ontologica, frequente negli studi sui marchionimi, e quella sintattico-combinatoria qui proposta. Come l'analisi dovrebbe aver mostrato con chiarezza, non sono i nomi commerciali a manifestare determinate proprietà morfosintattiche o semantiche, bensì i costrutti nei quali ricorrono. Una rete di relazioni sintagmatiche e paradigmatiche determina i vari nessi nella loro specificità e in opposizione ad altri. Non sono dunque i singoli nomi che, in quanto appartengono a varie categorie referenziali (prodotto, azienda ecc.), hanno diverse proprietà combinatorie nella lingua; è invece la sintassi a farne scaturire valori 
diversi. I nessi nominali descritti in questo capitolo non sono enti ma processi; lo si è messo in luce in particolare in relazione alla questione dei costrutti le Nike, $i$ Wrangler, ma quanto affermato è valido in generale per i nomi commerciali (in questo accomunabili ai nomi propri prototipici).

Nei costrutti analizzati, insomma, si riconosce ai nomi commerciali una natura predicativa rispetto al determinatore, che ne costituisce la specificazione argomentale. Tale carattere di predicato emerge in maniera manifesta nel capitolo che ora ci apprestiamo ad affrontare, dedicato appunto agli usi predicativi: in un costrutto come scarpe Adidas il nome commerciale è il predicato - stavolta non nominalizzato del nome comune, che funge da argomento. 



\title{
2. Nessi nominali: usi predicativi
}

\author{
Introduzione
}

(1) Quale efficacia può avere un testimonial come Alberto Tomba che si affaccia in tv quasi contemporaneamente per pubblicizzare il corso della De Agostini, le tagliatelle Barilla, il marchio Marlboro, i concessionari Fiat e la Fiat Tipo? (LaR)

La variegata attività promozionale dello sciatore bolognese è descritta in questo brano attraverso cinque nessi nominali, diversi per struttura formale. Ricorrono il corso della De Agostini, con nome commerciale preceduto da preposizione (v. cap. 3), e la Fiat Tipo, esempio di marchionimo (complesso) in funzione argomentale, come quelli studiati nel cap. 1. Le strutture restanti - le tagliatelle Barilla, il marchio Marlboro, $i$ concessionari Fiat - rappresentano invece tre usi del nome di marca che in questa sede sono definiti predicativi (v. Parte II, §1.2) e che costituiscono l'oggetto del presente capitolo: il costrutto $N \mathrm{Nmc}$, in cui il marchionimo funge da modificatore rispetto a un nome comune o nesso nominale.

Si tratta di un costrutto che ricorre in proporzioni fortemente variabili rispetto agli usi argomentali dei nomi commerciali. In genere gli usi argomentali sono di gran lunga preponderanti: nel romanzo di Moccia che sarà analizzato nel cap. $7 \mathrm{i}$ costrutti con marchionimo in funzione predicativa sono solo una decina su quasi quattrocento; uno spoglio dei costrutti con Lavazza all'interno del corpus LaR restituisce invece un quinto di strutture $N N m c$. Ma si dà anche il caso opposto: in un articolo di quotidiano reperito nei dati $C o L F I S$, su sedici ricorrenze di Fiat, dodici modificano un nome comune o nesso nominale (aziende Fiat, consiglio d'amministrazione Fiat ecc.).

La combinazione di nome comune e nome proprio prototipico è stata studiata da linguisti soprattutto di area francofona (Kleiber 
1985; Noailly 1990; Jonasson 1994 e 1995). ${ }^{1}$ Per i marchionimi, singoli accenni si ritrovano in Migliorini (1927), nelle analisi di Folena (1964) e Calabrese (1974) sulla lingua della pubblicità e in Grass (2002), che ne tratta in prospettiva interlinguistica.

Per entrare nel vivo della questione si consideri il brano seguente, inventato ma plausibile:

(2) Il bambino Roberto Cavalli è atteso alla cassa del supermercato.

Soggetto della proposizione è il nesso nominale Il bambino Roberto Cavalli: un costrutto in cui si riconosce un rapporto d" "identificazione" tra nome comune e nome proprio, secondo la classificazione di Noailly (1990: 132 e seguenti) ripresa da Jonasson (1994). Costrutti simili sono per es. il presidente Obama, il cardinal Martini.

Le due studiose non sono concordi nel definire il ruolo del nome proprio in questo tipo di struttura: Noailly (1990) gli riconosce funzione di attributo (in francese épithète), mentre per Jonasson (1994: 93) esso è una delle due teste di un sintagma nominale complesso. Entrambe concordano, peraltro, nell'osservare che i due nomi sono co-referenziali; ne è una prova il fatto che vale "Roberto Cavalli è un bambino" (cf. Noailly 1990: 135). Il nome comune, inoltre, è il nucleo del nesso nominale: il bambino Roberto Cavalli è un bambino (cf. Kleiber 1985: 4). ${ }^{2}$ Si può aggiungere poi - ed è, questo, un punto cruciale per l'approfondimento dei nomi propri - che il bambino in questione "è chiamato Roberto Cavalli". Il rapporto tra nome comune e nome proprio è dunque di tipo denominativo (cf. Noailly 1990: 136).

Si confronti ora il brano (2) con il seguente:

(3) Il bambino Roberto Cavalli è stilish [sic] $\left(<\right.$ www.sfilate.it $>^{3}$ [titolo])

È il titolo di un articolo dedicato alle collezioni kidswear primavera/ estate 2012 di Roberto Cavalli. Vi ricorre, in apparenza, lo stesso nesso

1 Gli studi citati si muovono anche negli ambiti semantico e pragmatico ma in questa sede ci si limiterà perlopiù a osservazioni sintattiche.

2 Si veda inoltre Harris (1952: 21), che stabilisce un'equivalenza tra $N_{1} N_{2}$, "with a primary stress on each $N$ ", e $N_{1}=N_{2}$ : "The pressure $P$ increases is equivalent to The pressure increases and $P$ increases".

$3<$ www.sfilate.it/132542/il-bambino-roberto-cavalli-piu-stilish> (3.11.2014). 
nominale dell'esempio (2), sempre in funzione di soggetto: Il bambino Roberto Cavalli. I due costrutti presentano però importanti differenze sintattiche, oltre a quelle referenziali evidenti a una prima osservazione.

Innanzitutto i due nomi, comune e proprio, non sono equivalenti quanto alla possibilità di ricorrere isolatamente come soggetto della proposizione. Il bambino è stylish potrebbe forse funzionare, ma Roberto Cavalli è stylish avrebbe un significato diverso. Non c'è inoltre coincidenza con il costrutto "Roberto Cavalli è un bambino". Infine la relazione tra i due componenti del nesso non è di tipo denominativo: non è questione di un bambino chiamato Roberto Cavalli (ma, eventualmente, di un bambino "firmato Roberto Cavalli"; cf. §4.5).

In sostanza, il nome proprio si presenta in (3) come un attributo, un modificatore; non un nome utilizzato argomentalmente, nucleo del nesso nominale, ma un elemento retto e legittimato dal nome comune che lo precede. Pur trattandosi di un nome dal punto di vista formale, gli si riconosce la funzione sintattica usualmente assunta dall'aggettivo (cf. Jonasson 1994: 92): nell'esempio (3) Roberto Cavalli è un predicato, non un argomento.

Come si è constatato con questi esempi e si approfondirà in questo capitolo, le strutture $N N m c$, formalmente simili, possono celare tipi sintattici diversi e, di conseguenza, interpretazioni diverse (cf. l'analisi di Kleiber 1985). Così, per riprendere l'esempio citato in apertura, si può affermare che le tagliatelle Barilla e $i$ concessionari Fiat corrispondono funzionalmente all'esempio (3), mentre il marchio Marlboro somiglia piuttosto al tipo esemplificato in (2), in cui il nome proprio sta in rapporto di denominazione con il nome comune: Marlboro è un marchio ed è il nome del marchio, come Roberto Cavalli è un bambino e al contempo il nome del bambino.

Mentre per l'analisi degli usi argomentali dei marchionimi è centrale l'aspetto della determinazione, con gli usi predicativi la descrizione di proprietà combinatorie si concentra sostanzialmente su tre punti:

1) equivalenza tra $N N m c$ e $N m c$ è $N$ ("il marchio Marlboro" = "Marlboro è un marchio"); 
2) equivalenza tra $N N m c$, con marchionimo in funzione attributiva, e $N$ di(Det) Nmc, con complemento di specificazione ("le tagliatelle Barilla" = "le tagliatelle della Barilla");

3) equivalenza tra costrutti con marchionimo usato predicativamente $(N N m c)$ e usi argomentali dei marchionimi (Det Nmc), per es. "le sigarette Marlboro"/"le Marlboro".

La prima proprietà elencata permette di distinguere tra due usi predicativi dei nomi commerciali: i predicati di denominazione (o, con Jonasson 1994, di "identificazione") sono i soli che la presentano. Nel §2.1 sono trattate le strutture più ricorrenti per questo tipo, contenenti i nomi comuni ditta, azienda, marchio e simili, che fungono da classificatori. Nel caso di azienda è pure plausibile un uso argomentale dell'attributo, con determinatore a variante libera: l'azienda Barilla/la Barilla.

L'equivalenza tra uso attributivo e complemento preposizionale contraddistingue i costrutti con relazione predicativa non denominativa (§2.2), in cui i nomi commerciali, secondo la terminologia di Jonasson (1994: 105), fungono da attributi di determinazione. In questa struttura il nome comune designa il "referente primario" (Jonasson 1994: 106) mentre il nome proprio, retto dal nome comune, svolge funzione di attributo rispetto a esso e lo determina. Si distinguono tre gruppi principali in base al tipo di nome comune contenuto nel costrutto: nomi concreti, nomi astratti, nomi [+ umani]. Per tutti e tre è possibile una parafrasi $\mathrm{Nc}$ di Nmc.

Il §2.2.1 studia i casi come le tagliatelle Barilla e le scarpe Adidas, in cui la relazione si configura nei termini di "prodotto-produttore". Caratteristica di tali costrutti - perlomeno di una parte di essi è la possibilità di un uso argomentale del marchionimo preceduto dal determinatore (le Adidas). Sono poi considerati i costrutti che, al contrario, non hanno un equivalente di uso argomentale: il nome commerciale non può costituire il nucleo del nesso, non è nominalizzabile. Il costrutto con nome umano e marchionimo (il pilota Mercedes), per es., si differenzia radicalmente dalla struttura con antroponimo (il pilota Schumacher), nella quale il nome comune è apposizione del nome proprio ed entrambi possono ricorrere singolarmente con lo stesso significato del nesso nominale complesso; "Mercedes è un pilota" risulta indubbiamente più marcato di "Schumacher è un pilota". Così anche 
nel caso dei nomi non-umani, sia concreti che astratti: lo spot Parmacotto, la vicenda Fiat. Per i nomi astratti si può osservare, con Jonasson (1994: 107-108), che l'interpretazione del nesso è fortemente soggetta a conoscenze di tipo pragmatico o enciclopedico.

Le analisi degli studiosi citati si rivolgono perlopiù a costrutti $N$ $\mathrm{Npr}$ che contengono un nome personale. Con le indagini presentate in questo capitolo si intende fornire un complemento a tali ricerche riguardante un altro settore dei nomi propri, non del tutto sovrapponibile a quello degli antroponimi. Nondimeno, sarà possibile individuare anche in questo campo delle regolarità strutturali, oltre ad arricchire indirettamente la descrizione fornita per i nomi propri prototipici.

\subsection{Marchionimo come predicato di denominazione}

\subsubsection{Il costrutto azienda Nmc}

Nella maggior parte dei casi, i costrutti l'azienda Nmc, la ditta Nmc e simili si configurano nei termini di una relazione di tipo denominativo, per cui nome comune e nome commerciale sono entrambi nuclei del nesso e il marchionimo svolge funzione di predicato di denominazione. Come si vedrà, però, ciò non vale in assoluto per tutti i nessi azienda Nmc. Lo studio delle proprietà combinatorie dei costrutti permette di distinguere tra le modificazioni denominative - le più frequenti - e quelle attributive.

(1) Tensione anche sul versante sindacale: in un comunicato stampa diffuso ieri, l'esecutivo del comitato di redazione rileva che l'azienda Mondadori ha dimostrato totale chiusura sulla Carta dei diritti dei giornalisti e sottolinea la sorprendente contraddizione tra questo atteggiamento e gli apprezzamenti manifestati da Berlusconi il 12 febbraio scorso. (LaR)

(2) [Giovanni Enriques] Preferì dopo la laurea in ingegneria nel 1930, abbandonare l'amicizia con Fermi per entrare nell'azienda Olivetti, dove dall'apprendistato dei primi anni passò in seguito alla carica di direttore generale. (LaR) 
(3) Ho letto con non poca inquietudine la notizia della discriminazione perpetrata a Foggia, dall'azienda Barilla, nei confronti delle persone che portano tatuaggi, per un'eventuale loro assunzione, giustificando il tutto con non meglio specificate motivazioni sanitarie. (LaR)

(4) Il primo operaio della ditta Montano è entrato nel pozzetto, aggredendo i tubi interrati, quando, forse manovrando una valvola, si è sprigionata una fuga di gas, che si deve essere formata in testa alle condutture. (LaR)

(5) La ditta Codemi, che già s'era aggiudicata gli appalti per il carcere di Voghera, vince anche questa gara. (LaR)

I nessi azienda/ditta Nmc formano un'importante sezione entro gli usi di marchionimi come predicati di denominazione. Come nomi comuni vi ricorrono, oltre ad azienda e ditta, anche impresa, società e altri simili, utilizzati spesso come sinonimi; tutti, si noti, di genere femminile. ${ }^{4}$

In alcuni casi il nome commerciale modifica non soltanto un nome ma un nesso nominale composto di nome comune e aggettivo o altro modificatore:

(6) Nello stesso periodo anche l'impresa informatica Fujitsu aumenterà la produzione di questo tipo di microchip accrescendola del 20 per cento e portandola a un totale mensile di due milioni di unità. Presto, secondo la Kyodo, anche le aziende elettroniche Sony e Aiwa rivedranno i piani di produzione degli stabilimenti britannici che fabbricano apparecchi e schermi televisivi e impianti stereofonici hi-fi. La società produttrice di batterie Yuasa ha già deciso invece di portare la produzione da quattro a sette milioni di unità. (CoLFIS, quotidiano)

Il nesso modificato dal marchionimo si comporta sintatticamente come un'apposizione interna di quest'ultimo (v. §1.3.2). Ciò va di pari passo con quanto osservato nell'introduzione al presente capitolo sulla scorta della letteratura sul tema: in questi costrutti, nome comune (con eventuali modificatori) e nome proprio (di marca) sono interpretabili entrambi come nuclei del nesso nominale. Alla struttura "La società produttrice di batterie Yuasa" corrisponde "Yuasa è una società produttrice di batterie"; si tratta dell'equivalenza tra $N N m c$ e $N m c$ è $N$, segnalata come indicatore del carattere di predicazione nominale del marchionimo rispetto a società o azienda. In termini sintattici si può

4 Come azienda funzionano sintatticamente anche casa editrice, gruppo editoriale e simili, qui non approfonditi. 
fare un paragone con la struttura in cui un antroponimo segue un nome comune indicante un titolo (professionale): a l'ingegner Gadda corrisponde Gadda è un ingegnere.

Vi è somiglianza con i costrutti in cui il marchionimo svolge funzione argomentale ed è modificato da un'apposizione esterna (cf. $\S 1.3 .2)$ :

(7) La Moto Guzzi, azienda produttrice di motociclette, ha nominato il nuovo amministratore delegato, Oscar Cecchinato, che sarà responsabile della gestione complessiva dell'azienda di Mandello Lario. (LaR)

(8) La previsione è della Lavazza, l'azienda di Torino che ha il 44 per cento del mercato italiano del caffè destinato alle famiglie, allarmata dal comportamento dei fondi di investimento. (LaR)

(9) Marone aveva parlato tra l'altro delle tangenti pagate dalla Ferrarelle - la nota azienda di acque minerali - per ottenere il via libera al rientro in commercio della sua Acqua Fabia, che era stata sequestrata cautelarmente dalla magistratura. (LaR)

L'apposizione corrisponde alla predicazione che si riscontra nei costrutti copulari: Ferrarelle è la nota azienda di acque minerali. Al proposito si osserva, nei dati, un rapporto di equivalenza tra l'azienda/ditta Nmc e la Nmc. Le due strutture si alternano, anche nella stessa porzione di discorso (ess. 12-13), manifestando un valore comparabile:

(10) Anche la ditta Wuber di Medolago ha rilasciato ieri un comunicato. (LaR)

(11) Nel 1976 [Giuseppe Beretta] ha fondato la Wuber, primo stabilimento italiano studiato per produrre solo wurstel. (LaR)

(12) Il posto più alto del podio, con il giudizio "buono" è occupato dalla ditta Head per entrambi i suoi modelli di sci, ma si trova a pari merito con la Fischer per quanto riguarda quelli per le donne. (SM 6/05)

(13) La Rolls Royce esiste ancora come azienda, ma non è più quella che produce le auto più [sic] di lusso più famose del mondo [...]. Le auto che arrivano in commercio con il marchio Rolls Royce insomma non sono più prodotte dalla Rolls Royce ma da una divisione della Vickers. L'azienda Rolls Royce invece si è specializzata nei motori per aerei civili e militari e in una serie di produzioni analoghe. (LaR) 
Chiaramente non si tratta di una sinonimia assoluta, per cui la ditta Wuber e la Wuber sarebbero sostituibili l'una con l'altra in ogni contesto; sono le relazioni sintagmatiche e paradigmatiche a costituire il valore del nesso e dei suoi componenti.

Il costrutto l'azienda/la ditta Nmc esprime quanto vale, implicitamente, anche per la Nmc: Rolls Royce e Fischer sono, in questi contesti, nomi di un'azienda. (Per gli aspetti morfosintattici del costrutto la $N m c$ come equivalente di l'azienda $N m c$, in particolare la determinazione come variante libera, si rimanda al §1.1.2.)

Si noti che, quando ricorre con il valore definibile 'azienda', il marchionimo manifesta di norma genere femminile; questo avviene anche quando - come nel brano seguente - esso è seguito da un'apposizione esterna il cui nucleo è un nome maschile:

(14) Sull'auto elettrica studi e realizzazioni sono assai più ampi e vanno dalla Impact della Gm [...] alle soluzioni di Ford, Volkswagen, Chrysler [...], a quella della Electric Power di Tokyo che usa una batteria al sodio-zolfo prodotta da AseaBrown Boveri, e addirittura a quella della Swatch, il ben noto produttore di orologi. (LaR)

Vi sono autori (per es. Grass 2002: 221) che motivano il ricorrere dell'articolo femminile singolare come una ripresa del genere e numero di ditta o azienda. Da un punto di vista combinatorio, il tipo la Nmc può però essere inteso anche come una nominalizzazione dell'attributo, similmente a quanto discusso nel §1.1.4.

Si torni ora al costrutto "l'azienda Nmc". Un'analisi delle sue ricorrenze sintattiche e delle sue peculiarità combinatorie permette di evidenziare che ciò che fenomenicamente appare come lo stesso costrutto può essere manifestazione di due tipi diversi di relazioni tra nome comune e marchionimo.

(15) Due i nomi finiti a verbale: uno è quello della Breda costruzioni ferroviarie, colosso del gruppo Efim, che forniva i treni alla metropolitana. Il secondo è quello dell'Iveco, l'azienda Fiat il cui marchio luccica sugli autobus pubblici milanesi. (LaR)

(16) Il varo dell'integrazione chimica, comunque, deve essere accompagnato secondo il sindacato da un intervento dei due ministri per verificare la possibilità di recuperare, nell'ambito del nuovo polo, le produzioni di Montefluos, l'azienda Montedison per i derivati del fluoro. (LaR) 
L'azienda Fiat, l'azienda Montedison, l'azienda Eni: da un punto di vista combinatorio, sono costrutti come quelli visti nel §2.1.1? L'analisi permette di rispondere di no. In primo luogo, tali nessi sono in rapporto sintagmatico con un altro nome commerciale, che essi modificano in qualità di apposizione $(15,16)$ o che ricorre in un costrutto identificativo (17). In secondo luogo, non vi è coincidenza con l'uso argomentale: Iveco è un'azienda Fiat ma non è una Fiat, e sarà difficile che ricorra l'espressione la Eni prediletta risulta la Snam, fatto salvo il caso di una ripresa all'interno del testo, per cui l'articolo di la Eni assumerebbe valore pronominale.

Negli esempi (15-17), dunque, il marchionimo ha un rapporto diverso con il nome comune azienda rispetto a quello visto nel paragrafo precedente: lo modifica come un normale attributo, come un aggettivo di relazione, non come modificazione nominale. L'azienda Fiat non vale 'l'azienda chiamata Fiat'. Se l'aggettivo corrispondente esistesse e fosse di uso corrente, si potrebbe affermare che "l'Iveco è l'azienda fiatese il cui marchio luccica sugli autobus": un aggettivo a tutti gli effetti. Ciò non vale per "la ditta Wuber ha rilasciato un comunicato": qui, infatti, il marchionimo funge da predicazione nominale, per cui l'aggettivo wuberiana non sarebbe legittimato a ricorrervi.

Si riscontra inoltre una corrispondenza tra l'azienda $N m c$ degli esempi (15-17) e l'azienda di Nmc, con complemento preposizionale:

(18) I francesi preferiscono le joint venture: la Lyonnaise des Eaux ha firmato un accordo con Fisia, l'azienda della Fiat che si occupa di impiantistica ambientale, dando vita alla Segesta, società che gestisce impianti per scarichi liquidi e rifiuti solidi. (LaR)

È chiaro che non si allude qui all'“azienda Fiat" tout court (ossia "la Fiat"), bensì a una particolare azienda del gruppo Fiat, che è specificata nella relativa seguente e che porta un nome diverso, Fisia. La coincidenza funzionale con la struttura $N d i \mathrm{Nmc}$ mette in luce la natura attributiva del marchionimo nei casi evidenziati di $N \mathrm{Nmc}$, mentre per le predicazioni nominali non vi è coincidenza con la struttura $N$ di Nmc. 
Quanto osservato in merito alla struttura, o meglio, alle differenti strutture "azienda $N m c$ " è un monito a "diffidare del quasi-uguale [...], del praticamente identico, del pressapoco, dell'oppure, di tutti i surrogati e di tutti i rappezzi", come riassume il narratore di Potassio, ne Il sistema periodico di Primo Levi, a seguito di uno sfortunato esperimento chimico: "Le differenze possono essere piccole, ma portare a conseguenze radicalmente diverse, come gli aghi degli scambi; il mestiere del chimico consiste in buona parte nel guardarsi da queste differenze, nel conoscerle da vicino, nel prevederne gli effetti. Non solo il mestiere del chimico" (Levi 2014: 58).

Questo secondo tipo di struttura "azienda $N m c$ ", senza rapporto denominativo tra $\mathrm{i}$ due membri, ricorre naturalmente (perlopiù) con nomi comuni diversi da azienda: lo si vedrà in seguito (cf. §2.2). Per tale costrutto si può ipotizzare una relazione di possesso - in senso lato - tra marchionimo e nome comune: non "Fiat è un'azienda" bensì "Fiat ha, tra le altre, un'azienda chiamata Fisia/Iveco". In questo senso Fisia e Iveco sono delle aziende "fiatesi". Lo si osserva pure nell'esempio seguente:

(19) Nella ripartizione di questi "paracaduti sociali" la Fiat fa la parte del leone: al gruppo torinese, infatti, sono stati accordati 4.000 prepensionamenti contro i 4.930 richiesti (81\%). Le aziende Fiat interessate sono Iveco, Gilardini, Magneti Marelli e Geotech. (LaR)

Come si evince dalla prima parte della proposizione, la Fiat è un gruppo, al quale appartengono diverse aziende; alcune di esse sono elencate a seguire, in qualità di "aziende Fiat interessate" dai pensionamenti anticipati. Si noti che il nesso le aziende Fiat ricorre al plurale, ciò che non avviene, di regola, per gli usi con predicazione denominativa; a meno che il plurale non sia giustificato da più marchionimi coordinati:

(20) Detto fatto, i due [Riccardo e Cristina Carnovalini] vengono arruolati; i produttori di birra finanziano la loro spedizione; le ditte Lotto e Invicta li vestono e li calzano; la Ciemme e la Futura Assicurazioni procurano gli accompagnatori [...]. (LaR)

(21) Lo spettacolo è patrocinato dalla Regione Piemonte e dai Comuni di Asti e Alessandria; sponsor (hanno sostenuto gli oneri organizzativi e pubblicitari) le aziende Gancia e Oliviero. (LaR) 
Si ritorna, in questi casi, alla predicazione di tipo denominativo.

Nei suoi usi argomentali non denominativi, inoltre, il nesso può ricorrere introdotto da articolo indeterminativo, mentre con le predicazioni denominative ricorrono in genere articoli determinativi:

(22) Certo la Piaggio non è un'azienda Fiat essendo proprietà personale della madre di Giovannino, ma è chiaro che molti occhi a Torino guarderanno a Madrid per vedere come se la cava il ragazzo. (LaR)

Paradigmaticamente, a "la Piaggio non è un'azienda Fiat" corrisponde un nesso con aggettivo ("la Piaggio non è un'azienda torinese"). Piaggio, insomma, non appartiene al gruppo Fiat allo stesso titolo di altre; non è "un'azienda della Fiat". L'articolo indeterminativo non introduce qui un impiego "frazionante" del nome proprio (§1.1.1) bensì è indice del fatto che ci sono, appunto, diverse "aziende Fiat". Il nucleo del nesso nominale è il solo azienda, rispetto al quale il marchionimo ha funzione attributiva.

Il nesso aziende Olivetti ricorre, nell'esempio seguente, con un valore ancora diverso:

(23) La lettera, scritta ieri da impiegati, quadri e dirigenti, in poche ore è stata spontaneamente sottoscritta anche da oltre 4.000 operai, sui circa 7 mila che lavorano nelle aziende Olivetti della zona del Canavese, ad Ivrea. I firmatari sottolineano, tra l'altro, che se l'Olivetti "è stata una delle pochissime aziende al mondo che sia riuscita a passare con successo dalla meccanica all'elettronica e, quindi, all'informatica", lo si deve anche alle "capacità imprenditoriali" del presidente De Benedetti. (LaR)

Nella sua prima ricorrenza ("nelle aziende Olivetti"), azienda ha il valore concreto di stabilimento, di luogo fisico; più preciso sarebbe stato, in questo contesto, l'utilizzo di officine o fabbriche. Questo costrutto è pertanto diverso da "Le aziende Fiat" (19).

Ritorniamo ora al nesso "l'azienda (*della) Fiat" con valore di predicazione denominativa. Qual è, in questo caso, il valore del nome proprio entro il nesso nominale?

Nella letteratura sul tema, relativamente ai nomi propri prototipici, le discussioni ruotano attorno al concetto di "testa del sintagma nominale" - è il nome comune o il nome proprio? -, alla funzione dei due elementi e al rapporto tra loro (cf. tra gli altri Jonasson 1995; Kolde 
1995; La Fauci 2009). Strutture come il conte Monaldo o il professor Rossi sono usualmente definite come apposizioni interne (casi di apposizione semplice, che tende a precedere il nome proprio; cf. Cignetti 2010), in cui i nomi sono co-referenti. Si pone allora il problema di quale sia il nucleo del nesso nominale. ${ }^{5}$ La terminologia stessa tradisce la concezione del nome proprio come nucleo, al quale il nome che lo precede si aggiunge "per determinarlo e attribuirgli una proprietà particolare", 6 come avviene con un titolo professionale o nobiliare.

Jonasson (1995: 87) analizza il costrutto la camarade Catherine paragonabile al tipo la ditta Barilla con predicazione denominativa non come un unico sintagma formato da due nomi ma come la giustapposizione di due sintagmi nominali. Per la studiosa il nome proprio non ha funzione attributiva, bensì è una delle due teste di un sintagma nominale complesso. All'opposto, Vande Casteele (2012: 96) concepisce le "close appositive constructions" contenenti nomi d'impresa (per es. La compañia Puleva) come un solo sintagma nominale, con una testa - il nome proprio - e un modificatore avente funzione restrittiva (il nome comune, a sua volta eventualmente modificato da aggettivi o altro), senza che vi sia co-referenza tra i due componenti del sintagma.

Il secondo tipo, l'azienda (della) Fiat, è comparabile a le projet Delors nell'esempio di Kleiber (1985). Peculiarità della struttura, scrive Jonasson (1995: 87), sono la mancata co-referenza tra nome comune e nome proprio e il fatto che quest'ultimo è subordinato sintatticamente al primo, che è il nucleo del sintagma. Con i marchionimi, lo si è visto, tale subordinazione si manifesta nella possibilità di inserire la preposizione $d i$ davanti al nome proprio, possibilità che non è data per i costrutti in cui esso funge da predicazione denominativa: la ditta di Codemi e, con un antroponimo, il papa di Francesco non equivalgono ai corrispettivi senza preposizione.

La corrispondenza tra $N N m c$ e $N d i N m c$ non vige nel caso dei costrutti a carattere denominativo perché il legame tra nome comune e nome proprio non è di tipo subordinativo; vi è equivalenza (sintattica $\mathrm{e}$

5 Sulla difficoltà di individuare quale sia la testa e quale l'apposizione, in un nesso nominale costituito di due nomi di cui uno proprio, cf. $D L$ s.v. apposizione (G. Marotta).

6 La grammatica italiana (2012) s.v. Apposizioni (<www.treccani.it/enciclopedia/ apposizioni_(La_grammatica_italiana)/>, 7.11.2014). 
semantica) tra i due elementi, la quale rappresenta la condizione della possibilità, per entrambi i nomi, di ricorrere isolatamente con lo stesso valore (la Barilla = l'azienda). Piuttosto che parlare di apposizione interna per questi costrutti "azienda $N m c$ " si potrebbe allora, riprendendo la proposta di La Fauci (2009: 158), definire il marchionimo come una modificazione di tipo nominale, laddove il nome comune è in realtà il nucleo del nesso. In questo senso, ossia concependo il nome proprio come una modificazione, il suo valore è per certi versi simile a quello di un aggettivo; ma - lo si accennava sopra - lo è in maniera diversa rispetto ad altri tipi di costrutti $N c N m c$ per i quali è pensabile una sostituzione con un aggettivo vero e proprio (l'azienda fiatese o, come ricorre nel corpus LaR, "la ricetta benettoniana"), che il più delle volte non si realizza formalmente per motivi che non sono sintattici. Non è invece pensabile un costrutto "l'azienda benettoniana" per riferirsi a (la) Benetton in quanto azienda.

A chiusura del paragrafo, un'osservazione sulla frequenza del costrutto con modificazione denominativa. I casi citati all'inizio del $\$ 2.1 .1$ - l'azienda Mondadori, l'azienda Barilla ecc. - ricorrono nei dati in maniera sporadica; ben più numerosi sono i casi di la Mondadori e la Barilla. Nomi commerciali come Montano e Codemi, invece, paiono non ricorrere in funzione argomentale. Questo sarà da collegare non tanto a un'impossibilità sintattica, quanto a ragioni di enciclopedia condivisa: Montano e Codemi sono nomi di aziende meno note, per le quali la menzione di azienda o ditta è meno ridondante di quanto non lo sia per Barilla, se non addirittura necessaria alla comprensione. Quanto ai costrutti le aziende elettroniche Sony e Aiwa o l'impresa informatica Fujitsu, in essi è la presenza di un aggettivo modificante il nome comune a giustificare il ricorrere di quest'ultimo. Vi sono poi casi, come il brano citato a seguire, in cui il nome comune azienda è motivato da un parallelismo (e si noti la ricorrenza sinonimica di "alla Fiat"):

(24) Su per giù, tutti la pensano come l'ingegner Vittorio Ghidella che giorni fa, accompagnandomi in visita alla Fiat mi diceva: "Sì, l'azienda Fiat va bene, ma l'azienda Italia?". (LaR)

In molti degli esempi riportati, insomma, è possibile trovare una motivazione al ricorrere esplicito di azienda e consimili. Non in tutti, certo; 
del resto, la lingua sopporta molte ridondanze, anche quella per cui si può dire la ditta Barilla quando basterebbe (la) Barilla o l'auto Renault quando basterebbe la Renault (\$2.2.1). Nondimeno, si tratta di un numero ridotto di casi, mentre ben più numerose sono le ricorrenze di (Det) Nmc in cui, si può ipotizzare, il marchionimo stesso è sufficiente a manifestare il valore di "azienda" (cf. §1.1.2), perlopiù in combinazione con un determinatore $l a$ che costituisce variante libera. Qualora invece si aggiungano informazioni relative alla ditta cui allude il marchionimo, ecco comparire in maniera esplicita il nome comune: le aziende elettroniche Sony e Aiwa. Non potrebbe essere altrimenti, dato che "la Sony elettronica" farebbe propendere per una lettura "frazionante" del marchionimo, con aggettivo a valore restrittivo.

\subsubsection{Il costrutto marca/marchio Nmc}

Come azienda e ditta, pure marca e marchio ricorrono in combinazione con un nome commerciale. Si pone anche per il costrutto la marca Nmc la domanda relativa al rapporto tra nome comune e marchionimo, con la distinzione tra modificazione predicativa di tipo denominativo e di tipo relazionale. Vanno inoltre approfondite le differenze tra i costrutti la marca Nmc e il marchio Nmc. La combinazione marca/marchio Nmc, come si vedrà, ricorre essa stessa in funzione predicativa, presentando differenze formali e combinatorie che attendono ancora di essere indagate. A chiusura di due sezioni che hanno preso in considerazione le combinazioni con valore predicativo, sarà offerta infine una riflessione sul carattere metalinguistico di nomi quali marca e azienda, con le conseguenze che ciò comporta sul piano del nesso nominale.

(1) La marca Persol è stata valorizzata con l'apertura di numerose boutique nel mondo, le ultime delle quali a Beverly Hills e a Praga, che hanno consentito peraltro una maggiore penetrazione sui mercati americano ed europei. (LaR)

(2) La marca Lines invece è un esempio felice di storia patria. I famosi pannolini sono prodotti dalla Fater di Pescara, antica azienda chimica-farmaceutica che lega il suo successo al gruppo Angelini, che la rilevò negli anni '60. (LaR) 
(3) Poi, a metà degli anni Sessanta, la decisione di sospendere la produzione e la marca Invicta (che con l'azienda di zainetti non c'entra assolutamente nulla) finisce in una soffitta. (LaR)

(4) La marca Migros Sun Look è un po' più conveniente rispetto ai prodotti di marca, ma rimane sempre molto più cara della concorrenza germanica. (SM 2/05)

Questi nessi la marca Nmc presentano le proprietà combinatorie osservate nel \$2.1.1 per i costrutti l'azienda Nmc in cui il nome commerciale ha funzione denominativa. A la marca Persol corrisponde Persol è una marca, ciò che è indice della natura del marchionimo come predicazione nominale; parallelamente il costrutto con la preposizione $d i$, in cui il nome commerciale svolga una funzione manifestamente attributiva, non dà risultati pienamente equivalenti ('la marca di Invicta finisce in una soffitta). ${ }^{7}$

In alcuni brani si alternano la marca $N m c$ e la Nmc (cf. §2.1.1, ess. 12-13 per i nessi con azienda):

(5) In meno di un anno la marca Fiat ha perduto in Italia il 14 per cento, l'Alfa Romeo il 13 e la Lancia il 12, gli stranieri hanno conquistato saldamente la piazza italiana $[\ldots]$. (LaR)

(6) Nel 1992 la marca Pirelli ha realizzato nel settore delle due ruote un fatturato mondiale di circa 120 miliardi di lire, mentre quello della Metzeler Reifen è stato di circa 130 miliardi di lire. (LaR)

Là dove ricorre la marca Fiat (5) si può ipotizzare che, nei successivi l'Alfa Romeo e la Lancia, l'articolo abbia funzione di ripresa pronominale ('quella [scil. marca] Alfa Romeo') e l'uso nominale la Nmc veicoli il significato 'marca'. Resta peraltro sempre praticabile l'interpretazione di la Nmc come 'azienda'.

Un uso di la Nmc con valore 'marca' è inoltre possibile in proposizioni con copula in cui il marchionimo funge da soggetto (con o senza

7 Sembrano comportarsi diversamente i marchionimi a carattere spiccatamente propriale e antroponimico, come quelli relativi a stilisti: "Quanto vale il marchio Fiat? E il marchio Benetton? E quelli di Armani o Valentino?" (LaR). Forse perché, prima e più che "essere un marchio", Armani e Valentino "hanno un marchio", per cui quest'ultimo compare piuttosto come attributo in complementi preposizionali. 
determinazione) e si predica qualcosa a proposito della marca; quindi, in primo luogo, se ne predica l'essere una marca: ${ }^{8}$

(7) Dopo, quasi tutti i giocatori sono finiti a "Casa da Brahma", lo sponsor ufficiale brasiliano. La Brahma è una marca di birra: gli effetti di questo appoggio si sono fatti notare fin ai vertici federali, evidentemente. (LaR)

(8) La storia non si fa con tante piccole evoluzioni, ma con le grandi rivoluzioni. Fiat è una marca che forse non ha una tradizione estetica con stilemi costanti nel tempo e questo, invece di un'evoluzione costante, ci permette di innovare a grandi balzi. (LaR)

(9) La Lexus è una marca di automobili di lusso, esiste da soli dieci anni e vince ogni anno in modo spudorato tutte le classifiche di soddisfazione del cliente negli Stati Uniti $[\ldots]$. (LaR)

(10) La Daewoo, infatti, è una marca dall'immagine a basso profilo, ma vanta una solida esperienza industriale. (LaR)

(11) Della famosa pentarchia motociclistica italiana (Benelli, Bianchi, Gilera, Guzzi e Sertum) imperante negli anni Trenta, quando le due ruote rivestivano un ruolo davvero non secondario rispetto alle automobili, era proprio la Bianchi la marca più importante. (LaR)

(12) Niente Tods ai piedi, ma tante paia di scarpe Pollini. Moschino è invece la marca preferita per la maglieria. (LaR)

In questi costrutti emerge la natura di marca come classificatore, al confine tra lingua e metalingua. La marca Nmc appare come un nesso nominale in cui entrambi i nomi valgono da nucleo del nesso e in cui il marchionimo modifica marca come predicazione nominale, non diversamente da quanto osservato per la struttura l'azienda Nmc. Con la differenza, però, che in questo caso un uso argomentale del marchionimo(Det) $\mathrm{Nmc}$ - con valore equivalente è meno probabile. Laddove ricorresse la Nmc, in contesti comparabili a quelli citati, il costrutto sarebbe interpretato probabilmente come l'azienda Nmc per ragioni di luogo comune. Tale uso resta invece possibile, come visto negli esempi (5-6), quando la proposizione contiene un riferimento a marca correlato al marchionimo, che consente di interpretarlo, appunto, come marca.

8 Cf. l'es. di Van Langendonck (2007a: 97), "Ford is a familiar brand", discusso più avanti. 
Ci sono differenze nel funzionamento combinatorio del nesso $i l$ marchio $\mathrm{Nmc}$ rispetto a la marca Nmc?

(13) Prima la buona notizia. Il marchio Alfa Romeo c'è, e tiene ancora molto bene le sue posizioni nei gusti della clientela automobilistica medio alta. (LaR)

(14) "Il marchio Nivea, ben lungi dall'invecchiare, sta conoscendo una seconda giovinezza grazie a un nuovo prodotto che promette di eliminare i punti neri in due minuti", scrive L'Express. (LaR)

(15) Cominciammo, in seguito a una serie di indagini, a renderci conto che il marchio Barilla aveva qualche problema nei confronti dei possibili consumatori più giovani ai quali non diceva molte cose nuove. (LaR)

(16) Il marchio Benetton è presente in 120 Paesi, genera un fatturato di quasi 4 mila miliardi e garantisce 350 miliardi di utili all'anno. (LaR)

(17) Il marchio Wrangler sta per essere acquistato da Fiorucci. (LaR)

In questi esempi il marchio Nmc è comparabile ai nessi l'azienda Nmc e la marca $N m c$ : il nome commerciale vi funge cioè da predicazione di denominazione. Si nota che un uso nominale del marchionimo è qui ancora più raro (il Barilla?).

Marchio e marca, si è accennato nel paragrafo sulla terminologia (Parte I, §3.1), non sono sinonimi, benché ricorrano spesso in contesti proposizionali comparabili e presentino significati largamente sovrapponibili. L'analisi sintattica permette di portarne alla luce differenti caratteristiche combinatorie.

Innanzitutto, marchio ha - più di marca - il valore di 'logo', 'contrassegno grafico', correlato alla marchiatura visibile di un oggetto; con tale valore può ricorrere nei dati: ${ }^{9}$

9 In questo senso anche un nome proprio prototipico, per es. il nome di un personaggio noto, può essere un marchio: "La Fondazione si è trovata ad amministrare un patrimonio di 100 milioni di dollari, che ora cresce con il ricavato dell'asta, e si moltiplicherà grazie a una serie di spregiudicate iniziative commerciali per sfruttare il marchio Warhol: avremo l'orologio di Andy Warhol [...], il calendario di Andy Warhol [...]" (LaR). Warhol è un marchio senza essere una marca (in senso commerciale e giuridico). 
(18) [...] è necessario per tutte le grandi aziende cambiare periodicamente il 'logo' con il quale si è conosciuti, almeno fino a quando questo marchio non conosca una tale diffusione e un tale prestigio in tutto il mondo da essere inattaccabile. Il marchio Coca-Cola vale 35 miliardi di dollari, e non sarà mai cambiato. (LaR)

(19) Verdissimo il prato. Blu notte l'insegna bislunga venti metri, con l'ovale bianco e rosso del marchio Barilla da una parte: una gigantesca scatola di bucatini. $(\mathrm{LaR})$

(20) Come se non bastasse, la parte superiore dell'alettone, quella che si vede di più in tv, è interamente ricoperta dal marchio Marlboro mentre fino a due mesi fa c'era la scritta Goodyear. (LaR)

(21) Gli stilisti americani, stando al Wall Street Journal, starebbero progettando di sbarcare in Europa e in particolare in Francia: persino Chirac nel suo primo giorno di presidenza è stato fotografato vestito in stile Ivy League e con una camicia su cui era ben visibile il marchio Ralph Lauren. (LaR)

(22) [Luciano Benetton] È in giacca blu, pantaloni cachi, senza marchi Benetton addosso: "Non mi piace girare con il campionario", scherza [...]. (CoLFIS, periodico)

Diversamente rispetto ai casi di la marca $\mathrm{Nmc}$, in questi esempi il marchionimo non ha valore di predicazione nominale rispetto a marchio (che nell'es. 22 ricorre persino al plurale). È in gioco, d'altro canto, non il nome della marca quanto principalmente il suo valore di segno grafico, di logo. Inutile dire che una riduzione al solo marchionimo non pare possibile; è invece plausibile una corrispondenza con la struttura marchio di Nmc, caratteristica dell'uso del nome commerciale come predicazione non denominativa:

(23) Il marchio dell'Alitalia, con tutte le implicazioni d'immagine generale della compagnia di bandiera, per rimanere in Italia, è forse l'esempio più evidente della filosofia degli esperti ancorati nella baia di San Francisco. Ideato, come abbiamo detto, nel 1969, dopo 19 anni, è ancora attuale e competitivo ed è considerato tra i più eleganti simboli di compagnie aeree del mondo. (LaR)

Marchio allude a un elemento visibile: un "simbolo", come si legge nell'ultima parte della citazione. Non vi sono differenze combinatorie rispetto ai costrutti con altri nomi comuni, per es. $\operatorname{logo}$ (di) Nmc o dirigenti (di) Nmc, che si analizzeranno nel §2.2. Si può allora dedurre che marchio non è, qui, un classificatore. Quando tale nome comune 
non ha valore di classificatore, il marchionimo che lo segue introduce una modificazione attributiva, non denominativa, e funge da aggettivo relazionale.

Si registra poi un caso particolare:

(24) Ma anche Paolo [Gucci], il vendicatore solitario, non naviga in acque tranquille. Voleva creare una sua azienda, far nascere un nuovo marchio Gucci ma è stato bloccato dal pretore che ha giudicato illegittima questa iniziativa. (LaR)

(25) Quando Paolo [Gucci] ha tentato di presentare a Roma una sua collezione, s'è visto inibire l'uso del cognome dalla magistratura perché un nuovo marchio Gucci "avrebbe potuto far breccia sul pubblico a tutto danno dell'azienda di famiglia”. (LaR)

In questi brani è questione di un membro della famiglia Gucci, protagonista di annose battaglie legali dovute al tentativo di mettersi in proprio, creando una nuova griffe con il suo cognome. La creazione di "altri marchi Gucci", la potenziale moltiplicazione di enti definibili come marchio Gucci, che in quanto marchio non può prescindere dall'unicità, è stata ovviamente bloccata sul nascere per motivi tanto giuridici quanto (soprattutto) di profitto economico. L'articolo indeterminativo che ricorre negli esempi - altrimenti raro in questo tipo di struttura - si correla all'aggettivo nuovo relativo a marchio. Dalla considerazione di questa struttura nasce un caveat a non spargere troppi asterischi davanti a esempi fittizi (cf. Van Langendonck 2007a: 254-255 sui costrutti "This looks like a second Coca-Cola" e "another Ford [brand]").

Nei brani che seguono, invece, non si presentano situazioni che attentino all'unicità del marchio:

(26) La Philip Morris nicchia e per convincerla Buora propone a Dell'Utri le riprese 'mirate': "Si possono inserire nella sigla immagini della McLaren di Senna, o della Ferrari, sponsorizzate da Marlboro (altro marchio Philip Morris), o interviste a piloti che portino sulla tuta quel logo". (LaR)

(27) Prima dell'assalto dei giapponesi, un'automobile su due venduta negli Stati Uniti usciva dalle catene di montaggio della General Motors. Talmente intessuti nella vita quotidiana dei cittadini erano i suoi prodotti che i generali scelsero il motore della Cadillac - altro marchio $G M$ - per muovere il panzer prodotto negli Stati Uniti, lo Sherman. (LaR) 
Marlboro e Cadillac sono marchi che appartengono ad altri marchi, parallelamente al fenomeno studiato in precedenza ("azienda (della) Fiat" come "membro del gruppo Fiat'). Con "altro marchio Nmc" si intende non un marchio $\mathrm{Nmc}$ diverso da quello cui solitamente ci si riferisce (come sarebbe stato per Gucci se l'impresa di Paolo Gucci fosse andata in porto), bensì un ulteriore marchio incluso nella sfera giuridica ed economica di Philip Morris o GM. Mentre nel caso di Gucci l'inserimento della preposizione di cambia il significato del costrutto (perché un nuovo marchio di Gucci implicherebbe l'appartenenza di tale marchio $a$ Gucci, ciò che appunto non vale in questo contesto), negli esempi (26-27) tale equivalenza è possibile ("altro marchio di Philip Morris"). Di conseguenza, il marchionimo funge in essi da aggettivo relazionale.

Negli esempi sin qui analizzati il nesso marca/marchio $\mathrm{Nmc}$ svolge sempre funzione argomentale e ricorre con determinatore (con l'eccezione degli ess. 26-27, in cui costituisce un inciso). Ma la combinazione marca/marchio $\mathrm{Nmc}$, non determinata, ricorre pure in funzione attributiva:

(28) Paul McCartney ha giurato che non si raderà più con un rasoio di marca Gillette fino a quando la società che fabbrica le famose lamette non smetterà di usare cavie animali per collaudare i propri prodotti. L'ex Beatles ha rispedito al mittente il suo rasoio, la schiuma da barba e tutti gli altri prodotti marca Gillette. (LaR)

Il nome comune marca seguito dal marchionimo Gillette modifica predicativamente un altro nome comune (rasoio, prodotti).

È auspicabile operare una differenziazione tra questo tipo di costrutto, (di) marca Nmc, non infrequente nei dati analizzati, e la struttura Det marca Nmc. La differenza, in termini combinatorî, è chiara: quest'ultimo costrutto è un nesso nominale, necessariamente determinato, che può svolgere funzione di argomento nella proposizione. Il costrutto evidenziato in (28), invece, non è un nesso nominale autonomo; ricopre funzione attributiva rispetto a un altro nome, riferito perlopiù a un prodotto.

Ecco altri esempi di questo tipo, con preposizione di:

(29) Corso Marconi, che ieri ha annunciato tra l'altro un aumento dell' 1,5 per cento dei prezzi delle auto di marca Fiat a partire dal primo giugno, preferisce non scoprire ancora le carte. (LaR) 
(30) Da poco sono terminate le verifiche su tutte le pistole di marca Beretta calibro 22 che si trovano in provincia di Firenze. (LaR)

(31) "Il ragno d'oro", nuovo romanzo di Giuseppe Pederiali di prossima pubblicazione da Rizzoli verrà consegnato ai librai con un chilo di tortellini di marca Fini ogni venti copie ordinate. (LaR)

(32) Tra le poche concessioni che [Valentino Zeichen] si è permesso, ma per ragioni sanitarie, ci sono i sandali altrettanto leggendari che porta otto mesi l'anno. Ma sono sandali che ora non si fanno più, di marca Bally, che andava a comprare in Svizzera con gli ultimi soldi rimasti [...]. (LaR)

(33) Lo "Yamaha Award" è stato conferito ai pneumatici per motociclette sia di marca Pirelli sia di marca Metzeler (prodotti dalla consociata tedesca Metzeler Reifen). (LaR)

Nel nesso nominale di marca Nmc, marchionimo e nome comune non hanno lo stesso statuto, tant'è vero che il marchionimo non può essere tralasciato. Questo vale anche per i casi in cui la struttura è marca $\mathrm{Nmc}$, senza preposizione: ${ }^{10}$

(34) Di pistole calibro 22 marca Beretta, l'arma che solitamente usa l'assassino, a Firenze ce ne sono 14 mila. Le stiamo controllando tutte. (LaR)

(35) Ancora un viaggio negli amati-odiati spot per l'ineffabile Federico. Dopo i brividi vagamente equivoci evocati dai "Rigatoni" marca Barilla in bocca a una bella e famelica signora, [...] Fellini torna alla pubblicità con una mini-serie onirica che promette di far storia. (LaR)

(36) Quale madre, di fronte al figlioletto teneramente abbracciato a un lupacchiotto di peluche marca Trudi dagli occhi imploranti oserà ancora dire: "Sta buono, altrimenti chiamo il lupo?". (LaR)

(37) Il ragazzo era con il suo motorino, un'Aprilia, e indossava un giubbotto rosso marca Dainese, con sotto una felpa blu e un paio di blue jeans. (LaR)

(38) È un orologio marca Breil con il cinturino azzurro l'ultima frontiera della difesa di Massimo Foglia. (LaR)

(39) Per corrompere i soldati ai posti di blocco jugoslavi meglio portarsi appresso sigarette marca Marlboro o, come ripiego, le Rothmans. (LaR)

10 Si formula l'ipotesi, che andrebbe verificata in corpora recenti, che $N$ marca Nmc sia un costrutto datato. 
Marca Nmc funge da aggettivo composto, tanto che può essere seguito da un altro aggettivo riferito al nome comune:

(40) L'allarme "bionde atomiche", dopo ventiquattro ore, è un giallo internazionale sempre più complicato. Del carico di "sigarette di contrabbando marca Marlboro radioattive, proveniente da depositi russi contaminati" denunciato direttamente ai giornali dal sottosegretario alla sanità Savino, non c'è traccia. (LaR)

La combinazione di marca e nome commerciale equivale all'uso del solo nome commerciale in funzione predicativa: a "sigarette marca Marlboro" corrisponde "sigarette Marlboro". Un altro costrutto parallelo è Det Nmc in riferimento al prodotto: in (39), "sigarette marca Marlboro" è accostato a "le Rothmans".

La struttura attributiva (di) marca Nmc non ricorre con marchio. ${ }^{11}$ Con quest'ultimo compare però la versione con preposizione con e articolo:

(41) [...] in pochi anni potrebbero arrivare in America non solo milioni di televisioni Sony o di auto Honda, ma anche aerei civili con il marchio Mitsubishi. (LaR)

In questo caso è lecito ipotizzare che la menzione esplicita di Mitsubishi come marchio, invece di utilizzare semplicemente il marchionimo in funzione attributiva, sia dovuta al fatto che esso non si correla agli aerei quanto a conoscenze enciclopediche, come invece Sony a prodotti dell'elettronica e Honda alle automobili, per cui aerei Mitsubishi sarebbe forse risultato anomalo.

La struttura (N) con il marchio Nmc ricorre anche in altri contesti:

(42) Tutto questo sarà ora riconsegnato al genio imprenditoriale di Bata. Si tratta solo di sapere se gli verrà affidato per intero un solo stabilimento che produca scarpe col marchio Bata o si cercherà di coinvolgerlo nella gestione di tutto il settore. (LaR)

(43) Sul piatto della bilancia interessi non certo piccoli visto che Paolo [Gucci] intendeva mettersi in proprio, firmare altre borse, foulard, scarpe con il marchio Gucci spezzando così un monopolio e giganteschi interessi. (LaR) ${ }^{12}$

11 Forse perché, come accennato sopra, marchio ha più che altro valore di segno grafico.

12 L'esempio fa il paio con quelli riportati più in alto (24-25): stavolta il marchio Gucci è un nesso determinato, con riferimento all'unico "marchio Gucci" che 
(44) Dopo la seconda guerra mondiale la Nestlé acquista Alimentana, che produce e vende con il marchio Maggi. (LaR)

In questi esempi è un predicato verbale - firmare, produrre, vendere - a reggere il nome comune o, in (44), direttamente il nesso preposizionale. La struttura ricorre anche senza articolo (con marchio $\mathrm{Nmc}$ ):

(45) Si prevede una produzione di 320 mila vetture l'anno con marchio Alfa, contro le attuali 190 mila. (LaR)

(46) Seguendo queste nuove tendenze, la Coop ha annunciato a breve l'apertura di nuovi ipermercati (si parla di 400) che venderanno solo prodotti con marchio Coop. (LaR)

(47) Infine non va dimenticata la sponsorizzazione degli orologi con marchio Marlboro. (LaR)

Non si osservano variazioni sostanziali rispetto al nesso con preposizione articolata.

Si può affermare lo stesso anche per i costrutti della marca Nmc in confronto a quelli visti sinora, di marca $N m c$, che non presentavano determinazione?

(48) Da questa mattina, come era nelle previsioni del gruppo automobilistico di Torino, i prezzi delle vetture della marca Fiat aumenteranno del 3 per cento. $(\mathrm{LaR})$

(49) I gorilla di Koskotas hanno raccontato di averli consegnati a Luvaris in banconote di piccolo taglio stipate in un grande scatolo [sic] di cartone di pannolini per neonato della marca Pampers. (LaR)

In questi esempi, della marca Nmc compare come modificatore di un nome di prodotto (vetture, pannolini per neonato) e non presenta variazioni essenziali rispetto a di marca Nmc.

Quando la struttura modifica nomi astratti, invece, emergono delle differenze:

esiste e può legalmente esistere, ma il problema consiste nel fatto che tale marchio, nelle intenzioni di Paolo Gucci, avrebbe firmato "altre borse" ecc., ossia prodotti diversi da quelli che, per diritto, possono recare il marchio. 
(50) In Europa l'incremento della marca Fiat è stato del 12,7 per cento (+135 mila vetture). ( $\mathrm{LaR})$

(51) Il risultato più ghiotto per la Fiat è sicuramente rappresentato dall'andamento commerciale specialmente del settore automobilistico. Vediamo qualche cifra. Nei primi cinque mesi sono state vendute 930 mila vetture di cui 85 mila con il marchio Alfa. Nell' 86 il bilancio dei primi cinque mesi non era andato oltre le 750 mila vetture esclusa la quota Alfa Romeo. Dunque c'è stato un forte incremento di marca Fiat accompagnato da un leggero miglioramento dell'Alfa: il che è sorprendente tenuto conto che ancora l'Alfa opera con un prodotto tutto da rinnovare. (LaR)

In (50) il nesso ha valore di genitivo soggettivo: "l'incremento della marca Fiat" equivale a "la marca Fiat ha un incremento" (cf. infra, §3.2.1). In (51), "c'è stato un forte incremento di marca Fiat", non può essere così, perché marca, non determinato, non può ricorrere in funzione argomentale ('marca Fiat ha avuto un incremento). Questo secondo costrutto si avvicina al tipo targato/firmato $N m c$, che sarà approfondito più avanti (§4.5). La marca che viene applicata, in funzione predicativa, a un nome astratto come incremento non è, chiaramente, una "marca" in senso giuridico o economico. Così pure negli esempi che seguono:

(52) Callieri ha seguito, gradino dopo gradino, una brillante carriera: tutta di marca Fiat, anche quando ha lasciato Corso Marconi per andare alla Gilardini e poi al gruppo Rizzoli-Corsera. (LaR)

(53) Ieri sono stati raggiunti da ordine di custodia cautelare [...] l'ex segretario provinciale del Psi Alessandro Perelli (già arrestato nell'ambito dalla magistratura di Milano per una tangente di marca Fiat) [...]. (LaR)

(54) La verità è che i guai più grossi sono tutti di marca Fiat. (LaR)

(55) Secondo: la pole position di marca Ferrari. Che si è ripetuta ieri. (LaR)

(56) "Vorrà dire che Schimberni andrà all'inferno, ma insieme alla Fondiaria" dice un signore, sorriso ammiccante e sguardo un po' perso, in completo blu scuro, quasi a lanciare un messaggio di marca Montedison alle frotte di giornalisti, fotografi e cineoperatori assiepati davanti al portone di Foro Bonaparte. (LaR)

Non si può dire che carriere, tangenti e così via "abbiano una marca" come gli spaghetti o le automobili; in questi contesti, di marca Nmc è 
utilizzato in maniera traslata. Del resto, tale struttura è diffusa anche con antroponimi che non sono marche:

Buttiglione gli risponde che si dimetterà al congresso, semmai, ma intanto è già pronta la mozione di sfiducia firmata Fitto e Bartolozzi, appunto, i due vicesegretari dimissionari di marca Formigoni. (LaR)

In sintesi, tra le strutture contenenti marca/marchio si distinguono le modificazioni predicative - il tipo (di) marca Nmc, ma anche con (il) marchio Nmc - e quelle che costituiscono un nesso nominale determinato (Det marca/marchio $\mathrm{Nmc}$ ). In queste ultime, il marchionimo funge da modificatore denominativo del nome comune (come ne l'azienda $\mathrm{Nmc}$ ) nei casi in cui non può essere preceduto dalla preposizione $d i$.

Si chiude ora il paragrafo con alcune riflessioni generali sui nessi in cui ricorre il nome comune marca.

Nella Parte II (cap. 2) si è menzionata la prospettiva di Van Langendonck (2007a: 236) sui nomi commerciali. Sulla base di cinque esempi che contengono il marchionimo Ford, lo studioso identifica cinque diverse categorie di referenti dello stesso: fondatori di imprese, le imprese medesime, marche (brand), prodotti della marca e singoli esemplari del prodotto. Si propone ora un approfondimento sulla terza categoria: il marchionimo come brand. Un paradosso, un'ovvietà o una formulazione poco convincente?

Se è di potenziali portatori di tale nome che si sta parlando, allora la lista di categorie è ampliabile in modo indeterminato: Ford è un consiglio d'amministrazione, un titolo in borsa, uno stile di vita e così via, così come Andrea Doria è un ammiraglio genovese, una corazzata, una società sportiva. Se invece, come si deduce dagli esempi, l'obiettivo è trovare costrutti in cui il nome Ford, usato in funzione argomentale, individua referenti diversi, allora l'esempio addotto dallo studioso "Ford is a familiar brand" - è inadatto in quanto si tratta di un costrutto predicativo in cui si afferma che Ford è, appunto, una marca (cf. gli ess. $7-12$ in questo paragrafo).

In apparenza il costrutto allinea il significato Ford = 'marca' agli altri (patronimico, impresa, prodotto). Da un punto di vista sintattico, però, esso contribuisce a celare un'importante differenza, emersa nell'analisi: mentre negli altri casi un uso argomentale del marchionimo, con 
un valore specifico, è plausibile, il nome Ford difficilmente ricorrerà con il significato di 'marca'. Nell'esempio di Van Langendonck, insomma, l'uso nominale di Ford = 'marca' sembra reso possibile soltanto dalla struttura predicativa dell'esempio. Sostenere che Ford è riferito (anche) a una marca perché compare nella proposizione "Ford è una marca conosciuta" è come dire che Giulia è un nome di persona perché ricorre nel costrutto "Giulia è un nome diffuso". Vi è una sorta di tautologia, combinata a una ricorrenza quasi metalinguistica del marchionimo (cf. Janner 2013: 252n).

È chiaro - e in questo Van Langendonck ha ragione - che Ford è una marca, come pure un'impresa e un prodotto (automobile). ${ }^{13}$ Ford riferito all'impresa o a un prodotto della stessa ricorre però anche in contesti in cui i nomi impresa e automobile non compaiono affatto: " $\mathrm{La}$ Ford vuole il 'placet' del sindacato italiano" (LaR); "due altri terroristi aspettavano Bienzobas su una Ford" (LaR). Il valore di marca, invece, si manifesta perlopiù in usi attributivi, nella fattispecie (Det) marca Nmc. In altre parole, nel nesso la marca Nmc non c'è lo stesso grado di ridondanza che c'è ne l'azienda Nmc. Pur essendo chiaro che Ford è una marca, questa informazione non è ritenuta implicita nel nome proprio stesso.

Si potrebbe obiettare che nei costrutti ( $L a) N m c$ è in grande crescita, (La) Nmc ha venduto più dell'anno scorso (usi argomentali: $\S 1.1 .2$ ) vi può essere un riferimento alla marca e non all'azienda, ipotesi che sarebbe anch'essa legittimata dal genere e numero del nome comune implicato. Nondimeno, questo significherebbe attribuire a marca il tratto [+ umano] (si pensi ai casi, reperiti nei corpora, di "Tefal propone un apparecchio molto funzionale" o "Migros ha deciso di ampliare il proprio assortimento"). Legittimato metonimicamente nel caso di $N m c$ = 'impresa' (chi vi lavora sono esseri umani), il tratto [+ umano] è attribuibile alla marca solo indirettamente. Ma, in questi termini, riferire (la) Nmc alla marca sembra poco economico, quando il significato di azienda è così alla mano. Nonostante i tentativi del marketing di umanizzare la marca, essa rimane non-umana, perlomeno da un punto di vista linguistico.

13 Sarebbe interessante studiare quale di questi valori di Ford emerga per primo nella coscienza dei parlanti. 


\subsection{Marchionimo come modificazione attributiva}

Si rivolge ora l'attenzione alla classe di costrutti $N \mathrm{Nmc}$ in cui il nome commerciale non rappresenta una predicazione denominativa (' $N$ chiamato $N m c^{\prime}$ ), bensì un semplice attributo.

Gli esempi del \$2.2.1 contengono nessi in cui tra nome comune e marchionimo si riconosce un rapporto di tipo "prodotto-produttore". Può esservi coincidenza tra questi costrutti $N N m c$ e gli usi argomentali del marchionimo (Det Nmc). Ciò non si riscontra per i nessi del $\S 2.2 .2$, in cui ricorrono perlopiù nomi astratti; tra i due componenti del costrutto vige, a seconda dei casi, un rapporto di tipo possessivo e/o soggettivo. Il marchionimo non modifica il nome comune in termini denominativi; si tratta peraltro di entità solitamente "non nominabili" come risposta (Fiat), obiettivo (Fiat). Al contrario, i nessi studiati nel $\S 2.2 .3$ presentano nomi umani, quindi nominabili, ma - diversamente dalle strutture $N \mathrm{Npr}$ contenenti antroponimi - i nomi commerciali non ne costituiscono un modificatore denominativo.

\subsubsection{Nessi N Nmc con nomi concreti (relazione prodotto-produttore)}

Frequenti sono i casi in cui il nome commerciale funge da aggettivo qualificativo apposto a un nome concreto relativo a un prodotto:

(1) La Procter \& Gamble è un colosso americano presente in Italia con una infinità di marchi: dai detersivi per la casa, ai farmaceutici, al caffè. Produce fra gli altri Dash, Ariel, Mastrolindo, ma anche la crema antibrufoli Clerasil [sic], le caramelle Victors, il sapone Camay, il dentifricio AZ e il caffè Splendid. E appunto $i$ pannolini Pampers. (LaR)

Come una sorta di etichetta (cf. Folena 1964: 119) di carattere predicativo, il marchionimo apposto al nome di prodotto lo identifica con la sua denominazione commerciale, distinguendo "le caramelle Victors", "il dentifricio AZ", "i pannolini Pampers" da innumerevoli altri tipi di pannolini, dentifrici e caramelle. L'utilizzo aggettivale del nome commerciale, d'altra parte, è quello raccomandato da esperti di marketing e diritto, come ricorda Vaxelaire (2005: 217). 
Studiando le strutture in cui si combinano nome comune e nome proprio, Jonasson (1994: 107-108) osserva che, nei casi in cui il nome comune designa un oggetto concreto, la funzione pragmatica più pertinente pare essere "X è l'ideatore/il fabbricante di Y". Questo si può applicare a una larga parte dei costrutti presentati in questo paragrafo: per il costrutto i pannolini Pampers si avrà 'Pampers produce (de)i pannolini'. Il marchionimo ha dunque funzione attributiva e non funge da predicazione denominativa.

Con alcune eccezioni: una parte dei nessi che ricorrono in (1) contiene un nome attribuibile non a un'impresa ma a un prodotto specifico. Il sapone Camay non è 'il sapone prodotto da Camay', bensì 'il sapone chiamato Camay' o 'con il marchio Camay'. Il costrutto si avvicina allora alla struttura con predicazione denominativa $(\S 2.1)$.

vi spiego perché perché eh Alleluja [in realtà Alleluya] in modo particolare per dire ma in genere tutti un po' tutti tutti gli yogurt della Yomo sono controllatissimi da parte degli esperti [...] ci sono tanti gusti dentro a questo yogurt Alleluja che sono ispirati dalle vecchie ricette dei pasticceri di un tempo (LIP MD 18)

Alleluya, come si evince dall'inizio di questo brano (un inserto pubblicitario), è un tipo di "yogurt della Yomo". In quanto nome attribuito a un prodotto specifico - con un atto di battesimo, nei termini di Kripke (1972) -, Alleluya è il nome proprio di tale prodotto, quindi nella struttura yogurt Alleluya esso rappresenta un predicato di denominazione; si potrebbe affermare, con un costrutto predicativo, che 'Alleluya è uno yogurt'.

In una prospettiva sintattica, Yomo funziona in modo diverso, pur ricorrendo anch'esso nel costrutto yogurt Nmc: accanto a yogurt Alleluya si ha per es. "un riuscitissimo spot dello yogurt Yomo" (LaR). La differenza, dal punto di vista combinatorio, risiede nella possibilità di una struttura yogurt della Yomo (che ricorre altrove nel testo citato), equivalente a yogurt Yomo; nel caso di Alleluya non c'è alternanza con il complemento di specificazione ('yogurt dell'Alleluya), perché tra i due membri del nesso non c'è un rapporto di dipendenza come tra yogurt e Yomo. Pertanto, i due costrutti superficialmente equivalenti yogurt Yomo e yogurt Alleluja celano in realtà, dal profilo funzionale, relazioni diverse tra i due elementi. Essendo coinvolti nomi propri, vi è in entrambi i costrutti, a qualche livello, una predicazione metalinguistica: 
in un caso, però, essa è diretta (yogurt Alleluja = 'yogurt che ha nome Alleluja'), nell'altro soltanto indiretta (yogurt Yomo = 'yogurt prodotto da un'azienda/con un marchio che ha nome Yomo'). ${ }^{14}$

Se si eccettua questa tipologia di costrutti - funzionano allo stesso modo anche "i cioccolatini Ferrero Rocher, Pocket coffee e Mon chéri, gli snack al cioccolato Duplo e Tronky" (CoLFIS, periodico) -, per il resto la struttura con nome di prodotto e marchionimo si inserisce nel tipo attributivo. Eccone alcuni esempi (l'elenco è ampliabile a piacimento):

(3) Dopo la data di scadenza, le bottiglie Soda-Club possono esplodere. (SM 2/10)

(4) Le scarpe Repetto, tra le più care, non soddisfano: «La suola in cuoio fine non durerà molto. Queste scarpe andrebbero portate per stare in casa». (SM 3/07)

(5) Unanimità c'è stata anche sui prodotti poco appetitosi: i bastoncini di merluzzo Coop e quelli croccanti di nasello Migros sono finiti in fondo alla graduatoria. (SM 4/04)

(6) Spaghetti mon amour. È Gérard Depardieu il "testimonial" della pasta Barilla in Francia. (CoLFIS, periodico)

(7) [...] la bottiglia è già diventata un oggetto da collezione per la sua rarità. Proprio come gli orologi Swatch, che da vero e proprio fenomeno sociale sono oggetto di speculazione da parte dei collezionisti. (CoLFIS, periodico)

(8) quell'annuncio che aveva messo su Seconda mano relativo a quella moto Yamaha per favore mi dovrebbe richiamare visto che lei ha la segreteria telefonica (LIP MB 87)

(9) sì buongiorno senta io ho portato la settimana scorsa un registratore Philips a riparare e volevo sapere se era pronto (LIP FB 20)

La funzione attributiva, lo si è ricordato, si lega alla possibilità di utilizzare il nome commerciale anche come complemento di specificazione (cf. Jonasson 1994: 107; Vaxelaire 2005: 212-213): "la pasta della Barilla", "un registratore di Philips" e così via. In virtù dell'equivalenza con il costrutto preposizionale è lecito affermare che il marchionimo funziona come un aggettivo relazionale, alla stregua di una ragazza milanese/una ragazza di Milano (La Fauci 2009: 154).

14 Stabilire se il marchionimo sia qui nome d'impresa o di marchio è difficile e comunque non necessario. 
La possibilità di stabilire equivalenze tra costrutti formalmente simili non è, si noti, un uzzolo dei linguisti di stampo distribuzionalista ma una prospettiva metodologica che prende atto di una peculiarità della lingua effettivamente ricorrente nel discorso:

(10) Bando assoluto degli sponsor dalle gare (e c'è un'inchiesta in corso per scoprire chi ha permesso alle staffettiste della $4 \times 400$ di salire sul podio con una tuta Adidas). (LaR)

(11) In piedi di fronte alla sua camera d'ospedale, vestito in una tuta della Adidas, sorridente e apparentemente in piena forma, [Boris Eltsin] ha concesso un'intervista al tg Vremja [...]. (LaR)

Alternanze tra i due costrutti si riscontrano persino nella medesima proposizione:

(12) Sull'imballaggio del modello Mivit ci sono le istruzioni, con quello di Zyliss bisogna arrivarci da soli. (SM 3/07)

Alla ripresa pronominale (quello = 'modello'; v. §1.1.4) si accompagna un nome commerciale introdotto da preposizione.

I nomi comuni modificati da un marchionimo in funzione attributiva, come si vede in quest'ultimo esempio, fanno riferimento non solo a prodotti specifici ma anche, genericamente, a "modelli", "prodotti" o "impianti":

(13) Nel 1933 Guidotti ebbe la responsabilità del collaudo di tutti i modelli Alfa Romeo, da turismo e da corsa. (CoLFIS, quotidiano)

(14) I prodotti Nike sono diventati sinonimo di schiavitù, abusi, lavori forzati. (LaR)

(15) Se uno è sorteggiato e chiamato per telefono da Raffaella, vince un milione per ogni prodotto Buitoni che ha in casa. (LaR)

(16) L'impianto Sony che ha vinto il test non può essere messo in standby, ma passa automaticamente allo stato eco quando lo si spegne. (SM 6/07)

L'intera struttura $N N m c$ può essere modificata da attributi, sia aggettivi, sia altri elementi marchionimici, accordati al nome comune ove possibile (17): 
(17) [Gianni P.] Gira fra le vie del rione Carrassi, con puntate in altri quartieri e frazioni di Bari, come Carbonara, a bordo di una moto Aprilia bianca e rosso fuoco, troppo grande per lui. (CoLFIS, quotidiano)

(18) Forse quelle immagini hanno ricordato a Pietro Barilla, che per me è un amico, un'altra stagione: l'Urss che vide da soldato semplice, autista alla guida di un camion Lancia $3 \mathrm{Ro}$, che con 40 gradi sottozero trasportava truppe sconsolate verso il fronte [...]. (CoLFIS, periodico)

\section{Si riprenda ora il brano pubblicitario già citato:}

(19) e poi c'è lo yogurt Alleluja [...] vi spiego perché perché eh Alleluja in modo particolare per dire ma in genere tutti un po' tutti tutti gli yogurt della Yomo sono controllatissimi da parte degli esperti [...] e io so ecco perché avevo cominciato a parlare dello yogurt Alleluja perché io ho riscontrato che soprattutto tra i bambini quello che è favorito è l'Alleluja ma perché Alleluja * forse perché l'Alleluja sa un po' di pasticceria * perché ci sono tanti gusti dentro a questo yogurt Alleluja che sono ispirati dalle vecchie ricette dei pasticceri di un tempo [...] l'altra settimana abbiamo parlato di quello alla frutta oggi abbiamo parlato dell'Alleluja quindi domani donne quando andate al supermercato prendete tutti i gusti (LIP MD 18)

La struttura $N N m c$ alterna con l'uso argomentale del marchionimo, in parte determinato. Da etichetta attributiva, il nome commerciale passa all'utilizzo come nome: (l')Alleluya per 'lo yogurt Alleluya'.

L'uso argomentale del nome commerciale si presenta in (19) come equivalente del costrutto predicativo. È una situazione diffusa, tanto con nomi cosiddetti di prodotto quanto - ancor più evidente - con nomi d'impresa, utilizzati metonimicamente con riferimento a un prodotto dell'impresa stessa: ${ }^{15}$

(20) L'acqua minerale Valser, una delle più costose, secondo la sua pubblicità, «fa bene alle ossa» perché contiene tanto calcio. [...] Eppure non è necessario spendere così tanto per un'acqua ricca di calcio. Lo dimostra la marca propria di Denner proveniente dalle Alpi Bernesi: con 37 centesimi al litro, quest'acqua costa meno della metà della Valser e contiene il $27 \%$ di calcio in più. (SM 6/05)

(21) Due pannolini sono invece stati giudicati solo sufficienti, gli M-Budget e i Delora. [...] Migros fa notare che i pannolini M-Budget devono per forza avere alcune differenze, dovute alla differenza di prezzo, in confronto a Milette.

15 Presentano proprietà simili, inoltre, i nessi del tipo azione $N m c$, in particolare al plurale (le Fiat = 'le azioni Fiat'; cf. §1.2.1, ess. 21-24). 
Sottolinea però che «un pannolino M-Budget copre comunque ancora bene $\mathrm{i}$ bisogni basilari di un bebè». (SM 5/10)

(22) E sempre ieri pomeriggio, negli uffici del Nucleo Operativo di via Valfrè, è stato sentito anche l'amico che prestava abitualmente il Suzuki Vitara nero a Gianni. Il fuoristrada che sembrava scomparso era invece regolarmente parcheggiato in una strada a Barriera di Milano. [...] Poi sono arrivati due ispettori di polizia. Hanno registrato le targhe delle vetture sospette. "Compreso il fuoristrada Suzuki Vitara". (CoLFIS, quotidiano)

Questi brani offrono esempi di variazione tra uso predicativo e uso argomentale dello stesso nome commerciale: l'acqua minerale Valser e la Valser (20), gli M-Budget e i pannolini M-Budget (21), il Suzuki Vitara e il fuoristrada Suzuki Vitara (22), ${ }^{16}$ in un'alternanza che può essere giustificata a livello testuale da esigenze di variatio.

Si noti che la possibilità di utilizzare nominalmente marchionimi che fungono da attributi è sfruttata anche quando nel contesto immediato non è reperibile un nesso $N N m c$ equivalente:

(23) Cosa vuole, non dico che un arbitro debba per forza mettersi in giacca e cravatta, ma uno che si presenta con gli stivaletti a tacco alto, i Ray-Ban, l'orecchino al lobo, il codino di 40 centimetri, lei mi capisce... (LaR)

(24) "Preferire lo shopping ad altre attività mi sembra una mancanza di fantasia spiega Diego Luparelli, psicoterapeuta - la gratificazione che può dare lo shopping è di breve durata, è una forma di piacere a breve termine, non è che tutti $\mathrm{i}$ giorni si può comprare una Lacoste". (CORIS STAMPAQuotidiani)

(25) Nella mattinata di ieri i familiari di Giovanni Falcone hanno consegnato soltanto il Toshiba portatile e il data bank Casio che, abitualmente utilizzati da Falcone, non figuravano nell'inventario dei reperti sequestrati a Roma e Palermo. Ma con il Toshiba e il Casio i familiari non hanno potuto consegnare ciò che non hanno trovato e non potevano trovare, ovvero gli "appunti" del giudice assassinato. (LaR)

(26) Castel d'Agogna, paese non molto distante da Mortara, è il decimo centro della Lomellina ad alzar bandiera bianca: vietato bere e cucinare con l'acqua che sgorga dai rubinetti. Gli spaghetti cuoceteli con la Ferrarelle. (LaR)

16 Cf. anche: "Ecco alcune guide-novità da mettere nella valigia prima di partire. Guide Rosse Michelin. [...] Per la Francia, gli aggiornamenti alla celebre Michelin di cui i viaggiatori non sanno fare a meno" (CoLFIS, periodico). 
Questi usi argomentali di marchionimi - i Ray-Ban, una Lacoste, il Toshiba, la Ferrarelle - possono corrispondere, tra gli altri, ai costrutti $N N m c$ dei brani seguenti:

(27) Gli occhiali Ray Ban da aviatore: cinquantamila a Washington, a Roma almeno il doppio. (CoLFIS)

(28) "Perché stupirsi? Questa è una mutanda decorosa, fresca. Per me è come un jeans, una maglietta Lacoste [...]" spiega Karl Lagerfeld, assediato dai fotografi quanto Claudia Schiffer. (CoLFIS, quotidiano)

(29) Il deficit deriva soprattutto dalla abilità tecnica e commerciale dei Giapponesi, e dalla passione americana per le auto Honda, i computer Toshiba, le televisioni Sony e i videoregistratori Panasonic. (CoLFIS)

(30) Il vero fondatore di Azzurra e dell'avventura italiana in Coppa America fu Mario Violati, uno dei Violati proprietari dell'acqua minerale Ferrarelle. (CoLFIS)

Chiaramente non si può stabilire un rapporto biunivoco tra le coppie di costrutti. Per una Lacoste è pensabile pure un'equivalenza con una polo Lacoste. Il punto non è la ricerca di un costrutto "integrale" dal quale deriverebbe il nesso "ridotto", bensì lo stabilire equivalenze funzionali tra costrutti nei quali il nome commerciale ricorre con funzioni diverse, in particolare quelle di predicato e argomento. A questo proposito si richiama l'ipotesi elaborata nel $\S 1.1 .4$ a proposito dei costrutti Det $N m c$, ovvero che si tratti di attributi nominalizzati (in maniera non dissimile da quanto avviene con gli aggettivi), di predicati divenuti argomenti.

Si è affermato che i costrutti la pasta Barilla o l'acqua minerale Ferrarelle non contengono predicati di denominazione (anche perché c'è coincidenza con il tipo la pasta della Barilla). Quindi, coerentemente con quanto affermato, si avrà Barilla è un marchio/un'azienda ma non Barilla è una pasta. Bisogna però aggiungere che, raggiunto un determinato grado di diffusione del costrutto nominale, un nesso come (La) Barilla è una pasta può ricorrere. L'attributo, insomma, può essere nominalizzato.

In questi nessi il marchionimo è retto dal nome comune, nucleo del costrutto. A proposito di costrutti simili, quali mattonelle Minori, Migliorini (1927: 341) parla di "giustapposizioni” esprimenti 
dipendenza. ${ }^{17}$ Questo implica che siano necessarie condizioni particolari perché la predicazione attributiva si nominalizzi; detto altrimenti, il costrutto argomentale Det Nmc non può prescindere dall'esistenza di un luogo comune condiviso. ${ }^{18}$ Per questo motivo, quando una nominalizzazione non ricorre le ragioni sono da cercare sul piano semantico o pragmatico più che su quello sintattico:

(31) 'In effetti c'è stato un piccolo imprenditore edile che mi ha costretto ad ordinare in azienda una Jacuzzi color Ferrari Testarossa, mentre so di un professionista che ha demolito mezzo tetto perché voleva assolutamente montare nella mansarda una doccia Jacuzzi, che altrimenti non c'entrava". (LaR)

Dal momento che una Jacuzzi è, comunemente, una vasca da bagno, per alludere a una doccia della stessa marca non si potrà prescindere dalla menzione del nome comune.

Un luogo comune condiviso manca anche negli esempi seguenti, per cui difficilmente i marchionimi citati ricorreranno con lo stesso valore in funzione argomentale:

(32) Tende di pizzo alla finestra, grande stereo, un mazzo di tulipani e di iris sul tavolo basso. Lettone anonimo con salviette umidificate Pampers, quelle che si usano per i neonati, sul comodino. (LaR)

(33) questa fantasia gliela do a tre paia cinquemila \# vai \# la maggioranza son tutti rossi abbiamo solo una rimanenza $i$ collant e le calze setificate Santagostino (LIP FE 1)

Lo stesso si può affermare per i casi in cui il marchionimo modifica nomi concreti non riferiti a un prodotto:

(34) Sono stati 3 milioni gli uomini, le donne e i bambini che hanno varcato le soglie dei concessionari Fiat nella due giorni di "porte aperte" del 6 e 7 novembre. (CoLFIS, periodico)

(35) Nordio nelle risposte ricorda che i suoi bilanci da presidente, chiusi quasi tutti in nero, ripagano adeguatamente l'azionista. Nei suoi progetti c'è anche la costruzione di un grattacielo Alitalia a Manhattan. (CORIS MON2008_10)

17 Nell'elenco Migliorini inserisce anche ditta Hoepli, che però - lo si è visto - si caratterizza come predicazione denominativa.

18 Per i risvolti narratologici di questo concetto si veda quanto argomentato nei $\S 7.2$ e 7.3 . 
(36) Gavino Sanna, ideatore dello spot Parmacotto, mi ha raccontato che lei sul set a luci spente il prosciutto se lo mangiava davvero, seduta cordialmente alla stessa tavola della troupe. (CoLFIS, periodico)

(37) C'erano la bellezza di 1.800 invitati al megaparty Fiat per la presentazione della Punto, martedì 31 agosto nei giardini di Palazzo Reale a Torino. (CoLFIS, periodico)

(38) Invece, vecchio, orgoglioso, immarcescibile Cavallo Pazzo degli slalom, ti basta una brutta nottata da arrabbiato, per resuscitare [...], in fondo sei di parola, hai promesso il riscatto immediato proprio qui al Sestrière, la montagna Fiat che ti è amica e sponsor [...]. (CoLFIS, quotidiano)

Tra nomi comuni e nomi commerciali vi è un rapporto predicativo, come per scarpe Adidas (si pensi all'equivalenza con $N$ di Nmc), con la differenza che non si tratta di un rapporto tra produttore e prodotto e che quindi è meno plausibile l'equivalenza con l'uso argomentale Det Nmc. Usi argomentali, anzi, sarebbero qui potenzialmente ambigui: si pensi a "Gavino Sanna, ideatore del Parmacotto", di contro all'es. (36). In generale si osserva che la corrispondenza tra le strutture $N$ $N m c$ e Det Nmc è legata alla prototipicità del nome di prodotto rispetto al suo produttore; al fatto, insomma, che un determinato oggetto sia il prodotto per antonomasia di una marca.

(39) Ma con una particolarità: colletti e polsini a strisce bianche e blu che ricordano le tute Adidas. (CoLFIS, periodico)

In (39) l'uso argomentale del marchionimo - le Adidas con valore di 'le tute Adidas' - è bloccato, dal momento che le Adidas ricorre usualmente in riferimento a un paio di scarpe:

(40) Ho un figlio di quasi sedici anni. Non gli posso comprare le Adidas o lo zainetto Invicta. L'altra settimana mi ha portato al negozio qua sotto, voleva le scarpe Nike perché a scuola ce l'hanno tutti. (LaR)

Ci sono insomma nominalizzazioni più stabili di altre, che hanno acquisito un valore relativamente fisso in virtù del quale ricorrono con maggiore autonomia rispetto al nesso $N N m c$. Tale relativa fissità non si riflette peraltro in una registrazione nel dizionario, diversamente da quanto avviene per altri marchionimi divenuti luoghi comuni (cf. Parte I, 
$\S 3.3$ e 4.2): da un lato, per le caratteristiche morfosintattiche del costrutto (presenza del determinatore e suo valore); dall'altro, perché permane un aspetto metalinguistico, benché indiretto: le Adidas sono 'scarpe prodotte dall'azienda chiamata Adidas'.

La fissazione del luogo comune fa sì che, in certi casi, il nesso $N N m c$ sia percepito come ridondante rispetto a Det Nmc. L'esempio più eclatante è probabilmente quello di l'auto $N m c$, reputato troppo ridondante per poter ricorrere (Petit 2006: 692 mette l'asterisco a "Ma voiture Twingo est en panne"). A questo giudizio si contrappone una testimonianza autorevole:

(41) Umberto Eco gli ha risposto che "la televisione non c'entra assolutamente nulla. Che non fa niente di male contro i giornali. Si limita a dare le notizie in anticipo, come è naturale ed anzi fa pubblicità ai giornali con le sue rassegne stampa della notte. Sono i giornali italiani che devono smetterla di continuare a parlare di televisione e di esserle dipendenti. Non si è mai visto qualcuno fare tanta pubblicità al proprio concorrente. Come se le auto Fiat viaggiassero con la pubblicità della Citroen sulla fiancata". (LaR)

Non si tratta peraltro di un esempio isolato:

(42) Al gabbiotto della portineria si avvicinano con meno deferenza persino i ragazzotti del Pony Express, un anziano signore protesta perché c'è un'auto Fiat in seconda fila e lui è lì che aspetta da oltre un quarto d'ora. (LaR)

(43) E inoltre, più la locazione esotica d'Arte appare solennemente fotogenica, più l'orizzonte d'attesa nel fruitore di bellezza pubblicitaria si predispone automatico a passaggi di camion di birra Carlsberg, interventi di nuova moda Valentino e Armani, offerte speciali di auto Renault o lamette Gillette. (LaR)

(44) Attenzione però: come molte altre aziende italiane, la Fiat non vende solo un prodotto e le sue caratteristiche tecniche; insieme all'automobile Fiat, Lancia $o$ Alfa Romeo viaggia un patrimonio di stile e tradizione che soffrirebbe di un apparentamento troppo stretto con gli aspetti più "nerd" e da sala giochi americana della Rete. (CORIS MISCRiviste)

Su una scala di ridondanza, la struttura auto Nmc si situa certamente più in alto rispetto a pasta Barilla o borsa Vuitton; questo, però, non le impedisce di ricorrere più volte nei dati. ${ }^{19}$ Entrambi i costrutti, la Nmc

19 Nel corpus LaR si possono reperire poco meno di trenta marche diverse di automobili (da Alfa Romeo a Volvo, ma anche nomi di modelli, per es. Panda o 
e l'auto Nmc, sono potenzialmente disponibili ai parlanti; non si può postulare un'evoluzione da l'auto Fiat a la Fiat, che avrebbe soppiantato il primo costrutto per riduzione di ridondanza.

\subsubsection{Nessi N Nmc con nomi astratti}

La caratteristica dei nessi $N N m c$ con nome di prodotto messa in evidenza nelle pagine precedenti, ossia la coincidenza con il marchionimo in uso argomentale, non si applica alle strutture considerate in questo paragrafo, nelle quali il nome commerciale è una predicazione non nominalizzabile.

(1) Se l'obiettivo Fiat deve essere, come vanno ripetendo, la globalizzazione, questa non potrà che partire dal Sud America o comunque avere in quell'area uno dei suoi punti di forza. (LaR)

Obiettivo Fiat è un esempio di questa struttura. Un primo tratto combinatorio che viene alla luce è l'equivalenza con la struttura preposizionale obiettivo di(Det) Fiat (cf. §3.2.5):

(2) L'obiettivo di Fiat, superati i guai derivanti dalla stretta interdipendenza col Brasile - si tratta di due paesi vincolati al dollaro e nel caso dell'Argentina c'è anche la parità con il biglietto verde - è quello di tornare a quota 25-30 per cento in modo da poter poi controllare l'intero mercato sudamericano. (LaR)

(3) Se è vero che il primo grande obiettivo della Fiat è quello di entrare nel fiume impetuoso della globalizzazione per poi affrontare la scommessa delle alleanze con maggiore probabilità di successo di quanto non abbia avuto negli anni Ottanta, allora l'avvocato Fresco potrebbe essere l'identikit del presidente che Agnelli ha in testa. (LaR)

Tra nome comune e marchionimo si riconosce un rapporto qualificabile come possessivo: "Fiat ha un obiettivo".

Per la sua concisione la struttura $N N m c$, in cui resta implicito il tipo di rapporto tra nome comune e nome commerciale, si presta bene a ricorrere in porzioni testuali necessariamente ridotte quali i titoli:

Thema) che ricorrono una o più volte (Fiat, caso estremo, una trentina di volte) come modificatori del nome comune auto. 
"Assortimento Carrefour a prezzi Coop" è un sottotitolo reperito in un articolo di Spendere Meglio. ${ }^{20}$

Qual è la differenza tra moto Aprilia (\$2.2.1, es. 17) e Dominio Aprilia, nell'esempio seguente?

(4) Dominio Aprilia, nel Gp di Spagna: en plein nelle 125, con i primi tre posti, e prima nelle 250, con il francese Ruggia. (CoLFIS, quotidiano)

Per entrambi i costrutti si riconosce la coincidenza con la struttura $N$ di Nmc; invece l'equivalenza con l'uso argomentale del marchionimo (l'Aprilia, un'Aprilia) si dà solo per moto Aprilia. Aprilia non è "un dominio" come è "una moto"; in (4) non è possibile prescindere dal nome comune.

In altre parole, e tenuto conto della possibilità di alternare, come modificatori, marchionimo semplice e complemento di specificazione, in questo tipo di costrutti il nome commerciale è funzionalmente equiparabile a un aggettivo di relazione: ${ }^{21}$ è questione di un dominio relativo ad Aprilia - nella fattispecie, delle moto omonime - così come, nei brani seguenti, si parla di "vicende" o "casi" relativi a imprese:

(5) Ieri mattina, a Piazza degli Affari, la vicenda Fiat è stata al centro di tutti i commenti. (LaR)

(6) Il nuovo scandalo alla scommesse [sic], i fondi neri, la crisi del Col, la vicenda Adidas, dimostrano che le mie idee sono giuste: lo sport è diventato troppo complesso perfino per chi ha tra le mani una buona professionalità. (LaR)

(7) Per scoprirlo, basta sfogliare il dossier d'inchiesta della Cob, il gendarme della Borsa francese, sul caso Perrier: 51 riservatissime pagine, di cui il 4 febbraio è stata resa pubblica solo la conclusione. (CoLFIS, periodico)

In questi esempi si osserva inoltre che il nome commerciale ha funzione soggettiva rispetto ai nessi in cui ricorre: un equivalente di (4) sarà "Aprilia ha dominato" e, per (5-6), "Fiat/Adidas è coinvolta in una vicenda".

20 Nel prosieguo si legge: "pur avendo ripreso le filiali svizzere del discounter francese Carrefour, Coop non ha evidentemente ripreso i suoi prezzi" (SM 2/09).

21 Lo afferma Migliorini (1952: 118) in merito ai costrutti del tipo piazza Garibaldi, nei quali "il cognome ha già pressappoco il valore di un aggettivo di relazione". 
L'interpretazione del nesso $N$ Nmc, come nota Jonasson (1994: 107-108), è fortemente soggetta a conoscenze di tipo pragmatico o enciclopedico, in particolare quando vi ricorrono nomi astratti, come in questi casi: la struttura lascia implicito il rapporto che vige tra i due nomi; ${ }^{22}$ pertanto si rivela necessario un sapere enciclopedico non solo per risalire al referente del nome proprio ma anche per stabilire il rapporto che esso instaura con il nome comune. Questo avviene perché, scrive Kleiber (1985: 6), nei nessi di questo tipo - anche con nomi propri prototipici, dei quali si occupa lo studioso francese - il nome comune non denota generalmente una classe di referenti nominabili: di fronte a costrutti come l'année Victor Hugo ci si interroga sulla relazione tra i due nomi e sulle ragioni (di ordine enciclopedico, appunto) per le quali un tale nome comune è ribattezzato così. Non di rado, dunque, come nell'es. (4), il brano fornisce informazioni alla luce delle quali è possibile interpretare il nesso (dominio Aprilia = le motociclette di marca Aprilia hanno occupato diverse posizioni sul podio nelle gare del Gran Premio). Se queste informazioni mancano, come nel brano seguente, il costrutto rimane opaco:

(8) Così [Fausto Bertinotti] ha preso le distanze dal Pds affermando che l'accordo Fiat "è un bidone". (CoLFIS, periodico)

L'estratto non permette di capire se l'accordo Fiat sia un accordo che coinvolge Fiat, un accordo tra diversi gruppi d'interesse all'interno dell'impresa o un accordo "in stile Fiat", che riguarda solo indirettamente l'azienda.

Ecco altri esempi di relazione soggettiva tra marchionimo e nome modificato:

(9) La crisi Fiat scoppia all'Alfa Romeo di Arese. (CoLFIS, quotidiano)

(10) Che cosa vuol dire questo andamento tradotto in cifre sull'intero anno? Innanzi tutto un fatturato di gruppo sui 37 mila miliardi e poi un risultato operativo di 2.700 miliardi, investimenti per 3 mila miliardi, autofinanziamento oltre i 4 mila miliardi, indebitamento sui 1.900 miliardi che equivale a un'ulteriore riduzione sull'andamento registrato a fine aprile. Fin qui la prudenza Fiat. (LaR)

22 Ciò vale in generale per tutte le combinazioni $N$ Npr: il loro significato non è codificato, per cui esse necessitano di un calcolo interpretativo (Kleiber 1985: 4). 
(11) La Jugoslavia aveva un suo cavallo di battaglia in un modello semplificato della vecchia Cinquecento, inizialmente su licenza Fiat, prodotta nella fabbrica di Kragujevac. (CoLFIS, libro - D. Volcic, Sarajevo. Quando la storia uccide)

Il valore di soggetto del marchionimo si riconosce nelle possibili parafrasi "Fiat è in crisi" (9), "Fiat è prudente" (10), "Fiat concede una licenza" (11).

Il tipo soggettivo si declina anche in termini di relazione di possesso $(N N m c=N m c$ ha un $N)$, come visto per obiettivo $N m c$ :

(12) E la fissazione è davvero dei giornalisti, e non piuttosto dei vertici Pirelli? (CoLFIS, quotidiano)

(13) Quanto più aumenta la mole di notizie in mano ai giudici, tanto più la posizione del gruppo torinese sembra governata non da un patto (né tantomeno da un "patto scellerato" come lo ha chiamato il manifesto) ma dallo sfondamento quasi completo della linea difensiva Fiat da parte dei giudici di Mani Pulite. (CoLFIS, quotidiano)

(14) I Francesi continuano a studiare il piano, soprattutto perché temono che un insuccesso delle trattative apra la strada ad un ingresso della Toyota nel capitale Fiat. (CoLFIS, quotidiano)

Contengono nomi astratti anche i nessi degli esempi che seguono:

(15) Schweitzer non sarebbe molto lontano dalle posizioni di Jacques Calvet, presidente del gruppo Peugeot-Citroen, che ha liquidato con una battuta l'ipotesi di un'intesa Fiat-Renault: "Non auguro nemmeno al mio peggior nemico un simile progetto". (CoLFIS, quotidiano)

(16) Ha promosso l'accordo Pirelli-Dunlop, ed è stato un fallimento. (CoLFIS, libro - Enzo Biagi, I come Italiani)

Tanto per intesa quanto per accordo, seguiti da due marchionimi coordinati asindeticamente, si può prevedere una coincidenza con un nesso preposizionale introdotto da tra: "un'intesa tra Fiat e Renault", "l'accordo tra Pirelli e Dunlop". 


\subsubsection{Nessi N Nmc con nomi [+ umani]}

Gli esseri umani, scrive Kleiber (1985: 6), sono referenti nominabili per eccellenza: "Notre savoir extra-linguistique fait que nous leur associons en premier lieu l'idée de porteur d'un nom”. Per questo motivo, continua lo studioso, di fronte a nessi che combinano un nome comune (relativo a una professione, un grado di parentela ecc.) e un nome proprio, si tenderà a interpretarli come casi di "denominazione totale": $N$ $N p r$ è 'il $N$ chiamato $N p r$ '.

Uno sguardo ai costrutti con nomi commerciali mostra che l'ipotesi di Kleiber tiene se con "nomi propri" si intendono i nomi prototipicamente assegnati a entità umane, ossia gli antroponimi. La combinazione di un nome caratterizzato dal tratto [+ umano] e di un marchionimo non porta alla stessa interpretazione denominativa.

(1) Sul pilota Schumacher Montezemolo poi aggiunge: "È uno che lavora costantemente con la squadra, che fa un grande lavoro che aiuta tutti a gestire meglio le cose. [...]" (LaR)

(2) "Sono felice per i miei tifosi che mi hanno seguito qui in Belgio", prosegue Schumacher, pilota Mercedes in prestito alla Benetton [...]. (CoLFIS, quotidiano)

In termini di equivalenza, la differenza tra (1) e (2) si può esprimere come segue: a "pilota Schumacher" corrisponde "Schumacher è un pilota", mentre a "pilota Mercedes" non corrisponde "Mercedes è un pilota" ma, semmai, "Mercedes ha un pilota".

Come (2) funzionano i costrutti seguenti, con nomi riferiti a professioni e ruoli svolti in seno a un'azienda:

(3) Ruggero Gorgoglione, 25 anni, operaio Fiat e Maria Rosaria Triola, 24 anni, casalinga, via Lulli 3, entrambi incensurati, stavano circondando di attenzioni la loro bambina di 2 anni, che portavano a fare una passeggiata. (CoLFIS, quotidiano)

(4) "Attraverso di lei colpiscono l'anarchismo di Carrara", insiste lo zio, Alfonso Nicolazzi, ex steward Alitalia, ora tipografo. (CoLFIS, quotidiano) 
(5) Dirigenti Microsoft hanno confermato che la società, pur avendo iniziato a sviluppare una propria tecnologia whiteboard, sta anche valutando la possibilità di acquisire un prodotto sviluppato da terzi. (CoLFIS, periodico)

(6) Un esito forse non imprevedibile quando a parlare sono iscritti imprenditori "duri" come Marco Tronchetti Provera, vicepresidente esecutivo e amministratore delegato Pirelli $[\ldots . .$.$] . (CoLFIS, quotidiano)$

(7) Migros è scettica riguardo ai confronti prezzi. «Ci sono troppe variabili» dichiara Francesca Sala portavoce Migros Ticino. (SM 4/05)

(8) La volata è imponente. Non ci sono poliziotti Kodak, in giro. La zona del traguardo è sgombra. (CoLFIS, quotidiano $)^{23}$

Qui si riconosce un'equivalenza con $N d i N m c$, per la quale si rimanda al §3.2.3, infra. Essa è valida anche per i brani a seguire, con nomi umani non riferiti a professioni:

(9) In realtà l'inglese è il protetto McLaren e Alliot è il pupillo Peugeot. Vedremo chi sarà scelto, nel caso di un "no" di Prost. (CoLFIS, periodico)

(10) E chi è stato più colpito dai messaggi pubblicitari della Casa? I clienti Volkswagen, naturalmente. (CoLFIS, periodico)

(11) Martin Sutnar di Migros-Marketing [...] aggiunge: «Se la clientela Migros dovesse pagare la crema Sun Look ancora meno, lo declasserebbe a prodotto a buon mercato». (SM 2/05)

(12) Mentre si passa il tempo chiacchierando un po' con tutti, nel classico clima amichevole che per qualche strano motivo sembra sempre svilupparsi tra gli utenti Apple in raduno, arriva una ragazza a distribuire i volantini dell'offerta della 3: chiamali scemi. (CORIS MON2008_10)

Tra i due elementi del nesso si riconoscono perlopiù rapporti di tipo possessivo e/o soggettivo: "Peugeot ha un pupillo", "Migros ha una clientela".

Un nome [+ umano] che ricorre modificato da marchionimo è lo stesso uomo:

23 Si tratta di un uso traslato del nome commerciale, riferito al fotofinish nelle gare di ciclismo. 
(13) I nazionali francesi sono furenti con la Adidas, che vieta loro la libertà nella scelta degli scarpini da gioco. Mercoledì sera, le tre bande bianche erano visibili solo sulle scarpe di Desailly, Barthez e Zidane, già uomini Adidas. Gli altri le avevano "oscurate", coprendole. (CORIS STAMPAQuotidiani)

(14) Se ne deduce che il governo, di cui Ferrara è portavoce, non ha nessuna obiezione di principio a che 'uomini-Fininvest' passino a dirigere la Rai: prospettiva che, invece, non piace alle forze di opposizione né alla Lega. (LaR)

(15) Per la gente di Cory Enrile è stato fino a febbraio di quest'anno tanto nemico e tanto temuto quanto Marcos. Enrile è stato per vent'anni il braccio destro della dittatura, uno degli uomini Rolex, uno degli architetti della legge marziale cui Marcos in ricordo aveva regalato un Rolex d'oro. (LaR)

(16) Roberto Testore è un signore di 43 anni, ingegnere meccanico, formato alla scuola del Politecnico di Torino, sposato e padre d'un figlio, alle spalle una carriera tutta di marca Fiat, con una sola, breve parentesi d'un paio d'anni in una società dell'Ifi vicina all'Unicem di cui il padre era stato per un paio di decenni amministratore delegato. Insomma un uomo Fiat a tutto tondo [...]. (LaR)

(17) Gli uomini-Fiat c'erano tutti e, accanto a loro, sedevano gli altri esponenti della grande impresa locale, quasi a voler testimoniare con la loro presenza il sostegno morale, se non proprio elettorale, ad un Pri passato all'opposizione. (CoLFIS, quotidiano)

Il contesto permette di interpretare il nesso uomo $\mathrm{Nmc}$ di volta in volta in maniera pertinente: in (13) c'è un riferimento alla sponsorizzazione da parte di Adidas, in (14) e (17) si allude ai manager della ditta (cf. infra, $\$ 3.2 .3$, ess. 16-17).

L'accostamento di nome [+ umano] e nome commerciale, insomma, non ha carattere di predicazione denominativa, come nel caso degli antroponimi; si tratta ancora una volta di una struttura che lascia implicito il tipo di relazione tra i due nomi. Il marchionimo, apposto come etichetta a nomi riferiti a esseri umani, giunge persino a evocare un'identificazione totale con l'azienda (18) o il prodotto (19):

$$
\text { Come Vincere [...] L'orgoglio di essere Persone Barilla (<www.barillagroup.it }>24 \text { ) }
$$


(19) Forum - Un luogo tutto per te in cui condividere racconti ed esperienze con le altre mamme Pampers! (<www.pampers.it $>25$ )

\section{Conclusioni}

La struttura $N N m c$, di cui questo capitolo ha colto alcune sfaccettature sintattiche, sfugge ad analisi del marchionimo improntate a una prospettiva onomastica, dal momento che il nome commerciale vi compare in funzione predicativa e non argomentale. ${ }^{26}$ Con essa si ripropongono questioni insolute della ricerca sui nessi che contengono nomi propri prototipici.

In queste pagine si è operata una prima distinzione sulla base dell'eventuale carattere denominativo del modificatore rispetto al nome comune: il marchionimo costituisce una modificazione di tipo denominativo di quanto precede oppure no?

Modificazioni denominative si riconoscono nei nessi "lo yogurt Alleluya", "la ditta Codemi", "la marca Invicta", che si contraddistinguono per la possibilità, in generale, di nominalizzare l'attributo (non così spesso con marca), mentre non vi è corrispondenza con il costrutto $N$ di Nmc. In questa prima tipologia di struttura il nome comune ha carattere di classificatore ${ }^{27}$ e può pertanto essere inteso come una sorta di apposizione interna (cf. "Nmc è un'azienda/una marca..."); d'altro canto, è pure possibile intendere il nome commerciale come un modificatore di tipo nominale (cf. La Fauci 2009: 158).

Altri costrutti presentano invece una predicazione non denominativa, accomunabile a quella manifestata da aggettivi relazionali: "gli

$25<$ www.pampers.it/forum> (15.7.2015).

26 Si sarà forse osservata la scarsa presenza di determinati nomi commerciali in funzione predicativa: nomi (di prodotto) quali Kleenex, Barbie, Nutella. Non mancano del tutto usi predicativi di tali nomi ma, quando ricorrono, presentano perlopiù valore tendente al metaforico (cf. §6.2).

27 Cf. quanto afferma Van Langendonck (2008: 816) a proposito del "basic level meaning" del nome proprio, che sarebbe appunto indicato dal nome comune nelle varie strutture che combinano nomi propri e comuni. 
orologi Swatch", "la vicenda Adidas", "la prudenza Fiat", "dirigenti Microsoft". Il nome comune rappresenta qui il nucleo del nesso. ${ }^{28}$

In questi casi è solitamente possibile stabilire un'equivalenza con la struttura $N c \operatorname{di}($ Det $) ~ N m c$. Vi è inoltre la possibilità - in ragione di conoscenze condivise, di un alto grado di ridondanza - di un uso argomentale del solo marchionimo (Det Nmc), combinato a un determinatore che non costituisce variante libera. Da modificatore o attributo, il marchionimo diventa esso stesso argomento e ne assume la funzione quando il nome modificato è così noto che si sa a cosa sia riferito ("per antonomasia"). Succede spesso nella lingua: "l'uomo è al primo piano" potrà sentirsi dire chi, in un negozio d'abbigliamento, sta cercando il reparto dedicato agli abiti da uomo.

L'analisi funzionale permette di distinguere costrutti in apparenza uguali, come azienda $N m c$, che presenta due diversi valori combinatorî del nome commerciale. Per disambiguarli si può allora chiedersi: c'è equivalenza con l'azienda di $\mathrm{Nmc}$ ?

In relazione agli usi predicativi del nome commerciale si pone con particolare insistenza la questione dei marchionimi come aggettivi (funzionali). Noailly (1990: 104) afferma che il costrutto $\mathrm{N} \mathrm{Npr}$ con relazione di "complementazione" ("determinazione" per Jonasson 1994: 105; qui si è parlato di aggettivi relazionali) ricorre con maggior frequenza con quei nomi propri che non dispongono in maniera sistematica di un aggettivo: antroponimi, nomi di società e di organismi, di contro a nomi di città e nazioni. In effetti i nomi commerciali presentano solo occasionalmente derivati di forma aggettivale del tipo barilliano (cf. infra, cap. 5). Gary-Prieur (1994: 90), dopo aver ripreso le osservazioni di Noailly, aggiunge: "Ne peut-on en conclure que le

28 Dal punto di vista interlinguistico, in particolare in relazione al tedesco, si ipotizza che il primo gruppo (con predicazione nominale) sia accomunabile ai nessi che contengono "Deskriptoren", secondo la terminologia di Kolde (1995: 406): il tipo Herr Miller, Bäcker Otto Miller; mentre il secondo gruppo ricorre perlopiù sotto forma di nomi composti: der Eiffelturm, die Goethestrasse. Mentre il tedesco presenta due strutture anche formalmente diverse (Herr Müller/Bahnhofstrasse), in italiano i due tipi sono superficialmente simili (signor Rossi/via Roma), ciò che complica ulteriormente la questione del rapporto sintattico tra i due nomi. Sulle differenze tra lingue germaniche e lingue romanze a questo proposito cf. Jonasson (1995: 92). 
nom de personne en l'absence d'adjectif dérivé, fait lui-même office d'adjectif, c'est-à-dire qu'il est apte à attribuer des propriétés de son référent au nom qui le précède?". La risposta è: sì, e per questioni sintattiche, combinatorie, prima ancora che referenziali. I marchionimi ricorrono come predicazioni - non solo, si noti, nei costrutti N Nmc-; come aggettivi invariabili, che possono modificare direttamente nomi comuni, anche nei casi in cui gli antroponimi ricorrerebbero introdotti da una preposizione: di contro a "l'obiettivo Fiat" e "la risposta Fiat" si avranno per es. "l'obiettivo di Ugo" e "la risposta $d i$ Eva".

Alla luce di queste osservazioni è lecito affermare che il carattere di aggettivo è, nei nomi commerciali, più forte ancora che nei nomi di persona. Non si oserà pertanto sostenere che i nomi commerciali sono nomi propri prototipici, anche se, secondo il punto di vista qui illustrato, si potrebbe forse essere tentati di farlo. 


\section{Nessi preposizionali}

\section{Introduzione}

I nessi in cui il nome commerciale è introdotto da una preposizione (Prep Nmc), superficialmente simili tra loro, svolgono funzioni assai diverse entro la proposizione, in dipendenza dalle relazioni sintattiche che instaurano con le altre parti di essa (cf. La Fauci 2009: 80). Può trattarsi da un lato di nessi argomentali (per es. soggetti nozionali di costrutti passivi); dall'altro di modificazioni predicative, distinguibili a un primo livello in complementi del nome (nessi nominali introdotti da una preposizione, perlopiù di; cf. La Fauci 2009: 159) e complementi del verbo, che "stanno fuori della portata di legittimazione dei predicati" e sono quindi definibili come supplementi predicativi (La Fauci 2009: 195). In ossequio a queste differenze funzionali, l'analisi dei nessi preposizionali si articola in quattro parti.

Nel §3.1 sono studiati i nessi legittimati dal predicato, che sono manifestazioni di funzioni argomentali. Vi si annoverano innanzitutto (§3.1.1) nessi con funzione argomentale nucleare (cf. La Fauci 2009: 80): soggetti nozionali di costrutti passivi (la mostra è prodotta da Swatch) e oggetti indiretti (giovare alla Fiat). Si riconoscono poi (\$3.1.2) altri argomenti legittimati dal predicato e retti da esso mediante una preposizione, ovvero i complementi preposizionali del predicato (cf. La Fauci 2009: 81-82): da vivere di Kodak ai costrutti, caratteristici dei nomi commerciali, investire in Nike o entrare alla Honda.

Nel $\$ 3.2$ si analizzano i complementi di specificazione $(N d i$ $N m c$ ), ossia i nessi preposizionali introdotti da $d i$ che fungono da modificazione predicativa di un nome o nesso nominale (cf. La Fauci 2009: 159-160). Tra di essi si riconoscono vari tipi di rapporti. Vi sono innanzitutto i complementi di specificazione a valore soggettivo e oggettivo (\$3.2.1; cf. La Fauci 2009: 160-161), ossia i costrutti $N$ di $N m c$ in cui la natura predicativa del nome modificato - per es. prodotto, vendita 
- è meglio evidente da un punto di vista sperimentale. In questi casi il rapporto tra nome modificato e marchionimo equivale al rapporto tra un predicato verbale e il suo argomento (l'acquisto di Ferrarelle: 'qualcuno acquista Ferrarelle' oppure, a seconda dei casi, 'Ferrarelle acquista'). Numericamente importanti sono pure le ricorrenze della relazione tra opera (prodotto) e artefice (produttore): $i$ cosmetici della Revlon (\$3.2.2). Si studiano poi i nessi che modificano nomi umani, come il presidente della Coop (\$3.2.3); le correlazioni di contenente e contenuto: una lattina di Coca-Cola (\$3.2.4); i marchionimi con funzione di aggettivi relazionali, per es. l'obiettivo della Fiat (\$3.2.5). Nel trattare i costrutti con $d i$ si riflette in particolare alla questione dell'equivalenza con nessi comparabili in cui la preposizione non ricorre $(N$ $\mathrm{Nmc}$ ), in contesti che occorrerà specificare. In entrambi i casi, tanto di Nmc quanto il semplice $N m c$ si comportano funzionalmente come predicazioni.

Il §3.3 si concentra sui complementi nominali introdotti dalla preposizione da (sorriso da Colgate).

Infine, nel §3.4 sono analizzati alcuni esempi di supplementi predicativi (o "avverbiali di frase"), vale a dire nessi preposizionali - con i marchionimi, tipicamente, a interpretazione locativa - che si situano al di là della proposizione semplice (cf. La Fauci 2009: 172; 195-211). Per i supplementi predicativi a valore locativo (\$3.4.1), quali per es. sono operaio alla Michelin o in vendita alla Migros, si studierà l'equivalenza funzionale tra strutture formalmente diverse, che presentano variazioni di preposizione (in vendita presso/da/alla Nmc; lavorare in/ alla $\mathrm{Nmc}$ ). Si considera inoltre la questione dell'articolo e, correlativamente, della proprialità, in particolar modo nell'alternanza tra $a(D e t)$ $\mathrm{Nmc}$, dove ricorre l'articolo, e da Nmc, senza determinatore. Si considerano poi altri supplementi predicativi, introdotti perlopiù da in, con interpretazione modale o strumentale: in Mercedes (mezzi di trasporto; §3.4.2), in Lacoste (capi d'abbigliamento; §3.4.3). 


\subsection{Funzione argomentale}

\subsubsection{Nessi preposizionali con funzione argomentale nucleare}

Le preposizioni $a$ e $d a$ introducono nomi commerciali che manifestano, rispettivamente, funzione di oggetto indiretto e di soggetto nozionale in costrutti passivi. In entrambi i casi tali nessi preposizionali sono legittimati dal predicato.

(1) Resta però incandescente la questione Malpensa: se per il ministro Bersani (in un'intervista a L'espresso) «non si può chiedere ad Alitalia di salvare Malpensa», il patron di Air One Carlo Toto [...] rilancia: «La partita Alitalia non è chiusa $[\ldots] »$. (CORIS MON2008_10)

(2) $[\ldots]$ il fatto di non avere forti concorrenti esteri che operano direttamente in Italia può oggi solo giovare alla Fiat nella sua politica di espansione a Est. (CoLFIS, periodico)

(3) Quando si dice bevande gassate, si pensa subito alla Coca-Cola o alla Pepsi. Quando si dice automobile, si pensa alla General Motors. (CORIS STAMPA Supplementi)

In questi costrutti il marchionimo introdotto dalla preposizione $a$ (con o senza articolo) ha funzione di oggetto indiretto.

Nei seguenti casi il nesso preposizionale con $d a$ ha valore funzionale di soggetto nozionale (il cosiddetto complemento d'agente; $\mathrm{cf}$. La Fauci 2009: 95):

(4) Wilco Zeelenberg è stato scelto da Yamaha come team manager per dirigere la squadra di Jorge Lorenzo in MotoGP. (CORIS MON2008_10)

(5) Young, oggi sessantacinquenne e impegnato per la difesa dei diritti dei lavoratori, è stato assunto dalla Nike. (CORIS STAMPASupplementi)

(6) Poco tempo dopo altri due incidenti. Un vigile urbano (di scorta al presidente del Consiglio Goria) cadde dalla Guzzi e un agente della polizia stradale rimase gravemente ferito. L'inchiesta del magistrato è stata accolta con stupore dalla Moto Guzzi. (LaR) 
Si hanno inoltre costrutti passivi ridotti, che fungono da modificatori di tipo predicativo rispetto a un nesso nominale:

(7) Prosegue fino al 10 ottobre Happy School, la manifestazione organizzata da Coin per soddisfare le esigenze degli studenti. (CoLFIS, periodico)

(8) Dopo l'adesione all'iniziativa Eridania da parte dell'Anb, dell'Abf e dall'Abv (cioè dell' $80 \%$ circa dei bieticoltori organizzati) il traguardo dei 5.300 .000 quintali di zucchero prodotti da Eridania, obbiettivo minimo per il varo dell'operazione "azione verdi", pareva scontato. (LaR)

(9) I carabinieri hanno acquisito presso il ministero del Tesoro, per ordine del pubblico ministero Maria Cordova, la documentazione riguardante le gare indette a suo tempo dal dicastero e dal ministero della Difesa per la forniture di sistemi di videoscrittura prodotti dalla Olivetti. (LaR)

Negli esempi ricorrono marchionimi determinati e non-determinati, tanto con gli oggetti indiretti quanto con i soggetti nozionali di costrutti passivi (cf. §1.1.2). Per il tratto [+ umano] del soggetto legittimato dal predicato, visibile per es. in (6) - "L'inchiesta del magistrato è stata accolta con stupore dalla Moto Guzzi" -, si rimanda al $\S 4.1$.

\subsubsection{Complementi preposizionali del predicato}

Tra i nessi argomentali legittimati dal predicato e retti da esso mediante una preposizione (cf. La Fauci 2009: 81) non si annoverano soltanto soggetti logici e oggetti indiretti ma anche altri argomenti:

(1) A Rochester, una gagliarda cittadina dello Stato di New York, 34 mila persone su 232 mila vivono di Kodak. (STAMPASupplementi)

(2) Intanto nella colonia-Italia si continua sulla strada delle privatizzazioni. Ora tocca all'Alitalia e il pericolo è che i cittadini paghino le spese salvando gli interessi usurai delle banche. (CORIS MON2008_10)

(3) Antesignano della comunicazione, [Mario Pavesi] aveva affidato il messaggio ai creativi dell'epoca, ribattezzando in seguito i suoi "Biscottini" in "Pavesini". (CORIS STAMPAQuotidiani) 
A legittimare il nome commerciale sono predicati complessi formati da verbo e preposizione: vivere di, toccare a, ribattezzare (qualcosa) in. Tipicamente sfilare per è seguito dal nome di una griffe: ${ }^{29}$

(4) Le giovani modelle che ieri hanno sfilato per Valentino si assomigliano come tanti asparagi. (LaR)

(5) Ha dato forfait invece all'ultimissimo momento ('Mi sento male, non lo faccio') Valeria Marini, che doveva sfilare per Missoni. (LaR)

(6) Gli fa eco una grande indossatrice del passato, Elsa Martinelli, che debuttò nel '54 nella Sala Bianca di Palazzo Pitti, sfilando per Emilio Pucci: "Forse la gente non ha idea di quanto faticoso sia questo lavoro $[\ldots]$ ”. (LaR)

Un altro predicato complesso che ricorre di frequente con marchionimi è investire in:

(7) Per regolamento - dice Gregory Knott di Momentum, fondo offshore amministrato dalla Banca delle Bermuda in Lussemburgo, che investe anche in Nike, Adidas e Callaway Golf - non possiamo incoraggiare investitori che non si rendono conto dei rischi. (CORIS STAMPASupplementi)

(8) E su questo punto in quegli anni alla Fiat si confrontarono due diverse impostazioni: era più conveniente investire in Marelli o era meglio concentrare le risorse sull'auto e cominciare a rivolgersi, per gli acquisti di componenti ad alto contenuto tecnologico, a Bosch o Valeo? (CORIS STAMPASupplementi)

(9) Stiano dunque tranquilli e fiduciosi i 72 mila azionisti della Fiat. La società, dice Agnelli, "ha una struttura equilibrata e flessibile, capace di affrontare la concorrenza, di cogliere i voleri del mercato e di remunerare adeguatamente il proprio capitale". In altre parole quanti hanno investito in Fiat non avranno certo a pentirsene. $(\mathrm{LaR})$

(10) "Il fatto che un imprenditore come Raul Gardini, proveniente dal mondo agricolo - ha detto a Mantova Luigi Lucchini - decida di investire in Montedison, non può che essere interpretato in modo positivo. [...]". (LaR)

I dizionari riportano, alla voce investire, il complemento preposizionale introdotto da in, con il significato di "impiegare un capitale in

29 E pure fare la modella per: "Cosa faceva prima di diventare caporedattore per la finanza? «La modella per Adidas, Puma, Nike. [...]»»"(CORIS STAMPAQuotidiani). 
qualcosa".$^{30}$ Investire in $\mathrm{Nmc}$ è la forma più diffusa, ma non mancano casi in cui il marchionimo è articolato: ${ }^{31}$

(11) La Chrysler intende ritirarsi dalla Diamond star motors, la joint venture con la Mitsubishi. Secondo quanto riporta oggi il quotidiano Asahi Shimbun, il gruppo Usa ha informato il partner di non essere più in grado di investire nella Diamond, di cui ora la Chrysler controlla il 50 per cento [...]. (LaR)

(12) Era stato lo stesso Iacocca a invitare il finanziere californiano [Kirk Kerkorian] a investire nella Chrysler nel 1992. (LaR)

(13) Pur non felici per quel che accade a Wall Street, alla Fiat tendono a sdrammatizzare. La nostra - dicono - era concepita come una iniziativa di medio-lungo termine $[\ldots]$. E poi, convincere gli americani a investire nella Fiat non è né facile né rapido. $(\mathrm{LaR})$

La presenza della determinazione non comporta una variazione di significato.

Un altro predicato accompagnato da complemento preposizionale è entrare in $N m c:^{32}$

(14) Paolo Giacca, 48 anni, è entrato in Ferrarelle in qualità di direttore vendite. Ha maturato esperienze in Lancaster, Vini Santa Margherita, T\&C, Carlsberg e Johnson Wax. (CORIS MON2005_07)

(15) Ferrero, che è nato a Rivoli (Torino) il 16 aprile 1929, è entrato in Fiat nel 1956 dove ha ricoperto numerosi incarichi, tra i quali quello di direttore generale della Piaggio di Genova, e poi di vicedirettore generale della Fiat auto spa. (LaR)

(16) Se così fosse i libici incasserebbero una somma che risulta di gran lunga superiore a quella sborsata complessivamente nel ' 77 per entrare in Fiat e cederebbero però soltanto una parte delle azioni in loro possesso. (LaR)

30 Cf. Treccani s.v. investire 2 : "Impiegare utilmente risparmî (o somme in genere) in capitale o in imprese fruttifere, fare degli investimenti: $i$. grosse somme in case, in terreni, in azioni; $i$. il ricavato di una vendita in titoli di stato" $(<\mathrm{www}$. treccani.it/vocabolario/investire/>, 4.6.2015).

31 Ricorre inoltre l'espressione nominalizzata: "L'operazione [...] comporta globalmente per Ifi-Ifil un investimento in Fiat di circa 900 miliardi” (CoLFIS, periodico).

32 Con l'equivalente nominalizzato: "Il mio ingresso in Mondadori è un investimento" (LaR). 
Un gruppo di nuovi azionisti di tutto rilievo si prepara ad entrare in Mondadori, grazie all'aumento di capitale e al ritorno in Borsa. (LaR)

La particolare interpretazione di questa struttura, correlata a un uso figurativo di entrare (cf. Treccani s.v. entrare ${ }^{2 a}$ ), pare caratteristica dei nomi commerciali. Due le letture principali: quando soggetto di entrare in sono individui (14-15) il costrutto equivale a 'essere assunto', 'entrare alle dipendenze di $N m c^{\prime},{ }^{33}$ quando il soggetto è un'entità collettiva (1617: "Un gruppo di nuovi azionisti", "i libici") si propenderà invece per 'acquistare titoli di $N m c$ ' o 'investire in $N m c$ '. ${ }^{34}$

Una struttura simile a quest'ultima ricorre inoltre con marchionimo determinato:

(18) La famiglia Rocca, titolare della Techint e di altre importanti attività in Argentina, è molto interessata ad entrare nella Pirelli e a questo fine, secondo alcuni già da tempo, va acquistando azioni Pirelli \& C. e Caboto sul mercato. (LaR)

(19) C'è chi dice che la Volkswagen è vicina a entrare nella Seat e chi assicura che la Chrysler, oltre a costruire una vettura assieme alla Mitsubishi, sia interessata all'Alfa Romeo. (LaR)

(20) Lavoratori e sindacati sono pronti a ricusare l'accordo che ha portato la Fiat ad entrare nella Maserati e ad aprire le porte ad una casa automobilistica straniera. (LaR)

L'unica interpretazione possibile è quella di tipo finanziario (acquisto di azioni), non certo quella riferita all'impiego presso una ditta, bloccata peraltro dal tipo di soggetto del predicato entrare. Nei dati si individua quindi una tendenziale differenza tra entrare in $N m c$, che può valere come indicazione tanto di un investimento quanto dell'inizio di un rapporto di lavoro, e entrare nella $\mathrm{Nmc}$, riferito alla partecipazione

33 Con questo significato ricorre inoltre la struttura arrivare in: "Presidente della Fiat Auto, proprio in seguito all'abbandono di Umberto, Garuzzo era arrivato in Fiat grazie a Carlo De Benedetti" (LaR).

34 Ovviamente non sono esclusi altri valori. In una pubblicità di Banca Mediolanum reperita su LaRepubblica (novembre 2016) si legge per es.: "Scegli la sicurezza, entra in Mediolanum." Ancora una volta, l'intento comunicativo potrebbe fare la differenza; bisognerebbe verificare la ricorrenza di questa struttura e del relativo valore in testi non pubblicitari. 
finanziaria alle sorti dell'impresa, mentre l'uso con il primo valore non ricorre nei dati analizzati.

Vi è poi la struttura entrare alla Nmc:

(21) Mio padre è operaio, lavora in casa per l'industria delle armi, che in Val Trompia è una fonte di lavoro sicuro. Anch'io pensavo di entrare alla Beretta, poi ho creduto alle mie possibilità nel calcio. (LaR)

(22) Questi operai, dicono quelli della Honda, sono il nostro fiore all'occhiello: li abbiamo selezionati non solo seguendo il criterio dell'abilità e della fedeltà all'azienda, ma anche in base al loro curriculum: abbiamo dato la precedenza a tutti quelli che, prima di entrare alla Honda, avevano svolto una lunga attività artigianale... (LaR)

(23) Prima di entrare alla Thomson, nel febbraio 1983, il presidente designato è stato membro del consiglio d'amministrazione e condirettore aggiunto della Chargeurs. (LaR)

(24) Per entrare alla Lepetit, invece, è spesso necessario un curriculum più sostanzioso. (LaR)

(25) Guidotti nacque a Bellagio (sul Lago di Como) ed entrò da giovanissimo all' $A l f a$, dove gli venne assegnato a soli 26 anni il duplice ruolo di collaudatore e di pilota ufficiale [...]. (CoLFIS, quotidiano)

In questi casi il costrutto è interpretabile nei termini di una relazione di lavoro ('essere assunto da'). Vi è dunque corrispondenza con la struttura entrare in Nmc, con una differenza: la determinazione. Mentre con entrare a l'articolo (determinativo, singolare e di norma femminile) è sistematicamente presente, nel caso di entrare in esso non ricorre, o meglio: se ricorre, fa scattare l'interpretazione "investire in".

In concomitanza con entrare, il nesso preposizionale alla Nmc può avere inoltre una funzione diversa, di supplemento locativo (cf. \$3.4.1):

(26) Renato Curcio, lo facevano entrare alla Fiat con la tessera di un compagno e Basone gli fece visitare il reparto presse [...]. (LaR)

In questo brano il nome commerciale ha valore di luogo fisico. 


\subsection{Modificazioni predicative: complementi di specificazione $(\mathrm{Ndi} N \mathrm{Nc})$}

I nessi Prep Nmc considerati in questo paragrafo e nel successivo non rientrano nell'orbita di legittimazione del predicato verbale, come gli esempi delle pagine precedenti, bensì costituiscono la modificazione predicativa di un nome o nesso nominale.

A introdurre i nomi commerciali sono spesso le preposizioni $d i, a, d a$ : preposizioni incolori, che funzionano "come puri relatori, in quanto non contribuiscono, o contribuiscono solo in maniera minima, alla semantica del rapporto fra base e aggiunto" (Jansen 2011). Si tratta quindi di stabilire, da un lato, il tipo di relazione che si manifesta tra nesso nominale e marchionimo e, dall'altro, le proprietà combinatorie dell'insieme, valutando eventuali equivalenze tra i costrutti con preposizione e quelli in cui essa non compare, costituendo variante libera. In questi casi si manifesta con particolare evidenza il valore aggettivale del marchionimo.

I nessi preposizionali introdotti da $d i$ sono manifestazioni formalmente convergenti di una molteplicità di rapporti (cf. La Fauci 2009: 160). In questo paragrafo essi sono analizzati e strutturati sulla base delle loro caratteristiche combinatorie.

Innanzitutto si delineano i casi di complementi di specificazione con valore oggettivo e soggettivo (\$3.2.1). Altri tipi sono poi descritti e differenziati in relazione a presenza o assenza di determinazione davanti al marchionimo e possibile equivalenza con le forme senza preposizione: relazione tra prodotto e produttore (\$3.2.2), tra nome [+ umano] e marchionimo ( $\$ 3.2 .3)$, tra contenente e contenuto (misure di quantità; $\S 3.2 .4)$, tra nome comune e marchionimo con funzione di aggettivo relazionale $(\$ 3.2 .5)$.

\subsubsection{Valore soggettivo e oggettivo}

(1) È con gli anni Trenta, con l'invenzione del Monopoli, primo gioco soggetto a copyright e oggetto di un'autentica attività di promozione e gestione editoriale, che il quadro ha cominciato a cambiare. Per poi evolversi ulteriormente con lo 
sviluppo del merchandising cinematografico che ha fatto dei cartoni Disney un inesauribile modello e fonte per la produzione dei giocattolai, e fino alla nascita di Barbie (1959), vero e proprio giocattolo con storia pre-iscritta o bambola-modello di ruolo. (CORIS MISCRiviste)

In questo brano ricorrono due nessi in cui il marchionimo introdotto da di modifica un nome comune: l'invenzione del Monopoli e la nascita di Barbie. Tanto invenzione quanto nascita sono nomi (deverbali) dalla natura spiccatamente predicativa (cf. La Fauci 2009: 160), correlati a inventare e nascere. Si può pertanto identificare il tipo di relazione sintattica che intercorre tra nome comune e marchionimo modificatore: nel primo caso si parlerà di valore oggettivo del complemento di specificazione ('qualcuno ha inventato il Monopoli'); nel secondo caso di valore soggettivo ('Barbie nasce’) (cf. La Fauci 2009: 161).

Seguono alcuni costrutti in cui la modificazione marchionimica ha valore soggettivo:

(2) Sony non è riuscita a opporsi all'ascesa della Apple con i suo iPod, così come la Playstation ha perso quasi tutto il suo smalto rispetto a Nintendo Wii o Microsoft xBox. (CORIS MON2008_10)

(3) La principale novità per il team al debutto della stagione sportiva imminente [...] sarà proprio la sponsorizzazione di Alitalia, i cui vertici hanno fatto sapere i motivi per cui hanno deciso di puntare su Aprilia Racing [...]. (CORIS MON2008_10)

(4) I lavoratori dell'Alfa Romeo sono tornati ieri in fabbrica dopo una settimana di cassa integrazione e sono stati informati della decisione della Fiat di chiedere lo stato di crisi per accedere alla cassa integrazione straordinaria per un anno. (CoLFIS, quotidiano)

(5) "Siamo favorevoli all'internazionalizzazione e nel caso specifico la sopravvivenza dell'Alfa è legata ad interventi esterni. [...]" (LaR)

(6) L'accordo di Zurigo riavvicina quindi il calcio ai prezzi di mercato. Sarebbe interessante conoscere la reazione dell'Adidas, che su questo finto contenimento dei prezzi ha costruito un impero. (LaR)

Il nesso nominale $N$ di Nmc corrisponde in questi casi a una proposizione $N m c V$. . in cui il marchionimo, in quanto soggetto, sottostà alle restrizioni imposte dal predicato (v. §4.1). Nel caso di la decisione della 
Fiat (4), per es., cui corrisponde paradigmaticamente "la Fiat decide", il marchionimo Fiat si caratterizza come [+ umano].

Nei seguenti esempi la modificazione $d i N m c$ ha invece valore oggettivo:

(7) Non solo a Napoli, ma furti di Rolex gestiti da napoletani sono stati segnalati nel 2006 a Genova, Riccione, Roma. (CORIS MON2005_07)

(8) Si viene così a sapere, per esempio, che i guidatori di Mercedes manifestano la più solida convinzione sulla affidabilità della propria vettura, mentre $i$ possessori di Volvo ne esaltano al massimo le doti di sicurezza, e $i$ conducenti di Toyota sono consapevoli della tecnologia d'avanguardia che distingue la Casa nipponica. (CoLFIS, periodico)

(9) [...] tra le sue abitudini [di Glenn Gould] spiccavano l'isolamento-meditazione nei deserti di ghiaccio oppure la frequentazione degli autogrill per ascoltare il brusio di voci degli avventori, dei camionisti e dei rappresentanti di commercio che vi sostavano. (CoLFIS, periodico)

(10) Leon Brittan ci ripensa e nega il suo consenso al matrimonio del pannolino, annunciato nel settembre '90 da una joint-venture fra i Lines di Ancona e l'americana Procter \& Gamble, produttrice dei Pampers. (LaR)

In questi brani la combinazione di un determinato nome (di carattere predicativo) e di un marchionimo che manifesta funzione di oggetto diretto dà a quest'ultimo un valore specifico: nel nesso $i$ guidatori di Mercedes, per es., tale combinazione fa sì che il nome commerciale sia interpretabile come 'automobile' (per una trattazione a livello proposizionale si rimanda al §4.2). Eppure il marchionimo, in questo tipo di struttura, è probabile ricorra senza articolo, nonostante vi siano studiosi che ritengono obbligatoria la determinazione con il nome d'impresa utilizzato metonimicamente per i suoi prodotti (per es. Vaxelaire 2005: 346). Questo varrà forse in un contesto come quello che si incontra nel seguito dell'articolo: "chi guida una Mercedes sa invece di avere in mano la vettura che si svaluta di meno". Ancora una volta è questione di combinazioni, non di valori assoluti.

Com'è immaginabile, data la natura economica dei marchionimi, sono frequenti i nessi in cui ricorrono vendita, acquisizione e simili: 
(11) [Mitterrand] Ha negato qualsiasi pressione del governo sulle società statali per aiutare Bernard Tapie, ministro delle Aree urbane, nella vendita della Adidas. (CoLFIS, quotidiano)

(12) "Con le finanze che ha a disposizione nella propria tesoreria oltre a quanto incasserà dalla vendita di Galbani, l'Ifil farà fronte alle cifre necessarie per l'aumento di capitale Fiat, per l'acquisto delle obbligazioni convertibili e per l'offerta pubblica d'acquisto su una quota della Rinascente". (CoLFIS, periodico)

(13) In giugno, anche in seguito all'apertura di un'inchiesta per abuso di posizione dominante, Coca-Cola aveva rinunciato all'acquisizione della Cadbury Schweppes. (CORIS STAMPAPeriodici)

(14) La grande sfida sulle acque minerali ha fatto registrare un punto a favore dei francesi della Bsn, con l'acquisto della Ferrarelle. (LaR)

(15) Il Parlamento manifesta tutta la sua sorpresa per la possibile acquisizione dell'Alfa Romeo da parte della americana Ford. (LaR)

(16) Ambrosio era stato arrestato due giorni fa per una vicenda che apre nuovi scenari ai detectives: il pagamento d'una maxitangente, 7,5 miliardi, per una delle prime e più importanti privatizzazioni italiane, l'acquisto della Pavesi da parte della Barilla. (CoLFIS, quotidiano)

I nomi commerciali sono legittimabili dai predicati verbali vendere $\mathrm{e}$ acquistare sia come soggetti, sia come oggetti diretti. Da questo punto di vista, i nessi vendita di Nmc e acquisto di Nmc sono potenzialmente ambigui quanto alla relazione che vi si manifesta. Dai dati si ricava però che $\mathrm{i}$ marchionimi che ricorrono in tali nessi hanno perlopiù valore oggettivo. Negli esempi (15-16) ricorre pure il soggetto logico di acquisto/acquisizione, permettendo così un'interpretazione univoca: "l'acquisto della Pavesi da parte della Barilla" corrisponde paradigmaticamente a "la Barilla acquista la Pavesi", non a "la Pavesi acquista".

Dal momento che lo stesso marchionimo, a seconda dei contesti d'uso, può avere più referenti, la combinazione con alcuni nomi comuni può dare adito a letture diverse, nei termini di una relazione soggettiva od oggettiva:

(17) “[...] I jeans 501, per esempio, sono realizzati con un tessuto prodotto sì in una fabbrica degli Usa, ma esclusivamente per il mercato italiano". Le produzioni di 
Levi's, infatti, avvengono tutte in fabbriche del gruppo, in Europa e negli Usa, con aggiustamenti per ogni mercato nazionale [...]. (LaR)

Non è possibile stabilire univocamente se Levi's sia qui riferito all'azienda, il che fornirebbe un valore soggettivo al complemento di specificazione, $\mathrm{o}-$ più probabilmente $-\mathrm{al}$ prodotto, ai jeans (con un uso del marchionimo non determinato per le ragioni viste nel §1.1.4). Si avrebbe, in questa seconda opzione, un valore oggettivo.

In generale si osserva che la preposizione $d i$, nei nessi con complemento di specificazione a valore oggettivo o soggettivo, non costituisce variante libera. "La nascita Barbie" o "furti Rolex" non sono percepiti come costrutti ben formati. Nondimeno, in alcuni casi si riscontrano casi di $N N m c$ che corrispondono a $N \operatorname{di}($ Det $) ~ N m c$ :

(18) La risposta della Fiat dovrà arrivare entro due delle quattro settimane al termine delle quali dovrà esserci il pronunciamento definitivo sull'offerta della Ford. (LaR)

(19) Un paio di volte Brizio ha alzato, preoccupato, gli occhi dal testo dell'intervento e una è stata quando ha chiesto assicurazioni a Cesare Annibaldi, responsabile delle relazioni esterne Fiat, sul futuro di Mirafiori e Rivalta. La risposta Fiat non si è fatta attendere [...]. (CoLFIS, quotidiano)

(20) "Una scelta - spiegano alla sede torinese - legata anche alla presenza della Lavazza sul mercato francese. La Francia, con un giro d'affari di oltre 200 miliardi, è il mercato più importante, ma anche il più antico, del nostro gruppo". (CORIS STAMPAQuotidiani)

(21) Ufficialmente non è stato neppure individuato un obiettivo di crescita della presenza Lavazza sul mercato transalpino [...]. (CORIS STAMPAQuotidiani)

(22) Lei, Toscani, sputa sulle essenze, ma presto vedremo una campagna per un nuovo prodotto di Benetton: e sarà un profumo. (CoLFIS, quotidiano)

(23) "Benetton e Barilla non vendono prodotti, vendono uno stile di vita. Se stimiamo 100 il valore di un prodotto Benetton possiamo individuare che almeno 50 è rappresentato dalla gestione della rete commerciale, solo 20 è il costo del lavoro di produzione, e il resto è organizzazione, marketing, pubblicità”. (LaR)

In queste coppie di brani, i costrutti con e senza preposizione (articolata) "la risposta (della) Nmc", "la presenza (della) Nmc" e "un prodotto (di) 
Nmc" (sul quale v. §2.2.1) si equivalgono. Nei nessi la risposta Fiat, la presenza Lavazza e un prodotto Benetton, cui corrispondono 'Fiat risponde', 'Lavazza è presente' e 'Benetton produce', i marchionimi si comportano come un tipo specifico di aggettivi di relazione, ovvero "i modificatori che esprimono il soggetto [...] oppure l'oggetto logico [...] del nome a cui si riferiscono" (Ramaglia 2011).

Con riguardo ai rapporti sintattici si può affermare che i nomi commerciali di questi costrutti hanno funzione argomentale (sono argomento - soggetto o oggetto diretto - del predicato espresso tramite il nome comune); invece, nella prospettiva del nesso nominale, è il nome comune a fungere da argomento, mentre il marchionimo (eventualmente preceduto da $d i$ ) ha funzione di modificazione predicativa, come sarebbe per un tradizionale aggettivo. ${ }^{35}$

\subsubsection{Relazione prodotto-produttore}

(1) Dove non arrivavano gli avvocati e i grandi manager, rimediava il mercato nero. I cosmetici della Revlon e della Colgate, il whisky della Seagram, i prodotti della Sony e della Xerox (tutti, a turno, proibitissimi), non sono mai mancati nei suk di molti Paesi arabi. (CoLFIS, quotidiano)

Nei costrutti evidenziati in questo brano il nome commerciale, preceduto dalla preposizione (articolata) di, specifica un nome riferito a un prodotto o lo stesso prodotto (una modificazione a valore soggettivo, come visto nel \$3.2.1). Tra nome e marchionimo si riconosce un rapporto tra opera e artefice (cf. La Fauci 2009: 160) declinato in ottica commerciale: tra prodotto e produttore (o, anche, tra marchio e oggetto marchiato). Non stupisce, pertanto, che tale tipo di modificazione predicativa ricorra di frequente nei dati:

35 Espressioni di forma esplicitamente aggettivale come fiatese, lavazziano o benettoniano (cf. cap. 5) non si trovano se non raramente nel discorso; solitamente, infatti, i marchionimi si presentano come aggettivi invariabili, per ragioni che - si ipotizza - hanno a che fare tanto con il principio di economia linguistica (una stessa forma può ricorrere con più funzioni) quanto con certa reticenza a creare o utilizzare nomi propri modificati morfologicamente. 
(2) Capelli tagliati quasi a zero, braccia e gambe tatuate, vestito con un vecchio paio di scarpe da ginnastica, calzini bianchi, pantaloncini neri della Adidas e maglietta grigia (tutto sdrucito e sporco) il re degli hooligans forse sapeva di non essere un tipo da passare inosservato. $(\mathrm{LaR})^{36}$

(3) La conclusione? Che il 2010 è stato l'anno dell'iPad - ma già lo dicevano le cifre, il tablet della Apple è uscito in aprile e ha una media di 1,3 milioni di unità vendute al mese. (CORIS MON2008_10)

(4) Shopping per innamorati: gioielli, lingerie, gadget, libri a suon di baci, viaggi e l'orologio della Swatch. (LaR)

(5) Oggi l'azienda fa capo a un'unica società, la Fiat Spa, che raggruppa il controllo di tutte le attività industriali: le automobili con i vari marchi, $i$ camion dell'Iveco, i trattori e i macchinari per le costruzioni prodotti dalla controllata Case New Holland (Cnh). (CORIS MON2008_10)

(6) Lo straniero che arriva a Mosca [...] resta inevitabilmente colpito e sorpreso [...] dalle russe di mezza età con borsa di Vuitton che fanno la spesa da Sadko, il supermarket $[\ldots]$. (LaR)

(7) Bisogna fare i conti con una società molto puritana, che ha "bocciato", per esempio, la campagna pubblicitaria dei jeans di Calvin Klein, troppo osé e allusiva. (CoLFIS, periodico)

La struttura è sempre la medesima: un nesso nominale riferito a un prodotto, seguito dalla preposizione $d i$ e da un nome commerciale il cui settore di produzione comprende il tipo di oggetto designato dal nome comune. Una possibile variazione concerne la determinazione: come mostrano gli esempi (6) e (7), l'articolo è tendenzialmente assente con marchionimi che conservano un forte carattere di antroponimi, come le griffe Vuitton e Calvin Klein. ${ }^{37}$

36 Si noti, in questo esempio e nel successivo, la mancata elisione dell'articolo, per niente rara davanti a marchionimi inizianti per $A$ (non ci risulta che ciò sia mai stato osservato in relazione ai nomi commerciali). Nel caso di "(la) Aia" questo avviene in maniera pressoché totale, come mostrano le ricorrenze del corpus LaR; la non-elisione pare imporsi pure per la funzione disambiguante rispetto al poleonimo L'Aia.

37 Tale assenza si riscontra in maniera sistematica con firme dell'alta moda anche nei romanzi di Moccia e Brizzi (v. cap. 7): “jeans di Missoni”" (Brizzi 1994: 30); "il vestito di Valentino" (Moccia 2004: 55 e 63); "la cinta doppia di Camomilla" (Moccia 2004: 122). La preposizione è invece articolata negli altri casi 
Ricorrono come nomi modificati anche marchi (registrati) di un prodotto specifico:

(8) $[\ldots]$ una ricerca di Accenture [...] mostra come la gran parte degli italiani sia frustrata dalla complessità e abbia desiderio di un prodotto in grado di svolgere un solo compito. In questo senso l'iPod di Apple (semplice anche nel design) ha fatto scuola. (CORIS MON2005_07)

(9) Nel frattempo i produttori [di detersivi] cercano di conquistare la leadership sul mercato ricorrendo a tutte le armi permesse dalla pubblicità. A contendersi il primato sono il "Dash" della Procter e il "Dixan" della Henkel attestate sul 23-24 per cento a testa. (LaR)

(10) Sono sette le nuove Salsallegre della Kraft, studiate per dare un sapore in più alla carne o al pesce. (CoLFIS, periodico)

(11) "Per gli Italiani - spiega il quotidiano - le Timberland a cento dollari, $i$ Crono [sic] della Swatch a ottanta e i blue jeans della Levi's a quaranta sono occasioni eccezionali. [...]”. (CoLFIS, quotidiano)

Alcune combinazioni di nome comune e marchionimo ricorrono senza che quest'ultimo sia preceduto dalla preposizione, con valore equivalente (cf. supra, §2.2.1):

(12) Alessandro che fa il gelataio. Fabio che non lavora: "Niente, io non faccio niente". Ha il berretto di lana, scarpe e pantaloni Adidas, una sciarpa al collo. $(\mathrm{LaR})$

(13) La commercializzazione delle Olimpiadi raggiunge livelli mai visti, ma al tempo stesso consente, per esempio, la raccolta di fondi per la ricostruzione dello stadio di Sarajevo attraverso le royalties di un orologio Swatch. (LaR)

(14) Appena al di là delle Alpi, nello stabilimento francese di Bourbon-Lancy dove si producono motori per $i$ camion Iveco, il lavoro costa il 15 per cento in meno. (LaR)

(15) Sparite le borse Vuitton, anche i ricchi team manager girano con la sacchetta di plastica da supermercato. (CoLFIS, quotidiano)

$N$ di Nmc, che sono la maggioranza: "il supercronografo della Swatch" (Moccia 1992: 98); "la maglietta nera, elasticizzata della Onyx" (Moccia 2004: 12); "la crostatina del mulino bianco" (Brizzi 1994: 49). 
In un'ottica funzionale il rapporto tra i due componenti del nesso - del tipo "prodotto-produttore" - è comparabile a quello individuato per la struttura $N d i$ (Det) $N m c$. In entrambi i nessi i nomi commerciali rivestono funzione attributiva rispetto al nome che precede. La variazione libera della preposizione $d i$ in questo tipo di strutture è un aspetto caratteristico dei marchionimi, in grado di fungere da aggettivi restando formalmente invariati; nomi propri e comuni non lo presentano, ricorrendo di norma preceduti dalla preposizione ("un quartetto di Schubert", " $i$ dipinti di Chagall").

\subsubsection{Con nomi [+ umani]}

(1) "Nella crisi", riassume Herbert Hainer, presidente di Adidas, "il modello tedesco s'è dimostrato il migliore". (CORIS MON2008_10)

(2) Lo ha annunciato il presidente della Coop, Ivano Barberini, nel corso della conferenza stampa per la presentazione del nono congresso dell'associazione. $(\mathrm{LaR})$

(3) L'iniziativa è stata presentata ieri a Bollate presso il centro di ricerca e sperimentazione Csi da Giancarlo Cimoli, amministratore delegato di Edison alla presenza del ministro dell'Ambiente Giorgio Ruffolo. (CoLFIS, quotidiano)

(4) L'idea che l'avvenire possa dipendere da Washington o Londra crea uno stato di sofferenza acuta nell'operaio della Nissan o nel venditore di spiedini di pollo a Shibuya. (LaR)

(5) "Abbiamo dei manualetti con avvertimenti generici: riposare prima di un lungo viaggio e, dopo l'atterraggio, mangiare proteine se si deve stare svegli, carboidrati se si va a dormire" racconta Gerardo Lettich, comandante dell'Alitalia che viaggia da anni lungo le rotte intercontinentali. (CoLFIS, periodico)

(6) È morto sabato scorso a Milano, all'età di 92 anni, Giovanbattista Guidotti, capo collaudatore e pilota dell'Alfa Romeo per un cinquantennio. (CoLFIS, quotidiano)

(7) La Delta Integrale, a causa della linea più squadrata, è nettamente meno filante della rivale tedesca, una caratteristica questa però ritenuta poco importante dai tecnici della Lancia per una vettura nata espressamente per i rally [...]. (CoLFIS, periodico) 
(8) «Solo i prodotti di marca investono nell'innovazione», afferma Nicole Bugnon, portavoce di Henniez. (SM 6/05)

In questi brani il nesso nominale modificato dal nesso preposizionale designa un essere umano. Di frequente esso indica un ruolo professionale svolto all'interno dell'impresa (presidente, amministratore delegato, operaio) o la professione specifica (capo collaudatore e pilota). Dal punto di vista testuale si osserva che spesso il nesso $N$ [+hum] $\operatorname{di}($ Det $) \mathrm{Nmc}$, in particolare quando ricorre al singolare, si accompagna a un antroponimo, rispetto al quale si pone come apposizione interna o esterna.

Non mancano inoltre usi come il seguente:

(9) Dopo vent'anni di galera al Cairo per traffico di droga rilasciano Fioravante Palestini, che se non avesse fatto il martellatore della Plasmon non se lo sarebbe filato nessuno. (CORIS MON2001_04)

Martellatore non è una professione stricto $s e n s u^{38}$ (il riferimento è qui a una pubblicità di Plasmon degli anni Sessanta) ma funziona sintatticamente come tale.

L'alternanza tra strutture con e senza preposizione (cf. supra, $\S 2.2 .3)$ si riscontra anche per questa struttura, all'interno dello stesso articolo (10) o in testi diversi: ${ }^{39}$

(10) Una buona bandiera per il partito dei lavoratori, la candidatura di Alvaro Superchi, 49 anni, ex muratore e operaio Alfa dal 1972, segretario di sezione e delegato del consiglio di fabbrica per la CGIL. [...] In una riunione della federazione, alla quale ho partecipato anch'io, si era deciso che un operaio dell'Alfa ci sarebbe dovuto essere tra i candidati nei collegi uninominali. (CoLFIS, quotidiano)

(11) Concorda il presidente Coop Aldo Soldi: «Il successo è stato evidente fin dall'inizio. [...]». (CORIS MON2008_10)

38 Si confrontino i costrutti ha fatto l'operaio alla Fiat e 'ha fatto il martellatore alla Plasmon.

39 Nel caso di nomi [+ umani] che non designano una professione, per es. "il fondatore della Kodak, George Eastman" (CORIS STAMPASupplementi), sembra più probabile il costrutto con preposizione. 
(12) Nel leggere la relazione scritta, il dottor Pieri ha aggiunto due aggettivi che non comparivano nel testo distribuito al pubblico: interferenza politico-sindacale. Un'aggiunta che non è passata inosservata al principale imputato di quel processo, l'amministratore delegato Fiat Cesare Romiti, seduto in seconda fila tra le autorità. $(\mathrm{LaR})$

(13) Ruggero Gorgoglione, 25 anni, operaio Fiat e Maria Rosaria Triola, 24 anni, casalinga, via Lulli 3, entrambi incensurati, stavano circondando di attenzioni la loro bambina di 2 anni, che portavano a fare una passeggiata. (CoLFIS, quotidiano)

(14) Cadalora certamente è il favorito, ma penso che si tratterà di un campionato assai combattuto e potrà accadere che a contendergli il titolo sino all'ultimo possa essere un pilota Aprilia. (CoLFIS, periodico)

(15) Coop, giunta al quarto posto, critica il concetto stesso di confronto. «Dovrebbe comprendere numerosi altri fattori» dichiara Marco Scossa, portavoce Coop Regione Ticino. (SM 4/05)

La possibilità di utilizzare senz'altro il marchionimo come modificatore del nome - in particolare quando il costrutto N Nmc funge da apposizione - ne mette in luce, una volta di più, il carattere intrinsecamente attributivo.

Il nesso di(Det) $N m c$ ricorre come modificatore dello stesso uomini:

(16) Una discussione fra Senna, Frank Williams e i tecnici della Goodyear - avvenuta durante il pranzo del sabato che ha preceduto la gara di Imola - offre una chiave di lettura nuova per fare luce sulle cause dell'incidente che è costato la vita al tre volte campione del mondo. [...] Il capo della scuderia e gli uomini della Goodyear ascoltano lo sfogo del campione. (CoLFIS, quotidiano)

(17) Come fare a invertire la rotta e nel contempo a zittire gli irriducibili nostalgici del Drake Enzo Ferrari, quelli che dicono che da quando è morto il vecchio e a comandare sono gli uomini della Fiat l'azienda non è più la stessa? (CoLFIS, periodico)

Nel brano (16) "gli uomini della Goodyear" è ripresa anaforica di "i tecnici della Goodyear" che ricorre poche righe prima. Nel caso di (17), "gli uomini della Fiat", il riferimento sarà piuttosto ai manager di Fiat. 
Con questo valore specifico ricorre alcune volte, nei dati, la variante senza preposizione uomo/uomini Fiat (cf. §2.2.3): ${ }^{40}$

(18) Gli uomini Fiat arrivano, confessano, e subito dopo partono gli arresti verso i destinatari delle tangenti: come è già accaduto all'Asst, l'azienda dei telefoni di stato. E gli uomini Fiat se ne vanno, accompagnati dai loro avvocati, indagati ma a piede libero. Finora Cesare Romiti è stato l'unico uomo Fiat - tra quelli passati in Procura - a vedersi riconoscere lo status di "persona informata sui fatti", cioè testimone. (CoLFIS, quotidiano)

Nei brani riportati, uomo/uomini (della) Fiat - in particolare nella variante senza preposizione - ha subíto una sorta di specializzazione semantica che non ricorre con altri nomi commerciali (per es. Goodyear), quantomeno in riferimento ai dati analizzati.

Concludiamo con un'altra struttura $N[+h u m] d i N m c$, nella quale il nesso preposizionale modifica un nome di nazionalità:

(19) Ma noi non aumenteremo i prezzi. Gli svizzeri della Swatch, con grande soddisfazione dei collezionisti italiani (a detta dei più tra i più grandi "consumatori" al mondo dei famosi orologini di plastica) hanno deciso di non rilanciare. (LaR)

(20) Il consorzio a cui hanno aderito gli svizzeri di Migros, i francesi di Carrefour, $i$ belgi di Delhaize, gli irlandesi di Superquinn oltre ai gruppi inglesi Sainsbury's e Marks \& Spencer costituisce di fatto una vera e propria lobby industriale [...]. (LaR)

Il costrutto, in apparenza simile ai precedenti portavoce di Henniez e operaio della Nissan (tra il resto prevede anch'esso il ricorrere di articolo davanti al marchionimo come variante libera), in realtà se ne differenzia per la natura sintattica delle relazioni tra nome comune e nome commerciale. È quest'ultimo, infatti, a costituire il nucleo funzionale di costrutti quali gli svizzeri della Swatch, rispetto al quale l'aggettivo nominalizzato svizzeri, francesi ecc. funziona come modificazione di tipo non-restrittivo: una perifrasi appropriata sarebbe "quelli della Swatch, che sono svizzeri" o "la Swatch, che è svizzera". Si osservi, del resto, che il costrutto ricorre nei dati con il nome comune al plurale, mentre per le altre strutture viste sin qui si danno anche (o soprattutto) casi al singolare.

$40 \quad$ Per il valore di uomo e uomini si veda inoltre il titolo dell'articolo dal quale è estratto il brano (18): "Scontro di manager, Romiti sotto tiro". 


\subsubsection{Relazione contenente-contenuto}

(1) “Può darmi un po' d'acqua? Sono due giorni che non bevo" mi ha chiesto [Fabio Tacchinardi]. Gli ho buttato una bottiglia di Ferrarelle e gli ho domandato dove lo tenevano prigioniero. (LaR)

La specificazione di Nmc modifica, in (1), un nesso nominale indicante un contenitore (e una quantità): una bottiglia. Similmente avviene nei casi seguenti con lattine, casse, pacchetto ecc.: ${ }^{41}$

(2) Anche l'opera contemporanea ha toccato il prezzo maggiore a livello internazionale: "210 bottiglie di CocaCola" di Andy Warhol è stato venduto a New York per oltre 2 milioni di dollari. (CoLFIS, quotidiano)

(3) " $[\ldots]$ io credo che bisogna anche attribuire alla gente le responsabilità che le spettano: non c'è senso civico, tutti si comportano come barboni, i parchi sono pieni di lattine di Coca Cola, di pacchetti di sigarette vuoti”. (LaR)

(4) Al contrario, nella tazzina di Lavazza bevuta all'estero si troverà, fanno capire i pubblicitari, la sferzata necessaria a lavorare di più [...]. (LaR)

(5) 'Prego, poggi pure lì. Quante sono le casse di Evian? E quelle di Ferrarelle? E soprattutto: quant'è? Bene ragazzi, è arrivata l'acqua minerale. Sotto con la colletta, c'è da pagare il conto'. (LaR)

(6) [I consumatori] sono tutti molto competenti sui prezzi: una qualunque brava massaia ricorda a memoria quanto costa il pacchetto di Lavazza Rossa (il leader di mercato) e si accorge immediatamente di ogni rincaro. (LaR)

(7) L'unico commercio - gratis, per ora - è quello della militaria lasciata dai serbi. Per una targa in cirillico si offrono tre pacchetti di Marlboro. (LaR)

(8) L'erede all'inesistente trono d'Italia è seduto nel suo studio in Chemin des Princes, nella banlieue elegante di Ginevra. Abbronzatissimo, è in maniche di camicia con la sigla ricamata a mano sormontata da una piccola corona e porta un paio di Levis bianchi. (LaR)

(9) [Drew Barrymore] Arriva nella hall della clinica, dove l'aspetta Todd Gold, consumato specialista di questo genere di storie, con addosso un paio di jeans e una maglietta, in mano una scatola di kleenex. (CoLFIS, periodico)

41 Cf. anche "Fino a qualche tempo fa ci si preparava tra bagno e guardaroba, un colpo di rimmel e uno di spazzola" (CORIS MON2005_07). 
(10) Qualche dolore nei giorni successivi va considerato del tutto normale, se non riuscite a tollerarlo potete trovare giovamento in una compressa di aspirina (sempre che non sussistano controindicazioni mediche al prodotto) da ingerire a stomaco pieno [...]. (CoLFIS, periodico)

Il rapporto tra nome modificato e marchionimo non è lo stesso che si registra nei casi visti sin qui, da jeans di Calvin Klein a operaio della Fiat. Una prova, in prospettiva sintattica, è data dal fatto che le strutture con un nome relativo a un contenente non hanno corrispondenza funzionale con il costrutto $N N m c$ senza preposizione. Non è nemmeno possibile, generalmente, integrare un determinatore davanti al marchionimo, che invece ricorre spesso nelle strutture analizzate nei paragrafi precedenti, e ciò a prescindere dal suo valore originario di 'impresa' (Ferrarelle, Marlboro) o di 'prodotto' (kleenex, aspirina). ${ }^{42}$

Benché superficialmente simile ai costrutti visti sinora, dunque, nel nesso indicante una relazione contenente-contenuto il marchionimo presenta caratteristiche combinatorie diverse. Esso costituisce il nucleo del nesso nominale e non, come negli altri casi, la sua modificazione, la quale spetta invece al nome indicante un contenitore o una misura di quantità.

Un'eccezione apparente a quanto sin qui osservato è data da $i l$ fustino (di) Dash, che nei dati ricorre nelle tre varianti con preposizione, articolata o no, e senza preposizione:

(11) A Pisa son passati tredici allenatori in otto anni, ma Guerini è da tenere stretto come il fustino di Dash. (LaR)

(12) Ho conosciuto - ad esempio - il famoso pittore Margutti: non scambierei con lui, non dico opinioni, ma neppure il fustino del Dash. (LaR)

(13) Cragnotti vorrebbe ancora di più perché sa benissimo che con l'acquisto della Mira Lanza ( 8 per cento del mercato italiano dei detersivi) la Henkel riempirebbe strategicamente dei vuoti e diventerebbe leader assoluto battendo l'americana Procter and Gamble che produce, fra l'altro, il fustino Dash. (LaR)

42 Quando i nomi commerciali di questa struttura sono (originariamente) riferiti all'impresa, si tratta di usi metonimici per il prodotto $(\S 1.1 .3)$ : "tre pacchetti di Marlboro" corrisponde a "tre pacchetti di sigarette Marlboro", un tipo di costrutto che ricorre nei dati con minore frequenza (nel corpus LaR vi è una sola ricorrenza di "pacchetto/-i di sigarette Marlboro", di contro a 37 casi di "pacchetto/-i di Marlboro"). 
(14) Quelli bravi invece sono i veterani della Tv, non sopraffatti dal loro sapere: è bravo Maurizio Costanzo, è bravo Mike Bongiorno che fa il suo lavoro con serietà imperturbabile anche se succede il finimondo, che tiene tra le braccia $i l$ fustino Dash come se fosse un bambino piccolo da amare. (LaR)

Negli esempi (13-14), peraltro un caso isolato, il marchionimo assume valore qualificativo.

Si conclude con un brano solo in parte differente da quelli sinora approfonditi:

(15) Michelangelo Detoni era un manutentore come Nevio Manzan, classe 1931, un omino smilzo che si è fatto 26 anni di Michelin. (CORIS STAMPAQuotidiani)

Per il costrutto "si è fatto 26 anni di Michelin" si può parlare di quantità temporale; per il resto i caratteri formali e combinatorî sono gli stessi riscontrati per il tipo "contenente-contenuto", in particolare l'assenza di determinazione e l'impossibilità di tralasciare la preposizione.

\subsubsection{Funzione di aggettivi relazionali}

(1) "Trenta anni, è il primo Agnelli della quarta generazione ad essere eletto nel board della Fiat. Ed è anche chairman della Piaggio Veicoli Europei”. Ma il posto dello zio non è automatico - precisa [la rivista] $\mathrm{W}$ - perché "un nuovo gruppo di azionisti esterni è entrata [sic] nel board Fiat". (CoLFIS, quotidiano)

Il costrutto board della Fiat, con complemento di specificazione, trova un corrispondente funzionale nel nesso board Fiat evidenziato nel seguito, ossia una modificazione attributiva che assume le fattispecie di un aggettivo relazionale (cf. La Fauci 2009: 154 e 160; Ramaglia 2011). In entrambi i casi, infatti, il rapporto tra nome comune board e nome commerciale Fiat non è di tipo qualificativo, con un attributo che denoti una proprietà, bensì di relazione tra il nome modificato e il nome da cui l'aggettivo è derivato (cf. Ramaglia 2011), come sarebbe per il nesso il sistema solare (con l'equivalente il sistema del sole) rispetto a una ragazza solare (cui non corrisponde una ragazza del sole). Gli aggettivi relazionali sono derivati da nomi (cf. Ramaglia 2011); nel caso dei marchionimi, lo si è già visto, la derivazione è il più delle volte una conversione funzionale senza manifestazione a livello di forma. 
Lo stesso si ritrova nella seguente coppia di esempi:

(2) L'obiettivo della Fiat, nel quadro di una strategia globale che ovviamente continua a porre l'Europa in primo piano, è di conquistare nuovi spazi in questo difficile mercato e di consolidare i risultati conseguiti. (CORIS STAMPAQuotidiani)

(3) L'obiettivo Fiat è di proseguire sulla strada dell'espansione per arrivare agli anni Novanta con un fatturato di 50 mila miliardi. (LaR)

Si tratta di una relazione caratterizzabile in termini di possesso: Fiat, si potrebbe affermare, ha un obiettivo. Non così nel brano (4):

(4) I francesi della Bsn, leader di mercato, hanno deciso di puntare soprattutto su due marchi, Boario e Ferrarelle [...]. Per Boario la volontà è di trasformarla da marca locale a marca nazionale. L'obiettivo Ferrarelle è di svilupparne le esportazioni contando sulle particolari caratteristiche. (LaR)

Qui non c'è relazione di possesso tra Ferrarelle e obiettivo: è questione, infatti, dell'obiettivo di Bsn in merito a Ferrarelle, non dell'obiettivo che ha Ferrarelle. Per Bsn, in sostanza, Ferrarelle è l'obiettivo.

Parallelamente ai casi analizzati sinora, pure i nessi di(Det) $\mathrm{Nmc}$ negli esempi seguenti funzionano come aggettivi relazionali:

(5) La novità più visibile riguarda la barra degli strumenti, adattabile alle esigenze dell'utente (icone che si possono eliminare, altre che si possono aggiungere) e un disegno più accattivante, che ricorda quella di Mac OS X, il sistema operativo di Apple. (CORIS MON2008_10)

(6) "[...] Non condividiamo la politica di Alitalia, che mette da parte Malpensa a vantaggio di Fiumicino". (CoLFIS, quotidiano)

(7) $[\ldots]$ proprio ieri sera la Federazione internazionale ha riconosciuto come valide le tesi dell'Agip e come regolare l'ultimo sviluppo di benzina (sigla 110) che la Ferrari deciderà oggi se utilizzare. (CoLFIS, quotidiano)

(8) Per la verità le condizioni di prevenzione della salute nello stabilimento della Lavazza sono apparse nel corso dell'inchiesta piuttosto buone. (LaR)

La modificazione attributiva esprime, anche in questi casi, una relazione di possesso. 
La corrispondenza tra complemento del nome (con di) e marchionimo in funzione predicativa è spesso possibile, come si osserva in queste coppie di esempi:

(9) Il presidente della Fiat, Cesare Romiti, transita turbato davanti allo stand della Nutella, indeciso se farsi accalappiare dalla mistura calorica. (CORIS Stampa Quotidiani)

(10) [...] per i golosi il top è lo stand Nutella con degustazione di crepes con la famosa crema nocciola cacao. (CORIS Stampa Quot)

(11) Interessante lo sbocco commerciale: col marchio "Clementine della valle di Sibari”, questi mandaranci sono stati distribuiti l'anno scorso sugli aerei dell'Alitalia; e 30 mila, in confezioni singole, sono finiti sulla flotta Lufthansa. (CoLFIS, periodico)

(12) I treni sono tornati a viaggiare, ma adesso sono gli aerei Alitalia che si fermano. La staffetta degli scioperi prosegue. (LaR)

(13) L'ultima tessera l'ha presa ieri Natalina, la novantenne cameriera di Manfredi negli spot della Lavazza. E chi meglio di lei poteva dire che il partito radicale più lo mandi giù, più ti tira su? (LaR)

(14) Ben oltre il diretto riferimento agli spot Campari e Barilla da lui girati negli anni Ottanta, in assoluta prossimità al "manifesto" Ginger e Fred, Fellini risulta intriso di una cultura di cui la pubblicità è un elemento essenziale. (CORIS PRACCVolumi)

Negli esempi a seguire la modificazione predicativa costituita dal marchionimo Lego ricorre in costrutti diversi per forma, ma tutti funzionalmente equivalenti:

(15) I mattoncini Lego, inventati nel 1932 da un carpentiere danese, negli anni cinquanta divennero un successo mondiale. (LaR)

(16) 58 milioni di persone fanno l'Italia. 58 milioni di mattoncini di Lego fanno Legoland, il parco dedicato a uno dei giochi più semplici e belli del mondo. (CORIS MON2008_10)

(17) Caserme dei pompieri, vere caserme dei pompieri, che sembrano costruite con $i$ mattoncini del Lego e la facciata del deposito, bianca a macchie nere, è il mantello di un cane dalmata della Carica dei 101. (LaR) 
(18) Eppure $i$ mattoncini della Lego sono riconosciuti dalle autorità pedagogiche di tutto il mondo come utili alla crescita e all'equilibrio psicologico dei più piccini [...]. (LaR)

In tutti questi casi - mattoncini Lego, di Lego, del Lego, della Lego -, indipendentemente da variazioni formali, il nome commerciale ha funzione di aggettivo relazionale.

Non in tutti i complementi di specificazione, peraltro, il marchionimo svolge una funzione comparabile a quella dell'aggettivo relazionale:

(19) E la colonizzazione della ristorazione collettiva veloce e sterilizzata, monosapore e multicolore è soltanto all'inizio. Il colosso americano della McDonald ieri ha presentato il suo primo tempio della polpetta ma i tempi sono acceleratissimi. (LaR)

McDonald costituisce in (19) il nucleo del nesso nominale, non la sua modificazione (come per $i$ belgi di Delhaize, §3.2.3), ed è pertanto funzionalmente comparabile a un'apposizione (cf. §1.3.2), come sarebbe "il colosso americano McDonald".

\subsection{Altre tipologie di modificazioni predicative: il costrutto $N$ da $N m c$}

I nessi preposizionali con marchionimi introdotti da $d i$ non sono gli unici a fungere da modificatori. Si delineano qui altre strutture, in cui ricorre la preposizione $d a$.

(1) Aiuto regista di Camillo Mastrocinque, Angeli esordisce nel mondo della pubblicità nel ' 57 per dare una mano a Luciano Emmer con un Carosello per il dentifricio Colgate, con Lilly Cerasoli, modella bella e simpatica, ogni volta alle prese con avventure tremende, gli indiani la minacciano, la legione straniera la rapisce. Si salverà con un sorriso, un sorriso da Colgate. (CORIS STAMPA Quotidiani)

(2) Il che vuol dire che quasi la metà dei manager, dei dirigenti, in generale di tutti i professionisti che sempre più spesso lavorano «in mobilità» non può fare a 
meno di portarsi dietro il Blackberry. Molto meno certo è invece che il «pollice da Blackberry» sia soltanto una leggenda. (CORIS MON2008_10)

(3) [..] gli orologi Swatch, che da vero e proprio fenomeno sociale sono oggetto di speculazione da parte dei collezionisti. Nei negozi non si trovano più, oppure hanno prezzi proibitivi. Gli esemplari sono pochi e la febbre da Swatch si scatena. (CoLFIS, periodico)

(4) La febbre da Playstation, anzi da Ps 2, la nuova console per videogames della Sony messa in vendita oggi per la prima volta in Giappone (nel resto del mondo arriverà solo dopo l'estate) ha contagiato migliaia di giovanissimi "gamer" [...]. $(\mathrm{LaR})$

In questi esempi, il complemento da Nmc si contraddistingue per l'assenza rigorosa del determinatore davanti al nome commerciale. ${ }^{43}$ Esso modifica un nome comune, con il quale il marchionimo instaura una relazione che si può definire causale.

I brani che seguono presentano aspetti diversi del costrutto:

(5) A condurre è Milly Carlucci, che ha messo in naftalina il suo look da Barbie lanciandosi in un taglio corto alla Ombretta Fumagalli. (LaR)

(6) Gianfranco Funari - Filosofia da Baci Perugina o da siparietti di Dapporto, sorriso da imbonitore, gesticolazione da portiere di Jolly Hotel, più Upim che Rinascente, conflittualità da trattoria. Voto $0(\mathrm{LaR})$

“[La lettera di Epicuro a Meneceo] È un'opera dalla limpidezza involontariamente umoristica", dice [Alberto Bevilacqua]. "Mi sembrano frasi fatte apposta per incartare i cioccolatini degli dei. Si dicono cose di un'ovvietà assoluta". "Scrittore da baci Perugina sarà lui, non Epicuro", è la replica secca di Baraghini. (LaR)

Si entra qui nel campo degli usi allusivi o traslati del nome commerciale, che saranno approfonditi in seguito (cap. 6). Questi usi dei marchionimi Barbie e Baci Perugina ne rivelano un contenuto predicativo che, basandosi sul riferimento a un prodotto e al contempo travalicandolo, viene riferito a un nome comune correlato: l'allusione a un determinato look in correlazione con Barbie; il riferimento allo stile popolar-divulgativo

43 Per opposizione si veda il caso di "un decennio - gli Anni Ottanta - di follie finanziarie con tanto di yuppies dai Rolex d'oro" (CORIS STAMPAQuotidiani). Qui la preposizione $d a$, oltre a essere articolata, è paradigmaticamente equivalente a con; non si riscontra un rapporto di tipo causale. 
delle massime scritte sui cartigli che avvolgono i Baci Perugina, nei casi di filosofia e scrittore.

\subsection{Supplementi predicativi}

Dopo aver considerato i complementi, di nome o di verbo, introdotti da una preposizione, in questo paragrafo è la volta dei supplementi predicativi (La Fauci 2009: 195-211) o complementi avverbiali, che si distinguono dai nessi analizzati sinora in quanto si situano oltre i limiti della proposizione semplice, rispetto alla quale hanno meno restrizioni di posizione.

\subsubsection{Supplementi locativi}

Entro l'insieme dei supplementi predicativi con marchionimo, i supplementi locativi (cf. La Fauci 2009: 206) fanno la parte del leone. Questo non stupisce, considerato che il valore di luogo fisico è uno dei principali per i nomi commerciali riferibili a un'impresa, insieme a quello di persona giuridica o "umano collettivo" (cf. Grass 2002: 228).

(1) Negli Stati Uniti il cavallo dell'economia ha ripreso a bere, ma dalle fabbriche di Detroit, se tutto va bene, si passa a imbottire panini da McDonald, si accettano salari peggiori. (CoLFIS, quotidiano)

(2) La pressione di quella cultura pauperistica fu tale che persino in Fiat a qualcuno venne in mente che si sarebbero dovute smontare le catene di montaggio per dedicarsi ad altro. (CoLFIS, periodico)

(3) All'Ikea, un set di 20 pezzi di diverse forme e misure, di marca Pruta, costa solo fr. 7.90. (SM 3/05)

I supplementi locativi di questi esempi sono identificabili, tradizionalmente, come casi di stato in luogo. Già in questo minimo campione si può osservare la varietà di soluzioni adottate quanto alla preposizione. 
Nessi locativi legittimati da in vendita, come se ne trovano numerosi nel corpus di Spendere Meglio, ricorrono con le preposizioni $d a, a$ (articolato) e presso:

(4) Bonny, in vendita da Ikea a fr. 79.-, è arrivata prima in classifica nonostante il laboratorio abbia criticato uno spigolo che teoricamente potrebbe portare a una rottura prematura della sedia. (SM 4/07)

(5) Il prodotto per la pulizia dei piatti di marca Handy Matic, in vendita alla Migros, costa nel formato tradizionale in polvere fr. 3.60 ed è sufficiente per 30 lavaggi. (SM 4/05)

(6) Sull'impugnatura di Gillette Power vanno anche bene le più economiche lamette Turbo, che sono in vendita ad esempio presso Denner al $20 \%$ in meno dei concorrenti. (SM 2/06)

In combinazione con predicati quali acquistare o costare prevalgono nei dati le preposizioni $d a$ e presso, ma ricorre anche $a+$ articolo:

(7) Pochissimi gli stilisti che comprano cose non griffate da loro. Eccezioni: Dolce e Gabbana a volte acquistano da Fiorucci [...]. (CORIS STAMPAPeriodici)

(8) Fino ad ora, i 1'949 contenitori in plastica brevettati si potevano acquistare solo da Tupperware. (SM 3/05)

(9) Migros e Coop sono risultate quasi alla pari. Su 40 prodotti, 8 costavano meno da Migros, mentre altri 8 costavano meno da Coop. (SM 2/06)

(10) Chi acquista presso Migros il piatto precotto Tradition Casimir Suisse di Anna's Best non si gusta nessun pollo indigeno. (SM 4/07)

(11) Presso Coop, queste lampadine costano molto di più, ma la politica dei prezzi non cambia. (SM 1/07)

(12) Esempio: un libro italiano di 10 euro costa fr. 15.- alla Migros e fr. 17.- alla Melisa. (SM 4/03)

In queste strutture la variazione tra le preposizioni da/a e presso, utilizzate con valori equivalenti, sembra vada ricondotta a una motivazione interlinguistica. Presso come introduttore di nomi commerciali, 
infatti, ricorre praticamente solo nei dati svizzeri di Spendere Meglio ${ }^{44}$ dal momento che gli articoli della rivista ticinese sono spesso traduzioni dal tedesco, si può supporre che presso $\mathrm{Nmc}$ sia influenzato dalla struttura tedesca bei Nmc. ${ }^{45}$

La variazione tra $a$ e $d a$, invece, può essere correlata a fattori sintattici:

(13) Un chilo di sale all'Iper di Grandate o al Carrefour di Como costa 15 centesimi. In Svizzera, pochi chilometri più a nord, il prezzo è oltre sei volte superiore. Non sempre però fare la spesa in Italia è così conveniente: all'Aldi di Chiasso un chilo di tonno costa fr. 6.10, al Bennet di Montano Lucino oltre il doppio: fr. 14.12 (SM 4/10)

Nei nessi evidenziati, $a+$ articolo non è sostituibile con $d a$, come invece sarebbe possibile negli esempi citati sopra. Dal momento che ricorre una specificazione (locativa) del marchionimo, "all'Iper di Grandate", la soluzione con preposizione articolata alla sembra l'unica effettivamente possibile.

Meno usuale è la preposizione in combinata a un predicato quale vendere:

(14) Concorda il presidente Coop Aldo Soldi: «[...] L'incidenza su tutto il pane venduto nelle nostre Coop è stata dell' $8 \%$ lo scorso anno, ma stiamo superando $1 ' 11 \%$ in questo primo scorcio del 2008». (CORIS MON2008_10)

Sul ricorrere di in influiscono tanto il valore del marchionimo Coop, tendente al nome comune (si noti l'uso al plurale), quanto la presenza dell'aggettivo possessivo.

Nei costrutti visti sin qui l'uso di diverse preposizioni con valore locativo ha un risvolto concretamente morfosintattico e uno, più sottile, di tipo categoriale. Quanto al primo punto: soltanto $a$ prevede il

44 Nel resto dei dati ricorre solo con nomi di case editrici, nel costrutto "edito presso $N$ ". In generale, presso è poco usato nei dati di provenienza italiana, mentre quelli tratti da Spendere Meglio presentano frequenze ben più alte, soprattutto in combinazione con marchionimi.

45 Per es. "Wer bei Migros oder Coop ein Erdbeerjoghurt kauft, zahlt bei beiden Grossverteilern im günstigsten Fall 42 Rappen pro Becher" (K-Tipp 08/15; $<$ www.saldo.ch/artikel/d/hohe-preise-fuer-oft-unnoetige-lebensmittel/>, 1.6.2015). 
ricorrere di un determinatore davanti al nome commerciale; si tratta di una presenza necessaria, combinatoriamente condizionata. Ne consegue che i marchionimi che compaiono in supplementi locativi introdotti da $a$ sono formalmente più simili ai nomi comuni di quanto non lo siano quelli preceduti dalle preposizioni $d a$ e presso, senza articolo. Questo ha un influsso sulle scelte linguistiche delle imprese (cf. Janner 2012a: 165-168 per le differenze di autorappresentazione tra le aziende svizzere Migros e Coop). Carattere di nome proprio e prestigio, d'altra parte, possono essere coincidenti: si fanno acquisti alla Coop o da Coop, ma un abito firmato si comprerà da Prada, non certo alla Prada. La preposizione $d a$, peraltro, è quella che si combina con gli antroponimi nelle espressioni (mangiamo) da Gianni, (vado) da Lea (cf. La Fauci 2009: 208).

Varietà di realizzazioni formali del supplemento locativo si incontrano anche in relazione al predicato verbale lavorare:

(15) Quando lavoravo alla Fiat e vedevo che un lavoratore col figlio malato non poteva stare a casa ad assisterlo, leggevo quel fatto come un sopruso, una violazione agli insegnamenti di carità. (CORIS STAMPAPeriodici)

(16) “[...] Aveva tanto male, ma non ne parlava quasi. Come della fabbrica, prima. Quando lavorava alla Michelin mi diceva soltanto che faceva lavori brutti, che ogni tanto non riusciva a respirare. [...]”. (CORIS STAMPAQuotidiani)

(17) Era stato Enrico di Napoli Alliata, un uomo colto e illuminato, di grande capacità professionale, che allora lavorava all' $A G I P$, a trasmettermi la sua passione [...]. (CoLFIS, periodico)

(18) Pietro [...] ha 32 anni, è laureato in biologia e ha cominciato a lavorare in Ferrero nell'85, prima nello stabilimento di Allendorf, in Germania, e poi ad Alba [...]. (LaR)

(19) Ma negli anni Sessanta di questo secolo Vittorio Valletta ha chiamato i meridionali a lavorare in Fiat, perché erano indispensabili per tenere il ritmo dell'impetuoso sviluppo dell'automobile [...]. (CORIS STAMPAPeriodici)

(20) È un dialogo a distanza con tutti quelli che hanno denunciato la sua scalata ed è anche un decalogo del berlusconismo adattato all'azienda Mondadori: Non vogliamo prenderci rivincite con nessuno. Da domani davanti a tutti quelli che lavorano in Mondadori, ci sarà una pagina bianca. (LaR) 
Nel $\$ 3.1 .2$ si è osservato che i costrutti entrare in e entrare alla presentano una differenza di valore, dove entrare in può avere interpretazione finanziaria; l'alternanza tra lavorare alla e lavorare in, al contrario, parrebbe piuttosto di timbro stilistico e diacronico, il secondo tipo essendo più recente (cf. La Fauci 2009: 209) e parte ormai dell'italiano tendenziale. Stefano Bartezzaghi (2010: 89-90) registra la costruzione "in + nome dell'azienda per cui si lavora" come uno dei "tormentoni" dell'italiano contemporaneo e osserva: "La misteriosa trasformazione si è prodotta negli anni della ristrutturazione industriale e finanziaria. Per qualche ragione i posti di lavoro hanno perso il diritto agli articoli determinativi." Nei contesti delineati l'utilizzo della preposizione in per i nessi a valore locativo è un tratto in espansione (cf. Zardo 1997: 39n) e non si limita più esclusivamente a chi ha una relazione di lavoro con l'azienda, al contrario di quanto notava in passato Renzi (1988: 396).

Il nesso in Nmc si diffonde peraltro in molteplici contesti sintattici:

(21) Ecco Maura Monteverdi, in Barilla da ventiquattro anni, e Andrea Repetati, da uno soltanto. Tutti e due ripetono: "Pietro Barilla? Uno che quando ti incontrava ti salutava per primo". (LaR)

(22) A 48 anni Cravero entra nel gotha del gruppo. Complice l'improvviso abbandono del suo capo, uno dei principali collaboratori di Luca De Meo diventa così il numero uno in Alfa Romeo. (CORIS MON2008_10)

(23) È dello stesso parere Davide Grasso, laurea in economia e commercio a Torino, assistente di marketing in Gft e poi account executive all'Armando Testa e dopo il master alla Sda Bocconi una carriera di successo in Nike [...]. (CORIS STAMPASupplementi)

(24) Sulla stessa lunghezza d'onda lo scrittore Corrado Stajano, per molti anni consulente dell'Einaudi, una breve esperienza in Mondadori: I sistemi di organizzazione industriale saranno applicati all'editoria con una rigidezza maggiore che nel passato. (LaR)

(25) I tre sindaci - il presidente Franco Iorio e i due membri del collegio Pierluigi Martinelli e Aldo Migliorisi - sono arrivati in Mondadori alle 15. (LaR) ${ }^{46}$

46 Questo è l'unico esempio in cui al marchionimo sia associata l'interpretazione di 'luogo fisico'. 
Il costrutto lavorare per presenta una diversa sfumatura di significato:

(26) In quest'occasione è stato allestito uno spazio in omaggio a Wifredo Ricart, geniale ingegnere spagnolo che ebbe occasione di lavorare per l'Alfa Romeo e che ideò e costruì le celebri vetture spagnole Pegaso. (CORIS STAMPASupplementi)

(27) In due settimane Young ha visitato una dozzina di stabilimenti che lavorano per Nike in Cina, Indonesia e Vietnam parlando con il management e i lavoratori [...]. (CORIS STAMPASupplementi)

La preposizione per non è sostituibile con in o alla, che porterebbero all'interpretazione "essere impiegato presso", che invece non vale per questi costrutti.

Seguono altri esempi di supplementi locativi con indicazione del luogo di lavoro:

(28) Silvio, forse recitando un copione studiato a lungo con la moglie, ha riferito della sua vita piena di guai cominciati due anni e mezzo fa quando, assunto all'Alfa, era stato messo poco dopo in cassa integrazione e quindi licenziato. (CoLFIS, quotidiano)

(29) Un lavoro ce l'ha già Enrico Tauraso, 20 anni, di Fossano: Sono operaio alla Michelin. A dicembre comincerò il servizio civile. (CORIS STAMPAQuotidiani)

(30) Di Bartolomei a Roma aveva sposato Marisa, un'affascinante ex indossatrice, divorziata e madre di un figlio, che faceva la hostess all'Alitalia. (CoLFIS, quotidiano)

(31) "Spero che non vengano rifatti gli errori del passato", ha ammonito il novello Mosè, Roberto Danieli. Errori che i tre fratelli trevigiani - con Roberto in Diadora lavorano i fratelli Diego e Luigi - hanno saputo evitare abbandonando negli anni 60 il loro settore di punta, gli scarponi da sci. (LaR)

(32) Dietro all'Ethical Coffee Company c'è la figura di Jean-Paul Gaillard, fino al 1997 impiegato presso Nestlé, dove ha contribuito al successo delle capsule Nespresso. (SM 5/10)

Si osserva di nuovo che l'unico caso di presso ricorre nei dati di Spendere Meglio. 
I nessi "passaggio + supplemento locativo" hanno interpretazioni diverse a seconda dei contesti d'uso:

(33) Dani comunque viaggia veloce, esattamente come viaggiano veloci i rumors su un suo possibile passaggio in Yamaha nel 2010. (CORIS MON2008_10)

(34) Il passaggio [di Andrea Aghini] alla Lancia arriva alla fine del 1991: "Mi hanno messo alla prova nei rally di Sanremo e Spagna, poi mi hanno ingaggiato per alcune gare del '92 [...]". (CoLFIS, quotidiano)

(35) L'eventuale ingresso degli Agnelli, nel quadro di un passaggio della Fiat Auto alla Renault, complicherebbe ulteriormente le cose e la banca Lazard sarebbe stata incaricata di trovare una soluzione tecnica a questo problema. (CoLFIS, quotidiano)

Nei primi due esempi è questione del trasferimento di un pilota da una squadra a un'altra. In (35), invece, il soggetto non è un individuo, bensì la Fiat Auto: si tratta qui dell'inclusione di un'impresa in un'altra.

Infine va ricordato che anche marchionimi interpretabili come prodotti sono passibili di ricorrere in supplementi locativi:

(36) Le etichette non riportano la quantità [di anidride carbonica] aggiunta durante l'imbottigliamento, ma solo quella presente naturalmente alla sorgente $(2.150$ milligrammi per litro nella Ferrarelle, che è effervescente naturale, e appena 5,3 $\mathrm{mg}$ per litro per la Sanpellegrino che ha le bollicine aggiunte). (CORIS STAMPAPeriodici)

La presenza dell'articolo determinativo differenzia quest'uso da quelli visti sinora e lo allinea con quello dei nomi comuni (cf. anche nelle nostre Coop, es. 14).

\subsubsection{Mezzi di trasporto}

I supplementi predicativi che indicano l'utilizzo di un mezzo di trasporto si realizzano nei testi attraverso strutture formalmente diverse, interpretabili - secondo le tradizionali partizioni della grammatica come complementi di mezzo o strumento. 
Ricorre in particolare la struttura in Nmc:

(1) Qui sono meno popolare, posso girare liberamente per la strada senza essere riconosciuto da nessuno [...]. Giorni fa ho viaggiato in Harley Davidson con mia moglie Corinna da Las Vegas al Colorado [...]. (CORIS MON2001_04)

(2) Mi fidanzai con il primo che si presentò in vespa: era stupendo, tutte le mie amiche si erano invaghite di lui. (CoLFIS, periodico)

(3) Gli autogrill arrivarono negli anni 50, nell'Italia degli osti stupirono con la loro architettura futuribile, al popolo stanco delle fatiche in treno e di gite in lambretta offrirono un paesaggio nuovo e la tentazione peccaminosa del consumo. (CORIS STAMPAQuotidiani)

(4) Il caffè è un porto di mare per venditori ambulanti e spiantati, è una casbah dove transitano acrobati, avventurieri in moto Guzzi, crocchi di infelici dirimpetto a camarille di pomposi lestofanti. (LaR) ${ }^{47}$

(5) E la settimana successiva in piazza Prealpi la condanna a morte tocca a Filippo Cilione, 40, misterioso disoccupato che viaggia in Lancia Thema. (CoLFIS, quotidiano)

Prevalgono gli usi con marchionimi riferiti a ciclomotori ma non mancano marche di automobili (5), sulla falsariga dei costrutti in auto, in treno, in moto. E la struttura, nella sua riconoscibilità, si presta a ricorrere anche in usi traslati:

(6) Alla fine del campionato, il suo avvocato [di Fabio Capello] fa il giro dei tribunali e in un attimo lui è ridotto come un barbone. Diciassette miliardi vanno al Barcellona «che andrebbe retrocesso», cinquantuno alla società Juve che «dovrebbe essere sciolta»; alla fine a Capello non rimangono neanche i soldi per fare una gita in kleenex a Ostia. (CORIS STAMPAQuotidiani)

L'assenza di determinatore distingue questa struttura in Nmc da altri usi, per es. locativi (nella Ferrarelle), e dai casi in cui la presenza dell'articolo non determina una variazione di significato (investire in/nella Nike).

47 Si rammenta che in Moto Guzzi anche il nome comune è parte del marchionimo, benché capiti - come qui - che sia riportato con iniziale minuscola. La ricorrenza della struttura citata parrebbe mostrare che anche i parlanti percepiscono l'appartenenza di moto al nome di marca. 
Un altro tipo di supplemento locativo è introdotto dalla preposizione su:

(7) Nel '46, quando non c'erano mezzi, lo portavo in giro sulla mia Guzzi. (LaR)

(8) Due nibbi in divisa da poliziotto su una Guzzi sfrecciano da via Chiaia a via S. Anna di Palazzo [...]. (LaR)

(9) Con gli amici-comparsa (amici veri, per carità, salvo che molti non si conoscono fra di loro) dapprincipio un po' spaesati, infine anche loro perfetti nella parte: [...] due architetti trentenni, i più teneri di tutti, arrivati su una Yamaha [...]. (LaR)

(10) Dietro Haslam sono arrivati altri tre piloti britannici: Cal Crutchlow, su Yamaha, che ha concluso in 1'341, Jonathan Rea, con la Honda del Team Ten Kate, e James Toseland, con la Yamaha ufficiale [...]. (CORIS MON2008_10)

(11) È un'abitudine salire sulla Mercedes con la targa tedesca, attraversare uno spicchio di Costa Azzurra e mezza Italia. (CoLFIS, quotidiano)

(12) E alle 2,20 è lo stesso Ciarrapico, con la bozza dell'accordo, a saltare su una Mercedes ed andare in via Rovani da Berlusconi: Firmiamo, firmiamo, prima vado e prima torno, sorride. (LaR)

Anche in questo caso marchionimi che alludono ad autovetture e a motociclette si alternano entro una struttura che, rispetto alla precedente, si presenta come più flessibile: ricorrono sia usi determinati (e modificati) che non determinati dei nomi commerciali. L'uso senza determinatore, nell'esempio (10) e in altri simili reperibili nei dati, ricorre perlopiù in relazione a gare sportive.

Con marchi di automobili ricorre pure la preposizione (articolata) con:

(13) Poi via di corsa con la Bmw della ballerina verso l'aeroporto. (CoLFIS, quotidiano)

Nei dati sono inoltre attestate locuzioni preposizionali quali al volante di o in sella a(Det)/a cavallo di(Det) Nmc, che introducono nomi commerciali riferiti, rispettivamente, ad automobili e a ciclomotori:

(14) Dunque, alla barriera di Milano Sud è stato fermato e identificato un automobilista che al volante di una Bmw M5 viaggiava a 205 orari a un chilometro dal casello. (CoLFIS, quotidiano) 
(15) Il francese Thierry Van Den Bosch, tornato in sella all'Aprilia (team PMR$\mathrm{H} 2 \mathrm{O}$ ) dopo aver corso la passata stagione con la TM, si è aggiudicato la prima prova stagionale di quest'anno degli Internazionali d'Italia di Supermoto. (CORIS MON2008_10)

(16) Si passava la notte sotto una tenda, alla Tosa, anche quando la Formula 1 miliardaria era un pianeta impensabile, gli dèi del rombo correvano su due ruote e i primi capitoli dell'epica di questo circuito si scrivevano a cavallo di Guzzi, Gilera, Ducati, MV. (LaR)

Nel paragrafo precedente si è discusso del costrutto lavorare in Nmc. Esso ricorre nell'esempio seguente, ma con un'interpretazione diversa:

(17) All'epoca c'erano pochi privilegiati che possedevano una supercar di Maranello - ci correvano la domenica o andavano semplicemente a fare un giro fuori porta - ma per il resto della settimana andavano a lavorare in Maserati, perché era più comoda, dava meno problemi ed, eventualmente, poteva ospitare un paio di amici. (LaR)

Diversa è qui la conformazione sintattica del costrutto (si tratta del nesso "andare a lavorare (in Nmc)") e diverso è, paradigmaticamente, il valore della preposizione: a differenza del costrutto lavorare in Fiat/ in Mondadori, qui essa non è sostituibile da alla ma, eventualmente, da su/con + articolo.

\subsubsection{Abbigliamento}

La rassegna dei supplementi predicativi si conclude con un altro tipo di nesso in $\mathrm{Nmc}$ :

(1) Rosie Huntington Whiteley seduce Londra in Burberry $(<$ www.sfilate.it $>$ [titolo] $)^{48}$

(2) Già negli anni 50 la rivista Life aveva pubblicato in copertina il presidente Eisenhower in Lacoste, ma le magliette sono diventate davvero globali solo nel 1963, quando il figlio Bernard, che tuttora siede a capo dell'impero di famiglia, prese il posto di René [...]. (LaR)

$48<$ www.sfilate.it/133154/rosie-huntington-whiteley-seduce-londra-in-burberry> (3.11.2014). 
(3) Cos'altro sapeva [di Adelaide]? Ah, sì, che s'era accompagnata con Federico Laterza, una belva in gore-tex che al nostro stava sulla punta da morire, e che aveva una sorella più grande sorprendentemente carina. (Brizzi 1994: 15)

(4) "La passione per le scarpe calzate dai boscaioli americani - ha detto al New York Times Daniela Marino, direttrice di un'agenzia turistica - è scattata dopo una foto degli inizi anni ottanta in cui si vedeva Giovanni Agnelli in Timberland". (CoLFIS, quotidiano)

(5) Il mercato è immenso, ed è il neo-mercato della scarpa sportiva, delle Timberland per chi non ha mai messo piede su una barca, delle Tod's come Luca Montezemolo portate con il blazer, dell'estate affrontata in Birkenstock, non belle sì, da asceta camminatore, ma sfacciatamente comode. È il mercato degli infiniti pomeriggi in Reebok anche quando non si va in palestra. (LaR)

Il costrutto è modellato sul tipo in camicia, in giacca e cravatta, in ciabatte (e anche in bianco, in lungo, utilizzati in relazione all'abbigliamento). Si tratta di una metonimia della marca per l'abito o la calzatura. L'es. (3), "una belva in gore-tex", presenta una metonimia più complessa: il marchio registrato di un tessuto per gli abiti prodotti con esso.

Funzionalmente il nesso è una modificazione predicativa ${ }^{49}$ che manifesta diverse proprietà sintattiche: a seconda delle ricorrenze, ha una funzione comparabile al complemento predicativo del soggetto (1) o dell'oggetto (2; 4), alla modificazione aggettivale (3; 5: "infiniti pomeriggi in Reebok") o avverbiale (5: "l'estate affrontata in Birkenstock").

La principale caratteristica di questo nesso in Nmc è il suo ricorrere sempre senza determinatori, come nel caso dei veicoli (§3.4.2). La struttura pare inoltre riservata al nome commerciale non modificato: nel caso di nessi più complessi, come quello riportato di seguito, ricorre la preposizione con e non si dà sostituibilità con in Nmc.

(6) Margherita è quella con le Superga mezze rotte e la maglia con le chiazze d'unto. (LaR)

49 Un complemento di modo o maniera, in una tradizionale prospettiva semantica: cf. Zingarelli s.v. in $(1)^{6}$. 


\section{Conclusioni}

Il capitolo ha passato in rassegna alcune ricorrenze, accomunabili secondo un'ottica formale ma funzionalmente e combinatoriamente diverse, di nomi commerciali preceduti da preposizione. Si sono approfonditi nessi nominali in funzione argomentale, legittimati dal predicato; complementi del verbo e del nome, che fungono da modificatori rispetto a questi; supplementi predicativi (locativi e non).

L'accento è posto ancora una volta sulle relazioni e differenze sintattiche; pertanto si sono distinti i nessi preposizionali in base alla funzione sintattica che svolgono nella proposizione..$^{50}$ In costrutti quali "Young è stato assunto dalla Nike" e "non si può chiedere ad Alitalia di salvare Malpensa" il nesso introdotto dalla preposizione è legittimato dal predicato verbale a ricorrere nella proposizione e vi svolge funzione argomentale, di soggetto nozionale (in costrutti passivi) o di oggetto indiretto. Così pure nel caso dei complementi preposizionali del predicato, per es. "34mila persone vivono di Kodak" o "era più conveniente investire in Marelli", si può riconoscere al marchionimo funzione di argomento introdotto da preposizione (o, meglio, si tratta di predicati complessi: vivere di, investire in).

Nei casi restanti, per contro, la funzione di Prep Nmc è di tipo predicativo: il nesso modifica un nome ("la decisione della Fiat", "i camion dell'Iveco", "presidente di Adidas", "febbre da Playstation") oppure si pone come supplemento predicativo rispetto alla proposizione semplice, nel caso dei nessi a interpretazione locativa ("8 [prodotti] costavano meno da Migros"; "sono operaio alla Michelin"). Infine, i costrutti del tipo in Vespa e in Birkenstock sono utilizzati tanto come modificazioni di un verbo ("ho viaggiato in Harley Davidson", "saltare su una Mercedes") quanto come modificazioni di un nome ("gite in lambretta") oppure ancora come complementi predicativi di un nesso argomentale ("si vedeva Giovanni Agnelli in Timberland").

50 Tradizionalmente i complementi, "considerati tutti a pari titolo elementi facoltativi della frase", sono suddivisi secondo criteri semantici e interpretativi, ciò che - come osserva Lo Duca (2010) - rappresenta il principale difetto dell'analisi della frase. 
I principali aspetti combinatorî considerati sono due: la preposizione (tipologia; presenza/assenza) e la determinazione (presenza/ assenza), in parte correlate.

Mentre per la maggior parte delle strutture non si riscontrano corrispondenze con costrutti ove ricorra una preposizione diversa, per un'ampia fetta dei supplementi locativi si presenta una sostanziale corrispondenza tra i nessi alla Nmc, da Nmc e presso Nmc. Tali preposizioni, in particolare le più frequenti $a$ e $d a$, possono diventare un dispositivo stilistico per mettere in luce faccette diverse del nome commerciale: negozio (aspetto di nome comune: alla Coop come al supermercato) e persona giuridica (aspetto antroponimico: da Coop come da Gianni o dal fornaio).

La corrispondenza tra costrutti con e senza preposizione concerne perlopiù $d i$, in particolare alcuni costrutti $N d i N m c$ in corrispondenza funzionale con $N N m c:$ 1) quelli in cui è riconoscibile un rapporto tra prodotto e produttore, per es. "orologio (della) Swatch"; 2) quelli con un nome [+ umano] designante relazioni sociali o professioni svolte in seno all'azienda, come "operaio (dell')Alfa" o "presidente (della) Coop"; 3) quelli in cui il marchionimo ha funzione di aggettivo relazionale: "obiettivo (della) Fiat", "stand (della) Nutella". Questa variazione, che generalmente non si presenta con i nomi propri prototipici, costituisce uno dei tratti peculiari dei nomi commerciali a livello combinatorio..$^{51}$

L'ultimo aspetto ricordato, l'alternanza libera tra presenza e assenza di determinatori, è già stato osservato come caratteristico dei nomi commerciali (cf. §1.1.2). In relazione al ricorrere di preposizioni se ne possono delineare alcune specificità.

Con la preposizione $d i$ in genere l'articolo costituisce variante libera. Questa è, del resto, una caratteristica dei marchionimi che li differenzia dagli antroponimi: la giacca di Ivo e la giacca dell'Ivo sono sì equivalenti ma con una differenza di natura diatopica che non intercorre invece tra la decisione di Fiat e la decisione della Fiat. Costituiscono

$51 \quad$ Il fatto che nei costrutti senza preposizione non sia possibile integrare l'articolo davanti al nome commerciale ('orologio la Swatch), fatti salvi ovviamente i casi in cui un articolo sia parte integrante del marchionimo (orologio "La Strada" di Chopard), mostra inoltre che esso non vi svolge funzione di nome (ma di aggettivo). 
eccezione alla variazione libera i costrutti $N d i N m c$ in cui si riconosce una relazione contenente-contenuto (tazzina di Lavazza, pacchetti di Marlboro): qui il marchionimo non è determinato. Vi è inoltre il caso dei marchionimi a carattere fortemente antroponimico, come Valentino o Calvin Klein, per i quali la versione con determinatore è meno probabile.

Quanto alla preposizione $a$, vanno distinti gli usi locativi, anche metaforici (entrare alla $N m c$ 'essere assunto presso $N m c^{\prime}$ '), dagli usi in funzione argomentale (per es. come introduttore di oggetto indiretto): i primi sono di norma articolati, mentre i secondi presentano l'alternanza libera ricordata altrove come tipica dei marchionimi.

La preposizione $d a$ può essere o non essere articolata quando introduce il soggetto nozionale di un costrutto passivo, mentre ricorre senza determinazione in strutture come sorriso da Colgate e quando ha funzione di supplemento locativo (in vendita da Ikea), il che ne sottolinea il carattere di nome proprio rispetto alla variante con preposizione $a$, usualmente articolata.

Con la preposizione in si delineano casi diversi. I tipi in Fiat, in Vespa, in Lacoste (come supplementi predicativi) non sono articolati. Gli usi locativi (nelle nostre Coop) sono perlopiù articolati. Non ricorre un determinatore, per contro, qualora al costrutto in Nmc corrisponda il tipo a(Det) Nmc: passaggio in Yamaha/passaggio alla Lancia, lavorare in Fiat/lavorare alla Fiat. Altri supplementi che modificano il predicato verbale ricorrono sia con, sia senza determinatore, laddove in alcuni casi si delinea una differenza semantica tra le due varianti (entrare in $N m c$, detto di persone che vengono assunte da un'impresa; entrare nella $N m c$, detto di enti che partecipano finanziariamente alle sorti di una ditta), mentre in altri la variazione non ha conseguenze sul significato (investire in/nella Nmc).

L'analisi dei nessi preposizionali condotta secondo una prospettiva sintattica e funzionale, qual è quella proposta da La Fauci (2009), si è rivelata più fruttuosa, per la comprensione e la categorizzazione di tali nessi, di quanto non sarebbe un'analisi tradizionale dei complementi, fondata su interpretazioni del valore semantico (si è già visto quanto questo aspetto sia spesso difficile da valutare in rapporto ai marchionimi). 
Emergono, anche nel caso dei nessi preposizionali, alcune specificità dei nomi commerciali in una prospettiva sintattica, soprattutto per quanto riguarda la determinazione, correlata non solo alla presenza di determinate preposizioni ma anche alle caratteristiche sintattiche dei costrutti ove ricorrono i marchionimi. Combinati in unità maggiori, al livello della proposizione, essi presentano altre strutture e caratteristiche tipiche: è quanto si approfondirà nel capitolo che segue. 


\section{Sintassi della proposizione}

\section{Introduzione}

Sin qui l'analisi contestuale dei nomi commerciali si è focalizzata su un livello microsintattico, prendendo di mira le combinazioni all'interno di nessi nominali e preposizionali. Funzionalmente, si è visto, $\mathrm{i}$ marchionimi si presentano come predicazioni, eventualmente nominalizzate. Ma che cosa avviene quando entrano nella visuale predicazioni (verbali), quando insomma dal nesso nominale si passa a considerare la proposizione?

Questo capitolo approfondisce in che modo i marchionimi entrano a far parte di un complesso frasale, quali sono le funzioni che vi manifestano (soggetti, oggetti, predicati) e quali le modalità con cui si combinano con i verbi. L'obiettivo è approfondire le relazioni che $\mathrm{i}$ nomi manifestano entro la proposizione, tanto con altri elementi, quanto con l'insieme del nesso (cf. La Fauci 2009: 35).

Quanto alle funzioni rivestite entro la proposizione, non stupisce trovare nomi di marca in funzione di soggetto, di oggetto diretto e indiretto, di predicato nominale nel caso di costrutti copulari. In questi casi i marchionimi - articolati o non articolati, secondo le modalità già osservate a proposito del nesso nominale - non dimostrano particolarità combinatorie rispetto ad altri nomi, siano essi propri o comuni. In questo senso, anche sulla base delle osservazioni compiute nei capitoli precedenti, è lecito ipotizzare che la maggiore varietà e specificità morfologico-sintattica dei nomi commerciali si riscontri a livello di nesso e non di proposizione. ${ }^{1}$

1 Si veda al proposito quanto osserva Kolde (1995: 407) in merito alla sintassi proposizionale dei nomi propri: "Je höher man in der Hierarchie der Grammatik auf der Suche nach Spezifika der EN steigt, um so seltener wird man fündig”. Lo studioso si domanda se ciò sia da ricondurre a lacune nel sapere dei manuali oppure al fatto che, a livello di proposizione, i nomi propri presentano poche 
Per i marchionimi nella frase, dunque, non si osserva niente di speciale? Uno sguardo ai costrutti più frequenti nei dati porta a supporre che non sia così. È infatti possibile evidenziare alcune specificità dei nomi commerciali a livello di proposizione, che riguardano aspetti sia latamente semantici, sia strutturali e sintattici.

In primo luogo va approfondita la questione relativa alle restrizioni di selezione: ci sono predicati verbali che pongono condizioni restrittive sul soggetto che legittimano, in particolare correlate al tratto [ \pm umano]. Nel $\S 4.1$ si chiarirà cosa ciò comporta per i nomi commerciali che manifestano funzione di soggetto.

In secondo luogo, sulla scia delle osservazioni di Grass (2002) sui "verbi supporto", si studiano alcune combinazioni ricorrenti di predicato e oggetto diretto ( $\$ 4.2)$, che contribuiscono a delineare il significato specifico dei nomi commerciali coinvolti.

In terzo luogo si mettono in rilievo esempi di marchionimi in funzione di predicati nominali, combinati a essere e altri verbi copulari (§4.3). In questi casi la presenza o assenza di determinazione permette di distinguere tra due tipi, nominale ("l'auto dei killer era un'Alfa 33 nera") e aggettivale ("La ricetta è Findus"), quest'ultimo riconducibile alla funzione predicativa descritta nel cap. 2 .

Una volta esauriti tali aspetti, relativi alle funzioni grammaticali nucleari (cf. La Fauci 2009: 80), l'attenzione si sposta su tre tipi di strutture che spiccano per la frequenza relativamente alta con cui ricorrono nei dati.

La prima struttura contempla un nome commerciale che segue direttamente il predicato verbale: "volare Alitalia" (§4.4). Esso è riconducibile a un valore che qui si definisce avverbiale; caratteristiche combinatorie permettono, benché non in tutti i casi, di distinguerlo dalla funzione solo in apparenza simile di oggetto diretto.

Nella seconda struttura analizzata, "quel cowboy targato Marlboro" (§4.5), il nome commerciale segue un participio (targato, firmato, griffato); la struttura costituisce una predicazione nominale relativa a un altro nome. Il marchionimo può essere ricondotto funzionalmente al soggetto di un costrutto passivo ("Marlboro targa il cowboy"); in senso

differenze sintattiche rispetto ai nomi comuni. Sulla base dei dati analizzati si propende qui, in linea di massima, per la seconda spiegazione. 
metalinguistico, è interpretabile come attributo nominale mediato e introdotto dal participio, il quale a volte non è necessario alla compiutezza del nesso.

Un terzo tipo di struttura, "alla Renault volevano Prost" (§4.6), è costituito da alla $N m c$ + predicato verbale alla terza persona plurale, senza soggetto manifesto. Esso esemplifica una specificità dei nomi commerciali, che sono al contempo persone giuridiche e luoghi. La struttura trova un equivalente funzionale nel costrutto in cui il nome commerciale con valore metonimico funge da soggetto del predicato ('la Renault voleva Prost').

\subsection{Funzione di soggetto e restrizioni di selezione}

Vi sono nomi commerciali che ricorrono come soggetti in combinazione con predicati che legittimano un contesto ristretto per il soggetto (cf. La Fauci 2009: 62), in particolare relativi alla sfera della comunicazione (i cosiddetti verba dicendi):

(1) Cosa dice Opel al riguardo? (SM 4/07)

(2) La Renault ha già fatto sapere che non accetterà marcia indietro sulla tecnologia delle sospensioni attive e sull'elettronica. (CoLFIS, quotidiano)

(3) Come avevamo annunciato ieri l'Alfa Romeo ha comunicato ufficialmente il suo ritiro dalla formula uno. (LaR)

(4) In merito al suo olio spagnolo, Coop scrive: «La classificazione "lampante" si basa su un singolo panel di degustazione, pertanto non la riteniamo ammissibile. [...]». (SM 4/10)

(5) Cablecom dichiara che la maggior parte dei clienti internet conosce già le nuove offerte. (SM 5/10)

(6) Prima la Nissan ci suggeriva di alzare il livello del carburatore, ma non serviva a niente, poi di abbassarlo ma anche questo non risolveva. (CoLFIS, periodico) 
(7) Le reazioni delle due case non si sono fatte attendere: la Fiat, come al solito, $h a$ smentito, Renault si è trincerata dietro un "no comment". (CoLFIS, quotidiano)

I nomi commerciali di questi brani manifestano un tratto [+ umano] in correlazione ai predicati verbali che li legittimano come soggetti (dire, comunicare, dichiarare ecc.) e sono pertanto interpretabili come metonimie: il marchionimo sta per il portavoce della ditta. ${ }^{2}$

Anche la struttura esemplificata di seguito presenta sia il tratto [+ umano] del nome, sia la sua natura metonimica, benché esso non vi compaia come soggetto:

(8) Secondo la Volkswagen, queste vetture manifestano un effetto "fogging" sul parabrezza più che dimezzato rispetto a quanto avveniva in passato. (CoLFIS, periodico)

(9) Secondo Migros non vi sono rischi per i consumatori. (SM 6/05)

(10) Secondo Lavazza, che ha il $44 \%$ del mercato italiano, il costo aumenterà da fine aprile. $(\mathrm{LaR})^{3}$

Sono brani in cui il nome commerciale è soggetto alle restrizioni che si riconoscono per i soggetti di verba dicendi, ossia si presenta come [+ umano]. Lo stesso vale per la struttura "Alla Fiat dicono...", che sarà analizzata in seguito ( $\$ 4.6 .1)$.

Altri predicati verbali pongono condizioni restrittive sul soggetto:

(11) I nostri parlamentari sanno anche molto bene che la Fiat ha deciso di vuotare il sacco e la Fiat ha in Italia e all'estero millecento aziende. (CoLFIS, quotidiano)

(12) La PepsiCo si sta dando da fare per cambiare la sua immagine di nemica dei carciofi. (CoLFIS, periodico)

2 Il costrutto appare anche al passivo: "È morto sabato scorso a Milano, all'età di 92 anni, Giovanbattista Guidotti, capo collaudatore e pilota dell'Alfa Romeo per un cinquantennio. La notizia è stata comunicata dalla stessa Alfa Romeo" (CoLFIS, quotidiano).

3 Cf. inoltre: "Le aziende hanno logiche strane. Secondo loro, intendo quelli della Lavazza, c'era una sorta di cannibalizzazione del prodotto: in pratica lo spot era diventato più famoso del caffe" (LaR). 
(13) Poi, nel maggio '92, un secondo passo: la Barilla compra per 80,4 miliardi un altro $10 \%$ della Pavesi, va in maggioranza, la privatizzazione è compiuta. (CoLFIS, quotidiano)

(14) Per portare a termine l'operazione mi sono dovuto rivolgere agli sponsor, perché noi non eravamo in grado di affrontare tutte le spese. La Fuji Film, il settimanale "Il giornalino" e l'Algida ci hanno dato quindi volentieri una mano. (CoLFIS, quotidiano)

(15) L'Alfa piange Guidotti vinse a fianco di Nuvolari (CoLFIS, quotidiano [titolo])

(16) [...] da tempo Auchan è interessata ad un salto di qualità della presenza sul mercato italiano e, fin dall'inizio, ha seguito con attenzione le vicende del gruppo Standa. (CoLFIS, quotidiano)

Anche per questi contesti si riconosce un uso metonimico del nome commerciale. $^{4}$

La restrizione di selezione operata dal predicato verbale fa sì che ricorrano in funzione di soggetto nomi potenzialmente adatti a manifestare il tratto [+ umano] senza che ciò dia luogo a costrutti dotati di marcatezza interpretativa. Non tutti i nomi commerciali lo sono in ugual misura. Entro i dati analizzati, provenienti per larga parte - lo si ricorda - da testi giornalistici, vi sono nomi commerciali che ricorrono come soggetti in contesti ristretti e altri che non vi ricorrono, per es. Vespa, Post-it, Coccoina. Naturalmente costrutti quali "La Vespa dichiara che..." oppure "I Post-it decidono di..." non sarebbero impensabili ma sono meno probabili nei contesti enunciativi dei quali ci si occupa. A proposito di questi contesti è pertanto possibile tracciare un discrimine tra due tipologie di nomi commerciali: quelli che sono passibili di ricorrere come soggetti in contesti ristretti e quelli che non lo sono (o lo sono ma in maniera marcata, in contesti informativi). Per i primi si è parlato di rapporto metonimico, mentre per i secondi non è da escludere una dimensione metaforica quando si combinano a predicati che legittimano soggetti umani.

$4 \quad$ Vi sono inoltre numerosi casi di costrutti con passivi ridotti, che fungono da modificatori di nessi nominali: "Eccola qui l'auto elettrica «made in Ferruzzi» presto prodotta dalla Edison" (CoLFIS, quotidiano); "Marone aveva parlato tra l'altro delle tangenti pagate dalla Ferrarelle" (LaR). 
Soltanto i nomi cosiddetti d'azienda manifestano il tratto [+ umano]? Così parrebbe nella trattazione di Grass (2002: 231), che nomina solamente nomi d'impresa quando considera i nomi commerciali soggetto di verbi "réservés aux humains". Nondimeno, nei casi seguenti anche nomi con il valore di 'autovettura' presentano tratti di agentività:

(17) Nel cortile attendeva una Bmw nera debitamente infiorata. (CoLFIS, quotidiano)

(18) All'improvviso, una Land Rover blu si era messa in coda alla loro auto. (CoLFIS, quotidiano)

(19) La sua opera di convinzione fu tale che una Peugeot 504 grigia con una bandierina tricolore si fermò un'ora dopo all'ingresso dello slum di Anand Nagar. (CoLFIS, libro - D. Lapierre, La città della gioia)

(20) L'incidente, che pare causato dalla Bmw dei Tedeschi, è accaduto mentre nella zona infuriava un violentissimo temporale. (CoLFIS, quotidiano)

(21) Presenti sul mercato da ormai più di un decennio, le Lancia Delta delle prime serie lamentano con una certa frequenza l'insorgere di ruggine a livello di sottoporta, portellone e parafanghi. (CoLFIS, periodico)

Si consideri inoltre il seguente esempio:

(22) La Fiat ha perso la battaglia a difesa di quota 6.000 ed è planata, senza segni di reazione verso 5.950 lire. (CoLFIS, quotidiano)

Qui il tratto [+ umano] attribuibile a La Fiat (in riferimento alle azioni dell'azienda omonima) gli deriva dalla combinazione con il predicato "perdere la battaglia"; in questo caso ci si muove più sulla direttrice metaforica che su quella metonimica, come avviene invece nei casi precedenti. Accade infine che la relazione con l'oggetto diretto permetta di specificare, correlativamente, il valore del nome commerciale soggetto:

(23) Barilla e Bertolli hanno ritirato dal mercato tonnellate di sughi pronti. (SM $5 / 03)$

La relazione (definibile come possessiva) che lega Barilla e Bertolli, soggetti [+ umani], all'oggetto diretto è del tipo "produttore-prodotto": 
i sughi pronti sono di Barilla e Bertolli; le due imprese designate dai marchionimi non potrebbero ritirare dal mercato i sughi pronti di Coop.

In tutti i contesti visti nel presente paragrafo, si può affermare in conclusione, il rapporto del nome commerciale in funzione di soggetto con il predicato verbale (e con altri elementi della proposizione) contribuisce a delineare il valore del nome proprio.

\subsection{Oggetti diretti in combinazione ricorrente con un predicato}

Questioni di legittimazione si riscontrano non soltanto relativamente ai soggetti ma anche con gli oggetti diretti. Degli ipotetici "bere una Mercedes" o "guidare l'aspirina" sono indubbiamente più marcati rispetto ai nessi che compaiono negli esempi seguenti:

(1) Immagino che se sul torace di un terzino ci fosse tatuato "Leggete la Repubblica" oppure "Bevete Ferrarelle" qualcuno sarebbe già intervenuto. (LaR)

(2) [Il monsignore tradizionalista] non beve Coca-Cola, né mangia hamburger e pop corn [...]. (CoLFIS, quotidiano)

(3) [...] chi guida una Mercedes sa invece di avere in mano la vettura che si svaluta di meno [...]. (CoLFIS, periodico)

Ecco altre combinazioni ricorrenti di predicato verbale e marchionimo come oggetto diretto:

(4) Il Meloni aveva parcheggiato la sua Mercedes nei pressi ed era salito a chiamare l'amico [...]. (CoLFIS, quotidiano)

(5) [...] ma lei non sa quanto corro, ho dei crampi tremendi, l'altro giorno ho mangiato due Baci Perugina e davvero non riuscivo più a fermarmi [...]. (LaR)

(6) Già da alcuni anni circolavano rapporti secondo cui una persona che ha sofferto un infarto può ridurre il pericolo di un secondo attacco prendendo regolarmente l'aspirina $[\ldots]$... (LaR) 
Grass (2002: 228) sostiene che i marchionimi relativi a un prodotto sono associati a una classe - per es. alimenti, bevande, abbigliamento - che seleziona un supporto verbale o "operatore" appropriato. Si avrà pertanto "ich fliege Lufthansa", dove a Lufthansa si può sostituire il nome di qualsiasi compagnia aerea; si avrà "ich trinke Warsteiner", con una marca di birra; ma non "*ich fliege Warsteiner" (Grass 2002: 226). Negli esempi di Grass i marchionimi non sono determinati; tale tipo di struttura, in cui il nome non funge (necessariamente) da oggetto diretto, sarà analizzato più avanti (§4.4). Per il momento basti osservare che le affermazioni dello studioso sono applicabili alle combinazioni di predicato verbale e oggetto diretto viste or ora.

Spesso i nomi commerciali in funzione di oggetto diretto appaiono dunque "specializzati" rispetto a uno o più predicati verbali; se ne può parlare in termini di una probabile collocazione. In questo senso non vi si applica quanto osserva Harris (1952: 6), nell'ambito della linguistica distribuzionale, a proposito dei nomi personali, che possono avere la stessa distribuzione: "for every sentence containing Bill we may find an otherwise identical sentence containing Jim instead". In costruzioni quali mangiare Nmc o guidare Nmc non si troveranno forzatamente gli stessi nomi commerciali.

Data la natura semantica e la definizione stessa dei nomi commerciali, sono frequenti i casi in cui il predicato verbale appartiene alla sfera del commercio:

(7) Senza ricetta, possiamo vendere solo aspirine, analgesici, antinfiammatori blandi [...]. (CoLFIS, quotidiano)

(8) I veneti, lo scorso anno, hanno speso ben 25 miliardi per acquistare Jacuzzi, divenuta evidentemente uno status symbol $[\ldots]$. (LaR)

(9) Tipi così bisogna metterli a posto come ha fatto l'Agnelli con il Carlo Pesenti che voleva sfidare la Fiat con la Lancia, quando il Pesenti è stato alla frutta ed è andato da Agnelli per vendergli la Lancia gli ha fatto fare un'ora di anticamera. (CoLFIS, libro - G. Bocca, Metropolis)

(10) Fino a quando la dieta mediterranea avrà tanti adepti, il consumo di olio di oliva rimarrà elevato: oggi un italiano ne utilizza in media 12 chili all'anno. Lo sanno bene i giganti dell'industria alimentare: Unilever ha rilevato i marchi Dante, Bertolli e S. Giorgio, Eridania ha acquistato Carapelli, Nestlé ha preso Sasso. (LaR) 
Questi predicati non si specializzano con una particolare categoria di marchionimi. Si presentano però due scenari diversi, esemplificati rispettivamente da (7-8) e da (9-10).

A un livello superficiale la differenza si pone tra nomi commerciali riferibili a prodotti (aspirine, Jacuzzi) e nomi commerciali riferibili a marchi o imprese (la Lancia, Carapelli). In termini di rapporti combinatorî si può invece affermare che nei costrutti del primo tipo il nome commerciale può comparire anche al plurale (nell'es. 7 è presente un plurale anche formale), mentre nel secondo tipo ricorrono nomi al singolare (femminile), eventualmente preceduti da articolo determinativo.

Si veda peraltro l'es. seguente:

(11) Umberto Agnelli [...] si inventa un nuovo "business": diventerà il primo grande polo alimentare italiano. [...] Le cose, all'inizio, vanno bene: grande alleanza con la Bsn, colosso francese guidato da un mago come Antoine Riboud e poi via ad una grande campagna di "shopping". Viene acquistata così la Ferrarelle, poi la pasta Agnesi, la Panzani e, infine, il grande colpo con la Galbani. (LaR)

I nomi commerciali che ricorrono come oggetti diretti (nozionali) di acquistare, nella proposizione passiva che chiude il brano, non sono solo al femminile singolare ('ditta', 'marca'): vi è pure, caso particolare, "la pasta Agnesi", in cui il marchionimo ha funzione predicativa rispetto a un nome comune relativo a un prodotto. Il contesto del brano non dà adito a un'eventuale ambiguità, per cui si potrebbe pensare che sia un individuo (per es. "Umberto Agnelli") il soggetto, non manifesto, dell'ultima proposizione e che i nomi commerciali valgano come riferimento a prodotti. In realtà dal contesto è chiaro che essi - anche "la pasta Agnesi" - alludono a marchi.

Nell'es. (10) si legge "Eridania ha acquistato Carapelli", in una struttura in cui sono legittimati a ricorrere come soggetti altri nomi commerciali:

(12) Pochi messaggi, chiarissimi, quelli di Romiti. "Non è vero che la Fiat compra la Ferruzzi, anzi noi disinvestiamo e incassiamo una somma cospicua". (LaR)

È chiaro che qui il valore dei marchionimi, per usare una categoria semantico-referenziale, è quello di azienda o di marchio, non di prodotto. Ancora una volta, però, è il contesto proposizionale a permettere 
di stabilire tale valore: esso emerge dalle combinazioni, non da considerazioni astratte di ordine referenziale (sulle quali cf. Parte I, §3.2).

\subsection{Costruzioni copulari}

Si approfondiscono ora alcuni esempi di nomi commerciali che ricorrono in costruzioni contenenti verbi copulari, rivestendo in alcuni casi funzione predicativa.

\subsubsection{Nomi commerciali e predicato d'identità}

(1) L'auto dei killer era un'Alfa 33 nera, risultata rubata. (CoLFIS, quotidiano)

(2) La prima a introdurre una svolta fu proprio la Ford, che a metà degli anni ' 80 inaugurò una politica commerciale più aggressiva, offrendo motorizzazioni diverse allo stesso prezzo. (CoLFIS, periodico)

Si distinguono, in primo luogo, i marchionimi che ricorrono come predicati nominali (1) da quelli che ricorrono in costrutti detti identificativi o identificazionali (cf. La Fauci 2009: 145; Panunzi 2011), come (2), in cui il verbo essere costituisce predicato d'identità tra due espressioni pienamente referenziali.

Tendenzialmente sono ascrivibili a costrutti identificativi la maggior parte dei casi in cui i nomi commerciali introdotti da essere sono preceduti da articolo determinativo:

(3) L'auto più rispettosa dell'ambiente è, nell'opinione di chi si è seduto al suo volante, l'Audi. (CoLFIS, periodico)

(4) Una delle prime aziende automobilistiche ad attivarsi sui problema è stata la Volkswagen [...]. (CoLFIS, periodico)

(5) se si aggiunge che la committente di quei lavori è $l a S E A$ che è la società aeroportuale e quindi gli enti pubblici le istituzioni della Lombardia implicate in questo brutto affare io credo che questa denuncia va fatta (LIP ME 6) 
(6) Ieri protagonista indiscussa della riunione è stata la Fiat, sia per escursione di prezzo sia per quantità di scambi. Sono stati trattati circa 15,7 milioni di titoli della casa automobilistica [...]. (CoLFIS, quotidiano)

I marchionimi non hanno qui funzione predicativa, bensì si riferiscono a un'entità individuale della quale è predicata l'uguaglianza con quanto precede il verbo essere.

In questo tipo di costrutti il marchionimo ricorre anche come soggetto:

(7) La Sony fu la prima a realizzare l'Hdtv: nel 1986, in collaborazione con l'Nhk (la tivù di Stato nipponica), presentò il sistema Muse con scansione a 1125 linee/60 quadri al secondo. (CoLFIS, periodico)

(8) Ford Escort 1.616V Boston è l'unica vettura del gruppo a essere dotata di motore con distribuzione bialbero a quattro valvole per cilindro. (CoLFIS, periodico)

(9) Davanti Chili, Biaggi e Reggiani hanno dimostrato che l'Aprilia è la moto da battere, e Romboni col quarto tempo è stato il primo "giapponese". (CoLFIS, quotidiano)

Si delinea inoltre una sostanziale reversibilità del costrutto: "La prima a realizzare l'Hdtv fu la Sony" (cf. 7); "L'Audi è l'auto più rispettosa dell'ambiente" (cf. 3). Non vi è reversibilità, invece, nei casi in cui il nome commerciale soggetto si combina a essere più nesso nominale indeterminativo: "la Renault è una società pubblica nella quale la Volvo detiene il 20 per cento delle azioni" (CoLFIS, quotidiano).

Ricorre a volte, in entrambe le varianti del costrutto identificativo ( $X \grave{e} \mathrm{Nmc}$ e $N m c$ è X), un nome comune co-referente con il nome commerciale: auto, azienda, vettura, moto. Vi è dunque una predicazione implicita che si somma a quella d'identità: affermando che "L'auto più rispettosa dell'ambiente è l'Audi" si afferma pure che l'Audi è un'auto, nel contesto specifico della proposizione. Ecco un altro caso in cui il contesto permette di recuperare un possibile significato del nome commerciale utilizzato argomentalmente. 


\subsubsection{Nomi commerciali in funzione predicativa}

Si riprenda ora il brano riportato in apertura del paragrafo precedente:

(1) L'auto dei killer era un'Alfa 33 nera, risultata rubata. (CoLFIS, quotidiano)

In questo caso il nome commerciale, preceduto da articolo indeterminativo, ha effettivamente funzione di predicato nominale. Il nesso nominale indica appartenenza a una classe (cf. Panunzi 2011), la classe delle "Alfa 33", tra le quali rientra anche il referente designato dal soggetto della proposizione, "L'auto dei killer".

Altrove i nessi nominali che seguono la copula non sono preceduti da determinatore:

(2) Le case tedesche [...] sono impareggiabili dal punto di vista della qualità dei prodotti, anche i più banali: le lavatrici migliori del mondo sono Aeg o Miele, gli acciai più resistenti Krupp o Thiessen [sic], le macchine più sfavillanti Mercedes o Bmw, gli pneumatici più resistenti Continental, i trenini elettrici più prestigiosi Marklin [sic] [...]. (LaR)

(3) Sapete cosa rende il cacao Ferrero così unico? Il fatto che sia Ferrero, naturalmente. (pubblicità Ferrero, LaR, novembre 2016)

(4) Guildford: la famosa antenna tecnologica servirà ancora a qualcosa adesso che la Ferrari è Fiat e ha dietro le spalle il Centro Ricerche di Orbassano? (LaR)

(5) Secondo Del Buono, il Milan si deve far carico dell'impopolarità acquisita insieme al suo presidente, "perché c'è chi è contro questo governo e il Milan gli sta antipatico esattamente come tanta gente ha tifato contro la Juventus perché è Fiat. [...]" (LaR)

(6) MILANO - La Borsa è Fiat: così è stato ieri primo giorno del ciclo di ottobre, così sarà oggi se verranno confermati i prezzi caldi del pomeriggio. (LaR)

“La mia cucina non è McDonald's". PARLA VISSANI (LaR [occhiello, titolo])

Diversamente dagli esempi del §4.3.1, qui i nomi commerciali - in funzione predicativa - identificano non un individuo bensì una proprietà, che sarà, a seconda dei contesti, "essere di marca $N m c$ ", 5 "essere di

5 Così anche lo scambio di battute pubblicitario "Questo è svizzero!" - "No, Novi!". 
proprietà dell'azienda $N m c$ ", ma può avere pure contenuti predicativi diversi (esempi 6 e 7).

In quanto tale, naturalmente il predicato nominale non è reversibile: da La Borsa è Fiat non si avrà Fiat è la Borsa. Ciò permette di distinguerlo dagli esempi seguenti:

(8) Da una parte si sostiene che la Fiat, benché acciaccata, rimane ancora la miglior ragazza da marito che ci sia oggi in Europa. Anzi, se qualche produttore di automobili volesse sposarsi, la ragazza da scegliere è Fiat, in assoluto. (LaR)

(9) Da Parigi, la Fifa nega qualsiasi coinvolgimento. "Il nostro fornitore è Adidas, che tra l'altro non possiede fabbriche in Cina" precisa la Federazione. (CORIS STAMPAQuotidiani; virgolette aggiunte)

In (8) ricorre il nesso "è Fiat"; ma in questo caso si può avere anche "Fiat è la ragazza da scegliere", come pure, per (9), "Adidas è il nostro fornitore". Si tratta quindi di costrutti identificativi. Il fatto che i nomi commerciali non siano determinati non deve far pensare che si tratti di un uso predicativo (come nel caso di "Il fatto che [il cacao] sia Ferrero", 3 ), anche perché, sia in (8) che in (9), non vi sono differenze sostanziali rispetto al nome commerciale preceduto da la (cf. §1.1.2).

Riassumendo, i casi di nomi commerciali che seguono il verbo essere si possono distinguere nei seguenti tipi: 1) secondo elemento di un costrutto identificativo: uso nominale (ev. con determinatore in variazione libera con $\varnothing$ ); 2) predicato nominale: con determinatore, uso nominale (soggetto $=$ elemento di una classe); 3) predicato nominale: senza determinatore, uso aggettivale (esprime una proprietà del soggetto).

Nell'ultimo caso vi sono diverse possibilità di esplicitare la relazione tra predicato e soggetto: il classico slogan "La ricetta è Findus" può essere interpretato come "La ricetta dei prodotti è proprietà dell'azienda Findus", "La ricetta è di marca Findus" (è firmata Findus, si potrebbe dire; cf. infra, §4.5), o ancora "[Un prodotto] Findus è la ricetta (per cucinare bene)", con costrutto identificativo. La conformazione strutturale del predicato nominale consente di non specificare ulteriormente il tipo di rapporto con il soggetto lasciando libero corso a letture (sintattiche) diverse, come avviene non di rado con i messaggi pubblicitari. 


\subsection{Volare Alitalia. Usi avverbiali}

(1) Dal carnet di Versace, che tra l'altro veste Elton John, dovrebbero comunque uscire altre star. (LaR)

(2) Elton John veste Versace, e Versace firma anche l'allestimento: un'astronave di luci, una pedana rotante con, al suo centro, il cantante al pianoforte. (LaR)

Versace veste Elton John e Elton John veste Versace: i medesimi elementi danno luogo a due strutture sintatticamente diverse. La prima, infatti, è un costrutto transitivo, nel quale il nome proprio Elton John ha funzione di oggetto diretto, e ha un corrispondente passivo: "Elton John è vestito da Versace". Nel secondo caso, invece, non vi è una costruzione equivalente che abbia Versace come soggetto grammaticale: "Versace è vestito da Elton John". Si deve intendere che il nome commerciale funga qui non da oggetto diretto del costrutto ma - se si volesse cercare un'etichetta funzionale - da avverbio. È di questa struttura che si occupa questo paragrafo.

I costrutti come vestire Versace, lo si è argomentato nel §1.2.2, si contraddistinguono per l'assenza di alternanza libera tra marchionimi determinati e non-determinati: si pensi alla differenza che si presenterebbe con un ipotetico "Elton John veste il/un Versace". Anche qualora entrambe le forme ricorrano effettivamente, come nel caso di fumare Marlboro e fumare una Marlboro, la differenza che passa tra $\mathrm{i}$ due costrutti è diversa da quella che vige tra la Migros dice e Migros dice (con marchionimo soggetto). In quest'ultimo caso si è parlato di variazione libera, dal momento che la presenza del determinatore non influisce sul significato globale del costrutto; in relazione agli usi postverbali, invece, la determinazione manifesta valori correlativi diversi.

\subsubsection{Inquadramento diacronico}

La struttura, analizzata in vari studi sulla lingua pubblicitaria, presenta un predicato verbale (che eventualmente non legittima oggetto diretto; nella letteratura si parla spesso di "verbi intransitivi") seguito da un nome commerciale senza determinazioni o modificazioni, usato 
in maniera funzionalmente avverbiale (cf. Migliorini 1956: 78; Folena 1964: 119; Calabrese 1974: 172). A detta di molti, tale struttura rappresenta uno dei tratti caratteristici della "lingua della pubblicità": ${ }^{6}$ per Folena (1964: 118) si tratta di "[u]no dei casi più notevoli di spinta stilistica innovatrice esercitata attraverso lo slogan tanto nel campo della pubblicità commerciale quanto in quello della propaganda politica". Al contempo è un costrutto "saldamente radicato nel linguaggio comune", come scrive De Mauro (1967: 54), negando al linguaggio pubblicitario - che egli definisce "subalterno" - la carica innovativa che altri vi riconoscono. E continua tuttora a ricorrere in vari slogan (per es. "Chi vola Alitalia, noleggia Maggiore", del 2012), segno che non è ancora percepito come un costrutto usurato, benché circoli da vari decenni: si pensi a slogan storici quali "Corre giovane chi corre Agip", "camminate Pirelli" o al satirico e dissacrante "Scusate, abitualmente vesto Marzotto" aggiunto alla foto di Aldo Moro rapito dalle Brigate Rosse.

Come osserva Migliorini (1956: 78), la struttura è utilizzata in motti politici ("votate socialista", apparso dapprima in Francia nel 1936 e importato in Italia con le elezioni del 1946) e si diffonde al contempo nella lingua della pubblicità ("brindate Gancia", del 1936). Migliorini (1956: 77-78) lo definisce "un caso in cui la spinta esercitata dalla politica e dalla pubblicità ha fatto violenza alle norme grammaticali consuete, introducendo nell'uso linguistico un costrutto nuovo", ${ }^{8}$ benché non del tutto sconosciuto alla lingua francese o italiana: lo studioso lo paragona agli esistenti "parler français, parlare italiano", osservando inoltre la presenza di "un folto gruppo di aggettivi con valore di

6 Da lunga data: già nel 1964 Gianfranco Folena lo definisce un "tipo ora comunissimo" (Folena 1964: 118) e De Mauro ne scrive in un saggio del 1967 come di "un tipo già saldamente affermato" (De Mauro 1967: 54). Tra i primi, se non il primo, a occuparsi di tale struttura per l'italiano è stato Migliorini $(1952 ; 1956)$.

7 Si noti, qui come nello slogan di Alitalia e Maggiore, la reduplicazione di uno stilema, tratto tipico della funzione poetica della lingua (cf. Jakobson 1960) ripreso nella lingua pubblicitaria.

$8 \quad$ A proposito del quale Migliorini (1956: 78) commenta saviamente, non si sa se sarcastico o rassegnato: "Sgrammaticature? Da un punto di vista strettamente conservatore, sì. Ma è possibile immaginare una lingua immobile in un tempo in cui le due figlie dell'antica Fama, la Propaganda e la Pubblicità, levano clangori così alti da assordarci?". 
avverbi, parlar chiaro, risponder franco, giocar liscio ecc." (Migliorini 1956: 78). ${ }^{9}$

Il tipo pubblicitario "Brindate Gancia", insieme ai suoi emuli (Migliorini cita Viaggiate CIT e Camminate Pirelli, del 1948; Calabrese ricorda veste Facis e corre Agip), presenta "due novità: il verbo intransitivo e l'uso di un cognome con un valore quasi avverbiale" (Migliorini 1956: 78).

\subsubsection{Analisi funzionale: uso avverbiale o uso argomentale?}

(1) Insomma c'è da stare tranquilli se si vola Alitalia? Non tutti ne sono convinti e la fotografia dell'attuale situazione, pur così trasparente e rassicurante, non può rispondere a domande che solo adesso si affacciano nel mondo aeronautico. $(\mathrm{LaR})$

Il predicato verbale che ricorre in (1), volare, non è solito legittimare un oggetto diretto; un costrutto come "volare l'aereo" non sarebbe ben formato. È uno degli esempi di quella forzatura, di origine pubblicitaria, che ha ormai acquisito cittadinanza nella lingua comune.

Più frequenti sono i casi in cui il nome commerciale è introdotto da predicati verbali atti a legittimare un oggetto diretto:

(2) I ragazzi della Fiat [...] Indossano giubbotti di jeans, calzano Nike e portano lo zainetto "Invicta". (LaR)

(3) Da queste parti dell'Austria molta gente veste Benetton a giudicare dalla quantità di negozi che l'industria di Treviso ha disseminato in città e paesini. (LaR)

(4) Nei prossimi giorni i dirigenti gialloblù hanno in agenda un incontro per definire la sponsorizzazione con questo marchio: quella gialloblù sarà la prima squadra italiana di serie A a vestire Nike. (LaR)

(5) $[\ldots]$ con tutti questi punti Barilla, dicevo, e ne ho già la bellezza di 900, perché me li faccio dare anche da mia zia Ramperi Maria, che compera solo Barilla, e

9 Come osserva Calabrese (1974: 172), la pubblicità aveva già ripreso tali costrutti con aggettivi in funzione avverbiale: "Kadonett fissa morbido morbido", "bevete sano con Frizzina", "comprate sicuro, Marzotto". 
dalla mia vicina Iole Tancheri [...]. (CORIS NARRATRacconti - Aldo Nove, Woobinda) $)^{10}$

(6) Droga? No, fortunatamente Gianni, da quando era un bambino di 10-11 anni, fuma solo Marlboro. (CoLFIS, quotidiano)

E Don Giovanni e Leporello nel cimitero si fanno anche una buona sniffata di coca. Naturalmente si mangia McDonald: non fagiano innaffiato di Marzemino ma hamburger con ketchup e Coca-cola [...]. (LaR)

[...] le divertenti gag di Tullio Solenghi (che non fa rimpiangere i pur efficacissimi spot di Nino Manfredi) finito a bere Lavazza in un rilassante Paradiso. (LaR)

Folena (1964: 119) ricollega questa struttura alla giustapposizione di nome comune e "nome-etichetta" (calzature Superga, vestito Marzotto, sorriso Durban's), che "deve la sua fortuna allo stile commerciale" (Migliorini 1927: 341), ossia al tipo di struttura analizzata nel cap. 2 (in particolare §2.2.1). ${ }^{11}$ Per alcuni degli esempi proposti sopra si possono individuare combinazioni comparabili: vestire Nmc/abito Nmc, calzare

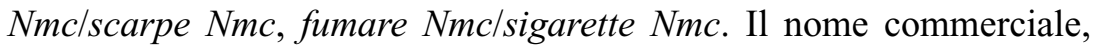
che in $N N m c$ ha funzione paragonabile a quella di un aggettivo, presenta funzione avverbiale in $V N m c$ e funge in entrambi i costrutti da "etichetta" morfologicamente invariabile, a valore predicativo e non argomentale.

Folena postula una differenza tra questa e la struttura transitiva:

anche coi verbi transitivi (per es. in calzate Superga) il sostantivo non è sentito come oggetto ma come determinativo avverbiale; quando si creò lo slogan di pubblicità collettiva calzate cuoio esso fu sentito come radicalmente diverso dall'altro bevete birra, cioè come l'equivalente grammaticale del costrutto camminate Pirelli al quale si contrapponeva (Folena 1964: 119).

10 V. anche "a scatola chiusa compro solo Arrigoni", di caroselliana memoria.

11 Folena (1964: 119) osserva che nella lingua pubblicitaria esistono "serie di costrutti paralleli nominali e verbali, dove il «cartellino» fa corpo col termine precedente e rimane invariabile: «Sorriso Durban's - Sorridete Durban's», «Scarpe Pirelli - Camminate Pirelli» ecc." Lo studioso non tiene conto del fatto che la relazione tra sorriso e Durban's è più complessa di quella tra scarpe e Pirelli, che è del tipo "produttore-prodotto" (cf. §2.2.1). Si può parlare di "calzature di/della Superga" (e si può affermare che "le Superga" sono "calzature"), ma non di "sorriso della Durban's"; tutt'al più di un "sorriso da/alla Durban's" (e "il Durban's" difficilmente si riferirà a un "sorriso"). 
Provando ad andare oltre il sentimento, si può esprimere tale differenza in termini combinatorî.

(9) Spot della Pepsi. Ce ne sono due [oranghi], in due gabbie, a uno viene data da bere Pepsi, a un altro Coca. (LaR)

In questo esempio i marchionimi Coca e Pepsi funzionano come nomi di massa. La non-definitezza equivale a un partitivo ("a uno viene data da bere della Pepsi, a un altro della Coca"), eventualmente anche a un articolo indeterminativo singolare: una Pepsi, una Coca. Nei brani riportati in precedenza, invece, l'inserimento di un articolo indeterminativo provocherebbe mutamenti significativi del costrutto, dando luogo, a volte, a costruzioni dall'interpretazione marcata ("compera solo una Barilla"?). Con (9) ci si trova dunque di fronte a un uso argomentale, non avverbiale, del nome commerciale.

Un'altra prova consiste nel fatto che il participio passato si accorda con Pepsi e Coca, al femminile. Ancora, l'esempio contiene un costrutto passivo, mentre negli altri casi è difficile ipotizzare un costrutto passivo equivalente ("'Benetton è vestita da molta gente"). Questo, più che l'assenza del determinatore, è un criterio dirimente nel decidere il valore argomentale o predicativo del nome commerciale.

Si prendano in considerazione i casi seguenti:

(10) Da allora sono passati non solo quattro anni, ma praticamente una rivoluzione nel settore alimentare. Nestlè ha comprato Buitoni, un nuovo gruppo, quello della Bsn-Ifil si è creato e ingrandito arrivando ad insidiare la Barilla nei settori della pasta e dei biscotti. (LaR)

(11) La Bianchi è stata acquistata dalla Piaggio: e la Bianchi-Piaggio ha comperato la Legnano. (LaR)

(12) Così per esempio Chloè, malgrado i suoi scarsi recenti successi, è stata acquistata dall'inglese Dunhill, rinomata fabbrica di pipe (LaR)

In questi costrutti, tanto attivi $(10,11)$ quanto passivi $(11,12)$, i marchionimi evidenziati sono identificabili univocamente come oggetti diretti anche se seguono il verbo comprare e non presentano determinazione o modificazioni, come in "mia zia [...] compera solo Barilla" (5). Il riferimento è qui al marchio o alla ditta, non ai prodotti. Inoltre l'aspetto perfettivo dei verbi di (9-12), in mancanza di specificazioni che lo rendano 
durativo ("ha sempre comprato Buitoni"), porta a interpretare l'azione come singolare, in opposizione a quanto si ritrova negli esempi (1-8), tutti al presente (o all'infinito).

\subsubsection{Interpretazione del costrutto}

Gli esempi riportati confermano quanto osserva Grass (2002: 227) in merito ai "verbi supporto" (cf. §4.2): anche negli usi avverbiali ogni nome commerciale si combina al predicato confacente alla classe di prodotti in cui rientra (oltre ai generici comprare o scegliere). Si hanno così vestire Adidas, bere Coca-Cola ecc.

D'altro canto, non per tutti i nomi commerciali in funzione avverbiale un predicato verbale specifico è immediatamente pensabile: a cosa combinare Goodyear, Barbie, Kodak? Qui entrano in gioco espressioni come sorridete Durban's, che si allontanano dal "verbo supporto" proponendo accostamenti creativi (spesso con intenti pubblicitari).

Il valore specifico del nome commerciale utilizzato avverbialmente non si può definire a priori ma discende dal contesto proposizionale e combinatorio.

(1) Pare che la Puma sia preoccupata a livello d'immagine dai guasti causati dalle non belle esibizioni di Becker. Anche perché in questo momento stanno salendo le quotazioni della tennista tedesca Stefi [sic] Graf, che guarda caso veste Adidas, diretto concorrente della Puma. (LaR)

(2) Nei momenti di riposo, però, le scarpe devono essere solo Diadora. La questione crea non pochi problemi. Molti giocatori sono infatti legati per contratto ad altre fabbriche: Altobelli calza Adidas, Conti è "proprietà" Puma, Bagni è pagato dalla Lotto. (LaR)

(3) Sono sensibili al problema [del lavoro minorile] anche i grandi calciatori: Ronaldo, Zidane, Baresi hanno girato spot per i palloni puliti. Michel Platini ha garantito che Francia '98 sarà un campionato del mondo non schiavista, si giocherà Adidas e il materiale verrà dal Marocco. (LaR)

Il marchionimo Adidas assume tre sfumature di significato diverse negli esempi (1-3), in combinazione con i predicati verbali vestire (abbigliamento), calzare (scarpe) e giocare (palloni da calcio e altro materiale 
sportivo). Anche in questo caso, come ricordato altrove per strutture diverse, non è generalmente possibile né necessario stabilire il referente preciso, con l'eccezione forse di marchionimi che identificano (anche) un prodotto, come (bere) Coca-Cola.

L'indeterminatezza non è solo semantica ma anche e in primo luogo morfosintattica. Si è già osservato che gli usi avverbiali non presentano determinatori, anche se gli stessi nomi, in altri contesti, compaiono preceduti da articolo. Proprio tale assenza di determinatezza gioca un ruolo cruciale nel delineare non tanto un'azione puntuale, come "bere una Pepsi" o "fumare una Camel", bensì una scelta abitudinaria e, in ultima istanza, uno stile di vita ("io fumo Camel" come "io voto liberale"; cf. i saggi di Migliorini sopra citati). Proprio qui lo stilema della lingua pubblicitaria identificato dagli studiosi trova la sua ragione d'essere e la sua forza perlocutiva.

Questo carattere dell'uso avverbiale si può osservare in due esempi riportati nel $\S 4.4 .2$ e qui ripresi:

(4) [I punti Barilla] me li faccio dare anche da mia zia Ramperi Maria, che compera solo Barilla [...]. (CORIS NARRATRacconti - Aldo Nove, Woobinda)

(5) Droga? No, fortunatamente Gianni, da quando era un bambino di 10-11 anni, fuma solo Marlboro. (CoLFIS, quotidiano)

In essi il nome commerciale è preceduto dall'avverbio solo e accompagnato, in (5), da un'indicazione di durata. Il costrutto avverbiale è dunque interpretabile nei termini di una dichiarazione relativa allo "stile di vita" (cf. §1.1.5 su "scegliere Alfa 159").

\subsection{Quel cowboy targato Marlboro. Etichette metalinguistiche}

\subsubsection{Aspetti predicativi della struttura}

(1) Ben 9 famosissime aziende [...] abbinano ai loro prodotti utilissimi oggetti da tavola firmati Guzzini. (CoLFIS, periodico) 
(2) Dopo il formaggio Bel Paese firmato Agnelli arrivano gli alberghi targati Fiat. $(\mathrm{LaR})$

(3) Sarà targato Olivetti il software delle Ferrovie italiane. (LaR)

La struttura oggetto di questo paragrafo presenta un participio passato immediatamente seguito da un nome commerciale; il complesso funge da modificatore predicativo di un nome o nesso nominale. ${ }^{12}$ Tale participio è riconducibile a un atto di apposizione di un'etichetta denominativa: qualcuno firma, ossia mette un nome, a qualcosa.

Oltre a firmato e targato, i più ricorrenti, vi sono anche griffato ed etichettato:

(4) È la prima volta che succede dai tempi di Modugno: Eros Ramazzotti, già ruspante ragazzone di Cinecittà ora griffato Armani, è riuscito ad arrivare nelle classifiche di vendita di "Bill board", la bibbia della musica Usa. (CoLFIS, quotidiano)

(5) Sainsbury "si sta impegnando per la rimozione di GM dal cibo animale," la Co-op sta tentando di assicurare che "nessun prodotto etichettato Coop sia derivato da animali nutriti con mangimi contenenti colture GM" [...]. (CORIS MON2001_04)

Il participio passato si accorda per genere e numero al nome che modifica predicativamente; sia in maniera diretta, a mo' di attributo ( 1 e 2), sia in quanto parte nominale del predicato in un costrutto con copula (3). Il costrutto esprime una predicazione secondaria funzionalmente equivalente a una proposizione relativa (cf. Egerland 2011): "gli alberghi [che sono] targati Fiat".

In alcuni casi il nesso targato Nmc appare graduabile, mostrando con ciò la corrispondenza funzionale con un aggettivo qualificativo (cf. Vaxelaire 2005: 217):

(6) [...] il segretario generale della Cgil, Sergio Cofferati, ha invitato il governo a scoraggiare la cessione di Tim a Tecnost. C'è da dire però che quest'ultima, dopo l'operazione, si troverebbe ad avere un azionariato meno targato Olivetti, e forse un bilancio più leggero in fatto di debiti $[\ldots]$. (LaR)

12 Tale struttura ricorre pure con antroponimi, in particolare patronimici, come mostra 1'es. (2): “firmato Agnelli”. 
È però opinione comune che in realtà Baudo alla casa madre potrebbe tornare in occasione del prossimo Sanremo: un grande appuntamento che va in onda su RaiUno, ma non è così targato Rai come altre produzioni di varietà [...]. (LaR)

$\mathrm{Ci}$ sono esempi in cui il participio che precede il nome commerciale non presenta fenomeni di accordo rispetto a un nome:

(8) La Pepsi si è distinta tra tutte le altre compagnie e industrie che si sono scatenate nella pubblicità in onore del papa. [...] Più sotto ancora, l'esortazione: "Sappiamo interpretare il messaggio morale di questa visita di Sua Santità", firmato Pepsi. (LaR)

Quando ricorre in isolamento, come in questo brano (e si ricordi lo storico "firmato Diaz"), il costrutto non modifica un nome, bensì esplicita l'entità enunciatrice che, nel caso di (8), firma un messaggio, ingenerando quindi un'interpretazione passiva ('ciò è firmato da Pepsi'). ${ }^{13}$ Diversamente dagli esempi precedenti, questo tipo di struttura - che ricorre a basse frequenze con nomi commerciali - si colloca al livello dell'enunciazione (cf. Benveniste 1956). Qui non lo si approfondirà ulteriormente.

\subsubsection{Modificazione denominativa (con nomi riferiti a prodotti)}

Tipico è l'uso di firmato in combinazione con marchionimi che sono nomi (spesso patronimici) di stilisti e rappresentano, appunto, delle firme:

(1) Tra i selezionatissimi ospiti anche l'immancabile top model Naomi Campbell. Abito corto e trasparente color crema firmato Prada, è arrivata a palazzo Volpi insieme a Vittorio Cecchi Gori. (LaR)

(2) Tradotto in soldoni vuol dire più o meno questo: un tailleur firmato Armani costa, a New York circa mille dollari (due milioni). (LaR)

13 Questo caso non è dissimile da costrutti esplicitamente passivi: "si chiede il sequestro cautelativo delle azioni della Sme ritenendo valido ed efficace il contratto firmato dalla Buitoni stessa e dall'Iri" (LaR). Negli altri brani citati, in particolare laddove il nome modificato non si riferisce a un prodotto, non è possibile inserire la preposizione $d a$ prima del nome commerciale, rendendo il costrutto esplicitamente passivo. 
(3) Sordi e Tognazzi, Manfredi senza il noto pullover firmato Missoni (ma, curiosamente, in giacca e cravatta nocciola) e Villaggio-Verdone-Montesano a garantire allegria alla serata... (LaR)

(4) Monsignore yuppie: gioca a tennis, fa footing; [...] d'estate porta magliette firmate "Lacoste"; mastica chewing-gum [...]. (CoLFIS, quotidiano)

(5) Ancora tavole vestite a festa con i tessuti rosso fuoco firmati Bassetti su cui campeggiano foglie dorate [...]. (CoLFIS, quotidiano)

Nei brani citati il participio esplicita ciò che è incluso nel nome stesso, che è appunto una firma, e modifica insieme al marchionimo nessi nominali riferiti a prodotti, soprattutto capi d'abbigliamento.

Tuttavia l'uso di firmato Nmc si estende pure a nomi commerciali di settori merceologici diversi, in particolare quello alimentare:

(6) Ci sono cibi "firmati” Coop, GS, Fidel (un marchio di Esselunga). Come sono? (CORIS STAMPAPeriodici)

(7) Per arricchire una macedonia, inventare un irresistibile mangia e bevi, farcire delle crêpes dolci modello estivo, c'è ora il nuovo gusto di Carte d'Or - il gelato firmato Algida - Yogurt e Frutti di Bosco. (CoLFIS, periodico)

Le virgolette in (6) segnalano appunto che si tratta di un uso traslato. Targato ricorre in contesti comparabili, come modificatore di nomi riferiti a calzature, sigarette, veicoli:

(8) Il traguardo da raggiungere entro un paio d'anni è clamoroso per un'impresa nata appena cinque, sei anni fa: oltre 5 milioni di paia di scarpe targate Geox. $(\mathrm{LaR})$

(9) Quincy Watts non rifiutava una valchiria in cambio del suo paio di scarpette targate Nike? Quello era lo spot realizzato negli studi della Warner Bros [...]. (LaR)

(10) Rino Formica, ministro socialista delle finanze italiane, guida la crociata contro la multinazionale americana dai mille tentacoli. Il divieto temporaneo di importazione, distribuzione e vendita delle sigarette targate Philip Morris scatena un prevedibile putiferio. $(\mathrm{LaR})$

(11) Ma il problema non è affatto di natura tecnologica. È semmai legato ai costi. I computer targati Sony, pieni di soluzioni multimediali ideate proprio per 
distinguerli da quelli della concorrenza, si sono infatti rivelati un flop perché costavano troppo cari. (CORIS STAMPASupplementi)

(12) Ci sono stati momenti di grande tensione fra gli allevatori con le camicie a quadri sui loro colossi targati Fiat e John Deere e polizia e carabinieri, che non sono intervenuti. (LaR)

Con i nomi commerciali che ricorrono in (6-12), che non sono firme o targhe in senso stretto, l'uso dei participi targato e firmato mette in rilievo un processo attinente all'apposizione di un nome: un processo di denominazione. Il nome commerciale, al contempo nome d'impresa e di marca, soggetto e oggetto del processo denominativo, è l'entità che firma o targa il prodotto dandogli un nome, il proprio nome. In altre parole, la struttura qui delineata ha uno spiccato carattere di predicazione denominativa: essere "targato Fiat", "firmato Armani" e simili vuol dire recare scritto, in maniera più o meno metaforica, un nome proprio. Il nome commerciale è allora interpretabile come predicazione nominale mediata e introdotta dal participio.

Nei costrutti citati in questo paragrafo l'elemento modificato è un nome concreto, riferito a oggetti di consumo: gelato, magliette, cibi ecc. Sono contesti che abbiamo ritrovato nel $\$ 2.2 .1$ relativamente a usi predicativi del nome commerciale (il costrutto Det N[prodotto] Nmc): "scarpe Geox", “computer Sony" ecc.

(13) Ma proprio dalla Germania, dopo il recente lancio del 12 cilindri $B M W$, stanno per arrivare le novità più interessanti con un nuovo 12 cilindri targato Mercedes e un sofisticato tre litri sei cilindri dalla Opel. (LaR)

In questa proposizione ricorrono entrambe le strutture, funzionalmente equivalenti: nome commerciale $(B M W)$ in funzione predicativa e struttura targato Nmc (Mercedes) in funzione anch'essa predicativa rispetto al nesso nominale " 12 cilindri". In certi casi, dunque, il participio - sia firmato che targato - non pare necessario alla compiutezza del costrutto, in particolare quando segue immediatamente il nome comune modificato: nel brano da cui è tratto l'esempio (4), "porta magliette firmate «Lacoste»", si legge pure "indossa magliette «Lacoste»". Esigenze di variatio e altri aspetti contestuali possono influire sulla scelta dell'una o dell'altra struttura: 
(14) Quanto a Madonna, ha scelto ancora una volta il made in Italy (ma probabilmente senza saperlo) per i suoi primi occhiali da vista, per leggere a 41 anni: un modello semplicissimo e superminimalista dalla montatura in filiforme metallo firmato Ralph Lauren ma realizzato dall'italianissima Sàfilo. (LaR)

In questo caso si instaura una contrapposizione tra entità denominatrice ed entità produttrice, tra chi dà il nome di marca all'oggetto (in quanto designer: Ralph Lauren) e chi lo realizza materialmente senza che ciò si rifletta nella denominazione del prodotto (Sàfilo).

Ancora, il nesso "participio passato $+N m c$ ", eventualmente combinato ad altri modificatori, si presta particolarmente a fungere da predicato di forma nominale:

(15) Un altro Zero sta arrivando sul mercato delle gomme ed è targato naturalmente Pirelli. Accanto a uno pneumatico per supercar mitico come la P Zero, in questi giorni sta debuttando sul mercato la Scorpion Zero. ${ }^{14}$ (CORIS STAMPASupplementi)

(16) "Oggi ogni computer sembra targato Microsoft, anche se viene dalla Dell, dalla Compaq o dalla Gateway" ha detto Joe Klein [...]. (LaR)

\subsubsection{Usi metaforici}

Si è accennato (§4.5.2) al fatto che il processo di denominazione di cui è questione con i predicati targare e firmare va inteso, secondo i casi, come più o meno metaforico. Con nomi relativi a prodotti di una marca l'allusione a un marchionimo che funge da etichetta, da "targa", ha valore pressoché letterale; con altri nomi, non riferiti a prodotti, si avrà invece un'interpretazione metaforica (cf. anche gli ess. 6 e 7 nel §4.5.1).

(1) Solo nei prossimi giorni si capirà cosa sta succedendo a Radice Fossati, e s'inquadrerà meglio la storia delle bustarelle targate Fiat. (LaR)

(2) Stavolta nell'inchiesta entra il nome dell'Iveco, l'azienda Fiat del trasporto pesante, fornitrice per miliardi della Azienda trasporti municipali di Milano:

14 Si noti, en passant, il genere femminile dei nomi commerciali "P Zero" e "Scorpion Zero", di contro al nome comune generico "pneumatico" (v. §1.1.4). 
sarebbero appunto targati Iveco una buona parte dei miliardi finiti sul conto svizzero del Psi. (LaR) ${ }^{15}$

(3) Non molto meglio è andata con Patrick Kluivert, che in un altro poster targato Nike dice "Venables, dimettiti adesso". (LaR)

(4) Da febbraio nascerà il treno targato Alitalia. Si salirà in carrozza a Firenze e si finirà direttamente sul Jumbo per New York. (LaR)

La struttura è utilizzata qui non per designare un produttore in relazione al suo prodotto, bensì per dare un nome, un responsabile o un proprietario, a un oggetto: sono persone attive presso Fiat e Iveco ad aver versato milioni e bustarelle.

Il valore metaforico della modificazione si osserva ancora meglio nel caso di nessi nominali astratti che indicano processi o eventi:

(5) [...] in molti già vogliono eleggere l'esperimento dell'autore newyorkese come la panacea per fronteggiare lo tsunami digitale targato Kindle e iPad. Ma Safran Foer non ci sta a farsi incoronare controrivoluzionario [...]. (CORIS MON2008_10)

(6) Ma soprattutto c'è la potenza del brand: l'uscita di un cellulare marchiato Facebook susciterebbe un clamore paragonabile solo a quello delle iniziative firmate Apple e Google. (CORIS MON2008_10)

(7) Quello targato Ericsson non è il primo tentativo di dare un'estensione elettronica e senza fili ai cartelloni pubblicitari. (CORIS MON2001_04)

(8) Rialzo targato FIAT e GENERALI (CoLFIS, quotidiano [titolo])

(9) Tutto sembrava definito la scorsa settimana con la bocciatura del piano di privatizzazione targato Pirelli e con la denuncia del patto di sindacato. (LaR)

(10) Nello show rutilante della moda, vanno in scena venti minuti di autentico rigore, firmato Prada. Accompagnato da una musica lieve e ripetitiva, si fa strada un maschio cerebrale, tutto da scoprire [...]. (LaR)

Il nome commerciale che ricorre in questi esempi ha valore (pragmatico) di soggetto - di responsabile o iniziatore - rispetto al nesso nominale modificato. "Lo tsunami digitale targato Kindle e iPad" (5), per es.,

15 Il nesso "targati Iveco" funge qui da predicato nominale. 
equivale a "lo tsunami digitale provocato da Kindle e iPad", mentre il tentativo "targato Ericsson" (7) è un tentativo messo in opera da Ericsson. Anche nomi di entità umane possono essere modificati da targato

Nmc:

(11) Con un decreto che entrerà in vigore tra quindici giorni, il ministro socialdemocratico delle Poste Carlo Vizzini ha calato una rete di vincoli sulla pubblicità televisiva di vino, birra, superalcolici [...]. Il cowboy avventuroso o il velista spericolato, se "targati", presto dovrebbero sparire dai teleschermi. [...] Dice il ministro: "Vedrete, vedrete: il garante sarà rigido nell'applicare la legge. L'obiettivo politico è chiaro, non ci saranno cedimenti, nessun escamotage verrà tollerato. No, non credo proprio che quel cowboy targato Marlboro lo vedremo mai più...". (LaR) ${ }^{16}$

(12) Il giornalista targato Rai non è un giornalista dimezzato, è un giornalista con gli stessi diritti e gli stessi doveri dei colleghi della carta stampata. (LaR)

(13) "Questo accordo è stato l'ultimo colpo di coda di un ministro targato Fiat", ha detto Giacomo Garra (Forza Italia) riferendosi al fatto che il Trattato era stato siglato da Susanna Agnelli, ministro degli Esteri nel governo Dini. (LaR)

(14) Per quattro anni niente scritte (a meno di improbabili ripensamenti). Anche se renderebbe bene un Del Piero azzurro targato Fiat: secondo alcune stime dei pubblicitari la Figc incasserebbe oltre 20 miliardi all'anno [...]. (LaR)

(15) Lei [Pininfarina] è stato definito un presidente targato Fiat. (LaR)

(16) I dettagli dell'operazione Telettra-Telenorma Italia non sono ancora noti ma potrebbero anche sfociare nella creazione di una società ad hoc con Telettra che avrebbe un peso preponderante nel consiglio d'amministrazione mentre alla Telenorma andrebbe l'amministratore delegato. Il direttore commerciale sarebbe targato Fiat e quello del settore tecnologia Bosch. (LaR)

(17) Sogghignò Vittorio Ghidella (quand'era ancora targato Fiat) presentando la 164 al Palatrussardi: Fortuna che quelli del tout Milan non si sono accorti che i colori delle poltrone, blu e giallo, erano i colori di Torino. (LaR)

Anche in questo caso si ha una lettura metaforica del modificatore (predicato nominale in 16 e 17): targato Nmc indica una relazione tra il

16 Nel brano ricorre "targati" (tra virgolette) per alludere ai protagonisti di determinate pubblicità. Il participio passato presenta pertanto un significato specifico, lo stesso che si ritrova nel nesso targato Nmc. 
nome umano modificato e l'azienda o entità identificata dal marchionimo, relazione che si declina in vari tipi che qui non conta esplicitare perché immediatamente comprensibili nei vari contesti d'uso.

Una lettura metonimica si dà invece per il costrutto seguente:

(18) Ma per quanto "instant", il cinema è sempre più lento della tv e degli altri media e così il ritratto dei ragazzi firmati Timberland, Moncler, El Charro e Nai Oleari [sic] risulta già ampiamente usurato dagli sketch di "Drive In", dalla valanga di servizi usciti sui rotocalchi e persino dagli albi a fumetti come il volgarissimo "Il paninaro". (LaR)

A essere firmati $N m c$, infatti, sono i capi d'abbigliamento che i ragazzi indossano (e si veda inoltre "già ruspante ragazzone di Cinecittà ora griffato Armani” nell'es. 4, §4.5.1).

Parallelamente a quanto osservato nel $\S 4.5 .2$ per costrutti quali "scarpe (targate) Geox", anche nel caso di nomi [+ umani] a volte si riscontra coincidenza tra il costrutto $N$ targato $N m c$ e l'uso predicativo semplice $N$ Nmc. Si consideri l'esempio (19), da correlare ai casi di "uomini Fiat" riportati nel §2.2.3:

(19) È fondamentale sapere a chi sono intestati i conti, da quanto tempo sono aperti, quali sono stati i movimenti di denaro: insomma, è possibile che, per esempio, i cinque uomini targati Fiat - quattro manager più un concessionario Iveco decidessero di pagare le mazzette in autonomia, senza avvisare i vertici di corso Marconi? (LaR)

Il nesso targato Nmc si presta inoltre a esprimere un rapporto di sponsorizzazione da parte di una ditta o un marchio: ${ }^{17}$

(20) Per qualcuno, infatti, la mostra di Palazzo Grassi targata Fiat è un'apertura di stagione realmente storica. Una grande, raffinata rassegna dell'arte moderna. (LaR)

(21) I più fortunati d'estate andavano al Forte, spiaggia frequentata dagli Agnelli e dalla Milano industriale, d'inverno puntavano sulle nevi targate Fiat del Sestriere. (LaR)

17 Si veda anche "I polacchi una volta dovettero rimandare a casa Jaak Wszola, campione di salto, perché indossava scarpe giapponesi mentre l'équipe polacca era tutta sponsorizzata Adidas" (LaR). 
(22) Ci sarà anche un bosco targato Jaguar nella nuova foresta nazionale che sta sorgendo nel cuore dell'Inghilterra. La prestigiosa fabbrica di automobili di lusso contribuirà con 150 mila sterline $[\ldots]$. (LaR)

(23) Ma se accetta, Esquier oltre alle sue capacità potrebbe portare anche una bella base ad Auckland per il team italiano targato Prada. (LaR)

(24) In omaggio allo sponsor ufficiale, targato Perugina, verrebbe spontaneo dire, con Letta, che "tra le tv, dalle intese si è ormai passati ai Baci..." (LaR)

Oltre che come modificatore di nomi, il nesso targato Nmc compare pure con funzione di predicato nominale, come già visto in precedenza:

(25) La rivoluzione fu targata "Fiesta" (le "1.4" costavano come le "1.1") e firmata proprio da Massimo Ghenzer, che era divenuto presidente della Ford nel 1989 [...]. (CoLFIS, periodico)

(26) [...] l'anno scorso una grande campagna pubblicitaria (vincente) nel paese del Sol Levante si è conclusa con un grande Swatch di 150 metri "abbracciato" ad uno dei grattacieli di Tokyo. Ma non è solo targato Swatch il successo degli orologi svizzeri, è la riscossa di un intero sistema produttivo. (LaR)

(27) È molto probabilmente targata Fiat Mirafiori la nube maleodorante che da quasi un mese si aggira minacciosa su Torino. (LaR)

(28) [...] nasce il più grande gruppo mondiale nel campo dei sistemi di produzione per l'industria automobilistica. Nasce targato Fiat e tutto questo - fatto non trascurabile - avviene tra l'Italia e gli Stati Uniti. (LaR)

(29) Un'altra grande privatizzazione nei Paesi dell'Est dopo il passaggio della Skoda alla Volkswagen di un anno fa. Parte targata Fiat e ha come terreno di sperimentazione l'industria automobilistica polacca [...]. (LaR)

Il nesso funge da predicato nominale in (25-27) e da complemento predicativo del soggetto in (28-29).

Rispetto agli esempi in cui il costrutto targato/firmato $\mathrm{Nmc}$ modifica un nome relativo a un prodotto ( $\$ 4.5 .2)$, nei contesti dove esso ricorre con valore metaforico il participio passato ha un minor grado di ridondanza e, soprattutto nei costrutti di tipo copulare o con complemento predicativo del soggetto appena visti, non è pensabile tralasciarlo. 


\subsubsection{Non solo targhe, non solo firme. L'esempio di calzato}

Infine emergono dai dati alcuni casi particolari, indici del fatto che il modulo "participio passato $+N m c$ " si può estendere a verbi diversi da quelli visti sinora, che rientrano tra i "verbi supporto" delineati da Grass (2002). Ecco due esempi:

(1) Se non fosse che l'anno scorso, 1994, Lisbona è stata la capitale europea della cultura, diremmo che il calendario si è fermato ai Settanta. [...] Con i lustrascarpe che imprecano perché i turisti, ahimè, son tutti calzati Adidas, Reebok, Haed [sic] o, al massimo, in corda. (LaR)

(2) [...] la domenica ogni gioco era rigorosamente vietato da Dio e dagli uomini. [...] Ma fu soltanto il lunedì, sotto un cielo meno brillante, che misi finalmente il mio piede calzato Superga sul campo numero 16 del Club. (LaR)

Dei turisti (o dei piedi) calzati Nmc si può dire che "calzano Nmc", con un uso avverbiale del nome commerciale. In entrambi i costrutti esso ha funzione di modificatore; non più di tipo denominativo, come per "computer targati Sony", e neanche metaforico, come nei casi di "rialzo targato Fiat" o "cowboy targato Marlboro". Un costrutto comparabile sarebbe per es. "i turisti indossano delle Adidas", in cui il marchionimo ricorre come nome, in funzione argomentale.

Resta da approfondire se questi usi ricorrano anche con altri verbi e nomi commerciali. In ogni caso si tratta di ricorrenze meno frequenti rispetto agli esempi di targato e firmato qui riportati, i quali sono utilizzati anche con valore metaforico, che per calzato sembrerebbe meno probabile.

\subsection{Alla Renault volevano Prost. Nessi preposizionali e soggetto non specificato}

In una struttura relativamente frequente, eppure - a conoscenza di chi scrive - mai menzionata negli studi linguistici sui nomi commerciali, il nesso preposizionale alla $\mathrm{Nmc}$ si combina a un predicato verbale di terza persona plurale con soggetto non manifesto: 
(1) Nonostante le furbate, alla Pirelli storcono il naso. (LaR)

(2) La nuova Williams non sarà in pista subito. Il debutto è previsto a luglio (Magny Cours), ma non è detto che non si possa anticipare. Per questa vettura alla Renault volevano Prost. (CoLFIS, quotidiano)

(3) il Torino rischia di sparire, bisogna trovare un nuovo padrone. [...] I grandi nomi si negano, niente Ferrero o Cinzano, mentre alla Lavazza lo dicono chiaro: "Il Toro non ci serve, non è un veicolo pubblicitario utile". (LaR)

\subsubsection{Con verba dicendi}

I verbi che compaiono in questa struttura sono vari; $\mathrm{i}$ più frequenti, peraltro, sono verba dicendi:

(1) Il rodaggio è andato bene, dicono alla Fiat, anche se le piccole variazioni tese a perfezionare il nuovo documento erano indispensabili. (LaR)

(2) "Secondo i nostri calcoli nei prossimi anni la domanda di pneumatici super-ribassati crescerà del 50 per cento e in questo senso non temiamo confronti dicono alla Pirelli-. [...]”. (LaR)

(3) Ma, per finire, la Punto ucciderà la Uno? "Neanche per idea", giurano alla Fiat, "la Uno vivrà ancora due anni almeno....". (LaR)

(4) Nei quartieri generali delle due case europee si fa di tutto per minimizzare la portata dell'intesa. È solo un accordo per la cessione alla Michelin di cespiti e attività che non ci interessano, affermano alla Pirelli. Non ci sono altre intese, accordi di altro tipo, confermano anche alla Michelin. (LaR)

(5) Buona parte dei benefici ottenuti tramite il piano di ristrutturazione, sottolineano alla Pirelli, saranno vanificati dal maggior costo del denaro. (LaR)

(6) "In realtà", spiegano alla Lavazza, "in questi ultimi anni il concetto di "normalità' all'interno della famiglia si è molto allargato. E la pubblicità si limita a prenderne atto". (LaR)

(7) "Ristrutturare l'indebitamento come reazione a interventi d'emergenza sarebbe sbagliato", commentano alla Barilla, "perché in momenti simili le indicazioni sono spesso contraddittorie". (LaR) 
(8) I concorrenti della Buitoni nell'acquisto della Sme stanno a guardare. Avete ricevuto richieste di tangenti? "Per carità, assolutamente no, né oggi né mai", rispondono alla Barilla. (LaR)

Non si tratta di casi eccezionali: nel corpus LaR il solo "dicono alla Fiat" ricorre 34 volte. La struttura Nmc dice, al confronto, è più rara: nel corpus LaR i casi pertinenti con Fiat sono meno di dieci.

La struttura analizzata ricorre come un inciso in cui il predicato solitamente precede il nesso introdotto dalla preposizione $a$, il quale non ha funzione di oggetto indiretto legittimato dal predicato. Lo si può osservare nell'incipit del brano seguente, in cui ricorre un oggetto indiretto $(m i)$ :

(9) "Lo sa cosa mi hanno detto alla Lavazza? Mi hanno detto 'Nino, noi ti vogliamo bene e ti rispettiamo. Fai quello che ti senti'. E se mi candido con Pannella che succede?, gli ho fatto io. Mi sostituite? Sa cosa mi hanno risposto? 'Piuttosto chiudiamo la Lavazza'. Pensi che carini”. Carinissimi alla Lavazza. Nemmeno l'ombra della censura, nessun cenno alle battute che già circolano sul 'caffè radicale'. (LaR)

Caratteristica del costrutto è l'assenza di un soggetto manifesto per il predicato verbale di forma finita, soggetto che non è possibile recuperare dal contesto (il brano 9, per es., apre un articolo di giornale). ${ }^{18}$ Vi sono alcune ricorrenze del costrutto con preposizione in:

(10) È stato annunciato in settimana un incontro tra lo stesso Biaggi e i dirigenti dell'Aprilia per il rinnovo del contratto. "La firma è in cima ai nostri pensieri" dicono in Aprilia. (LaR)

(11) [Il Marengo] È un veicolo - giurano in Fiat - che si colloca ai vertici della sua categoria per quanto riguarda la portata utile, che è di 570 chili, conducente compreso [...]. (CORIS STAMPAQuotidiani)

(12) L'assessore alla cultura Carrubba li sta corteggiando da un paio d'anni: "Ho trovato la sede, la Fiera. E i partner: Regione e Fondazione Cariplo, cui ora si aggiunge Mediaset. Ma dagli editori non ho ricevuto risposte precise". Replicano in Mondadori: "Se n'è parlato molto, ma non abbiamo mai visto un progetto. Quando ci sarà, Mondadori sarà interessata". (LaR)

18 Una variante con soggetto manifesto (benché non meglio specificato): "Questi operai, dicono quelli della Honda, sono il nostro fiore all'occhiello” (LaR). 
(13) È una società messa all'asta, non potete farlo, sottolineano i legali della cordata Iar. E in Barilla aggiungono: il nostro intento comunque non è quello di aprire un nuovo contenzioso. (LaR)

È la versione avanzata, dell'italiano tendenziale, di dicono alla $\mathrm{Nmc}$, in cui il nome commerciale non è determinato e, correlativamente, entra in uso la preposizione in (v. §3.4.1). ${ }^{19}$

\subsubsection{Con altri predicati verbali}

(1) Dieci, cento, mille spaghetterie. Il progetto è ancora top-secret ma alla Barilla ci stanno lavorando alacremente. (LaR)

(2) Alla Apple chiamano periodi come questi «fast forward», avanti tutta, come quando tieni premuto il pulsante dell'iPod. (CORIS MON2005_07)

(3) [...] a Monza tre Guzzi arrivarono ai primi tre posti e la stampa specializzata gridava allo scandalo per l'eccessiva supremazia tecnica delle moto italiane che vincevano tutto quello che c'era da vincere. Ma non contenti alla Guzzi andarono avanti e polverizzarono letteralmente la concorrenza [...]. (LaR)

(4) Gli specchietti esterni sono "scomparsi": non solo perché peggiorano l'aerodinamica, ma anche perché alla Renault sono convinti che, in determinate condizioni di maltempo e per disporre di un angolo di visuale più ampio, sia meglio ricorrere alla tecnica delle telecamere. (CoLFIS, periodico)

(5) Negli stessi giorni e momenti, in tutta Italia, un gran fiume cola via incessantemente dalle condutture invecchiate e malconce di tante reti idriche. Milioni di metri cubi d'acqua si vanno continuamente disperdendo ogni anno, unicamente per insufficiente o inesistente manutenzione, largamente propizia anche al furto. È come se alla Fiat buttassero via un'auto nuova ogni tre; o se la lasciassero rubare. (CORIS EPHEMLettere)

19 In Spendere Meglio ricorrono inoltre casi di presso Nmc (cf. §3.4.1): "Presso Schwarzkopf si afferma che, se utilizzate correttamente, le tinture per capelli sono sicure per chi le utilizza" (SM 6/07); "Anche presso Sony Ericsson ci si dice lieti delle configurazioni dei provider" (SM 6/07). I predicati di forma impersonale sono dovuti probabilmente all'influsso del tedesco, dato che gli articoli della rivista sono spesso traduzioni da questa lingua. 
Negli esempi ricorre, con predicati verbali diversi, la struttura vista nel paragrafo precedente (“dicono alla Pirelli”). Con una differenza: la maggioranza dei casi riportati nel §4.6.1 fungeva da inciso, menzionando l'enunciatore di un messaggio riportato nel testo in forma di discorso diretto; nei costrutti appena citati, invece, il nesso alla Nmc è seguito da un nesso verbale complesso (con oggetti diretti, subordinate relative e così via).

Il nesso preposizionale alla $\mathrm{Nmc}$, con nome commerciale al femminile, svolge in apparenza funzione locativa (v. §3.4.1). Funzionalmente, però, esso equivale a un soggetto:

(6) Sorride Nanni Tua, presidente di una piccola fabbrica di sci (110 mila paia all'anno) ad Occhieppo Superiore vicino Vercelli. Ma sorridono anche alla Diadora, 190 miliardi di fatturato 30 dei quali nell'abbigliamento invernale. (LaR)

L'esempio (6) illustra tale equivalenza: da una parte "Sorride Nanni Tua", dall'altra "sorridono anche alla Diadora". In quanto fabbrica o azienda, Diadora non può sorridere, se non per metonimia; a sorridere è allora un soggetto alla terza persona plurale, un "umano non specificato", riferibile a persone che lavorano per l'azienda definita attraverso il nome commerciale. Lo stesso vale per i costrutti citati in precedenza.

\begin{abstract}
Pur non felici per quel che accade a Wall Street, alla Fiat tendono a sdrammatizzare. La nostra - dicono - era concepita come una iniziativa di medio-lungo termine: il successo non può essere misurato in poche settimane. E aggiungono che il suo scopo principale era quello di attrarre quei grandi fondi pensione degli Stati Uniti (1900 su 2000) che hanno restrizioni statutarie nell'operare all'estero. (LaR)
\end{abstract}

Nel brano (7) il soggetto non specificato di terza persona plurale ricorre più volte, in relazione a uno stesso costrutto alla $\mathrm{Nmc}$; tanto con verba dicendi quanto con altri predicati verbali.

\title{
4.6.3 Osservazioni sulla struttura
}

Nel $§ 4.6 .2$ si è affermato che il nome commerciale preceduto da alla svolge in questi costrutti una funzione locativa. Più che identificare l'esatto significato del nesso preposizionale, però, conta osservare eventuali rapporti di equivalenza con altre strutture. 
Al proposito si ritorni ai casi, illustrati nel $\S 4.6 .1$, in cui un nome commerciale è legittimato come soggetto da un verbum dicendi: "Cablecom dichiara...", "la Nissan ci suggeriva...". Si tratta, come si è osservato, di una metonimia: il nome commerciale, considerabile in questi contesti come nome d'impresa (in particolare per il valore combinatorio assunto dall'articolo la; cf. §1.1.2), vale per le persone che vi lavorano. Di qui il tratto [+ umano] riconoscibile nel soggetto. Parimenti, anche con le strutture "dicono alla $N m c$ " e "alla $N m c$ + verbo" il soggetto - non manifesto, ma spesso [+ umano] - è in relazione al nome dell'impresa, la quale presenta un'agentività non attribuibile a un referente umano particolare. Entrambi i costrutti permettono di non specificare ulteriormente il soggetto, in quanto non rilevante; a essere ritenuto pertinente, per contro, è il nome di marca, dell'azienda di cui è questione. Per questo si ritiene che il nesso alla $\mathrm{Nmc}+$ predicato verbale sia da analizzare come struttura peculiare dei nomi commerciali.

Anche se dal punto di vista del valore complessivo i costrutti (la) $\mathrm{Nmc}$ dice e alla Nmc dicono si equivalgono, nondimeno essi mettono in risalto due aspetti diversi correlati al nome commerciale. Riprendendo Grass (2002: 228), si osserva che in (la) Nmc dice emerge il valore del nome di marca, riferito all'impresa, come entità umana (per metonimia), come "umano collettivo", in quanto può fungere da soggetto di predicati che legittimano soggetti umani, mentre con alla Nmc dicono viene in rilievo piuttosto il valore di toponimo, di luogo (fisico o astratto) in cui si prendono decisioni e si fanno affermazioni.

Chiaramente la struttura alla $\mathrm{Nmc}+$ predicato verbale di terza persona plurale va distinta da quella in cui il nome commerciale introdotto da alla ha valore locativo, senza peraltro svolgere una funzione che si potrebbe definire - ci si passi la boutade - di "soggetto indiretto": un soggetto che non è tale grammaticamente e nella forma (non, ovviamente, nella funzione) ricalca le parvenze dell'oggetto indiretto.

perché ho un tecnico che mi sta mangiando sotto gli occhi e ora se questo è il sistema di fare radio alla $R A I$ non lo farebbero io mi chiedo ma alla RAI mangiano $*[\ldots]$ no mangiano da altre parti alla RAI (LIP FB 35)

In questo esempio, alla Rai mangiano non trova un equivalente in la Rai mangia, come avviene invece per i casi di "alla Fiat dicono"/“la 
Fiat dice". In questa specifica combinazione di nome commerciale e predicato il marchionimo ha valore esclusivamente locativo; non gli si riconosce l'agentività che caratterizza i casi visti sinora.

Similmente, nei casi in cui il predicato ha un soggetto manifesto (negli esempi 2 e 3, "i rimborsi spese" e "gli assaggiatori") non si ritrova la struttura di cui è questione:

(2) D'altronde era indispensabile, visto che alla Bayer i rimborsi spese rappresentano una fetta notevole del fatturato [...]. (CoLFIS, periodico)

(3) eccoli qua gli assaggiatori vedete * eccoli qua vedete * \#* giorno dopo giorno alla Yomo verificano la qualità della produzione (LIP MD 18)

Nel primo caso non vi è un costrutto equivalente con soggetto "la Bayer"; per il secondo, invece, è pensabile un parallelo con il costrutto "la Yomo (= i suoi assaggiatori) verifica la qualità della produzione", in cui il nome commerciale abbia valore metonimico.

\section{Conclusioni}

L'analisi svolta in queste pagine mette in evidenza il ruolo delle correlazioni all'interno della proposizione e la loro importanza nel determinare le proprietà dei nomi commerciali.

Innanzitutto si è osservato che, per i nomi commerciali con funzione grammaticale di soggetto ( $\$ 4.1)$, è la correlazione con un predicato verbale che legittima un soggetto umano a far scaturire l'interpretazione [+ umano]. Nomi come Opel o Barilla non sono umani a priori; sono riconoscibili come tali solo relativamente a un contesto frasale.

La determinazione permette di differenziare le strutture in cui ricorrono nomi commerciali, come già visto per i nessi nominali (cap. 1). Se con la funzione argomentale di soggetto (\$4.1) si riscontra in genere variazione libera tra nome determinato e non-determinato ("La Migros dice" = "Migros dice"), per l'oggetto diretto, o meglio in posizione postverbale ( $\S 4.2$ e 4.4), non vale la medesima cosa: tra fumare Marlboro e fumare una Marlboro non vi è perfetta equivalenza. L'uso 
non-determinato si correla a una funzione avverbiale del marchionimo; si tratta quindi di un uso predicativo, non argomentale (tant'è vero che vi ricorrono pure predicati verbali che solitamente non reggono oggetti diretti). Per questo motivo, sviluppando uno spunto di Folena (1964: 119), si può sostenere che il passo da sorriso Durban's a sorridete Durban's è breve perché si tratta, in entrambi i casi, di usi predicativi del nome commerciale. È peraltro necessario considerare anche il soggetto della proposizione: se è un altro nome commerciale, l'alternanza tra forme con e senza articolo è libera ("Eridania ha acquistato Carapelli" non è come "mia zia compera solo Barilla").

I nomi commerciali hanno funzione predicativa quando ricorrono in costrutti copulari ( $\$ 4.3)$; in particolare si avvicinano funzionalmente agli aggettivi quando non sono determinati (§4.3.2: "Il fatto che [il cacao] sia Ferrero").

Quanto alle strutture "volare Alitalia" (§4.4), "firmato Armani" (§4.5), "alla Fiat dicono" (§4.6), si è notato che il primo tipo è stato studiato da vari linguisti, mentre per gli altri due non pare vi siano menzioni nella letteratura. Benché tali strutture non si configurino come particolarmente innovative o controtendenza, come avviene per la prima in quanto modulo largamente sfruttato dalla pubblicità, nondimeno la frequenza relativamente elevata con cui si presentano nei dati invita a considerarli in maniera approfondita.

Le strutture con participio passato ("targato Fiat") sono funzionalmente equivalenti a un aggettivo qualificativo. Esse fungono tanto da modificazione predicativa di un nesso nominale, quanto da parte nominale del predicato nei costrutti con copula. A livello proposizionale si tratta di una struttura morfologicamente più ricca rispetto al solo nome commerciale usato come modificatore: si veda "Il direttore commerciale sarebbe targato Fiat", con indicazioni di modalità sul verbo che si combina al marchionimo. L'uso in correlazione con nomi relativi a prodotti, d'altro canto, coincide funzionalmente con il semplice uso predicativo del marchionimo ("scarpe targate Geox" come "scarpe Geox").

Come per i modificatori di marca Nmc (§2.2.3), anche nel caso di "participio passato $+N m c$ " può avere luogo un'estensione dal senso letterale (oggetto che reca il marchio X) a quello traslato: "Y targato X" 
indica una relazione di $Y$ con la marca o l'impresa $X$, con il valore di "qui si riconosce l'azione o l'influsso di $\mathrm{Nmc}$ ".

Nella struttura "alla Fiat dicono" il nome commerciale equivale pragmaticamente al soggetto del costrutto "la Fiat dice", con valore metonimico. Viene in risalto non tanto la caratterizzazione [+ umana] del marchionimo quanto il suo essere un toponimo, sia pure in riferimento a un luogo astratto. Così una costruzione a soggetto implicito e con un nome commerciale che non è manifestamente [+ umano] può fare a meno del soggetto senza che ciò vada a discapito della comprensione.

L'analisi della proposizione apre il campo a studi che riguardano porzioni testuali ancora più ampie e applicano altre metodologie d'indagine. La comprensione dei nomi commerciali nell'uso linguistico non si esaurisce infatti con l'analisi sintattica ma dovrebbe includere una più globale analisi testuale. In questa sede ci si limita a indirizzare il lettore verso questo tipo di ricerca; più avanti, nel cap. 7 , si forniranno alcuni spunti di analisi riguardanti opere di narrativa in cui ricorrono nomi commerciali, con osservazioni che trascendono il puro dato morfosintattico a favore di una visione sistemica che tenga conto di molteplici elementi. 


\section{Barilliani o DeCecchisti? Derivazione e composizione}

\section{Introduzione}

La descrizione delle ricorrenze di nomi commerciali nei testi non sarebbe completa senza un accenno alle alterazioni morfologiche su base marchionimica. Si tratta, di necessità, di una trattazione breve e a titolo puramente esemplificativo, dato che il numero di types - da alfista a Deltona - è relativamente ridotto; d'altro canto, il tipo di ricerca condotto non si presta a un'analisi a tappeto di tali derivati, che peraltro non rientrerebbe negli obiettivi del lavoro. ${ }^{1}$ Esempi di derivati demarchionimici forniscono Schweickard (1992: 116-117) e Platen (1997: 129-138). ${ }^{2}$

Ferma restando la debole produttività derivazionale, che i marchionimi condividono con i nomi propri (cf. Jonasson 1994: 32), è soprattutto la pubblicità a servirsi di nomi derivati e alterati (v. gli esempi recati da Platen 1997: 131-132). Nelle tipologie testuali delle quali ci si occupa in questa sede vige perlopiù - come si è notato nei capitoli precedenti - la capacità dei nomi commerciali di manifestare nella proposizione funzioni diverse, in particolare aggettivali, senza che alla variazione funzionale corrisponda una variazione formale. Le eccezioni che forniscono materia al presente capitolo sono considerate anch'esse alla luce della prospettiva sintattica e combinatoria specifica di questa ricerca.

1 Si rammenta che la raccolta di dati è stata eseguita ricercando, in corpora preesistenti, una serie di nomi commerciali. I casi di derivazioni presentati in queste pagine sono emersi tangenzialmente oppure attraverso ricerche mirate di derivati precedentemente noti, ciò che rende l'analisi episodica e non esaustiva.

2 Platen (1997: 129n) distingue tra "internen Ableitungen" (come Calgonit da Calgon: da un marchionimo a un altro), delle quali non si occupa, e "allgemeinen deonomastischen Formen" (per es. scotcher da Scotch), che si caratterizzano in quanto mirano a integrarsi nel vocabolario. 


\subsection{Formazioni per affissazione}

Suffissi particolarmente produttivi, come del resto per i derivati deantroponimici, sono -iano e -ista (cf. Seidl 2004: 411). Con marche di automobili prevalgono le formazioni in -ista (cf. Platen 1997: 130):

(1) Una 145 amichevole, che non provoca rifiuti in tutti quelli che considerano le Alfa rigorosamente sportive. Per questo siamo sicuri che favorirà l'ingresso nella famiglia degli alfisti di altri e nuovi clienti. (CoLFIS, periodico)

(2) Interesse di squadra avrebbe voluto che Aghini cedesse la vittoria a Juha Kankkunen, l'altro lancista in lizza per il titolo iridato [...]. (CoLFIS, quotidiano)

Platen (1997: 130) osserva che in genere sono marchi nazionali di autovetture a presentare questa derivazione, dal momento che i singoli modelli mancano della comune Weltanschauung automobilistica che pare requisito necessario per neoformazioni come quella in (1).

Con i nomi di modelli specifici di veicoli a motore ricorrono invece spesso suffissi a valore alterativo:

(3) La cosiddetta "Deltona" è stata superiore per motore, tenuta di strada, freni e affidabilità. (CoLFIS, quotidiano)

(4) C'era "Lascia o raddoppia", il vespone e la mitica 600. (CoLFIS, quotidiano)

(5) Nel 1937, presso la Scuderia Ferrari, venne progettata e costruita una eccezionale monoposto, la famosa Alfa Romeo “Alfetta 158” [...]. (CORIS MISCVolumi)

Negli esempi (3-5) i marchionimi suffissati (sulla base rispettivamente di un nome di modello, nome di prodotto e parte di nome d'azienda) sono nomi non ufficiali - ma molto diffusi - riferiti a modelli: Lancia Delta HF Integrale, Vespa PX e Alfa Romeo 158.

Un altro suffisso ad alta produttività con i nomi di marca è -iano:

(6) [Il secondogenito di Luciano Benetton] è entrato (primo esponente della nuova generazione) nella stanza dei bottoni dell'impero benettoniano. (CoLFIS, periodico) 
Utilizzato come attributo modificatore del nome comune impero, l'aggettivo derivato da marchionimo corrisponde al marchionimo non modificato in funzione predicativa: la struttura $N(d i) ~ N m c$ identificata in precedenza. "Impero benettoniano" vale dunque "impero (di) Benetton".

Mentre in (6) l'aggettivo demarchionimico si presenta con valore neutro, come puro aggettivo relazionale, nell'estratto che segue i derivati con suffissi -iano e -ista indicano adesione partigiana, come già nel brano (1):

(7) È aperto il sondaggio. Siete più Barillisti o DeCecchisti? [...] Nessuna delle due, sono garofalista [...] Appartengo a famiglia di Barilliani da 3 generazioni $\left(\log ^{3}\right)$

L'invito a prendere posizione segue l'idea diffusa secondo la quale il comportamento nell'ambito dei consumi è un modo per esprimere attitudini profonde. La formazione di aggettivi (sostantivati) specifici dà luogo a creazioni in genere estemporanee e di poco momento, alla radice delle quali, però, si riconosce un modulo produttivo e duraturo.

Ricorrono di frequente pure i suffissi izzare, -izzato: ${ }^{4}$

(8) La nostra letteratura, e anche il nostro cinema (rispetto a quello Usa) si occupa poco o nulla di sport perché lo sport è sempre più macdonaldizzato. Intendo dire che lo sport impone ormai ritmi da fast food, è una religione del nostro tempo che si celebra e s'impone come spettacolo, come serie di eventi ravvicinati. (LaR)

Qui il valore metaforico della forma (cf. infra, cap. 6) è parafrasato nel brano stesso.

Infine, un caso di formazione verbale parasintetica (di uso comune) si legge in Moccia (2004: 200): "incellofanate".

$3<$ forum.alfemminile.com> (8.5.2012).

4 Il modello ricorre da lunga data in ambito commerciale: Odolisez-vous, con l'equivalente italiano, risale al 1912. Per Platen (1997: 131) si tratta di "fast schon stereotyp zu nennenden Bildungen". 


\subsection{Formazioni per composizione}

I nomi commerciali sono modificati, oltre che tramite affissazione, anche per giustapposizione di altri elementi (nomi, aggettivi ecc.):

(1) Sarà pur vero che il bello delle cabriolet è andare a spasso, ma non si spendono 213.250 euro soltanto per avere il cielo sulla testa e il vento nei capelli, anche se sull'Alfa $8 \mathrm{C}$ di cielo ce n'è di più e il vento della velocità è molto più forte. Questa super-Alfa è nata per essere un mito prima che un'automobile [...]. (CORIS MON2008_10)

(2) «Di certo sfrutteremo l'alleanza nei confronti della grande industria per fare contratti d'acquisto a livello europeo, come già avviene per le grandi catene internazionali» spiega Francesco Pugliese, il manager ex Barilla e Yomo che dal 2004 è direttore generale del Conad [...]. (CORIS MON2005_07)

Tanto in super-Alfa quanto in ex Barilla e Yomo gli elementi aggiunti al marchionimo funzionano come una sorta di prefissoide. In particolare si osserva che il nesso nominale "il manager ex Barilla e Yomo" (2) è parallelo al tipo $N[+h u m] N m c$ (v. \$2.2.3), con la differenza che la particella ex proietta un valore temporale di passato sulla relazione (di lavoro) che lega nome comune e marchionimo: Francesco Pugliese, in altri termini, è stato manager presso Barilla e Yomo.

Nei brani che seguono la giustapposizione riguarda un nome:

(3) Alla guida della Citroen-pirata, due Marocchini [...]. (CoLFIS, quotidiano)

(4) A quell'epoca ero gelataio ambulante a Torino: partii alle dieci della sera precedente con la mia Lambretta-furgoncino carica di gelato. (CORIS STAMPA Quotidiani)

In Citroen-pirata il nome comune è usato in funzione appositiva, con valore figurato. ${ }^{5} \mathrm{Nel}$ caso di Lambretta-furgoncino, altro composto endocentrico, il nome comune specifica il marchionimo e fa riferimento a una particolare linea di veicoli.

Non mancano composti univerbati: 
(5) Ma davvero l'America si è disintossicata dalla swatchmania? (LaR) ${ }^{6}$

(6) La possibilità che la Swatchcar possa essere costruita in Francia non piace naturalmente in Germania [...]. (LaR)

Il primo è considerabile un equivalente interpretativo di febbre da Swatch (§3.3), mentre il secondo è il corrispondente, in inglese, di un ipotetico "auto (della) Swatch", con nome commerciale in funzione predicativa.

\section{Conclusioni}

Negli esempi di queste pagine i derivati da marchionimi, che illustrano la forza innovativa di alcuni meccanismi di formazione delle parole, presentano in genere un significato determinabile, diversamente dalle derivazioni frequenti in ambito pubblicitario, che sono aperte a interpretazioni molteplici e creative (Platen 1997: 133 porta gli esempi "I swatch very much!" e "Je me rowente la vie").

In generale si osserva che le derivazioni mantengono perlopiù un'interpretazione referenziale, ovvero non si fondano su un eventuale significato metaforico del nome alla base, come avviene - a detta di Jonasson (1994: 34) - per i derivati di nomi propri (di persona). ${ }^{7}$ Un'eccezione è costituita da macdonaldizzato, un conio utilizzato, come si evince dalla spiegazione che segue ("lo sport impone ormai ritmi da fast food"), in riferimento a un valore predicativo-antonomastico di McDonald's. Di questi usi, più estesamente, sarà questione nel capitolo che segue.

6 Ricorre Swatchomania in un es. tedesco citato da Platen (1997: 130n).

7 "Si on trouve des dérivations comme donjuanesque, donjuaniser [...], les Npr qui leur fournissent une base morphologique sont tous lexicalisés avec un sens descriptif métaphorique." D'altronde esistono pure derivati deonimici come giovanneo o paolino, che non sono utilizzati con valore metaforico. 



\title{
6. Usi metaforici
}

\author{
Introduzione
}

(1) Durante il soggiorno obbligato nel villaggio di Superior il turista per caso, che non è certo un tipo regolare, incontra una quantità di gente molto più sballata di lui: un meccanico trucido e truffatore (Billy Bob Thornton), un vecchio indiano sentenzioso (Jon Voight), un giovanotto sbruffone con la sua Barbie scema (Joaquin Phoenix e Claire Danes), una coppia di rapinatori e una vecchietta messicana armata di fucile. (LaR)

Nel film di cui è questione in questo brano, al personaggio interpretato da Joaquin Phoenix non si accompagna, è ovvio, una bambola prodotta da Mattel. Il nome commerciale Barbie, seguíto dall'attributo scema, vale come designazione antonomastica, le cui implicazioni predicative si possono descrivere in questi termini: giovane donna, generalmente bionda, di una bellezza stereotipa e artificiale.

Questo capitolo è dedicato agli usi traslati o metaforici ${ }^{1}$ dei nomi commerciali, che, facendo leva su una o più caratteristiche salienti del referente originario, manifestano funzione descrittiva, non identificativa (cf. Jonasson 1991: 70-71). Il marchionimo, originariamente una predicazione metalinguistica (cf. La Fauci 2011: 213-214), acquista funzione di predicazione non-metalinguistica: nell'esempio (1) Barbie non è, e comunque non è necessario che sia, il nome della ragazza in questione. Si può parlare di usi antonomastici nella misura in cui tali nomi assurgono a esemplari prototipici di una categoria i cui membri si somigliano in quanto presentano una particolare caratteristica (cf. Jonasson 1991: 64).

Usi metaforici sono comuni con i nomi propri (cf. Wee 2006; Jonasson 1991): si pensi a una struttura predicativa come "Giovanni Allevi è il Federico Moccia della musica classica" o al significato che

1 Con "metafora" si intende un procedimento linguistico basato su una relazione di similarità (cf. Jakobson 1956). 
hanno acquisito toponimi come Vietnam o Auschwitz (cf. Jonasson 1991: 63; La Fauci 2011: 213).

Barbie è un esempio paradigmatico di nome commerciale cui è accaduto di "rientrare anche in modo estemporaneo con funzione di «appellativo» nel circuito dell'espressione linguistica, di perdere cioè la sua marcatezza metalinguistica" (La Fauci 2011: 214). Altri casi frequenti e noti ai parlanti sono McDonald's per "[p]roduttore e distributore a basso costo di beni di largo consumo", che ha dato origine a tutta una serie di derivati con prefissoide $m c$ - (Zardo 2007: 267n; cf. inoltre Vaxelaire 2005: 224-225). Ogni comunità linguistica o culturale ha i suoi esempi di nomi commerciali elevati al rango di antonomasie, utilizzati con valore predicativo in virtù del loro carattere evocativo e allusivo (sul quale cf. Zardo 1997: 33 e 2007: 266).

Dal punto di vista sintattico, Jonasson (1991: 64) osserva che i nomi propri usati metaforicamente, pur mantenendo in genere la maiuscola come marca grafica di proprialità, si contraddistinguono rispetto agli usi referenziali in quanto sono generalmente preceduti da un determinatore e spesso accompagnati da complementi. Dal profilo semantico, gli usi metaforici ripropongono l'annosa questione del significato dei nomi propri (cf. La Fauci 2011: 213), del loro "contenuto descrittivo", dal quale non si può prescindere nel caso di usi figurati quali "la sua Barbie scema" o "una nuova Auschwitz".

L'analisi sintattica proposta in questo capitolo si articola secondo il tipo di struttura in cui sono contenuti i marchionimi a valore metaforico. Nel fare ciò ci si ispira in parte alla classificazione di Jonasson (1991: 67), che elenca tre proposizioni caratteristiche degli usi metaforici con nomi propri: 1) "SN-être-SN" ("Il est le nouveau Don Juan"); 2) " $S N$, $S N$ " ("Mel Brooks, Don Quichotte du rire, ..."); 3) " $S N$ de $S N$ " ("le Molière de notre époque" ma anche "un Hamlet yiddish").

In questa sede si privilegia una prospettiva funzionale: il marchionimo è il nucleo del nesso nominale (uso argomentale) oppure funge da modificatore? Per il primo tipo si hanno a) gli usi come predicato nominale (§6.1.1): "il corpo umano è una Ferrari”; b) gli usi nominali modificati (§6.1.2): "una Barbie mediterranea". Per il secondo tipo si hanno invece: c) i costrutti $N$ Nmc ("lavoratori kleenex") e i costrutti $N N c N m c$ in cui il nome comune ha valore di classificatore: "reginetta 
modello Barbie" (§6.2.1); d) i costrutti con N Prep Nmc (§6.2.2): "scrittore da baci Perugina".

La tassonomia proposta è grossolana e parziale; non tutte le possibili ricorrenze di marchionimi utilizzati metaforicamente vi sono esemplificate. Lo studio proposto consente quantomeno di stabilire un punto di partenza per analisi successive.

\subsection{Marchionimi metaforici in funzione argomentale}

L'analisi degli usi metaforici si apre con una batteria di esempi in cui i nomi commerciali compaiono come nuclei di nessi nominali e non come modificatori. Le fattispecie sintattiche nelle quali si presentano sono varie, come vedremo, e vari i marchionimi che vi ricorrono. $\mathrm{Li}$ accomuna il fatto di avere valore antonomastico rispetto ad altri nomi commerciali dello stesso settore e di costituire quindi il prototipo di una categoria costruita attorno alla proprietà di volta in volta ritenuta rilevante.

\subsubsection{Usi non modificati: Det Nmc}

(1) "La categoria degli occhiali - spiega Donny Deutsch, il pubblicitario responsabile della campagna [di Luxottica] - non ha un leader. Anzi, la categoria, nella percezione del pubblico, non esiste neppure. Quando si dice bevande gassate, si pensa subito alla Coca-Cola o alla Pepsi. Quando si dice automobile, si pensa alla General Motors. Quando si dice occhiali, non c'è invece una Nike, o una Levi Strauss". (CORIS STAMPASupplementi)

Questo brano esemplifica il concetto di antonomasia (cf. Renzi 1988: 369): Coca-Cola e Pepsi, vi si afferma, rappresentano i marchi par excellence delle bibite gassate, General Motors delle automobili. Obiettivo del pubblicitario è colmare una lacuna in questo senso, facendo diventare Luxottica - si potrebbe dire - "la Nike o la Levi Strauss degli occhiali". 
I nomi commerciali Nike e Levi Strauss ricorrono come antonomasie delle 'marche più in vista del rispettivo settore merceologico', ovvero calzature sportive e jeans. Ricorrono preceduti da un articolo indeterminativo che, in questo tipo di struttura, è correlato all'interpretazione metaforica: una Nike è 'un'impresa o una marca come Nike'.

Valore simile hanno, nell'esempio che segue, gli articoli determinativi plurali:

(2) E la Roma? Non potrebbe comprare palloni prodotti senza sfruttamento? "È un problema di contratti, così ho messo in comunicazione l'associazione TransFair con la Diadora. Devono essere le grandi aziende, le Nike, le Adidas, a cambiare modo di produrre". (LaR)

Nike e Adidas sono menzionate qui come esempi di "grandi aziende" attive nel settore della produzione di materiale sportivo. In questo caso, ovviamente, il plurale non va correlato al valore di "scarpe" (§1.1.3), bensì riprende aziende e, come per una Nike (1), indica l'istituzione di una classe i cui rappresentanti prototipici sono Nike e Adidas: "le grandi aziende, quelle come Nike e Adidas".

Il brano seguente evidenzia un altro valore antonomastico di nomi commerciali:

(3) Sulla collina di Sant'Ilario, Levante di Genova, Beppe Grillo sorride: "La coppia Ripa Di Meana è inquietante, ma le Olimpiadi ad Atene per l'Italia sono una buona notizia. Vedevo le solite costruzioni, solite migliaia di miliardi al vento. Ricandidiamoci per quelle del 3010, quando Roma come Sydney si farà sponsorizzare da Greenpeace. Non si fa sport con le Coche Cola, le Nestlé, le McDonald's. E noi avevamo già l'accordo con la carne tritata, mucche pazze, capre marce. $[\ldots]$... (LaR)

Anche in (3) gli articoli determinativi al plurale segnalano l'uso antonomastico dei marchionimi: emblemi, in questo caso, di multinazionali, poco adatte, secondo il comico, a sponsorizzare eventi sportivi nel clima ecologizzante del nuovo millennio.

(4) "Nel nostro settore industriale possiamo paragonarci alla Coca Cola, ma non vediamo in giro nessun concorrente in grado di assumere il ruolo della Pepsi", si è vantato qualche giorno fa il vice direttore generale Hu Sanxing in un'intervista al settimanale Asia Week. (CORIS STAMPASupplementi) 
Qui il nome commerciale metaforico (Pepsi per 'antagonista alla pari di una marca di grande successo') è introdotto da un paragone esplicito. Pepsi è preceduto da articolo determinativo singolare: il riferimento è alla ditta o marca Pepsi; a essere metaforico, o antonomastico, è solo il valore di essa come eterna concorrente di Coca-Cola.

In (5) e (6) i nomi commerciali metaforici fungono da nome del predicato:

(5) Barbie non è affatto una invenzione recente, se mai è una (più o meno consapevole) riscoperta del passato. La bambola greca o romana era infatti anche lei una Barbie, sotto quasi tutti i punti di vista. (LaR)

(6) È spaventoso ripetere ciò che le dissi dieci anni fa: il corpo umano è una Ferrari, ma una Ferrari con i freni della 500. C'è un campo su cui non siamo progrediti e di cui non sappiamo nulla. Sto parlando dei tessuti trofo-meccanici, dei sistemi di sostegno. $(\mathrm{LaR})^{2}$

Nell'esempio (5), dell'oggetto cui si riferisce il nesso "La bambola greca o romana" si predica il possedere determinate caratteristiche in comune con la bambola Barbie: non tanto il biondo dei capelli o la bellezza artificiale, come nell'esempio citato in apertura del capitolo, quanto la conformazione, in particolare - come si legge più avanti nell'articolo il rappresentare "una ragazza dai quindici ai diciotto anni".

In (6) il marchionimo Ferrari è preso a modello di velocità e potenza (nell'articolo si parla di potenziamento delle prestazioni sportive), poi modificato con un altro termine di paragone tratto dal settore automobilistico (“con i freni della 500”; sulle modificazioni v. §6.1.2, infra).

Funge da nome del predicato anche il nome commerciale contenuto in (7):

(7) Non mi piace, non lo so fare, odio il telefono, sono una che ancora ama scrivere le lettere. Poi non voglio fare la Barbie, la bambola stereotipata, come ho visto fare alle star del sabato sera. (LaR)

Nella struttura fare la Barbie il marchionimo ha funzione predicativa (cf. La Fauci 2011: 203) ed entra nel costrutto definito HABITUs da La Fauci (2011: 75), nel quale la presenza dell'articolo determinativo

2 Cf. inoltre "Piperita [Patty] non è tipa da calendari. Anzi, è l'anti Barbie"(LaR). 
singolare è pertinente (La Fauci 2011: 204). Il nome commerciale vi compare come antonomasia vossianica (La Fauci 2011: 205n).

Un altro costrutto con predicato nominale è quello con diventare:

(8) Ed è una ragazza anche lei [Barbie], la bambola che nessuna bambina si sognerebbe di cullare o di imboccare perché suonerebbe come la profanazione di un piccolo sogno: quello di diventare davvero una Barbie, in un futuro prossimo di fianchi stretti, gambe tornite, lunghissime chiome bionde, incredibili piedini dal tallone rialzato, fatti per il tacco a spillo. (LaR)

In questo esempio il valore peculiare da attribuire all'uso metaforico di Barbie, relativo a caratteristiche estetiche, è esplicitato nel prosieguo del brano. Si osserva dunque che le caratteristiche messe in luce dagli usi metaforici sono di volta in volta diverse, a riprova del fatto che l'interpretazione dei nomi propri con valore di metafora è strettamente legata al contesto di ricorrenza (cf. Wee 2006: 358).

Ricorrono pure predicati nominali utilizzati in comparazioni e introdotti da come (cf. Vande Casteele 2014: 664), che indicano similitudine tra il soggetto e il marchionimo in funzione, ancora una volta, predicativa:

(9) Anche se il blocco del traffico è previsto dalla legge è come un'aspirina: fa calare la febbre, ma non aggredisce le cause dell'inquinamento. (CoLFIS, quotidiano)

In questo caso la similitudine prosegue con una metafora isotopica ( $f a$ calare la febbre).

(10) Sia detto per inciso ormai di Brunello 90, quello andato in enoteca lo scorso anno, non ce n'è più: tre milioni di bottiglie assorbite come se fossero una goccia e il mercato uno Scottex. (LaR)

(11) Ma non importa, se il segretario di Stato americano non c'è: alle ragazzine con la coda affacciate dall'alto del castello Odescalchi, ai fotografi venuti a grappoli, interessa molto di più l'inarrivabile incedere di John John Kennedy, il Kennedy più fedele e docile del mondo, ancora e sempre al braccio di quella moglie bionda e sottile come una barbie, tubino nero, crocchia, pelle candida perché gli americani ricchi non si abbronzano nemmeno d'agosto, è volgare e fa male alla pelle. (LaR) 
(12) Simili a Barbie di gran lusso, le amabili signore vestite da Ungaro pongono un solo problema: quando avanzano, allegre e sprezzanti, in gruppo, confidandosi evidentemente porcherie di gran classe, sembrano non aver bisogno di niente se non di stare insieme tra loro. (LaR)

(13) I medici lo hanno ammesso: il cuore di Bartali batte sano e lento come il motore di una vecchia Guzzi. (LaR)

(14) Il Mediterraneo appare sempre di più come un'enorme Jacuzzi. Temperature da acque termali, a cui mancano solo le bolle $[\ldots] .(\mathrm{LaR})^{3}$

Ecco, infine, un esempio diverso di uso argomentale del marchionimo:

(15) Benvenute, signore, nel caravanserraglio dell'Italia Tv. C'è posto per tutte: per l'ilare Wendy Windham e l'elastica Heather Parisi, per la Barbie Carol Alt e la frizzante Natalia Estrada [...]. (LaR)

Nel costrutto la Barbie Carol Alt il nome commerciale si può interpretare come una sorta di apposizione interna, per cui esso avrebbe, appunto, funzione argomentale ("la Barbie [di nome] Carol Alt", come "il compositore Verdi"). Da un altro punto di vista, e tenendo conto dei costrutti che precedono e seguono il nesso in questione, tale marchionimo è però funzionalmente equivalente a un aggettivo, quindi a una modificazione predicativa (v. §6.2.1).

\subsubsection{Usi modificati: Det Nmc Mod}

I nomi commerciali - e i nomi propri in generale - utilizzati metaforicamente ricorrono spesso accompagnati da modificatori (cf. Jonasson 1991: 66):

(1) I marchi delle industrie più note camminano con successo sulle nevi di tutto il mondo. Chi non conosce, infatti, la Diadora (Caerano San Marco e 400 dipendenti), la Nordica, chiamata in zona la "Fiat" della scarpa da sci (Montebelluna e 700 dipendenti), la Tecnica (Nervesa), Caber del gruppo Rossignol, Lotto, Brixia di Asolo oppure Dolomite? (LaR)

3 Sulle modificazioni del marchionimo usato metaforicamente (esempi 12-14) v. $\S 6.1 .2$. 
In questa struttura - Det Nmc di N-il nome commerciale Fiat ricorre con valore antonomastico ed è modificato da un complemento, della scarpa da sci, che riferisce le peculiarità pertinenti del marchionimo (in questo caso il fatto di essere una grande impresa, conosciuta e di successo) a un diverso settore produttivo: non veicoli ma, appunto, scarponi da sci. Jonasson (1991: 77-78) osserva che questo genere di complementi, che definisce "classificanti", ricorre perlopiù in combinazione con un articolo deteterminativo che precede il nome proprio, come in (1).

Si ritrovano modificatori implicanti un riferimento spaziale (cf. Wee 2006: 365-366):

(2) Più volte annunciata, finalmente Imelda [Marcos] compare. Per questa Barbie asiatica il tempo invece esiste. È alta sui tacchi dorati delle sue scarpe nere, i capelli lucidi tirati sulla testa, il vestito blu con i bottoni davanti è ampio abbastanza da difendere la pinguedine ma le maniche corte scoprono braccia carnose e non più sode. (LaR)

(3) Ma i sex symbol nostrani arrancano in classifica, il cuore dei ragazzi batte per le patinate Barbie d'oltreoceano. Alla domanda "chi vorresti come compagna di studi" le ragazze di Beverly Hills si rivelano come le perfette school girls. (LaR)

Mentre Barbie allude a un aspetto esterno o atteggiamento, secondo il modulo visto più volte in queste pagine, i modificatori asiatica e d'oltreoceano presentano caratteristiche del destinatario (target: cf. Wee 2006: 357) del marchionimo metaforico: Imelda Marcos è asiatica; le ragazze che affascinano i giovani italiani sono americane.

I modificatori o complementi, osserva Jonasson (1991: 77), si riferiscono a quello che la studiosa denomina "topique", ossia l'entità di cui si predica il nome proprio metaforico, e il più delle volte si oppongono a ciò che si sa del referente originale. Al proposito, però, va fatta un'osservazione. In realtà importa poco che le Barbie che fanno da fonte (source; Wee 2006: 357) per l'uso metaforico di (3) siano effettivamente "d'oltreoceano". Come nota Jonasson (1991: 77) nel caso dell'esempio "il se laisse draguer dans les coulisses par une Lolita de douze ans" (cit. a p. 68) - la Lolita originale di Nabokov ha davvero dodici anni -, con nomi propri il cui valore metaforico si è lessicalizzato, fissandosi nel tempo su determinate caratteristiche della fonte 
(Lolita: ragazzina seducente, sessualmente precoce; Barbie: donna dalla bellezza artefatta), avviene che altre proprietà siano obliterate.

Strutture simili, con modificatore che allude (indirettamente) alla provenienza geografica, ricorrono pure in riferimento ad altre bambole simili a Barbie:

(4) Le linee dei prodotti più noti sono, oltre alla bambola Tanya (una Barbie mediterranea) e alla linea Power Rangers, i videogiochi Sega, la bambola Cicciobello, alcuni pupazzi di licenza giapponese. (LaR)

(5) [Tanya Miss Italia] È una sorta di Barbie autarchica dai lunghissimi capelli ramati con dotazione di scettro dorato e coroncina tempestata di rubini e un grande mazzo di rose scarlatte. (LaR)

Tanto autarchica quanto mediterranea, combinati con Barbie e con determinatori di tipo indeterminativo (una, una sorta di), modificano il marchionimo menzionato come elemento prototipico della classe "bambole dalle fattezze di donna".

Le modificazioni non sono soltanto spaziali:

(6) Ecco qui la Ulrica di Diane Curry, mediocrissima vocalmente ma presentata come una Barbie d'angiporto, coi lunghi capelli di fil di ferro, ottenuti con chili di lacca o con una gita in motocicletta. (LaR)

In (6) d'angiporto è complemento caratterizzante (secondo la distinzione di Jonasson 1991: 77-78; la marca compare infatti con articolo indeterminativo).

(7) Allora bisogna chiederlo a lui: Frankie, come mai esiste questo meraviglioso sistema metrico musicale che è il rap, e in Italia, a parte qualche genialata tua e di pochi altri, i testi fanno pena? Non so se mi sono spiegato: immaginate una nutella di sillabe che si può stendere qui a strati sottili e lì a grumi densi. (CORIS STAMPASupplementi)

(8) Un altro cult di Cesarone è la formazione. Pur di non darla ai cronisti, non la dà a se stesso. Si crogiola nella nutella della pretattica: "Zola? Benino. Del Piero? Bene. Per favore: non voglio giochini da terza B". (CORIS STAMPAQuotidiani)

In questi brani il marchionimo nutella, in funzione argomentale, è utilizzato con il valore predicativo di 'crema densa, spalmabile'. Entro il costrutto esso funge in realtà da determinatore complesso di forma 
nominale, come nei casi di "una serie di spari" o "una valanga di applausi” (La Fauci 2009: 146-147).

\subsection{Marchionimi metaforici come modificatori}

I nomi commerciali possono manifestare valore metaforico anche quando fungono da modificatori. In un articolo che tratta di vip e parrucche si legge:

(1) Ciuffetti extra usa Lorena Bianchetti con un curioso effetto spaghetto che qualcuno ha ribattezzato taglio Voiello. (CORIS MON2005_07)

Voiello ricorre qui come allusione ironica allo spaghetto che ricorre poco prima.

Una differenza rispetto agli esempi del $§ 6.1 .2$ consiste nella combinazione "evocativa" (cf. Zardo 2007: 266) di nome comune e marchionimo, senza che esso costituisca l'esemplare per antonomasia di una classe o che sia fatto riferimento a caratteristiche specifiche che lo distinguono da altri. Nell'es. (1) Barilla o altri marchionimi relativi al settore della pasta avrebbero potuto svolgere la medesima funzione di Voiello.

Il marchionimo compare come modificatore negli esempi del $\S 6.2 .1$ e introdotto da preposizione nei brani del $\S 6.2 .2$.

\subsubsection{Modificazione semplice: N Nmc}

(1) Li hanno già definiti $i$ lavoratori kleenex: ingaggiati il più delle volte con contratti precari (da co.co.co. e co.co.pro.), sono considerati i dipendenti usa e getta per eccellenza degli Anni Duemila. (CORIS MON2005_07)

Il nome commerciale kleenex, in funzione modificativa, è usato con il significato predicativo, piuttosto stabile, di "usa e getta" (cf. Vande Casteele 2014: 663-664). 
Il nesso omino Michelin (che allude a Bibendum), con marchionimo in funzione predicativa, ricorre in riferimento a persone tondeggianti o "gonfiate":

(2) E all'improvviso l'omino Michelin diventò un aquilone, volò leggero. Vialli il culturista, Vialli il convalescente a vita, Vialli il gonfiato, il montato, il finito. (CoLFIS, quotidiano)

Ben più frequente è il modificatore (Baci) Perugina:

(3) Una folla immane, soprattutto ieri sera, inchiodata alla voce angelicata di Mango e al femminismo Perugina di Fiorella Mannoia: il famoso popolo italiano ieri sera si è riunito in mondo visione ad anime ugualmente musicali e sentimentali turche e marocchine, polacche e cubane. (LaR)

(4) Io ho l'impressione che in questa squadra molti giocatori abbiano imparato a memoria quattro-cinque frasette tipo Baci Perugina, frasette in cui umiltà fa sempre rima con intensità e volere quasi mai con potere. (LaR)

(5) E così, nel guardaroba dell'uomo, stilisti e industriali sono pronti a far entrare di tutto: dal giaccone termoriscaldato di Exté, ai cappotti in stile direttorio abbinati a pantaloni che portano messaggi affettuosi cuciti nella patta. Un esempio? "Bravo", oppure "Fonte di gioia infinita" e altre amenità, genere baci Perugina. $(\mathrm{LaR})$

Il marchionimo modifica predicativamente nessi nominali diversi (eventualmente con l'inserimento dei classificatori tipo e genere, come in $4 \mathrm{e}$ 5). Il suo valore predicativo va ricercato non nel prodotto ma nei fogliettini che lo avvolgono e nei testi che contengono. L'ipotetica voce di un dizionario degli usi figurati di marchionimi potrebbe recare alla voce Baci Perugina: "In riferimento ai temi e allo stile delle massime stampate sui cartigli che avvolgono i cioccolatini omonimi, allude, secondo i casi, a una scrittura aforistica di bassa lega, di argomento filosofico o amoroso, o alla celebrazione banaleggiante dei buoni sentimenti". L'esasperazione dei tratti più caratteristici delle citazioni dà all'impiego metaforico una sfumatura solitamente spregiativa.

Gli esempi, nei dati, si sprecano. Ecco alcuni casi, questa volta di strutture in cui il nome commerciale ha funzione argomentale e non di modificatore: 
(6) Si apprende che il cosiddetto gigolò Helg Sgarbi, condannato a Monaco di Baviera (sei anni) per avere estorto denaro alle sue ricche e deboli amanti, ama sua moglie "più di ieri meno di domani", formulazione così patetica da non trovare più posto neanche sui cartigli dei Baci Perugina. (LaR online $\left.{ }^{4}\right)$

(7) [Stefano Bonaga] lo ha raccontato, a modo suo, scrivendo un saggio Sulla disperazione d'amore, Feltrinelli Universale Economica. 119 massime, scritte in una prosa a metà tra filosofia e baci Perugina. Tutte amarissime, tristissime, senza speranza. (LaR)

(8) Fioriscono sulla bocca del nostro Presidente tutti i luoghi comuni che accadeva, una volta, di ascoltare in treno o sulla spiaggia sotto un ombrellone. Da un po' di tempo queste affermazioni non avevano più diritto di cittadinanza. $\mathrm{Ci}$ voleva il nostro Presidente per riproporle, con la dignità delle sentenze che avvolgono i Baci Perugina. Eccone alcune: "Tratto significativo della donna è la sua capacità di donarsi, la sua vocazione specifica è quella di moglie, madre, compagna”. (LaR)

\section{Ma torniamo agli usi predicativi.}

(9) Ai mondiali, il 10 luglio del basket è stata una copia Rank Xerox del 18 giugno del calcio. Due Italie diverse: nella forma, nelle avversarie, ma eguali nella sostanza, nell'essere una navicella spaziale che vagava nel vuoto. (LaR)

Il brano (9) è un esempio di impiego evocativo del marchionimo a fini espressivi, senza peraltro che la predicazione faccia riferimento a caratteristiche particolari del referente (come nel §6.1): è in gioco solamente il significato, generico, di 'fotocopia'.

(10) In questo quadro non si esclude il lancio di una Opa sul restante 25 per cento di capitale Ausimont del quale il 19,5 si trova nei portafogli di investitori istituzionali. Successivamente la Montedison Usa potrebbe fondersi con l'Ausimont senza eccessivi oneri fiscali e venire in possesso dell'intera liquidità. Un caso Buitoni insomma con marchio americano. (LaR)

In (10), invece, un episodio che ha coinvolto l'azienda Buitoni assurge a modello per avvenimenti simili, in maniera antonomastica (cf. §6.1). Del referente è messa in rilievo una faccetta particolare: il coinvolgimento, appunto, in un caso.

$4 \quad<$ ricerca.repubblica.it/repubblica/archivio/repubblica/2009/03/11/amaca.html ?ref $=$ search $>$ (9.6.2015). 
Torna poi l'esempio, già noto, di Barbie, stavolta in funzione predicativa:

(11) Ma Halloween è anche uno strumento tutto femminile per ribellarsi al modello Barbie: "Sono tante le bambine che stanche di treccine perfette chiedono di essere trasformate in streghe orrende". (LaR)

(12) Arianna David, reginetta modello Barbie che di italico non ha nulla, può dormire sonni tranquilli: l'aver fatto la comparsa in tivù al "Gioco dell'oca" e la valletta di fila nello show "Acqua calda" non mette a repentaglio la sua coroncina di latta [...]. (LaR)

(13) [...] perché [...] signore frastornate da momenti grami, lavorativi o affettivi, dovrebbero fuggire a gambe levate da mutande, slip, reggipetti, baby doll, sottovesti, che pare saranno la base del nostro abbigliamento per la prossima estate, o dalle parrucche, possibilmente platinate stile Barbie, o Sylva Koscina ai suoi bei tempi $[\ldots]$ ? (LaR)

In combinazione con stile e modello, il marchionimo Barbie ricorre metaforicamente per caratterizzare gli aspetti estetici già ricordati.

Barbie compare inoltre come secondo elemento (modificatore) in nomi di colore:

(14) La cosa peggiore di Pavel Nedved sono quei capelli biondo Barbie, vezzo civettuolo di un uomo senza vizi, che però sventolano quasi come una bandiera quando lui affetta i campi di calcio [...]. (CORIS MON2001_04)

(15) "Per far risaltare l'abbronzatura usate le nuance arancio-corallo [...]" dice Mario De Luigi, truccatore e creatore del make up Pupa. "La sera concedetevi l'effetto maculato su base bronzo ramato, oppure una bocca bicolore nei toni del carminio lucente e del rosa Barbie". (CORIS STAMPAPeriodici)

\section{Si conclude con un altro caso di marchionimo in funzione evocativa e metonimica:}

(16) Su mille intervistati, tutti di età superiore ai 18 anni, il 64,6 per cento invoca la chiusura anticipata delle discoteche, e addirittura l'86,4 per cento chiede l'immediata entrata in funzione del palloncino anti-alcol. Fine degli anni Timberland, tramonto di ogni sballo, ballo, eccesso? (LaR) 
Il riferimento è qui al marchio delle scarpe che hanno contrassegnato un'epoca e, per estensione (metonimica), passa a caratterizzare il clima culturale di quegli anni.

\subsubsection{Con preposizione: $\mathrm{N}$ da $\mathrm{Nmc}, \mathrm{N}$ a(Det) $\mathrm{Nmc}$}

(1) Un po' di Dolce Vita, una spruzzata di storia, uno slogan che sa di eternità, "la città senza tempo", tre concetti da baci Perugina "fratellanza, dialogo, pace", e il viaggio sottobraccio alle Olimpiadi andrà avanti. (LaR)

Così si legge in un articolo che commenta la candidatura di Roma ai Giochi olimpici del 2004. Il valore predicativo del nome commerciale baci Perugina, preceduto dalla preposizione $d a$, è grossomodo quello individuato per gli esempi dei paragrafi precedenti.

Nei dati la maggior parte degli esempi della struttura $N d a N m c$, già ricordata nel capitolo sui nessi preposizionali (§3.3), è costruita proprio con Baci Perugina:

(2) Tra i detrattori [della lettera di Epicuro a Meneceo] figura anche Alberto Bevilacqua: "È un'opera dalla limpidezza involontariamente umoristica", dice. "Mi sembrano frasi fatte apposta per incartare i cioccolatini degli dei. Si dicono cose di un'ovvietà assoluta". "Scrittore da baci Perugina sarà lui, non Epicuro", è la replica secca di Baraghini. (LaR)

Il nome commerciale baci Perugina riprende, nell'affermazione di Baraghini, l'accenno implicito dello scrittore Bevilacqua sullo stile del testo di Epicuro: "frasi fatte apposta per incartare i cioccolatini degli dei".

Anche una "filosofia" o una "retorica" possono essere "da baci Perugina":

(3) Gianfranco Funari - Filosofia da Baci Perugina o da siparietti di Dapporto, sorriso da imbonitore, gesticolazione da portiere di Jolly Hotel, più Upim che Rinascente, conflittualità da trattoria. Voto $0(\mathrm{LaR})$

(4) Il cronista non può fare a meno di sottolineare l'omogeneità dei testi, la loro scarsa originalità, la tendenza ad un certo infantilismo e ad una retorica da Baci Perugina post-sessantottini. Gli autori dei messaggi sembrano tutti prendersi per poeti, sparano immagini idilliche e non sembrano minimamente sfiorati dal senso del ridicolo. (LaR) 
In (5) il nesso $d a+$ nome commerciale funge da predicazione nominale:

(5) L'amore è il suo tema ricorrente. L'accusano di essere da "Baci Perugina"? «Nei Baci Perugina ci sono anche le poesie della Dickinson, che amo, o Proust. Se le cose che scrivo fossero così scontate, vivremmo meglio. [...]». $\left(\mathrm{LaR}^{5}\right)$

La formulazione da Baci Perugina pare aver acquisito un tale grado di fissità da poter ricorrere, appunto, come predicazione in un costrutto con copula.

Esempi con la preposizione $a$ sono più rari:

(6) "Ma la novità è tra i giovanissimi che indossano colori che vanno via con una spazzolata, tinture che dopo uno shampoo ricominci, capelli sereno variabile, perché tutto è rapido e sostituibile, in una vita alla Barbie dove ogni giorno si cambia”. (LaR)

(7) Mi viene da confrontare le facce da bebè al Plasmon dei calciatori (che non giocano più di cinquanta minuti effettivi su novanta) con queste facce tirate e scavate da minatori [dei ciclisti]. (CoLFIS, quotidiano) ${ }^{6}$

In (6) una vita alla Barbie è simile ai nessi "stile/modello Barbie" visti in precedenza.

Nell'esempio (7) il particolare costrutto le facce da bebè al Plasmon è modellato probabilmente su "biscotti al Plasmon", che ricorre in usi referenziali. ${ }^{7}$ Il riferimento metaforico sposta l'attenzione da un prodotto per bambini ai suoi fruitori, i bebè dell'esempio.

$5 \quad<$ ricerca.repubblica.it/repubblica/archivio/repubblica/2015/02/08/massimo-bi sotti-favole-damore-baciate-dai-socialMilano10.html?ref=search $>(9.6 .2015)$.

6 Il brano ricorre, con minime variazioni, in un articolo dello stesso autore, Luca Goldoni, pubblicato sul Corriere della Sera il 16.2.2004 (<archiviostorico. corriere.it/2004/febbraio/16/SOLE_NERO_CHE_ACCECO_CAMPIONE_ co_9_040216006.shtml>, 12.6.2015). Curiosamente lo stesso paragone tra ciclisti e calciatori compare anche in un articolo di Donatella Tiraboschi su L'Eco di Bergamo, 17.5.2009 (<webstorage.mediaon.it/media/attach/2009/10/ orrillo3.pdf>, 12.6.2015): "Le smorfie dei ciclisti non sono come quelle dei calciatori, con le loro facce da bebè al plasmon".

7 "Per fare la campagna dei Biscotti al Plasmon Pirella lesse interi trattati di puericultura” (CORIS MON2001_04). 


\section{Conclusioni}

Si riassumono alcuni aspetti emersi dall'analisi dei marchionimi metaforici, la quale, si ricorda, ha carattere esemplare e non esaustivo.

Va osservato innanzitutto che la natura - si dirà - intrinsecamente predicativa dei nomi commerciali, funzionalmente considerabili come aggettivi, si riflette nella possibilità che essi ricorrano come modificatori a valore metaforico; una possibilità non contemplata dalle trattazioni relative agli usi metaforici dei nomi propri (cf. Jonasson 1991, citata nell'introduzione). Si pensi a "la Barbie Carol Alt", "lavoratori kleenex" o "femminismo Perugina".

L'etichetta di "metaforico" si applica a diversi tipi di usi traslati. Da una parte vi sono gli usi antonomastici ("una Nike" per 'un'azienda leader', come Nike nel settore sportivo; "le Nestlé" per 'multinazionali'), nei quali il marchionimo ricorre come esemplare prototipico di una determinata categoria, eventualmente accompagnato da un modificatore che specifica una caratteristica del target ("la Fiat della scarpa da sci"). Dall'altra parte vi sono usi, che Zardo definisce "allusivi" ed "evocativi", nei quali il nome commerciale non è (necessariamente) menzionato per il suo valore di esemplare prototipico: "una copia Rank Xerox", "taglio Voiello".

Emergono nomi commerciali il cui valore metaforico si è fissato nell'uso: Barbie è, per così dire, un' antonomasia in stadio avanzato che ricorre financo nei dizionari, ${ }^{8}$ mentre l'uso metaforico di Baci Perugina non ha avuto (sinora) l'onore di essere registrato dai lessicografi, probabilmente perché, oltre a trattarsi di un nome composto, esso ricorre soprattutto in funzione predicativa, mentre per Barbie si registrano perlopiù usi argomentali.

I marchionimi con valore metaforico compaiono spesso in strutture diverse da quelle indicate da Jonasson (1991) per i nomi propri. L'argomento sarebbe pertanto da approfondire nei suoi aspetti combinatorî oltre che semantici.

8 Cf. Treccani s.v. barbie 2 : "Per lo più in senso spreg.[iativo], ragazza o donna truccata e dall'acconciatura molto curata ma di bellezza un po' artefatta, che ricorda, nell'aspetto, tale bambola" (<www.treccani.it/vocabolario/barbie/>, 9.6.2015). Antonomasie pienamente lessicalizzate (assimilabili ai nomi comuni) sono per es. walkman o post-it (cf. Parte I, §3.3 e §4.2). 


\section{Dagli Avirex ai Miss Sixty in dodici anni. Marchionimi e narrativa: analisi di Federico Moccia, Tre metri sopra il cielo}

\section{Introduzione}

I marchionimi sono l'emblema di Tre metri sopra il cielo, romanzo rosa di Federico Moccia edito nel 1992 e ripubblicato, con modifiche, nel 2004. ${ }^{1}$ Un emblema, ossia un elemento strutturale che caratterizza l'opera e ne riassume alcuni tratti essenziali.

Non si tratta solo di una questione numerica. È vero che il romanzo mocciano è costellato di brand (nell'edizione del 1992 si contano quasi quattrocento token, in media uno per pagina), ${ }^{2}$ ma questa non è certo un'eccezione nel panorama narrativo recente (cf. Sini 2014). L'inserimento di nomi commerciali in romanzi e racconti, infatti, è usualmente sfruttato per pennellare con tratti di realismo la scenografia urbana davanti alla quale si svolgono le vicende narrate, creando una "Namenlandschaft" (Gutschmidt 1982: 23). ${ }^{3}$ I marchionimi hanno pure la funzione di delineare identità, statuto sociale e gusti dei personaggi (cf. Sini 2014: 2317-2318; Gutschmidt 1982: 24). Nei romanzi, inoltre, i nomi commerciali possono caricarsi di valenze metaforiche o allusive

1 Il cap. 7 riprende e rielabora parzialmente Janner (2012b), cui si rimanda per ulteriori aspetti dell'analisi.

2 Oltre che per la loro presenza ingombrante (Matt 2007: 248 osserva che Moccia "infarcisce i suoi romanzi di passaggi molto simili a spot pubblicitari, soprattutto relativi a capi di vestiario"), nel romanzo i marchionimi saltano all'occhio, e in certo modo infastidiscono il lettore, perché menzionati in maniera gratuita, fondamentalmente inessenziale alla trama (low plot integration; Olsen \& Lanseng 2012 osservano una correlazione, in questi casi, con una percezione meno positiva della marca).

3 Si vedano gli studi relativi al linguistic landscape (cf. Tufi \& Blackwood 2010 per un'analisi dei nomi di marca che appaiono nel paesaggio linguistico). 
(cf. Zardo 1997: 33) quando siano utilizzati per le caratteristiche connotative che hanno acquisito nel tempo (su questo v. il cap. 6).

La questione, come si sa, è economica oltreché strettamente letteraria: il brand placement, vale a dire l'inserimento (a pagamento) di marchionimi in opere di intrattenimento, dai telefilm ai romanzi, è diventato l'ultima frontiera della pubblicità e un attraente campo d'investimento per le aziende, benché controverso dal punto di vista etico (cf. Olsen \& Lanseng 2012). Ma di ciò, in questa sede, non si tratterà oltre. $^{4}$

I nomi commerciali sono un emblema di Tre metri sopra il cielo da un profilo linguistico, strutturale e funzionale; sia internamente al sistema dell'opera narrativa, quanto alla tipologia sintattica degli usi, sia nella dimensione diacronica del passaggio dalla prima alla seconda versione pubblicata, laddove uno dei cambiamenti principali è proprio la sostituzione di alcuni marchionimi con altri. E, come si vedrà, i due aspetti sono correlati.

In questo capitolo l'analisi sintattica, condotta sin qui su testi precipuamente giornalistici, sarà applicata a un altro genere testuale, la narrativa, e all'insieme sistematico di un'opera letteraria. Lo studio di un testo circoscritto permette di eseguire uno spoglio completo dei costrutti contenenti nomi commerciali, sì da individuare costanti e variazioni, tratti caratteristici delle ricorrenze e loro valore sistemico. L'analisi si colloca nel contesto più ampio degli studi di onomastica letteraria, ${ }^{5}$ compiuti però in prospettiva morfosintattica, in linea con il resto del lavoro. ${ }^{6}$

$4 \quad$ Nel caso di Tre metri sopra il cielo tenderei a escludere un influsso commerciale sull'ingente uso di marchionimi: non è chiaro per quale motivo le aziende avrebbero voluto investire in un giovane autore esordiente e sconosciuto, com'era Moccia nel 1992. Alcuni nomi di marca, peraltro, sono riportati con grafie scorrette.

5 Cf. Nicolaisen (1995) per un'introduzione relativa alla letteratura inglese e le pubblicazioni della rivista il Nome nel testo. Per l'italiano v. inoltre i lavori di Bruno Porcelli e Maria Giovanna Arcamone.

6 Ci si concentra su aspetti (micro)sintattici più che morfologici; per es. non si approfondirà il fatto che alcuni marchionimi ricorrono modificati morfologicamente (Vespone, Vespine; Kawa per Kawasaki). 


\subsection{Usi argomentali e predicativi. Analisi linguistica}

In un romanzo attento alla moda, che differenzia e qualifica i propri personaggi anche in base alle marche degli oggetti che essi possiedono o non possiedono (cf. Janner 2012b: 221-222), non stupisce che i referenti dei marchionimi menzionati siano individuabili più tra i prodotti che non tra le imprese. Ecco una prima, importante differenza rispetto ai testi giornalistici che hanno costituito sin qui la base dell'analisi. Nel libro non ricorrono strutture quali targato Nmc o alla Nmc dicono, interpretabili in riferimento a nomi d'impresa. Sono del tutto assenti, peraltro, gli usi avverbiali delineati nel $\S 4.4$.

Così si apre la narrazione, introducendo il protagonista maschile, Step:

Capelli corti, quasi a spazzola, sfumatura dietro il collo alta come quella di un marine, un giubbotto Levi's scuro.

Il colletto tirato su, una Marlboro in bocca, $i$ Ray-Ban agli occhi. Ha un'aria da duro, anche se non ne ha bisogno. [...]

Alcune macchine in fondo al cavalcavia si sono fermate minacciose al semaforo. Eccole lì, in riga come in una gara, se non fosse per la loro diversità. Una Cinquecento, una New Beatle [sic], una Micra, una macchina americana non meglio identificata, una vecchia Punto.

In una Mercedes 200, un esile dito dalle piccole unghie mangiucchiate dà una lieve spinta a un cd. Dalle casse Pioneer laterali la voce di un gruppo rock prende improvvisamente vita. (Moccia 2004: 9)

Le ricorrenze di marchionimi comprendono tanto usi argomentali ("una Marlboro in bocca") quanto predicativi ("un giubbotto Levi's scuro"). Nel romanzo gli usi argomentali prevalgono decisamente, nella misura di quattro casi a uno, sugli altri (cf. Janner 2012b: 224).

Capita che lo stesso referente - la cintura usata durante una gara di moto - sia denominato attraverso tre tipi diversi di strutture, tra loro equivalenti:

"Se ti metti la cinta di Camomilla ti scelgono loro e ti portano dietro, dai prendi la mia, pensa che forza, fai la 'camomilla'..." (Moccia 2004: 133)

In quel momento arriva Maddalena e sale dietro di lui. Si mette al contrario come dice il regolamento e passa la sua cinta Camomilla. (Moccia 2004: 139) 
Oddio, pensa Babi. [...] Questa cinta di Camomilla che mi stringe in vita. Io la Camomilla non me le [sic] sono mai messa, neanche quando era di moda. Dev'essere una punizione. (Moccia 2004: 143)

Nei primi due casi il marchionimo svolge funzione predicativa, eventualmente entro un nesso preposizionale (struttura $N(\mathrm{di}) \mathrm{Nmc}$ ). ${ }^{7} \mathrm{Nell}$ 'ultimo caso, invece, il nome commerciale ha funzione argomentale ed è preceduto da articolo. ${ }^{8}$

Tra gli usi argomentali e quelli predicativi vi è una differenza fondamentale: il grado di opacità del costrutto. Il nesso la cinta (di) Camomilla è meno opaco di la Camomilla in quanto contiene un riferimento esplicito all'oggetto in questione. Soltanto una conoscenza condivisa - in questo caso, costruita nel corso dell'episodio: l'uso argomentale appare dopo sette ricorrenze predicative - fa sì che il riferimento di la Camomilla sia univoco e che con tale nesso non ci si riferisca a una maglietta o una borsa della stessa marca. Gli usi argomentali sistematicamente determinati (§1.1.3), in questo assimilabili ai nomi propri, chiamano in causa conoscenze di tipo enciclopedico, più difficili da acquisire e più facili da perdere rispetto a quelle lessicografiche.

Come anticipato, la maggior parte delle ricorrenze di nomi commerciali nel romanzo di Moccia è da ascrivere al tipo sintattico (Det) Nmc. Quali caratteristiche presentano allora i costrutti - marcati - di tipo $N N m c$ ?

"Be', ora basta," e apre un libro che tira fuori dalla sua borsa Nike in pelle, "devo pensare al mio vero e unico problema." (Moccia 2004: 11)

"Nella nostra Hit Sudate al numero due c'è Hook. Come potete ben vedere, la splendida maglietta Ralph Lauren ha cambiato colore. Ora direi che è di un verde scolorito, o meglio, verde fradicio." (Moccia 2004: 34)

Pallina, con una tuta Adidas felpata bluette proprio come l'elastico che le stringe il ciuffo, corre quasi rimbalzando sulle Nike chiare. (Moccia 2004: 109)

7 Qui la preposizione non è articolata. Sulla questione si veda supra, §3.2.2.

8 Camomilla ricorre inoltre, con valore metonimico, per indicare le ragazze che portano questa cintura durante le gare di moto, ed è in questo caso declinabile al plurale: "I ragazzi sulle moto sono tutti rivolti in avanti, pronti a partire. Le camomille sono sedute dietro, girate." (Moccia 2004: 139). 
I casi di uso predicativo, lo si nota in questi brani, riguardano accostamenti di nesso nominale (relativo a un prodotto) e nome di marca che non sono sufficientemente ridondanti - nel sistema linguistico o, meglio, nel sistema del testo - per ricorrere con il semplice marchionimo. ${ }^{9}$ Si prenda l'ultimo esempio: accanto a "una tuta Adidas", con menzione del nome comune dell'oggetto, ricorre l'espressione "sulle Nike chiare", in rapporto alla quale il rimando a "scarpe" è implicito.

Quanto alla relazione con il predicato verbale, in alcuni casi i nomi commerciali si combinano a predicati che legittimano soggetti [+ umani] (cf. §4.1):

Fiore, il portiere, alza la sbarra. La Mercedes aspetta, come ogni sera, il lento salire di quel lungo ferro a strisce rosse. (Moccia 2004: 16)

Una sorta di personificazione del marchionimo avviene pure nel caso seguente:

Lui scende dai gradini di marmo, si sistema i suoi 501 e poi sale sull'Honda blu vf 750 Custom. Come per incanto si ritrova fra le macchine. La sua Adidas destra cambia le marce, richiama o lascia andare il motore, che, potente, lo spinge come un'onda nel traffico. (Moccia 2004: 9)

Non è il piede di Step a cambiare le marce: è la scarpa, anzi "la sua Adidas", in una mercificazione del corpo umano che fa da contraltare all'umanizzazione dei prodotti.

In Tre metri sopra il cielo tutte le ricorrenze di marchionimi, senza eccezione, sono strettamente referenziali. Laddove ricorrono, $\mathrm{i}$ nomi commerciali sostituiscono o specificano, modificandoli predicativamente, altri nomi relativi a prodotti: automobili, motorini, jeans, magliette, budini al cioccolato e gomme da masticare, disegnando un panorama scritto, denominato, da decifrare in relazione al sistema di valori proposto nel romanzo.

Che nella narrativa i nomi commerciali possano essere utilizzati in modo diverso, in particolare con funzione allusiva o ironica (cf. Zardo 1997: 32-36), è illustrato da alcuni brani di Jack Frusciante è

9 L'unico esempio in cui il nesso modificato non si riferisce a un prodotto è "una signorina Telecom" (Moccia 2004: 131). 
uscito dal gruppo, opera d'esordio di Enrico Brizzi pressoché coeva alla prima edizione del romanzo mocciano:

[Martino e Alex] Si frequentavano da non troppo tempo col patto tacito che lui non gli avrebbe fatto conoscere nessuno dei suoi amici house e Alex, in cambio, non avrebbe mai tentato d'introdurlo nel mondo Fender del punk parrocchiale. (Brizzi 1994: 29)

Adesso in ogni caso, i capitali di Marx e i diari boliviani del comandante Che, la madre di Martino li teneva ben allineati nella libreria di questa villa sui colli in cui viveva col figlio, mantenuta dall'ex marito, perfettamente a suo agio nei panni di Signora in clark's che impiega il suo Passato Proletario solo per fornire qualche brivido esotico ai nuovi amici massonimprenditorialrot-taryani. (Brizzi 1994: 40)

La realtà è che mi trovo costretto a sacrificare il me diciassettenne felice di oggi pomeriggio a un eventuale me stesso calvo e sovrappeso, cinquantenne soddisfatto, che apre la porta del garage col comando a distanza e dentro c'ha una bella macchina, una moglie che probabilmente gli fa le corna col commercialista e due figli gemelli con i capelli a caschetto identici in tutto ai bambini nazisti della kinders. (Brizzi 1994: 42)

Più che il riferimento a un prodotto o un marchio, in questi casi è cruciale l'allusione a fenomeni culturali, persino agli imballaggi dei prodotti stessi: nell'ultimo brano citato, il bambino biondo tradizionalmente raffigurato sulle scatole di Kinder Cioccolato.

Un altro esempio:

Sì, sì, una bella offertina, ci vuole. E poi si compra la 164 al Piergiangi, ché ormai c'ha ventiquattr'anni e non potrà mica andare tutta la vita in golf, no?, ché la golf la si passa alla Maria Stuarda Betty, ché ormai fa i diciotto anche lei!... (Brizzi 1994: 47)

In questo brano l'amara ironia del narratore smaschera l'ipocrisia dei partecipanti alla messa domenicale nominando modelli di automobili che sono simbolo di diversi status socioeconomici. ${ }^{10}$ Come in Moccia, pertanto, anche in Brizzi le marche contribuiscono a costituire un sistema di valori entro il quale si muovono i personaggi; ma la disamina rimane implicita, celata dietro l'allusione ai nomi di marca.

10 Per esempi simili cf. Sini (2014: 2317). 
Negli esempi di Brizzi sopra riportati, e in opposizione ai brani di Moccia, i nomi commerciali si contraddistinguono per due caratteristiche: dal profilo semantico, si tratta di nomi evocati per il loro valore allusivo, per la loro portata culturale, non primariamente per il referente materiale; dal profilo narrativo, sono nomi che, con Olsen \& Lanseng (2012), si possono definire highly plot-integrated, ossia portatori di una funzione all'interno della narrazione. In prospettiva sintattica si osserva inoltre la loro combinazione con elementi non prevedibili, come sarebbe "chitarre Fender" rispetto al più originale "mondo Fender" che ricorre in Brizzi (1994).

Lo stesso vale per il brano che segue:

Due settimane in Inghilterra, e tra la preoccupazione di trovare la corriera giusta per Heathrow e il viaggio di ritorno verso Londra, erano rimasti incastrati solo il corso d'inglese e le facce tipo Benetton di Paulos, Ivan, Shoko e di tutti gli altri amici conosciuti davanti ai toast di pollo nella canteen della scuola (Brizzi 1994: 128).

Ancora una volta sono necessarie determinate conoscenze culturali per capire il riferimento con cui sono qui fugacemente descritti gli amici del protagonista. Si allude, com'è noto, alle campagne pubblicitarie dell'azienda Benetton negli anni Ottanta, che esaltano il valore della diversità raffigurando giovani di diverse etnie amichevolmente mescolati, resi dal narratore attraverso nomi dal trasparente rimando geolinguistico. ${ }^{11}$ Il marchionimo Benetton è impiegato per le sue caratteristiche connotative più che per quelle denotative: si allude a un sistema di riferimenti culturali e conoscenze condivise, quindi, in ultima analisi, a luoghi comuni. Al contrario, in Moccia lo stesso nome di marca è utilizzato in maniera puramente referenziale: "Da Benetton l'aspetta una giacca con una gonna bordeaux" (Moccia 2004: 75). Inutilmente si cercherebbero, nell'opera del romano, casi di "mondo Fender" o di "bambini nazisti della Kinders".

11 Cf. l'es. riportato da Vaxelaire (2005: 219): “une «famille-Benetton»", glossata nel testo stesso con "des enfants de toutes les couleurs". 


\subsection{Sistema della moda, sistema della lingua. Tratti e valori}

I nomi commerciali citati nei brani di Brizzi manifestano un'altra peculiarità: non sono sostituibili. Clark's è Clark's perché il nome fa riferimento a un preciso fenomeno culturale localizzato temporalmente; lo stesso vale per Fender e Benetton. I marchionimi utilizzati referenzialmente, invece, sono perlopiù intercambiabili con altri; sono necessari alla narrazione, genericamente, in quanto marche, non in quanto si tratta di quelle marche specifiche.

Proprio questo avviene nel passaggio dalla prima versione di Tre metri sopra il cielo (1992), ambientata verso la fine degli anni Ottanta, alla seconda (del 2004), in cui la storia si svolge agli inizi del Duemila. In dettaglio sono stati sostituiti ventitré nomi commerciali diversi, ventuno dei quali ricorrono in costrutti argomentali: "un Maggiolone" diventa "una New Beetle", "un Boxer" diventa "un Free", dai "Ballorama" (sic) si passa ai "Ray-Ban". Di questi ventun costrutti, nove sono usi metonimici del nome d'impresa per il nome di un suo prodotto specifico: "una Camel", per es., è sostituita da "una Marlboro".

Si tratta di sostituzioni puntuali che non comportano cambiamenti di significato a livello globale; l'unica variazione è quella di un ipotetico "di moda" opposto a un "fuori moda" rispetto al tempo della storia e, ancor più, al tempo della pubblicazione. Il rimpiazzo può avvenire senza colpo ferire in quanto gli elementi sostituiti non sono funzionali alla vicenda narrata; non sono integrati nel testo in maniera sistemica, per cui si possono modificare senza che sia necessario operare altri cambiamenti.

Tali cambiamenti, insieme ad altri operati su aspetti tanto stilistici (passaggio dal passato al presente per i tempi verbali) quanto contenutistici (per es. l'aggiunta, nella trama, di telefoni cellulari), si inseriscono in un'operazione editoriale e commerciale di ammodernamento del romanzo. Ogni scrittore, infatti, si trova a fare i conti con lo scorrere del tempo; tra i nomi propri, soprattutto i nomi commerciali, 
che più rapidamente degli altri finiscono nell'oblio, ${ }^{12}$ fungono da spia di una precisa collocazione temporale, vanificando lo sforzo compiuto per essere attuali a tutti i costi.

Però non tutte le strutture con marchionimi si deteriorano alla stessa velocità, perdendo il riferimento immediato e cadendo dalla memoria collettiva di una società. Si è già accennato al maggior grado di opacità degli usi argomentali rispetto a quelli predicativi, che contengono un riferimento esplicito al nome generico del prodotto (l'osservazione, naturalmente, si limita agli usi referenziali, gli unici presenti nell'opera di Moccia). Si confronti lo stesso passo così come appare nelle due versioni del romanzo:

Una bella ragazza mora, dagli occhi verdi e un bel sedere rotondo, imprigionato in dei crudeli Avirex, sorrise all'amica, una biondina alta come lei ma un po' più tonda. (Moccia 1992: 20)

Una bella ragazza mora, dagli occhi verdi e un bel sedere imprigionato in $\mathrm{cru}$ deli Miss Sixty, sorride all'amica, una biondina alta come lei ma un po' più tonda. (Moccia 2004: 18)

Perché questo cambiamento? Si può ipotizzare, guardando al referente, che $i$ jeans Avirex non fossero più di moda nel 2004, quindi il personaggio doveva indossare dei jeans Miss Sixty per sembrare in; oppure, più sottilmente, si dirà che il riferimento ellittico ("degli Avirex" per 'jeans di marca Avirex'), basandosi su conoscenze condivise non più così diffuse - in particolare presso il pubblico target del romanzo -, non era sufficientemente trasparente. Come afferma Coletti (2010), "I concorrenti che perdono sul mercato escono anche dalla lingua"; meglio, smettono di essere un luogo comune condiviso. La marca Avirex, in effetti, ricorre ancora nell'edizione più recente del romanzo ma solo in funzione attributiva, nell'espressione "giubbotti Avirex" (Moccia 2004: 22). Nel passaggio da "crudeli Avirex" a "crudeli Miss Sixty", quindi, è in gioco un solo valore linguistico del marchionimo, un solo luogo comune passato di moda, quello per cui gli Avirex sono dei jeans.

12 Sini (2014: 2313) si domanda, et pour cause, se la comprensione dei romanzi contemporanei, soprattutto a distanza di tempo, non sarà ostacolata dal frequente ricorrere di "ces éléments indigestes, à l'étymologie obscure". 
Lo si è già osservato: sono proprio le strutture argomentali a registrare il maggior numero di sostituzioni nel processo di aggiornamento del romanzo. La ragione è chiara se si considera che, in un'ottica linguistica, la conseguenza dell'uso nominale di un marchionimo reso ancor più opaco dal passare del tempo è l'incomprensione, sia pure solo momentanea (il contesto fornisce di regola gli estremi per comprendere il riferimento). Un costrutto come i Balorama scade presto; è questo il motivo - essenzialmente linguistico - per cui, nella nuova versione del romanzo, esso è stato sostituito con $i$ Ray-Ban.

Alla luce di queste osservazioni acquista un altro valore quanto afferma Zardo (2007: 253) a proposito dei marchionimi, ovvero che "si tratta di parole dotate [...] di grande mobilità e alta mortalità": i nomi di marca sono effimeri non perché, semplicemente, scompaiono dalla lingua, ma perché perdono l'univocità del riferimento, soprattutto nei casi in cui nomi d'impresa sono usati come designazioni di un prodotto specifico. In questo modo, ovvero in absentia, si manifestano nella lingua i tratti "di moda" e "fuori moda": come (mancata) condivisione di un luogo comune.

\subsection{Le massaie di Migliorini e la nascita di luoghi comuni}

Migliorini è stato tra i primi a intuire l'esistenza di una relazione tra uso nominale dei marchionimi e luogo comune:

quando l'estratto Liebig era appena introdotto in commercio, non si poteva parlare che di estratto Liebig, e solo più tardi, quando esso fu più largamente noto, dapprima le massaie e poi altri avranno detto il liebig: ormai, dopo una convivenza abbastanza lunga da impregnare la secondo [sic] parola del significato della prima, questa prima diventava, almena [sic] in un certo ambiente, superflua, come espressione del genere accanto a quella della specie (Migliorini 1927: 92).

La caduta del nome comune sarebbe quindi una riduzione di ridondanza, avvenuta a seguito del trasferimento di significato dal nome comune al nome proprio (v. §1.1.4). La ridondanza, però, è tale in riferimento a un 
insieme di parlanti che condividono, scrive Migliorini, la stessa "lingua speciale", per i quali tale trasferimento di significato - oltre che la diffusione di un prodotto - avviene prima che per altri. Un luogo comune linguistico, come osservato nel $\$ 7.2$, può essere di moda o fuori moda; esso, in ogni caso, nasce come relativo (a un gruppo di parlanti) e non per forza diventa un luogo comune perfetto e generalizzato, ovvero un nome comune (cf. La Fauci 2011: 216-219), inscrivendosi nella memoria individuale e collettiva (cf. Sini 2014: 2315).

Prima dell'eventuale generalizzazione del luogo comune, l'uso argomentale del marchionimo - il liebig - è opaco e il riferimento non è univoco. C'è chi capisce e chi è fuori dal gruppo; l'espressione fa parte della lingua di una koinè, una comunità che condivide conoscenze extralinguistiche. ${ }^{13}$ Di conseguenza il discrimine non si pone più tra chi possiede un determinato feticcio e chi no, come per i personaggi di un romanzo; quando entrano in gioco i marchionimi - simulacri dell'oggetto reale, per riprendere Barthes ${ }^{14}$-, la differenza è tra chi capisce il riferimento e chi non lo capisce, tra chi conosce la marca e chi non l'ha mai sentita nominare (non tutte, come sanno gli esperti di marketing, hanno lo stesso grado di brand awareness). ${ }^{15}$

In letteratura, la questione coinvolge il lettore e diventa perciò un problema di target: qual è il pubblico a cui, idealmente, l'autore si riferisce? Qual è l'obiettivo perseguito attraverso l'uso (insistente) di marchionimi: allontanare o coinvolgere? Per comprenderlo è utile osservare la tipologia morfosintattica delle strutture in cui ricorre il nome di marca: usi argomentali, in particolare metonimici, come si è visto,

13 Sulla questione dei marchionimi come elementi che contribuiscono alla costruzione identitaria di una comunità cf. anche Sini (2014: 2313-2314).

14 Scrive Barthes (1967: 899-900) nel saggio Système de la Mode: "Pour obnubiler la conscience comptable de l'acheteur, il est nécessaire de tendre devant l'objet un voile d'images, de raisons, de sens, d'élaborer autour de lui une substance médiate, d'ordre apéritif, bref de créer un simulacre de l'objet réel, en substituant au temps lourd de l'usure, un temps souverain, libre de se détruire lui-même par un acte de potlatch annuel."

15 D'altro canto, come osservano Botton, Cegarra \& Ferrari (2002: 86-87), il valore della griffe si estende oltre la cerchia dei consumatori diretti: "L'oggetto firmato deve poter essere riconosciuto [...] anche da tutti quelli che non possono o non vogliono accedervi, poiché la firma costituisce un criterio di differenziazione sociale". 
richiedono una certa dose di conoscenze - determinate da fattori storici e socioculturali ${ }^{16}$ - perché sia possibile riconoscerne immediatamente il riferimento. Scrivendo "anfibi doctor marten's", Brizzi (1994: 32) dà la possibilità a tutti i lettori, o perlomeno a chi conosce questa particolare accezione di anfibi, di cogliere il rimando a un tipo di calzatura; quando Moccia (1992: 317) scrive "le Tobacco", invece, esclude dalla comprensione diretta chi non condivide il luogo comune per cui "le Tobacco" sono un paio di scarpe.

Come influisce su questo la dimensione temporale? L'espressione che lega un nome di prodotto e un nome di marca, se ha modo di diffondersi per un tempo sufficientemente lungo, può subire riduzione di ridondanza, nella prospettiva adottata da Migliorini; lo scorrere del tempo, indebolendo e rendendo meno necessario il legame con il nome generico implicito, restituisce opacità al costrutto e fa sì che questo, inevitabilmente, decada, così come il prodotto che esso denomina è passato di moda. Chi usasse tali costrutti una volta che non sono più in circolazione, oltre a non essere compreso, farebbe la figura del démodé. Forse è questa la ragione profonda della sostituzione di alcune marche da parte di Moccia. Invece di "una 155", meglio parlare di "una vecchia Punto"; l'aggettivo esprime quello che, lasciando il riferimento precedente alla marca, sarebbe stato implicito (nel 2004 l'Alfa Romeo 155 era un modello vecchio, mentre non lo era nel 1992), ma prescindendo dalla volontà del narratore.

\section{Conclusioni}

Le scelte linguistiche operate dall'autore di Tre metri sopra il cielo, in particolare la sostituzione di singoli nomi di marca dalla prima alla seconda versione del romanzo, lungi dall'essere un fenomeno puntuale d'importanza secondaria, appaiono come un riflesso di come egli

16 Infatti, a dispetto di quanto sostiene Mattioda (2008: 110), non sempre i significati veicolati dalle marche sono in grado di superare le barriere generazionali (oltreché culturali). Al proposito cf. Matt (2007: 248). 
concepisce la propria opera letteraria: un prodotto di consumo sottoposto alle mode, al loro mutare nel tempo, né più né meno di un capo d'abbigliamento o, appunto, di una marca che diventa démodé. Sono scelte giustificate solo in relazione al presente, alla contingenza della pubblicazione, al sistema di mercato editoriale che richiede una riattualizzazione dei suoi prodotti più commerciali. Studiando aspetti strutturali che coinvolgono le ricorrenze di marchionimi si deduce che a Moccia non interessa chiamare in causa un tempo lungo o persino, riprendendo Hemingway, fare i conti con l'eternità, ${ }^{17}$ come avverrebbe con riferimenti onomastici meno puntuali e, soprattutto, con la loro integrazione nel flusso della narrazione, alla stregua di quanto avviene in Brizzi.

Tre metri sopra il cielo si presenta, da questo punto di vista, come opera letteraria perennemente caduca, dal momento che il tempo della storia è aggiornato in funzione del tempo della pubblicazione. Quest'ansia di contemporaneità coinvolge importanti aspetti stilistici e narratologici, il più appariscente dei quali è il mutamento dei tempi verbali dal passato della prima versione al presente della seconda. Inoltre nella versione del febbraio 2004 compare un riferimento alla canzone Sere nere di Tiziano Ferro, datata novembre 2003 (cf. Moccia 2004: 34). Di conseguenza la vicenda, che si svolge in un arco di tempo che va grossomodo da marzo a dicembre, è ambientata nello stesso anno di pubblicazione. Mirabile visu, per i lettori del primo minuto il romanzo ha luogo addirittura nel futuro.

Caratteristica dei marchionimi nel romanzo d'esordio di Moccia, in una prospettiva linguistica, è il loro frequente ricorrere in costruzioni argomentali, senza nomi comuni riferiti al prodotto generico. In tal modo le marche si fanno traccia della formazione di luoghi comuni, di un'identità collettiva a volte posticcia e sempre provvisoria, che muta con il mutare delle stagioni della moda. Come le massaie di Migliorini, gli ipotetici lettori ideali di Tre metri sopra il cielo devono potersi ritrovare in tali strutture argomentali e nei loro riferimenti impliciti. La capacità di comprendere immediatamente tali riferimenti è un equivalente linguistico dei feticci che deve possedere chi vuole sentirsi parte della "tribù".

17 Ernest Hemingway, Banquet Speech, 1954 (<www.nobelprize.org/nobel_ prizes/literature/laureates/1954/hemingway-speech.html>, 3.6.2015). 
Nel loro conformismo perbenista, desideroso di adeguarsi ai dettami del gruppo che li vuole griffati dalla testa ai piedi, i personaggi di Moccia - per riprendere una definizione tratta da Jack Frusciante sembrano usciti da un rapporto sui giovani a cura della Comunità Europea e confermano i più corrivi pregiudizi sull'adolescenza, in particolare il valore supremo dell'apparire e del vestirsi di marca. Nel romanzo mocciano, d'altro canto, i nomi di marca hanno funzione puramente denominativa e referenziale, mancando invece del tutto la dimensione ironica e metanarrativa implicata da Brizzi attraverso l'allusione a un complesso sistema di valori; tutto è come appare, non c'è un altro piano di lettura.

Qual è, in conclusione, il significato profondo dell'ingombrante presenza dei marchionimi in Tre metri sopra il cielo? Come emerge dal confronto tra le due versioni del libro, il sistema è coerente e si manifesta, letteralmente, come narrativa di consumo: un prodotto che scade. Vittima non innocente della tirannia del tempo, il romanzo riflette lo stesso meccanismo che presiede al perenne variare della moda, sistema di conoscenze variabili nello spazio e nel tempo, condivise da un gruppo sociale e soggette a giudizi di valore. La sostituzione delle marche è spia dell'adesione a una politica dell'attuale a tutti i costi, una strategia editoriale di mercato per cui il libro è un prodotto che va aggiornato nei suoi elementi più caduchi, nella fattispecie i riferimenti culturali. Seguendo la diffusa moda della giovinezza - eterna, questa sì -, la prima edizione del romanzo è stata rivista per poter vendere un prodotto che piacesse alla nuova koinè di giovani e adolescenti che nel frattempo, tra computer e cellulari, era venuta alla ribalta. Il risultato, malgrado le intenzioni, è un lifting imperfetto, incapace di nascondere l'età di un prodotto che già alla nascita, non da ultimo nell'uso reiterato di marchionimi non essenziali alla narrazione, portava i segni manifesti della propria caducità. 


\section{Consuntivo dell'analisi}

"Basta la parola". Lo storico slogan dei confetti Falqui sembra implicare, in linea con gli studi di marketing ai quali si è accennato nella Parte I, che il solo nome sia sufficiente per convogliare un insieme di significati, valori, associazioni. Chi andrà in farmacia potrà chiedere, semplicemente, Falqui: non "confetti Falqui" o "pasticche lassative prodotte dalla ditta chiamata Falqui".

Gran parte della bibliografia sui nomi commerciali, passata in rassegna nella Parte I, li analizza "alla Falqui": considerando, cioè, solo la parola, il nome isolato. Al di fuori dell'idealizzazione pubblicitaria, però, i marchionimi ricorrono spesso all'interno di proposizioni, manifestando proprietà distribuzionali e combinatorie differenti, che sono state investigate nei capitoli che precedono. Non stupisce, allora, che l'analisi morfosintattica - uno studio di brand integration, lo si è denominato nell'Introduzione - porti a risultati in parte divergenti da quanto è stato elaborato sui nomi di marca in una prospettiva ontologica, in merito per es. alla questione categoriale o a quella del significato.

In queste pagine si riassumono gli aspetti principali emersi dall'analisi, prima di chiudere il lavoro, nelle Conclusioni, con una riflessione di carattere generale che interpreti i risultati alla luce delle ipotesi esposte in apertura. Tale breve panoramica mette in luce la varietà $\mathrm{e}$ complessità dei risultati che scaturiscono dalla descrizione dei nomi commerciali in effettivi contesti di ricorrenza, rispetto a trattazioni che argomentano sulla base di esempi (fittizi) limitati alle strutture più evidenti, in cui i nomi commerciali compaiono in funzione quasi esclusivamente argomentale. 


\subsection{Nessi nominali: usi argomentali}

A livello di nesso nominale si sono per prima cosa distinti i costrutti in cui il marchionimo ricorre in funzione argomentale da quelli in cui si presenta come modificatore, con funzione predicativa (v. §8.2); due tipologie di costrutti, lo si è visto più volte nel corso dell'analisi, che possono stare in rapporto di equivalenza.

In merito agli usi argomentali, l'analisi ha indagato i rapporti tra il nome commerciale come nucleo del nesso e la sua determinazione e modificazione.

La presenza di articoli è sintatticamente motivata quando coricorrono dei modificatori (attributi, possessivi ecc.): "Mirzeta [...] stringe tra le mani la sua Barbie, una Barbie anche lei scampata alla guerra". Nei restanti casi si è approfondito se i nessi determinati stiano in variazione libera con altri non determinati.

Il fenomeno della determinazione come alternanza libera emerge con particolare intensità in relazione ai marchionimi, quasi ne fosse un tratto caratterizzante. Ci si riferisce in particolare a ricorrenze con funzione di soggetto, per le quali l'alternanza si pone di frequente tra articolo la e Ø. Si nota una concomitanza con il carattere [+ umano] del marchionimo, osservabile in correlazione con predicati verbali che legittimano un soggetto umano. Tali ricorrenze sono usi metonimici del nome commerciale: il nome di marca per le persone che lavorano nell'azienda omonima.

Si sono quindi trattati i nomi commerciali sistematicamente determinati: le Adidas, i Ray-Ban, la Dedra. Piuttosto che parlare di articolo obbligatorio è opportuno riferirsi qui a una pertinenza della determinazione, che in alcuni casi si manifesta con differenze di valore legate alla variazione del genere o numero manifestati dall'articolo: la Superga/le Superga. In contesti non-marcati l'articolo si incontrerà regolarmente, in altri no: quando il marchionimo ha valore di plurale indeterminato ("Armani sfoggia spesso Nike bianche"); in elenchi ("vivono a pane, Coca-Cola e software"); con determinate preposizioni ("il presidente Eisenhower in Lacoste", "milioni e milioni di Sony"). In altri casi, per es. con i marchionimi Vespa o Nutella, ricorrono usi non-determinati 
anche in funzione di soggetto, che suonano piuttosto marcati; sono usi - in genere da parte della ditta produttrice - che mirano a rafforzare il valore propriale del nome commerciale.

Dall'analisi emerge una sostanziale novità: per l'italiano, presenza, rispettivamente assenza di determinatori non rappresentano un criterio che permetta di distinguere univocamente tra nomi di prodotto e nomi d'impresa. In maniera più affidabile si può invece stabilire se la determinazione costituisca variante libera e con che cosa alterni (paradigmaticamente): con articoli indeterminativi, con articoli plurali e così via.

Rispetto agli usi in funzione di soggetto, l'alternanza in posizione postverbale non è libera: vi è una differenza tra "Altobelli calza Adidas" e "Helmut Newton calza Reebok che sembrano nuove", in cui il nome commerciale ha valore nominale (plurale indeterminato), mentre Adidas, nel primo esempio, ha valore avverbiale. Il contesto sintattico permette di volta in volta di specificare il valore del marchionimo: nel caso di "tracannare Guinness", citato in un elenco di attività, si propenderà per l'interpretazione argomentale (= oggetto diretto); nel caso di "mia zia [...] compera solo Barilla" o "Benigni [...] veste Armani" è probabile si tratti di un uso avverbiale del marchionimo (per il quale v. infra, §8.4).

Alcune alternanze di maschile e femminile si sono rivelate funzionali alla distinzione di valori diversi del nome commerciale: una Yamaha/uno Yamaha, il Peugeot/la Peugeot. Altre alternanze sono libere: un/una Ford Transit, i/le Birkenstock. Il femminile (plurale) ricorre con regolarità in correlazione al valore di titoli di un'azienda ("le Guinness sono calate di 7,5 punti"), mentre il femminile singolare si correla al valore di 'azienda'.

Il numero si presta meno del genere a differenziare valori; si consideri l'alternanza tra la/le Marlboro, addirittura tra la/le Superga in alcuni contesti; i Ray-Ban ricorre, nei dati, solo al plurale. Anche per la categoria del numero si riscontrano casi di variazione libera: "(giocare con) il/i Lego, la/le Barbie".

Un accenno agli articoli indeterminativi. Nei costrutti in cui vi è alternanza libera tra articolo determinativo e $\varnothing$ (valore di 'impresa'), il loro ricorrere dà luogo a usi con valore antonomastico: "Quando si 
dice occhiali, non c'è invece una Nike, o una Levi Strauss" (in questo simili a usi con articolo determinativo plurale: "Non si fa sport con le Coche Cola, le Nestlé, le McDonald's"; o con articolo determinativo singolare e modificazione: "la Fiat della scarpa da sci"). Combinati con modificatori a valore restrittivo, gli articoli indeterminativi danno invece origine a usi cosiddetti modalizzanti del nome proprio (GaryPrieur 1991): "una Fiat non italiana non sarebbe concepibile". In altri casi, articoli determinativi e indeterminativi sono intercambiabili senza che ciò cambi il valore del nome commerciale: "la/una Lacoste rossa", "la/una Vespa".

Quanto alla modificazione, si sono riportati vari casi di marchionimi modificati da aggettivi e altri attributi: "la Uno bianca", "un Toblerone da un chilo", "una Lancia Dedra". Si è riscontrato che attributi preposti possono manifestare valore non-restrittivo ("i mitici Levis", "la danese Lego"), di contro al valore restrittivo che presentano attributi posposti: "la Olivetti danese", "la Migros di Sant'Antonino".

Ricorrono casi di apposizioni interne ("il colosso americano Kodak"), tra le quali è possibile annoverare anche strutture come "l'azienda Prada" e "le sigarette Marlboro" in virtù della relazione con $\mathrm{i}$ costrutti ad apposizione esterna "La Lines, industria tutta italiana" o "la Marlboro (la sigaretta più venduta)". I marchionimi vi ricorrono come argomenti dei quali si predica qualcosa: in primo luogo l'essere un'industria, una sigaretta.

\subsection{Nessi nominali: usi predicativi}

Gli usi predicativi $(N N m c)$ sono suddivisibili in due gruppi a seconda del tipo di relazione che si instaura tra marchionimo e nome modificato: si tratta di una predicazione denominativa o no? Uno stesso costrutto, per es. l'azienda Fiat, si presta a ricorrere in entrambe le versioni: con Fiat come predicato di denominazione, e vi sarà allora un'equivalenza con il nesso "(la) Fiat" (= 'Fiat è un'azienda'); con Fiat come aggettivo di relazione ("fiatese"; cf. "la ricetta benettoniana"), e la corrispondenza 
sarà allora con il nesso con complemento preposizionale "l'azienda di/ della Fiat" (= 'del gruppo Fiat'). Seguendo il suggerimento di La Fauci (2009: 158), si propone di trattare la prima struttura come un caso di modificazione di tipo denominativo, non come un'apposizione interna.

Anche nei costrutti "Det marca Nmc" il marchionimo funge da modificatore denominativo di marca; infatti non vi è equivalenza con "marca di Nmc" ma con costrutti ad apposizione esterna come "La Brahma è una marca di birra". Rispetto al nesso azienda Nmc si osserva qui un minor grado di equivalenza con l'uso argomentale Det Nmc nei casi in cui il nome (classificatore) marca non sia ripreso nel costrutto: forse perché tale valore non costituisce un luogo comune alla pari di 'azienda'; inoltre perché ipotizzare il valore di 'marca' nel caso di predicati verbali che legittimano un soggetto ristretto - decidere, volere ecc. - porterebbe a stabilire un processo metonimico più complesso che nel caso di 'azienda'.

Si hanno modificazioni denominative anche con nomi relativi a un prodotto, che ricevono un nome specifico in un atto di battesimo: "il sapone Camay", "lo yogurt Alleluya".

Marchio ha spesso valore di 'logo', 'segno grafico', casi in cui non funge da classificatore al pari di marca; perciò è pluralizzabile o ricorre con articolo indeterminativo.

In costrutti quali "la pasta Barilla", "un registratore Philips" o "una tuta Adidas", tra nome comune e marchionimo si riconosce un rapporto del tipo "prodotto-produttore". Può esservi coincidenza tra questi nessi e l'uso argomentale del marchionimo ("i Pampers"). Ciò non si riscontra, invece, per i nessi in cui ricorrono nomi comuni astratti: "caso Perrier", "obiettivo Fiat". Tra i due componenti del costrutto vige, a seconda dei casi, un rapporto di possesso e/o soggettivo ("Fiat ha un obiettivo'). In questi costrutti il marchionimo non modifica il nome comune in termini denominativi. Lo stesso vale per i nessi con nomi umani: "pilota Mercedes", "dirigenti Microsoft", "utenti Apple". Per tutti questi esempi, in cui il nome commerciale funge da aggettivo di relazione, vale la corrispondenza con il complemento preposizionale di(Det) Nmc: "una tuta della Adidas", "la risposta della Fiat", "l'operaio della Nissan"; con l'eccezione di usi traslati o metaforici: "mamme Pampers", "lavoratori kleenex", "femminismo Perugina". Nel caso di 
nomi comuni relativi a un prodotto, come si accennava, il modificatore marchionimico può nominalizzarsi: "l'Alleluya", "le Adidas", "una Lacoste", diventando argomento, da predicato che era; o meglio, modificando un argomento - l'articolo - che non costituisce, come si è visto sopra, variante libera. Se tale nominalizzazione non avviene, è per ragioni di conoscenze condivise, non di sintassi.

Nei nessi Det Nmc di questo tipo, dunque, l'articolo non costituisce il rimasuglio di un nome cancellato. Il concetto di ellissi è problematico, dal momento che presuppone un costrutto "integro", che sarebbe da ricostruire, dietro la struttura "ridotta"; inoltre non spiega in maniera economica i casi di alternanza libera di genere (i/le Birkenstock). Più plausibile è l'ipotesi di un processo di nominalizzazione alla base dei costrutti Det Nmc, che descriva i nomi di marca come predicati che si sono argomentalizzati. Ogni predicato è facilmente nominalizzabile quando l'argomento di cui è un predicato è noto o facilmente deducibile dal contesto: "È cosparso di Armani uomo". Si tratta di una trasformazione funzionale che non si riflette nel marchionimo a livello morfologico.

$\mathrm{Ne}$ emerge che i nomi commerciali, nei loro usi argomentali, sono processi, non enti. L'articolo costituisce l'argomento di cui il marchionimo è il predicato e, come un argomento, ne manifesta genere e numero. Lo stesso vale per i costrutti "gli alunni promossi e i bocciati" o "lo stesso scarto qualitativo che separa le tagliatelle della nonna da quelle Barilla", in cui il determinatore ha funzione pronominale. La differenza è che nel caso di le Nike, la Ford ecc. la relazione con un luogo comune è generalizzata e non si limita a un contesto proposizionale specifico.

\subsection{Nessi preposizionali}

I nessi in cui il marchionimo è introdotto da una preposizione sono stati distinti in nessi argomentali e modificazioni predicative (complementi del nome e del verbo). 
Hanno funzione argomentale nucleare i nessi preposizionali che compaiono nei costrutti "può solo giovare alla Fiat", "Young è stato assunto dalla Nike", ma anche i complementi preposizionali del predicato che ricorrono in "vivere di Kodak", "investire in Nike", "entrare alla Honda", questi ultimi due caratteristici dei marchionimi. La determinazione è variante libera nel caso dei nessi con funzione di oggetto indiretto e soggetto di costrutti passivi (e con investire in), non però nel caso di entrare in con valore di 'essere assunto da una ditta'. Entrare in/ nella compare nei dati con il valore specifico di 'comprare azioni', 'fare investimenti (in un'azienda)'.

Tra $\mathrm{i}$ nessi con modificazione predicativa spiccano, per frequenza e varietà di ricorrenze, i complementi di specificazione. Vi si riconoscono relazioni diverse: genitivi soggettivi o oggettivi - i costrutti "l'acquisto della Pavesi (da parte della Barilla)", "la decisione della Fiat" -; rapporto prodotto-produttore: "i cosmetici della Revlon" e "il tablet della Apple" (con alternanza di/della se il nome commerciale non ha carattere antroponimico: si veda invece "il vestito di Valentino"); rapporti di lavoro (professione, ruolo all'interno di una ditta) con nomi umani, per es. "il presidente della Coop", "portavoce di Henniez"; rapporto contenente-contenuto: "tre pacchetti di Marlboro"; funzione di aggettivo relazionale (con valore di possesso) nei casi di "l'obiettivo della Fiat", "la politica di Alitalia". In tutti questi casi, con l'eccezione delle relazioni tra contenente e contenuto e dei genitivi soggettivi/oggettivi, vi è in genere equivalenza con $N \mathrm{Nmc}$.

Un altro caso di modificazione predicativa è quello con preposizione da: "il pollice da Blackberry", "scrittore da baci Perugina"; il nome commerciale vi ricorre non determinato.

I supplementi predicativi presentano valore locativo ("in vendita alla Migros": con alternanza tra $a(D e t), d a$ e, più raramente, presso; "lavorare in/alla $N m c$ ") o strumentale/modale, con mezzi di trasporto o capi d'abbigliamento: "Giovanni Agnelli in Timberland", "in Lacoste"; "gite in lambretta", "in Harley-Davidson". In questi ultimi casi la preposizione non è articolata. 


\subsection{Sintassi della proposizione}

A livello di proposizione influisce sul valore del marchionimo la combinazione con il predicato verbale. Se si tratta di un verbum dicendi o di un altro predicato che legittima un soggetto ristretto, il marchionimo in funzione di soggetto presenterà il tratto [+ umano]: "Cosa dice Opel al riguardo?"; "la Fiat ha deciso di vuotare il sacco"; "L'incidente [...] pare causato dalla Bmw dei tedeschi". Con il predicato verbale comprare, a determinare il valore del marchionimo in funzione di oggetto diretto è anche il fatto che compaia o no un altro nome commerciale come soggetto: "Nestlé ha comprato Buitoni" (equivalenza con "la Buitoni") vs. "I veneti [...] hanno speso ben 25 miliardi per acquistare Jacuzzi" (con valore di plurale indeterminato).

I nomi commerciali ricorrenti in combinazione con essere svolgono funzione argomentale nel caso dei costrutti identificativi ("La prima a introdurre una svolta fu proprio la Ford"), mentre hanno funzione predicativa - similmente agli aggettivi - quando ricorrono come predicati nominali: "le lavatrici migliori del mondo sono Aeg o Miele"; "la Ferrari è Fiat", dove identificano in genere la proprietà 'essere di marca $N m c$ ' o 'appartenere alla marca/azienda $N m c$ '.

Gli usi avverbiali, per es. "Elton John veste Versace", non presentano alternanza libera con il marchionimo determinato; a volte, anzi, con predicati verbali che entrano prevalentemente in costrutti intransitivi ("volare Alitalia") il nome commerciale ha valore univocamente non-argomentale, quindi predicativo. La struttura è utilizzata per alludere ad azioni ripetute, a consuetudini nell'ambito dei consumi ("Gianni [...] fuma solo Marlboro") o a un'attività generica: "Naturalmente si mangia McDonald".

I nessi "targato/firmato Nmc" fungono da predicazione rispetto a un nome o nesso nominale: predicazione (latamente) denominativa, in particolare quando il marchionimo è, appunto, una firma ("il noto pullover firmato Missoni"). Anche con nomi commerciali che non sono firme o targhe in senso stretto l'uso dei participi targato e firmato mette in rilievo un processo attinente all'apposizione di un nome: "il gelato firmato Algida", "i computer targati Sony". Nei casi di "le bustarelle targate Fiat" o "lo tsunami digitale targato Kindle e iPad" 
vi è un'interpretazione traslata; il marchionimo ha valore soggettivo rispetto al nome modificato, non diversamente dagli usi come aggettivi di relazione. Vi è in questo una somiglianza con gli usi del nesso "(di) marca Nmc", che compare come modificatore ("un orologio marca Breil", "sigarette marca Marlboro"), anche di nomi non di prodotto: "una tangente di marca Fiat", "un messaggio di marca Montedison". Tanto marca quanto i participi targato/firmato sono considerabili alla stregua di classificatori - e, come tali, sono eventualmente superflui alla compiutezza del costrutto -; il nucleo del nesso è il nome commerciale, che ha valore di modificatore.

Ha funzione argomentale, invece, il marchionimo che compare, introdotto da alla, in combinazione con un predicato verbale alla terza persona plurale, con soggetto non manifesto (umano non specificato): "Il rodaggio è andato bene, dicono alla Fiat"; "non contenti alla Guzzi andarono avanti"; "alla Pirelli storcono il naso". Una struttura di questo tipo ricorre con nomi propri che hanno carattere [+ umano] solo per metonimia (oltre ai marchionimi, per es., anche "assicurano al Milan"), non con antroponimi: non ricorrerà "dicono al Mario" per "Mario dice". Il parallelismo con la funzione argomentale di soggetto è visibile nel seguente costrutto: "Sorride Nanni Tua, presidente di una piccola fabbrica di sci (110 mila paia all'anno) ad Occhieppo Superiore vicino Vercelli. Ma sorridono anche alla Diadora, 190 miliardi di fatturato". 



\section{Conclusioni}

Seguendo il percorso d'analisi proposto in queste pagine, il marziano curioso della lingua - e con lui, ce lo auguriamo, chi avrà avuto la pazienza di leggere sin qui - ha potuto osservare che in italiano le strutture in cui ricorrono nomi commerciali sono numerose e multiformi; ora riesce a intuire un po' meglio in che modo essi si combinano agli altri elementi della frase. Infine, ripensando a nessi come "dominio Aprilia", "rialzo targato Fiat" o "lavoratori kleenex", si rende conto che il significato di questi nomi è più complesso rispetto a quanto aveva potuto leggere nei dizionari o nelle enciclopedie.

Come scrive Kolde (1995: 400), non è sufficiente constatare che i nomi propri - in questo caso i nomi di marca - si differenziano largamente dai nomi comuni, rispetto ai quali sarebbero "deficitari" quanto a flessione e possibilità di ricorrere con modificatori. È necessario, continua lo studioso, approfondire la realizzazione concreta, lingua per lingua, del loro statuto grammaticale particolare. È quanto si è cercato di fare in queste pagine, relativamente ai nomi commerciali e al caso dell'italiano. Ne emerge l'impressione che la generale mancanza di flessione morfologica non impedisca ai marchionimi di ricorrere nei testi in un ventaglio di usi funzionali diversi; da questo punto di vista essi si dimostrano addirittura, per certi rispetti, più versatili degli stessi nomi comuni, richiedendo peraltro al linguista una maggiore perspicacia analitica e l'adesione coerente a una metodologia appropriata. Nella loro invarianza formale, infatti, i nomi commerciali mostrano all'ennesima potenza ciò che è valido per la globalità delle parole di una lingua: le loro proprietà - anche categoriali - non sono determinabili a priori; "divengono chiare invece (e nemmeno sempre) solo al momento in cui un insieme di relazioni combinatorie le precisa" (La Fauci 2011: 34). Per questo si è scelto un accostamento sintattico.

La ricerca si è posta come obiettivo primario la descrizione del funzionamento morfosintattico dei nomi commerciali in italiano, raggiungibile studiando $\mathrm{i}$ modi in cui essi ricorrono nel discorso e 
approfondendone le caratteristiche combinatorie. Per un riassunto delle strutture e delle proprietà morfosintattiche si rimanda al Consuntivo dell'analisi (cap. 8). I risultati ottenuti, lo si sottolinea un'ultima volta, sono validi solo per l'insieme di dati considerato e non hanno validità predittiva, bensì unicamente descrittiva. Nondimeno, le regolarità che sono venute alla luce si ritroveranno, almeno in parte, anche in altre tipologie testuali e nella lingua parlata (gli esempi tratti dal corpus LIP non sembrano divergere particolarmente da quanto osservato in relazione ai testi scritti). È un'ipotesi che meriterebbe di essere verificata.

Il secondo obiettivo cui si fa cenno nell'Introduzione ruota intorno al significato. Mentre nella bibliografia questo è correlato a un referente esterno, in base al quale si stabiliscono diverse categorie in cui suddividere i nomi commerciali, qui si afferma che il significato $\mathrm{o}$, saussurianamente, il valore - del marchionimo è dato dall'insieme delle proprietà combinatorie che emergono dall'analisi morfosintattica della proposizione. Tale valore si presenta come un riflesso superficiale delle relazioni presenti nel costrutto: una rete di relazioni sintagmatiche e paradigmatiche, che determina i vari nessi nella loro specificità e in opposizione ad altri. Non sono dunque i singoli nomi, in quanto appartengono a varie categorie referenziali (di prodotto, d'azienda ecc.), ad avere diverse proprietà combinatorie e semantiche; è la sintassi a farne scaturire valori diversi. In particolare la distinzione dei marchionimi nelle due categorie "nome di prodotto" e "nome d'impresa"/“di marca", riproposta di frequente nella letteratura, si rivela inadatta a rendere conto degli usi effettivi dei nomi commerciali nel discorso.

È ovvio: in alcuni casi l'analisi conferma una differenza, anche combinatoria, tra marchionimi che hanno valore di 'azienda' o di 'prodotto'. A chi ne deduca l'inutilità dello studio proposto si risponderà con l'affermazione perentoria di Harris (1952: 26):

Many of these conclusions may well have been obtainable intuitively without such formal analysis; but intuition does not yield results that are either explicit or rigorous.

Proprio questo si è cercato di offrire: una descrizione formale rigorosa ed esplicita della natura combinatoria dei nomi commerciali. 
L'analisi ha messo in luce i processi che intervengono dinamicamente nell'uso effettivo dei marchionimi. Un posto di rilievo spetta al processo definito di nominalizzazione, approfondito a più riprese nel corso dell'analisi, specialmente in merito alla relazione tra i costrutti $\operatorname{Det} N m c$ e Det $N N m c$ (usi argomentali e predicativi). Un esempio è, nel brano seguente, il nesso La Timberland:

\begin{abstract}
Un mondo a parte è quello delle agende in pelle "griffate". Hanno prezzi alti e qualità: la più cara è quella col marchio d'oltremanica Burberry's che nell'unica versione in pelle martellata sfiora le trecentomila. La Timberland, ora che ha gli importatori diretti, costa un po' meno rispetto all'anno passato (240 mila lire). (LaR)
\end{abstract}

In non pochi casi, gli usi nominalizzati raggiungono un grado tale di fissità da poter ricorrere anche laddove non vi sia coincidenza manifesta con un nesso nominale quale "agende in pelle" nell'esempio citato: le Nike, una Ferrari, i Levi's, la Nivea e numerosi altri costrutti hanno un valore relativamente stabile e univocamente determinato.

Il processo di nominalizzazione istituisce una relazione tra un luogo comune e la sua designazione (cf. La Fauci 2011: 217). In questo senso, le Nike e gli altri nessi appena evocati non sono, ontologicamente, dei nomi, bensì dei processi. Si può affermare lo stesso a proposito dei costrutti la Barilla (per l'azienda), la Vespa, l'Alleluya, ai quali soggiace un atto di battesimo, che per gli usi metonimici sopra riportati è solamente indiretto: si tratta di predicazioni, stavolta di tipo denominativo, che si argomentalizzano. La Barilla è 'l'azienda chiamata Barilla', la Vespa 'il ciclomotore battezzato Vespa' e così via. La determinazione che è presente - eventualmente come variante libera - in questi contesti non è un rimando implicito a un nome comune che sarebbe stato cancellato per riduzione di ridondanza (è l'ipotesi dell'ellissi); rappresenta invece l'argomento rispetto al quale il marchionimo funge da predicazione e che ne manifesta genere e numero.

Con questo si è giunti all'ultimo interrogativo posto nell'Introduzione, riguardante la natura linguistica dei nomi commerciali. Nella letteratura sul tema, lo si è ricordato nella Parte I, l'attribuzione categoriale dei marchionimi oscilla tra nome proprio e nome comune, giungendo 
non di rado ad assegnar loro una collocazione intermedia che, lungi dall'essere una soluzione elegante, in realtà non fa che aggirare il problema. In considerazione di quanto esposto sin qui si spera non suonerà come una boutade la proposta, emersa nel corso dell'analisi, di considerare invece i nomi commerciali come aggettivi: funzionalmente, s'intende, non da un punto di vista categoriale. Il marchionimo, infatti, partecipa della natura attributiva propria degli aggettivi, fungendo spesso da predicato (eventualmente denominativo) di un argomento (cf. pasta Barilla, marca Gillette, targato Fiat, vestire Prada); ma anche negli usi argomentali, ossia laddove può essere preceduto da un determinatore, è possibile riconoscere (lo si è constatato poco sopra) una funzione predicativa. Non si tratta dunque semplicemente di estendere a casi simili quanto osserva Platen (1997: 133) a proposito dello slogan di Auchan che contrappone "La vie austère" a "La vie auchan" [sic], in cui, scrive lo studioso, il nome di marca assume il ruolo di aggettivo qualificativo come sinonimo di 'abbondante' e 'opulento'. Ciò che in questo lavoro si denomina "aggettivo" è invece un valore processualmente determinato, un'ipotesi di fondamento funzionale, che va al di là del "ruolo" momentaneamente assunto dal marchionimo entro la proposizione e si basa su un'analisi non limitata a uno o a pochi esempi. Insomma, mentre i nomi comuni hanno una natura funzionalmente argomentale, tanto $i$ nomi propri quanto i nomi commerciali - che da questo punto di vista sono appunto definibili come nomi propri tout court, niente affatto "ai margini dell'onimia" - sono, funzionalmente, delle predicazioni.

Tale ipotesi e le osservazioni recate a sostegno della stessa, fondate su un ampio insieme di dati, si ritiene siano l'apporto più significativo della presente ricerca allo studio linguistico dei nomi commerciali. L'analisi ha inoltre contribuito a sfatare alcuni miti che ricorrono nella letteratura sui marchionimi, elaborati spesso in maniera astratta e senza disporre di una base di dati ricca e variata. Dal punto di vista metodologico, infine, si conferma l'utilità di un'analisi grammaticale, sintattica, per sondare le caratteristiche combinatorie dei nomi e le loro proprietà linguistiche; per cogliere, insomma, il fenomeno linguistico del nome di marca nel suo farsi. 
Chiaramente vi sono questioni che rimangono aperte: quale definizione dare dei nomi commerciali, tenuto conto della loro natura predicativa? È possibile dare una definizione prettamente linguistica o, come del resto in altri settori dell'onomastica di ben più antica tradizione, occorrerà riferirsi a categorie referenziali, per es. al ruolo che tali nomi svolgono in un contesto economico e commerciale? Ancora, è possibile stabilire una classificazione interna al settore dei nomi commerciali su base combinatoria?

Chi scrive è consapevole del fatto che, per ragioni di ordine pratico, la ricerca si è svolta su una casistica piuttosto ampia ma pur sempre minima rispetto alla totalità dei nomi commerciali. Nella Parte II si è precisato che l'analisi si concentra su marchionimi che, intuitivamente, si potrebbero definire prototipici, senza discorrere di categorie limitrofe, che andrebbero studiate separatamente e correlativamente: nomi di banche e altri servizi; nomi presenti sugli imballaggi dei prodotti, che tendenzialmente non ricorrono nei contesti testuali qui considerati; marchionimi utilizzati come nomi di squadre sportive, per citarne solo alcuni tipi.

Quanto ai corpora e ai dati raccolti, è ovvio che l'analisi potrebbe essere arricchita sia qualitativamente (diverse tipologie testuali), sia quantitativamente. Si ipotizza peraltro che le strutture e le proprietà distribuzionali dei marchionimi non ne uscirebbero grandemente modificate rispetto a quanto qui proposto, con l'eccezione forse degli usi pubblicitari.

Un discorso a parte merita la prospettiva diacronica, della quale in questa sede non si è tenuto conto, se non per osservazioni marginali. Un confronto tra dati di epoche diverse, attento anche all'aspetto statistico, consentirebbe di descrivere evoluzioni negli usi morfosintattici dei nomi commerciali e di osservare tendenze dell'italiano contemporaneo. Particolarmente promettenti, da questo punto di vista, sono la questione del determinatore come variante libera (oggi si tende a tralasciarlo) e la diffusione di alcune strutture: mentre il tipo " $N$ marca $N m c$ " pare ormai in disuso, continua a ricorrere l'uso avverbiale; la preposizione in prevale, in certi casi, su $a(D e t)$. 
Resta inoltre da approfondire il tema della variazione spaziale. I dati analizzati comprendono, lo si ricorda, articoli della rivista Spendere Meglio, edita nella Svizzera italiana; al proposito si è notato il ricorrere di presso laddove testi di provenienza italiana recano $a($ Det $)$ o da. Le osservazioni d'impronta diatopica potrebbero allargarsi alla questione del genere dei marchionimi (per es. con i nomi dei grandi distributori: il/la Denner) e al problema degli influssi di altre lingue sulle strutture e le ricorrenze di nomi commerciali, particolarmente interessante per la situazione elvetica.

Queste osservazioni conducono a un'altra fondamentale questione aperta: quanto descritto in questo lavoro vale per l'italiano ma sarebbe interessante, anche in un'ottica contrastiva, approfondire il funzionamento dei nomi commerciali in altre lingue. Se non mancheranno punti di contatto (usi avverbiali, per es., sono riconoscibili anche in tedesco), pure vi sono differenze; si pensi solo agli usi predicativi, che in parte trovano un equivalente, nelle lingue germaniche, nei nomi composti di due elementi, entrambi a valore nominale. Per domandarsi come funzionino linguisticamente i nomi commerciali in una data lingua non è necessario essere marziani; basta essere alloglotti.

In questa prospettiva, il progetto sui marchionimi può avere rilevanza anche al di fuori della comunità scientifica, siccome fornisce una descrizione di processi linguistici riguardanti nomi che non nascono nella lingua seguendo un'evoluzione tradizionale ma vi sono immessi di peso, per dir così dall'alto, con operazioni di marketing e attraverso i mass-media. Dei marchionimi, però, il parlante si appropria, integrandoli nella sintassi della lingua. I nomi commerciali sono resi produttivi a prescindere dalle intenzioni delle imprese, a volte persino esplicitamente contro di esse (i casi di genericizzazione, i vari to xerox e googeln). La ricerca permette ai parlanti non tanto di soddisfare curiosità sulle origini e le motivazioni onomastiche dei marchionimi, come fanno altri lavori di stampo più lessicografico, quanto piuttosto di comprendere meccanismi della lingua di cui essi fanno uso ogni giorno, benché in maniera spesso inconsapevole.

Quanto emerso attraverso l'analisi sintattica potrebbe risultare utile anche per le discipline che studiano la marca in prospettiva economico-commerciale. Se è vero che la nuova frontiera del marketing 
è il brand placement, l'inserimento (a pagamento) di marchionimi in opere di narrativa, cinematografiche ecc., non sarà superfluo sapere com'è percepito dal pubblico l'uso delle diverse strutture linguistiche contenenti marchionimi. Gli esempi di Moccia e Brizzi hanno mostrato infatti che gli usi metaforici e allusivi sono radicati nella narrazione e generalmente indispensabili per lo svolgimento della vicenda, mentre gli usi nominali e referenziali sono più facilmente valutati come superflui per la narrazione, ingenerando un sentimento negativo nei lettori. 



\section{Bibliografia}

Adjouri, Nicholas $2014^{2}$. Alles was Sie über Marken wissen müssen. Leitfaden für das erfolgreiche Management von Marken. Wiesbaden: Springer Gabler ( $1^{\text {a }}$ ed. 2004).

Ainiala, Terhi / Saarelma, Minna / Sjöblom, Paula 2012. Names in focus. An introduction to Finnish onomastics. Helsinki: Finnish Literature Society.

Anreiter, Peter 2002. Pharmakonyme. Benennungsmotive und Strukturtypologie von Arzneimittelnamen. Wien: Edition Praesens.

Arcamone, Maria Giovanna (a c. di) 2008. I nomi nel tempo e nello spazio. Atti del XXII Congresso internazionale di scienze onomastiche, Pisa, 28 agosto - 4 settembre 2005. Pisa: ETS.

Baldini, Massimo (a c. di) 1987. Le fantaparole. Il linguaggio della pubblicità. Roma: Armando.

Ballon, Gabriël-Luc 2007. La protection des noms choisis pour exercer une activité économique: l'approche juridique. In Kremer/Ronneberger-Sibold (2007a), 61-86.

Bartezzaghi, Stefano 2010. Non se ne può più. Il libro dei tormentoni. Milano: Mondadori.

Barthes, Roland 1967. Système de la Mode. In Id. (2002), Oeuvres complètes, vol. II: 1962-1967. Paris: Seuil, 895-1231.

Benveniste, Emile 1956. La nature des pronoms. In Id. (1966), Problèmes de linguistique générale, vol. I. Paris: Gallimard, 251257.

Berger, Dieter 1976. Zur Abgrenzung der Eigennamen von den Appellativen. Beiträge zur Namenforschung - Neue Folge. 11, 375-387.

Bergien, Angelika / Kremer, Ludger / Zilg, Antje 2008. Commercial names as indicators of innovation and change: new reflections and challenges. Onoma. 43, 7-23.

Boerrigter, Reina / Nijboer, Harm (a c. di) 2012. Names as Language and Capital. Proceedings "Names in the Economy" III, Amsterdam, 11-13 June 2009. Amsterdam: Meertens Instituut. 
Botton, Marcel / Cegarra, Jean-Jack / Ferrari, Béatrice 2002³. Il nome della marca. Creazione e strategia di naming. Milano: Guerini (1ª ed. 1996).

Brendler, Andrea / Brendler, Silvio (a c. di) 2004. Namenarten und ihre Erforschung. Ein Lehrbuch für das Studium der Onomastik. Hamburg: Baar.

Brizzi, Enrico 1994. Jack Frusciante è uscito dal gruppo. Ancona: Transeuropa. (Si cita dall'ed. Milano: Baldini\&Castoldi, 1995).

Calabrese, Omar 1974. Il marinismo in serie. Una lingua tra neo-arcaismo e paleo-neologismo. In Pignotti Lamberto, Il supernulla. Ideologia e linguaggio della pubblicità. Firenze: Guaraldi. (Ripubbl. in Chiantera 1989, 159-178).

Caprini, Rita 1976. Etimologia e storia di marca, marco, marchio, marcare, marchiare. Lingua nostra. 37, 74-76.

Chiantera, Angela 1989. Una lingua in vendita. L'italiano della pubblicità, presentazione di M.L. Altieri Biagi. Roma: La Nuova Italia scientifica.

Cignetti, Luca 2010. Apposizione. In Enciclopedia dell'Italiano. Online: <www.treccani.it/enciclopedia/apposizione_(Enciclope dia_dell'Italiano)/> (7.11.2014).

Coletti, Vittorio 2010. Marchionimi e nomi commerciali in terminologia. In Atti Convegno Assiterm 2009. Publifarum, 12. Online: $<$ www.publifarum.farum.it/ezine_articles.php?art_id=162> (20.11.2011).

CoLFIS = Corpus e Lessico di Frequenza dell'Italiano Scritto. Online: $<$ esploracolfis.sns.it/EsploraCoLFIS/> (24.9.2014).

Collins, Leslie 1977. A Name to Conjure With. European Journal of Marketing. 11/5, 340-363.

CORIS = CORpus di Italiano Scritto. Online: <corpora.ficlit.unibo.it/ TCORIS/> (24.9.2014).

Cotticelli Kurras, Paola 2007. Die Entwicklung der hybriden Wortschöpfungen bei den italienischen Markennamen. In Kremer/ Ronneberger-Sibold (2007a), 167-185.

Cotticelli Kurras, Paola 2008. La struttura morfologica dei marchionimi italiani nel XX secolo (fino agli anni '80). In Arcamone (2008), 695-709. 
Cotticelli Kurras, Paola 2011. «Spieglein, Spieglein an der Wand, wer ist die mächtigste, zuverlässigste, traditionsreichste Produktklasse im ganzen Land?» - Assoziationen bei italienischen Markennamen. In Kazzazi, Kerstin (a c. di), Eichstätter Sprachgeschichten. Ein Kolloquium zu Ehren von Elke Ronneberger-Sibold, Eichstätt 2010. Würzburg: Königshausen und Neumann, 95-113.

Cotticelli Kurras, Paola 2014. Italian Brand Names through their Associations: Current Trends. In Tort i Donada/Montagut i Montagut (2014), 449-458.

Cotticelli Kurras, Paola / Ronneberger-Sibold, Elke / Wahl, Sabine (a c. di) 2012. Il linguaggio della pubblicità italiano e tedesco: teoria e prassi. Italienische und deutsche Werbesprache: Theorie und Praxis. Alessandria: Edizioni dell'Orso.

De Mauro, Tullio 1967. Un linguaggio subalterno. In Baldini (1987), $51-55$.

De Stefani, Elwys 2004. La terminologia nell'onomastica. RIOn. 10/1, 53-71.

De Stefani, Elwys 2005. I marchionimi in una realtà plurilingue. Il caso della Svizzera. In Brozović-Rončević, Dunja / Caffarelli, Enzo (a c. di), Denominando il mondo. Dal nome comune al nome proprio/Naming the world. From common nouns to proper names (Quaderni internazionali di RION 1). Roma: Società Editrice Romana, 67-86.

De Stefani, Elwys 2007. Asphalttiger und Grossstadt-Gazellen. Handelsnamen als sprachliche Ressourcen in Zeitungsberichten und im Gespräch. In Kremer/Ronneberger-Sibold (2007a), 233-257.

De Stefani, Elwys 2008. L'uso dei nomi commerciali nelle conversazioni spontanee. In Arcamone (2008), 711-728.

De Stefani, Elwys 2009. Spielen Warennamen bei der Kaufentscheidung eine Rolle? Eine Untersuchung spontaner Kundeninteraktion. In Lavric, Eva (a c. di), People, products, and professions. Choosing a name, choosing a language. Frankfurt am Main: Peter Lang, 239-253.

DISC = Il Sabatini Coletti, Dizionario della Lingua Italiana. Online: $<$ dizionari.corriere.it/dizionario_italiano/> (23.7.2015).

$D L=$ Beccaria, Gian Luigi (dir.) 1996. Dizionario di linguistica e di filologia, metrica, retorica. Torino: Einaudi. 
Dufferain, Svenja 2013. Parmissimo, El Pastor de la Polvorosa, Le Montagnard des Vosges. Kontrastive Ergonymie der Kulinarik am Beispiel des 'Tyronyms'. In Eckkrammer/Thaler (2013a), 207-240.

Eckkrammer, Eva Martha / Thaler, Verena (a c. di) 2013a. Kontrastive Ergonymie. Romanistische Studien zu Produkt- und Warennamen. Berlin: Frank \& Timme.

Eckkrammer, Eva Martha / Thaler, Verena 2013b. Die Ergonymie als namenkundliche Subdisziplin. In Eckkrammer/Thaler (2013a), 7-53.

Egerland, Verner 2011. Participio. In Enciclopedia dell'Italiano. Online: <www.treccani.it/enciclopedia/participio_(Enciclope dia-dell'Italiano)/> (13.4.2015).

Eichler, Ernst / Hilty, Gerold / Löffler, Heinrich / Steger, Hugo / Zgusta, Ladislav (a c. di) 1995-1996. Namenforschung. Ein internationales Handbuch zur Onomastik = Name studies. An international handbook of onomastics $=$ Les noms propres. Manuel international d'onomastique, 2 voll. + registro (vol. $1=1995$, vol. $2=$ 1996). Berlin: de Gruyter.

Fabris, Giampaolo 2010. Prefazione. In Minestroni (2010), 7-9.

Fèvre-Pernet, Christine 2007. Onomastique commerciale et genre polysémiotique: les catalogues de jouets. Tesi dottorale, Université Toulouse-Le Mirail. Online: <tel.archives-ouvertes.fr/tel00296612/document> (18.7.2015).

Fèvre-Pernet, Christine / Roché, Michel 2005. Quel traitement lexicographique de l'onomastique commerciale? Pour une distinction Nom de marque/Nom de produit. CORELA, Numéros spéciaux: Le traitement lexicographique des noms propres. Online: <corela. revues.org/1198> (1.7.2015).

Folena, Gianfranco 1964. Aspetti della lingua contemporanea. La lingua e la pubblicità. Cultura e scuola, 9. (Ripubbl. in Chiantera 1989, 107-120).

Fowler, Geoffrey A. / Ywatani Kane, Yukari (2011). An Article of Faith for Marketers: Place No Faith in Articles. The Wall Street Journal. 12.9.2011. Online: <www.wsj.com/articles/SB1000142405 $3111903895904576546910525327024>(1.7 .2015)$. 
Gabriel, Klaus 2003. Produktonomastik. Studien zur Wortgebildetheit, Typologie und Funktionalität italienischer Produktnamen. Frankfurt am Main et al.: Peter Lang.

Gary-Prieur, Marie-Noëlle 1991. La modalisation du nom propre. Langue Française. 92, 46-63.

Gary-Prieur, Marie-Noëlle 1994. Grammaire du nom propre. Paris: Presses Universitaires de France.

Gary-Prieur, Marie-Noëlle 2005. Où il est montré que le nom propre n'est (presque) jamais «modifié». Langue Française. 146/2, 53-66.

$G D L I=$ Battaglia, Salvatore / Bàrberi Squarotti, Giorgio (a c. di) 2002. Grande Dizionario della Lingua Italiana, vol. 21: Toi-Z. Torino: UTET.

Gontijo, Possidonia F.D. / Zhang, Shi 2007. The Mental Representation of Brand Names: Are Brand Names a Class by Themselves? In Lowrey (2007), 23-37.

Grass, Thierry 2002. Quoi! Vous voulez traduire "Goethe”? Essai sur la traduction des noms propres allemand-français. Bern: Peter Lang.

Gross, Maurice 1975. Méthodes en syntaxe. Régime des constructions complétives. Paris: Hermann.

Gross, Maurice 1977. Grammaire transformationnelle du français. Syntaxe du nom. Paris: Larousse.

Grossmann, Maria / Rainer, Franz (a c. di) 2004. La formazione delle parole in italiano. Tübingen: Niemeyer.

Gutschmidt, Karl 1982. Zur Funktion der Warennamen in der Literatur. In Eichler, Ernst / Schultheis, Johannes / Walther, Hans (a c. di), Studia onomastica III. Leipzig: Karl-Marx-Universität, 20-27.

Hansack, Ernst 2004. Das Wesen des Namens. In Brendler/Brendler (2004), 51-65.

Harris, Zellig S. 1952. Discourse Analysis. Language. 28/1, 1-30.

Harweg, Roland 1983. Genuine Gattungseigennamen. In Faust, Manfred / Verburg, Pieter A. (a c. di), Allgemeine Sprachwissenschaft, Sprachtypologie und Textlinguistik. Festschrift für Peter Hartmann. Tübingen: Gunter Narr, 157-171. 
Hoegg, JoAndrea / Alba, Joseph W. 2007a. Linguistic Framing of Sensory Experience: There Is Some Accounting for Taste. In Lowrey (2007), 3-21.

Hoegg, JoAndrea / Alba, Joseph W. 2007b. Taste Perception: More than Meets the Tongue. Journal of Consumer Research. 33, 490-498. Humbley, John 2006. La traduction des noms d'institutions. Meta: journal des traducteurs/Meta: Translators'Journal. 51/4, 671-689.

INTA $(2012)=$ International Trademark Association, A Guide To Proper Trademark Use. Online: <www.inta.org/Media/Docum ents/2012_TMUseMediaInternetPublishing.pdf> (18.7.2015).

Jakobson, Roman 1956. Due aspetti del linguaggio e due tipi di afasia. In Id. (2002), Saggi di linguistica generale, cura e introduzione di Luigi Heilmann. Milano: Feltrinelli, 22-45 (1 $1^{\text {a }}$ ed. 1966; trad. dell'originale fr. Essais de linguistique générale. Paris: Éditions de Minuit, 1963).

Jakobson, Roman 1957. Shifters, Verbal Categories, and the Russian Verb. In Id. (1971), Selected Writings II. Word and Language. The Hague/Paris: Mouton, 130-147.

Jakobson, Roman 1960. Closing Statement: Linguistics and Poetics. In Sebeok, Thomas A. (a c. di), Style in Language. New York/ London: The Technology Press of Massachusetts Institute of Technology and John Wiley \& Sons, 350-377.

Janner, Maria Chiara 2012a. Sintassi dei nomi di marca in italiano. Note sulla determinazione. In Cotticelli Kurras/RonnebergerSibold/Wahl (2012), 157-175.

Janner, Maria Chiara 2012b. Le massaie di Migliorini e Federico Moccia. Sulla natura linguistica dei marchionimi in Tre metri sopra il cielo. il Nome nel testo. 14, 221-232.

Janner, Maria Chiara 2013. Notes on the syntax of commercial names in Italian. In Sjöblom/Ainiala/Hakala (2013), 250-268.

Jansen, Hanne 2011. Preposizioni. In Enciclopedia dell'Italiano. Online: <www.treccani.it/enciclopedia/preposizioni_(Enciclope dia-dell'Italiano)/> (26.10.2014).

Jonasson, Kerstin 1991. Les noms propres métaphoriques: construction et interprétation. Langue Française. 92, 64-81.

Jonasson, Kerstin 1994. Le nom propre. Constructions et interprétations. Louvain-la-Neuve: Duculot. 
Jonasson, Kerstin 1995. La camarade Catherine revisitée. In Noailly (1995), 85-94.

Jonasson, Kerstin 2005. La modification du nom propre dans une perspective contrastive. Langue Française. 146/2, 67-83.

Kalverkämper, Hartwig 1978. Textlinguistik der Eigennamen. Stuttgart: Klett-Cotta.

Kleiber, Georges 1981. Problèmes de référence: description définies et noms propres. Paris: Klincksieck.

Kleiber, Georges 1985. Sur la sémantique et pragmatique des SN Le projet Delors et La camarade Catherine. L'information grammaticale. 27, 3-9.

Kolde, Gottfried 1995. Grammatik der Eigennamen (Überblick). In Eichler et al. (1995), 400-408.

Koss, Gerhard 1996a. Warennamen. In Eichler et al. (1996), 1642-1648.

Koss, Gerhard 1996b. Warennamen-, Firmennamenrecht. In Eichler et al. (1996), 1795-1802.

Kotler, Philip 2003. Marketing insights from A to Z. 80 concepts every manager needs to know. Hoboken, N.J.: Wiley.

Kremer, Ludger / Ronneberger-Sibold, Elke (a c. di) 2007a. Names in Commerce and Industry: Past and Present. Berlin: Logos.

Kremer, Ludger / Ronneberger-Sibold, Elke 2007b. Names in Commerce and Industry: An Introduction. In Kremer/Ronneberger-Sibold (2007a), 11-21.

Kripke, Saul A. 1972. Naming and Necessity. In Davidson, Donald / Harman, Gilbert (a c. di), Semantics of natural language. Dordrecht/Boston: Reidel, 253-355.

Kuhn, Julia 2008. Strategie persuasive nella comunicazione aziendale esterna: uso degli ergonimi e strumentalizzazione della storia. In Arcamone (2008), 641-651.

La Fauci, Nunzio 2009. Compendio di sintassi italiana. Bologna: il Mulino.

La Fauci, Nunzio 2010. Nomi propri (e fatti correlati) in una telecronaca sportiva. In Pepin, Nicolas / De Stefani, Elwys (a c. di), Eigennamen in der gesprochenen Sprache. Tübingen: Francke, 200-223.

La Fauci, Nunzio 2011. Relazioni e differenze. Questioni di linguistica razionale. Palermo: Sellerio. 
LaR $=$ Corpus "La Repubblica" (annate 1985-2000). Online: <dev. sslmit.unibo.it/corpora/results.php? $\operatorname{mode}=$ advanced $\&$ path $=\&$ name $=$ Repubblica $>$ (24.9.2014).

LaR online $=$ Archivio La Repubblica (dal 1984). Online: <ricerca. repubblica.it> (24.9.2014).

Laurent, Bénédicte 2007. Le nom de marque et de produit, quand la linguistique pose aussi ses marques. In Kremer/Ronneberger-Sibold (2007a), 35-44.

Leblanc, Benoit 2008. Plagiarizing brand names: onomastics in the courtroom. Onoma. 43, 73-90.

Levi, Primo 2014. Il sistema periodico. Torino: Einaudi (1 $1^{\text {a }}$ ed. 1975).

Lindemann, Jan 2010. The Economy of Brands. New York: Palgrave Macmillan.

Lo Duca, Maria G. 2010. Complementi. In Enciclopedia dell'Italiano. Online: <www.treccani.it/enciclopedia/complementi_(Enciclope dia_dell'Italiano)/> (2.6.2015).

Lötscher, Andreas 1992². Von Ajax bis Xerox. Ein Lexikon der Produktenamen, mit einem Beitrag von Adolf Wirz. Zürich: Artemis \& Winkler (1 ${ }^{\text {a }}$ ed. 1987).

Lötscher, Andreas 1995. Der Name als lexikalische Einheit: Denotation und Konnotation. In Eichler et al. (1995), 448-457.

Lötscher, Andreas 2008. Die historischen Voraussetzungen für den Eigennamenstatus von Produktnamen. Onoma. 43, 25-56.

Lowrey, Tina M. (a c. di) 2007. Psycholinguistic Phenomena in Marketing Communications. Mahwah (New Jersey)/London: Lawrence Erlbaum Associates.

LPM = Legge federale sulla protezione dei marchi e delle indicazioni di provenienza, del 28 agosto 1992 (stato $1^{\circ}$ luglio 2011). Online: <www.admin.ch/opc/it/classified-compilation/ 19920213/201107010000/232.11.pdf > (16.1.2015).

Lury, Celia 2004. Brands. The logos of the global economy. London: Routledge.

Mangano, Dario / Marrone, Gianfranco 2015. Brand language: Methods and models of semiotic analysis. In Rossolatos (2015), 46-88.

Marrone, Gianfranco 2007. Il discorso di marca. Modelli semiotici per il branding. Roma/Bari: Laterza. 
Masson, Delphine 2000. Ces marques victimes de leur succès. Stratégies. 30.6.2000. Online: <www.strategies.fr/articles/r13573W/ ces-marques-victimes-de-leur-succes.html $>$ (18.7.2015).

Matt, Luigi 2007. Liala al tempo dei reality: i romanzi per adolescenti di Federico Moccia. Lingua Italiana d'Oggi. 4, 243-258.

Mattioda, Maria Margherita 2008. L' «Eau d'Issey» delle marche. Migrazioni lessico-culturali nell'onomastica commerciale. Synergies Italie. 4, 109-121.

Migliorini, Bruno 1927. Dal nome proprio al nome comune. Studi semantici sul mutamento dei nomi propri di persona in nomi comuni negl'idiomi romanzi. Genève: Olschki.

Migliorini, Bruno 1946. Che cos'è un vocabolario? Roma: Edizioni della Bussola.

Migliorini, Bruno 1952. Il tipo sintattico «votate socialista». Lingua nostra. 13/1, 113-118.

Migliorini, Bruno 1956. Motti pubblicitari e politici. In Id., Conversazioni sulla lingua italiana. Firenze: Le Monnier. (Ripubbl. in Chiantera 1989, 75-78).

Minestroni, Laura 2010. L'alchimia della marca. Fenomenologia di un moltiplicatore di valore. Milano: Franco Angeli.

Moccia, Federico 1992. Tre metri sopra il cielo. Roma: Il Ventaglio. (Si cita dalla ristampa Milano: Feltrinelli, 2005).

Moccia, Federico 2004. Tre metri sopra il cielo. Milano: Feltrinelli.

Molino, Jean 1982. Le nom propre dans la langue. Langages. 66, 5-20.

Muselmann, Sigrid 2010. Lebensmittelmarken in Italien. Eine sprachwissenschaftliche Untersuchung unter Berücksichtigung wirtschaftspsychologischer Aspekte. Wilhelmsfeld: Gottfried Egert Verlag.

Nicolaisen, Wilhelm F.H. 1995. Names in English Literature. In Eichler et al. (1995), 560-568.

Noailly, Michèle 1990. Le substantif épithète. Paris: Presses Universitaires de France.

Noailly, Michèle (a c. di) 1995. Nom propre et nomination. Actes du colloque de Brest, 21-24 avril 1994. Paris: Klincksieck.

Nübling, Damaris / Fahlbusch, Fabian / Heuser, Rita 2012. Namen. Eine Einführung in die Onomastik. Tübingen: Narr. 
Olsen, Lars Erling / Lanseng, Even Johan 2012. Brands in texts: Attitudinal effects of brand placements in narrative fiction. Journal of Brand Management. 19/8, 702-711.

Panunzi, Alessandro 2010. Copula. In Enciclopedia dell'Italiano. Online: <www.treccani.it/enciclopedia/copula_(Enciclopedia_ dell'Italiano)/> (28.6.2015).

Panunzi, Alessandro 2011. Predicato, tipi di. In Enciclopedia dell'Italiano. Online: <www.treccani.it/enciclopedia/tipi-di-predicato_ (Enciclopedia_dell'Italiano)/> (30.3.2015).

Petit, Gérard 2000. Un hybride sémiotique. Le nom déposé. Linguisticae Investigationes. 23/1, 161-192.

Petit, Gérard 2006. Le nom de marque déposée: nom propre, nom commun et terme. Meta: journal des traducteurs/Meta: Translators' Journal. 51/4, 690-705.

Platen, Christoph 1997. "Ökonymie”. Zur Produktnamen-Linguistik im Europäischen Binnenmarkt, Tübingen: Max Niemeyer Verlag.

Pöcksteiner, Eva 2008. "Das Markukt”. Wie eine Marke zum Begriffsmonopol wird! Dissertazione, Universität Wien. Online: <othes. univie.ac.at/8126/1/Das_Markukt.pdf $>$ (18.7.2015).

Ramaglia, Francesca 2011. Relazione, aggettivi di. In Enciclopedia dell'Italiano. Online: <www.treccani.it/enciclopedia/aggettividi-relazione_(Enciclopedia_dell'Italiano)/> (28.5.2015).

Renzi, Lorenzo 1988. L'articolo. In Renzi, Lorenzo / Cardinaletti, Anna (a c. di), Grande grammatica italiana di consultazione. Vol. I: La frase. I sintagmi nominale e preposizionale. Bologna: il Mulino, 357-423.

Rey-Debove, Josette 1995. Nom propre, lexique et dictionnaire de langue. In Noailly (1995), 107-122.

Riolo, Salvatore 2007. Marchionimi e nomi commerciali nella lessicografia italiana del secondo Novecento. Catania: CUECM.

Ronneberger-Sibold, Elke 2004. Warennamen. In Brendler/Brendler (2004), 557-603.

Ronneberger-Sibold, Elke 2007. Sprachlich hybride Markennamen im Deutschen: Ein geschichtlicher Überblick. In Kremer/Ronneberger-Sibold (2007a), 187-211. 
Ronneberger-Sibold, Elke 2008a. Die morphologische Struktur deutscher Markennamen: Diachrone Entwicklungen im Laufe des 20. Jahrhunderts. In Arcamone (2008), 777-792.

Ronneberger-Sibold, Elke 2008b. Food and learning: a twentieth century overview of educational topics in German brand names for food. Onoma. 43, 221-250.

Ronneberger-Sibold, Elke / Wahl, Sabine 2014. Associations in German Brand Names: Current Trends. In Tort i Donada/Montagut i Montagut (2014), 582-593.

Rossolatos, George (a c. di) 2015. Handbook of Brand Semiotics. Kassel: Kassel University Press.

Saussure, Ferdinand de 1916. Cours de linguistique générale, publié par Charles Bally et Albert Sechehaye. Lausanne: Payot. (Si cita dalla trad. it. a c. di Tullio De Mauro, Corso di linguistica generale. Roma/Bari: Laterza, 1997 ${ }^{13}$ ).

Saussure, Ferdinand de 2002. Écrits de linguistique générale, établis et édités par Simon Bouquet et Rudolf Engler avec la collaboration d'Antoinette Weil. Paris: Gallimard.

Schweickard, Wolfgang 1992. Deonomastik. Ableitungen auf der Basis von Eigennamen im Französischen (unter vergleichender Berücksichtigung des Italienischen, Rumänischen und Spanischen). Tübingen: Niemeyer.

Seidl, Christian 2004. Deantroponimici. In Grossmann/Rainer (2004), 409-419.

Semprini, Andrea 1992. Le marketing de la marque. Approche sémiotique. Paris: Éditions Liaisons.

Semprini, Andrea 1996. La marca. Dal prodotto al mercato, dal mercato alla società. Milano: Lupetti.

Serianni, Luca 1978. Nomi d'alberghi. Lingua nostra. 39/1, 56-62.

Shrum, L.J. / Lowrey, Tina M. 2007. Sounds Convey Meaning: The Implications of Phonetic Symbolism for Brand Name Construction. In Lowrey (2007), 39-58.

Sini, Lorella 2014. Les noms propres de marques dans quelques romans français contemporains. In Tort i Donada/Montagut i Montagut (2014), 2313-2324. 
Sjöblom, Paula / Ainiala, Terhi / Hakala, Ulla (a c. di) 2013. Names in the Economy: Cultural Prospects. Newcastle upon Tyne: Cambridge Scholars Publishing.

$\mathrm{SM}=$ Spendere Meglio. Online: $<\mathrm{www}$. consumatori.ch $>$ (24.9.2014).

Solly, Martin 2002. 'Once a trademark, not always a trademark': Using Language to Avoid Legal Controversy. In Gotti, Maurizio / Heller, Dorothee / Dossena, Marina (a c. di), Conflict and Negotiation in Specialized Texts. Selected Papers of the 2nd CERLIS Conference. Bern et al.: Peter Lang, 211-232.

Šrámek, Rudolf 1996. Namen von Sachen (Chrematonymie) I. In Eichler et al. (1996), 1562-1567.

Stim, Richard $2007^{9}$. Patent, copyright \& trademark. Berkeley: Nolo Press (1 ${ }^{\text {a }}$ ed. 1994).

Teutsch, Andreas 2007. Linguistische Aspekte der rechtlich basierten Markeneignung. Zürich: LIT Verlag.

Teutsch, Andreas 2008. Die gesetzlich regulierte Kennzeichnungspflicht von Produktemarken in Nachschlagewerken. LeGes Gesetzgebung \& Evaluation. 2, 293-304.

Thornton, Anna Maria 2004. Econimia. In Grossmann/Rainer (2004), 609-610.

Tognini-Bonelli, Elena 2001. Corpus linguistics at work. Amsterdam: Benjamins.

Tort i Donada, Joan / Montagut i Montagut, Montserrat (a c. di) 2014. Els noms en la vida quotidiana. Actes del XXIV Congrés Internacional d'ICOS sobre Ciències Onomàstiques. Annex. Generalitat de Catalunya.

Treccani $=$ Vocabolario Treccani. Online: $<$ www.treccani.it/vocabola rio/> (18.7.2015).

Tufi, Stefania / Blackwood, Robert 2010. Trademarks in the linguistic landscape: methodological and theoretical challenges in qualifying brand names in the public space. International Journal of Multilingualism. 7/3, 197-210.

Vande Casteele, An 2012. A Semantic Description of Company Names in Spanish Business-related Newspaper Articles. In Boerrigter/ Nijboer (2012), 93-103.

Vande Casteele, An 2014. Genericization of brand names in Spanish. In Tort i Donada/Montagut i Montagut (2014), 654-667. 
Van Langendonck, Willy 2007a. Theory and Typology of Proper Names, Berlin/New York: Mouton de Gruyter.

Van Langendonck, Willy 2007b. Trade and Brand Names: Proper or Common Nouns? In Kremer/Ronneberger-Sibold (2007a), 23-33.

Van Langendonck, Willy 2008. Not common nouns but proper names are the prototypical nouns. In Arcamone (2008), 815-822.

Vaxelaire, Jean-Louis 2005. Les noms propres. Une analyse lexicologique et historique. Paris: Champion.

Vincze, László 2008. Ungarische Markennamen. Onoma. 43, 115-147.

Voigt, Gerhard 1989. Zur linguistischen Bestimmung des Markennamens. In Debus, Friedhelm / Seibicke, Wilfried (a c. di), Reader zur Namenkunde. I: Namentheorie. Hildesheim/Zürich/New York: Olms, 167-180.

Wee, Lionel 2006. Proper names and the theory of metaphor. Journal of Linguistics. 42, 355-371.

Wochele, Holger / Kuhn, Julia / Stegu, Martin (a c. di) 2012. Onomastics goes business. Role and relevance of brand, company and other names in economic contexts. Berlin: Logos.

Zardo, Francesco 1996. Nomi di marchio e dizionari. Studi di lessicografia italiana. 13, 365-392.

Zardo, Francesco 1997. Marchi nominativi: nomi propri o nomi comuni? Il nome di marchio nell'italiano scritto contemporaneo. RIOn. 3/1, 25-43.

Zardo, Francesco 2007. Ancora sui marchionimi. In Della Valle, Valeria / Trifone, Pietro (a c. di), Studi linguistici per Luca Serianni. Roma: Salerno Editrice, 253-269.

Zilg, Antje 2006. Nomi di marca del settore alimentare italiano. CLAC - Círculo de Lingüística Aplicada a la Comunicación. 28, 16-34. Zingarelli $=$ AA.VV. 2014. Lo Zingarelli 2015. Vocabolario della lingua italiana. Bologna: Zanichelli. 



\section{Indice analitico}

accordo $67 \mathrm{n}, 79,124,168,244,247-248$

affisso 46, 266

prefisso 268,272

suffisso 35n, 67n, 121, 266-267

aggettivo 43-44, 50, 59, 102-103 e n, 128-130, 141, 147, 151-152, 160, 165, 183-184, 193, 198 e n, 201, 224n, 228, 239, 241-243, 263, 265-268, 277, 286, 304, 308, 314 possessivo $81,114,214,302$ qualificativo $82,102,128,130,165$, 207, 247, 263, 314

relazionale (di relazione) 147,152 , 157-158, 167, 176 e n, 182-183,

186, 193, 198, 207-210, 224, 267. 304-307, 309

ambiguità interpretativa 92, 141, 144, 148, 153-154, 173, 177, 183, 196, 199n, 216, 218, 221, 235, 239, 277, 303

antonomasia $28,43-44,49-52,106$, $114,173,183,269,271-278,280$, 282, 286 e n, 303-304

antroponimo 32n, 67 e n, 116, 142-143, $145,150,153 \mathrm{n}, 163,165,179,181$, 183-184, 202, 215, 224-225, 247n, 266, 269 e n, 307, 309

appellativo (v. nome comune) apposizione 52, 79, 90, 127-128, 132, $142,145,147,150 \mathrm{n}, 203,210$ esterna 133-135, 145-146, 202, 304-305 interna $132-133,135,144,150-151$, $182,202,277,304-305$

argomento, funzione argomentale 59-60, 77, 79, 81n, 105-109, 127, 135-137, 139, 141-142, 145, 147, 149, 151, $154,158,162-165,169-173$,
182-183, 187-188, 198, 223, 225, 237, 242-244, 256, 262-263, 273, 277, 279, 281, 286, 289-290, 294-299, 302-309, 313-314

articolo 49, 51-52, 67 e n, 79-83, 92-102, 105, 108, 110 e n, 112-115, 135-137, 162, 183, 192, 195, 199 e n, 207, 215, 219-220, 224-225, 239-240, 246, 262-263, 290 e n, 302, 306-307

determinativo 62-63, 80-81, 87-89, 92, 94, 97, 108, 113-114, 128, 149, 153, 192, 216, 218, 235-236, 259, $261,274-275,278,303-304$

indeterminativo $62,68,80,84-87$, 93-94, 97, 110n, 120, 136, 149, 157, 237-238, 244, 274, 279, 303-305

in variazione libera (v. variazione libera)

atto di battesimo 39, 42, 49, 106, 166, 305,313

attributo, funzione attributiva (v. anche modificazione e predicazione) 50, 63, 68, 77, 79, 82, 85-86, 102-105, 107n, 127-133, 140-143, 146-147, 149,153 e n, 157-160, 164-172, 183, 201, 203, 207-208, 229, 247, $267,295,302,304,314$

avverbiale, funzione $69,77,92-93,110$, 113, 136, 222, 228, 240-246, 256, 262-263, 303, 308, 315

brand awareness 28, 72, 297 brand integration 17, 301 brand placement 288, 317 categoria grammaticale $35,37-41,65$, 79, 97, 102-103 e n, 106, 214-215, 313-314 
combinatorie, proprietà (v. proprietà combinatorie)

complemento 223n, 225, 272, 278

avverbiale (v. supplemento predicativo)

di mezzo o strumento 186, 218, 307

di modo o maniera $222 \mathrm{n}$

di specificazione 83-84, 142, 162 , 166-167, 176, 185-186, 193-197, 205, 207, 210, 214, 278-279, 307

predicativo del soggetto/dell'oggetto 222, 255

preposizionale del predicato 185 ,

188-192, 223, 307

composizione 268-269

connotativo/denotativo 30, 32, 40, 42, 45, 48-49, 106, 288, 293

consumatore $17,24,26-28,30-31,43$, $45,297 \mathrm{n}$

copula (v. costruzione copulativa)

co-referenza 90 e n, 98, 100, 140, 150, 237

corpus $51,70-72,312$

correlazione $60,83,97,136,186,230$, 262-263, 302-303

costrutto (v. costruzione)

costruzione

copulativa (o copulare) 91, 145, 153, 227-228, 236, 238, 247, 255, 263, 285 identificativa 147, 236-237, 239, 308 passiva $185,187-188,223,225,228$, 230n, 231n, 240, 244, 248 e n, 307 denominazione, denominativo 29 , 39-40, 46, 102, 105-106, 165, 179, $247,250-251,289$

deonomastica 41, 265 e n, 267

derivazione 67n, 207, 265-266, 269

determinazione, determinatore

(v. articolo)

diacronia, diacronico 45, 46n, 48, 100, 113, 159n, 216, 240-242, 288, 295, 315

diatopia, diatopico 87, 123n, 213-214 e n, 224, 259n, 316 discretezza 64-65, 124

diritto, aspetti giuridici 24-25, 26n, 28-30, 35-36, 39, 41-43, 46-49, $66,113,157,161 \mathrm{n}, 165$

distribuzione, distribuzionale 17, 168, 234, 301, 315

dizionario 28, 37, 39, 41, 43 e n, 47-50, $64,109,119$ n, 173, 189, 281, 286 e n economia, economico 24, 27-29, 31, 34-36, 43, 157, 195, 288, 316-317 ellissi 43, 68, 80, 98-101, 105, 107, 121, $123,136,295,306,313$

enciclopedia, conoscenze enciclopediche 64, 95, 109n, 110, 143, 151, 160, 177, 290

etichetta $24 n, 32,42,44 n, 50,96,105$, $165,169,181,243,246-251$

etimologia 33n, 46, 53

fonetica, fonetico $31-32$

frazionante, uso $67,85,149,152$

frequenza $65,72,87,101,121-122$, 143, 151, 183, 189, 195, 198, 206n, 214n, 228, 248, 256, 263, 307

funzione $18,59,97,102-103,135-136$, $225,265,306,311,314$ genere 67 e $\mathrm{n}, 68,79-80,97-101$, 116-124, 131n, 136, 144, 146, 251n, 303, 306, 313, 316

genericizzazione 41-44, 49-50, 52, 115 , 316

grafia, grafico 27 e n, 42-43, 48, 61, 155-156, 160n, 272, 305

interpretazione, interpretativo 16,47 , 63-64, 85-86, 99 e n, 141, 143, 177 e n, 191, 223n, 225, 245-246, 269, 276, 309

invarianza formale $17,102,151,201$, $243,265,306,311$

legittimare, legittimazione 53, 69, 87, $89,141,185,187-189,193,196$, 213, 223, 228-231, 233, 235, 242, 258, 261-262, 291, 302, 305, 308

lessicalizzazione (v. anche genericizzazione) 42-44, 278, 286n 
lessico 28, 60

lessicografia 43n, 47-50, 60, 119n, 286, 290, 316

locativo (v. supplemento predicativo locativo)

logo 155-156, 305

luogo comune 28, 47-48, 79, 105-106, $114,116,136,154,172-174,293$, 295-299, 305-306, 313

marcato, marcatezza 94, 105, 112-116, 119-121, 123 e n, 272, 290, 302-303

marcatezza interpretativa $62,65,80,87$, $113,142,231,233,240,244$

marchio registrato $30-31,42,47 \mathrm{n}$, 48-49, 109, 115, 131, 200, 222

marketing 24n, 27-32, 33n, 36, 41, 46n, 164-165, 297, 316

metafora, metaforico $37 \mathrm{n}, 68,82 \mathrm{n}$, 182n, 225, 231-232, 250-253, 255-256, 267, 269 e n, 271-287, 305,317

metalingua, metalinguistico 39-42, 49, 104-106, 123, 152, 154, 164, 166, $174,228-229,246-247,271-272$

metonimia, metonimico 37 e n, 42-44, 49-50, 66-68 e n, 89, 97, 100, 164, $169,195,206$ n, 222, 229-232, 254, 260-262, 264, 283-284, 290n, 294, $297,302,305,309,313$

modalizzante, uso $68,85-86,304$ modificazione (v. anche apposizione e attributo) 69, 79, 81, 127, 193, 210, 222-223, 277, 306-307

attributiva (non-denominativa) 77 , 115n, 129-130, 133, 143, 157, $165-169,172$

denominativa $77,104,133,135$, 140-144, 148-153, 163, 166, 172n, 181-182, 248-250, 304-305, 308, 313-314

restrittiva/non-restrittiva (o descrittiva) 84-86, 129-130, 132, 150, 152, 204, 304 morfologia, morfologico 45-46, 67, 102, 121-122, 126 e n, 198n, 227, 263, $265,288 \mathrm{n}, 311$

naming, creazione dei nomi 29-32, 45-46 e $n$

nome comune 37-42, 44n, 46n, 47, $50,53,68,77,79-80,90,94,96$, 98-99, 101, 103-107, 114-116, 126, 132-134, 137, 139-152, 164-166, 171-173, 175-177, 179, 182-184, 193-194, 198-200, 204, 211, 214-215, 218, 224, 228n, 243, 268, 272, 280, 286n, 291, 296-297, 305-306, 311, 313-314

nome di luogo (v. toponimo) nome di persona (v. antroponimo) nome proprio $16-18,32 \mathrm{n}, 35 \mathrm{n}, 37-41$, 43-44, 46n, 48, 57, 64-69, 72, 79, $85,87,96,99,101-102,105-106$, $109,114,128,133,140-143$, 149-151, 166, 177 e n, 182-183, 198n, 201, 215, 227n, 269 e n, 271-272, 276, 278, 286, 294, 296, 304, 313-314

prototipico 46n, 67-68 e n, 85, 103n, $112,116,137,139,143,149,155 \mathrm{n}$, $177,182,184,224$

nominale, predicato (v. predicato nominale)

nominalizzazione 101-105, 131, 135-137, 146, 171-173, 182, 190n, 306,313

nucleo (del sintagma o nesso nominale) $59,77,79,133$, 140-141, 143-144, 146, 149-151, 154, 171, 183, 204, 206, 210, 272-273, 302, 307, 309 numero 43, 67 e n, 79-80, 81n, 92-95, 97-100, 110 e n, 112, 116, 119 , 121-122, 124-127, 136, 146, 148, 169n, 202, 204, 214, 235, 256, 274-276, 290n, 302-304, 306, 308-309, 313 
oggetto

diretto $69,91,92,94,113,120 \mathrm{n}, 136$, 195-196, 198, 227-228, 232-235, 240, 242-244, 262, 303, 308

indiretto $92,187-188,223,227,258$, 307

omonimia $17,30,66,72,115$

onomastica 16, 32n, 33-36, 40, 45, $52-54,79,288,315$

opposizione $18,58,60,63-64,67,87$, 109-110, 116, 119, 123-124, 129, 136, 211 e n, 245, 293, 312

paradigmatico (v. rapporto sintagmatico/paradigmatico)

parafrasi 68, 104, 142, 178, 267

passivo (v. costruzione passiva)

patronimico 32, 66, 103n, 133, 163, $247 \mathrm{n}, 248$

personificazione $89,116,291$

pertinenza 18, 62, 97-98, 101, 107-109, $116,122,136,261,276,302$

plagio 30

plurale (v. numero)

possesso, possessivo (v. anche aggettivo) $148,165,175,178,180,208,232$, 305,307

predicato, funzione predicativa 18,50 , 59-60, 77, 103, 105, 107, 127, 133-137, 139, 141-142, 152, 160, 163, 169-173, 182-183, 185, 188, 193-195, 198, 209-211, 222, 228, 235-236, 238-239, 243-244, 247, 250, 263, 267, 269, 277, 281-283, 286, 289-291, 295, 304, 306-308, 313-316

nominale 227-228, 236, 238-239, 251-253, 255, 263, 272, 275-276, 308

verbale $69,89,93,161,186,193,223$, 225, 228-231, 233-234, 240, 242, 256, 258-261, 263, 291, 302, 305, 308-309

predicato di denominazione 102, 105, 142-144, 166, 171, 304 prefisso (v. affisso)

preposizione, complemento

preposizionale $63,69 \mathrm{n}, 81,110-112$,

$131,142,147,150,153$ e n, 158, 160-161, 167-168, 175, 178, 183-226, 248n, 256-260, 284-285, 290 e n, 302, 305-307, 315

processo $17-18,23,41,57,60-61,97$, 98, 100-101, 105, 107, 136-137, 250, 306, 313-314, 316

prodotto $24-35,37-44,46,49-50,66$, 79-80, 91, 95-97, 101, 106, 107n, 108-110, 113-114, 132, 142, 161n, 164-169, 173, 182n, 186, 195, 198-201, 206 e n, 211, 218, 224, 232, 234-235, 243n, 248-251, 263, 285, 289, 291-292, 294, 298, 300, 303, 305-307, 312

produttore $24-27,41 \mathrm{n}, 42,80,103 \mathrm{n}$, 132, 142, 165, 173, 186, 193, 198, 201, 224, 232, 243n, 305, 307

proprietà combinatorie $17,58,80$, 96-97, 119, 128, 135-136, 141, 143, 146-147, 152-153, 155-156, 158, 166, 175, 184, 193, 206-207, 215, 224, 227-228, 235, 244, 301, 312,314

protezione giuridica 29-31, 41

prototipo, prototipico $19,71,173,179$, 271, 273-274, 279, 286, 315

pubblicità $28,43,46,52,70,93,107 \mathrm{n}$, $115,140,166,169,191 \mathrm{n}, 202$, 238n, 239-243, 245-246, 253, 263, 265, 269, 273, 287n, 288, 293, 301, 315

qualificativo (v. aggettivo qualificativo) rapporto sintagmatico/paradigmatico 16-17, 57-58, 60-61, 81, 93, 135-136, 146-147, 312 referente 36-40, 42, 52, 61, 63-64, 66, 79, 96-97, 136, 142, 163, 177, 184, 246, 269, 271-272, 278, 282, 285, 289, 291, 294-295, 312

relativa (v. subordinata relativa) 
relazionale (v. aggettivo relazionale) relazione di possesso (v. possesso) restrizioni di selezione $89,194-195$, 228-231, 305, 308

ridondanza 99-100, 151-152, 164, 174-175, 183, 255, 291, 296-298, 313

semantica, semantico 18, 30-32, 41-42, $47,51,53,61,63-65,69,77,96$, 101, 124, 136, 140n, 150-151, 172, 193, 204, 222n, 223n, 225, 228, 234, 272, 293

sintagmatico (v. rapporto sintagmatico/ paradigmatico)

sistema, sistemico 18, 57-58, 60-61, 78, $81,112,116,124,135,264,288$, 291-294, 300

soggetto 53, 62, 69, 87, 89, 91, 120n, 140-141, 153, 178, 191, 194, 196, 198, 218, 227-233, 235, 237-240, 256, 258 e n, 260-264, 276, 291 , 302-303, 305, 308-309

grammaticale 240,307

nozionale o logico 185, 187-188, 196, $198,223,225$

spazio, spaziale $84,132,278-279$

subordinata relativa $82-83,85,93,147$, 247,260

suffisso (v. affisso)

supplemento predicativo

locativo 186, 192, 212-218, 220, 223-225, 261-262, 307

strumentale o modale 186, 222, 307 tempo, temporale $84,132,207,268$, 294-296, 298-300

toponimo $32 \mathrm{n}, 85 \mathrm{n}, 130,199 \mathrm{n}, 261$, 264,272

traslato, uso $122,162-163,180 \mathrm{n}, 211$, $219,249,263,271,286,305$

umano/non-umano, tratto 53, 65, 69, 89, 94, 104, 120n, 142-143, 164-165, 179-181, 186, 188, 193, 195, 201, 202 e n, 204, 224, 228, 230-232, 254, 260-262, 264, 268, 291, 302, $305,307-309$

valore $16-17,57-58,61-63,67,85 n$, 89, 92, 94, 97, 101, 106, 112-113, 146, 173, 233, 235-236, 288, 295, 302-305, 307-308, 312-314

valore negativo 58,345

variazione libera

del genere 116-118, 131n, 303

del numero 127, 303

dell'articolo 51, 62-63, 67, 80, 87, 89, 91-92, 94-95, 109, 112-114, 120, $136,142,152,160-161,183,187$, 190, 204, 206, 210, 219, 224-225, 239-240, 262-263, 302-304, 306-307, 313, 315

della preposizione $d i 63,112,142$, 147, 166-167, 176, 193, 197, 201, 207-210, 224, 304-305

verba dicendi 229-230, 257, 260-261, 308

verbo supporto $69,228,234,245,256$

volgarizzazione (v. genericizzazione) 



\section{Indice dei nomi di marca citati}

Acqua Panna 101-102, 107

Adidas 59, 82, 88, 93, 95, 128, 168, 173, 176, 181, 189 e n, 194, 196, 199-201, 239, 245, 254n, 256, 274, 290-291

Adidas Gazelle 131

AEG 238

Agip 208, 215, 241-242

Agnesi 235

Aia 133, 199n

Aiwa 144, 151-152

Aldi 214

Alfa Romeo 61-62, 98, 103, 115, 128, $153,155,161,168,174,192,194$, 196, 201-202, 216-217, 229, 230n, $231,236,238,266,268$

Alfa Romeo “ 155 ” 298

Alfa Romeo "164” 292

Algida 231, 249

Alitalia 92, 104, 156, 172, 179, 187-188, 194, 201, 208-209, 217, 241-242, 252

Alleluya 166, 169

Apple 88, 103-104, 180, 194, 199-200, 208, 252, 259

Aprilia 169, 176, 203, 221, 237, 258

Armani 15, 93, 104, 153, 247-248

Aspirina 49n, 126, 206, 233-234, 276

Auchan 231, 314

Audi 236

Autogrill 195

Avirex 295

AZ 165

Baci Perugina 211, 233, 273, 281-282, 284-285

Badoit 107

Bally 159
Barbie 82-83, 91 e n, 127-128, 132, 194, 211, 271-272, 275-279, 283, 285-286 e n

Barilla 103, 130, 139, 144, 155-156, 159, 167, 181, 196, 209, 216, 231-232, 242, 246, 257-259, 268

Bassetti 249

Bata 160

Bayer 262

Benetton 90, 151, 155-156, 197, 198n, 242, 266, 293 e n, 304

Bennet 214

Beretta 159, 192

Bertolli 232

Bianchi 154, 244

Biotherm 88

Birkenstock 106, 117, 125, 134, 222

Blackberry 211

BMW 110, 220, 232, 238, 250

Bosch 109, 253

Boxer 294

Brahma 154

Bratz 91

Breil 159

Buitoni 168, 244, 248n, 282

Burberry 221

Cablecom 229

Cadbury Schweppes 196

Callaway Golf 189

Calvin Klein 199

Camay 165

Camel 294

Camomilla 199n, 289-290

Campari 209

Canon 104

Carapelli 234

Carrefour 176 e n, 204, 214

Chivas Regal 15 
Chloé 244

Chrysler 190

Cirio 83

Citroën 80n, 84, 124-125, 268

Clark's 98, 292

Clearasil 165

Coca-Cola 26n, 31n, 42n, 72, 111, 156, $187,205,233,244,273-275$

Coccoina 111

Codemi 133, 144

Coin 188

Colgate 198, 210

Continental 90, 238

Converse All Star 131

Converse One Star 131

Coop 88, 108, 114, 132, 161, 167,

176 e n, 201-203, 213-214 e n, 229, 247, 249

Coop Prix Garantie 108

Credito Varesino 89n, 120n

Daewoo 154

Dainese 159

Dash 200, 206-207

De Agostini 139

De Cecco 267

Delhaize 204

Denner 108, 213, 316

Diadora 217, 260

Diamond-Star Motors 190

Dixan 200

Dr Marten's 298

Ducati 221

Dunlop 178

Dyson 109

Edison 201, 231n

El Charro 254

Electrolux 109

Emilio Pucci 189

ENI 132, 147

Ericsson 252

Eridania 188, 234

Evian 108, 205

Facco 133

Fender 112, 292
Fendi 35

Ferrarelle 103, 107, 145, 170-171, 190, 196, 205, 208, 218, 231n, 233, 235

Ferrari 87, 98, 130, 162, 238, 275

Ferrero 167, 215, 238

Ferruzzi 235

Fiat 42, 52, 61-63, 67n, 68, 81 e n, 83-86, 89, 98, 102-103 e n, 113, $121,128-129,132,139,146-149$, 151, 153-154, 158, 161-162, 169n, 172-179, 181, 187, 189-190 e n, 191n, 192, 194, 197, 202n, 203-204, 207-208, 212, 215, 218, $230,232,235,237-239,247$, 250-255, 257-260, 277

Fiat Barchetta 95

Fiat Cinquecento 275, 289

Fiat Punto 102, 289

Fiat Tipo 139

Fiat Uno 128

Fidel 249

Findus 239

Fini 159

Fininvest 181

Fiorucci 213

Fischer 145

Fisia 147

Fiuggi 101

Föhn 42

Folletto 28

Ford 66, 72, 79-80 e n, 83-84, 89, 106n, $154 \mathrm{n}, 163-164$ e n, 236-237

Ford Fiesta 255

Ford Transit 117-118

Free 294

Frigidaire 42 e $n$

Fruit of the Loom 95

Fuji 100, 231

Fujitsu 144

Galbani 87, 196

Garofalo 267

Gaudianello 107

Generali 121, 252

General Motors, GM 157, 187, 273 
Geox 249

Gilera 221

Gillette 53, 158

Goodyear 90, 133, 203

Google 252

GORE-TEX 222

GS 249

Gucci 157, 160 e n

Guinness 84, 94, 120, 134

Guzzini 246

Harley-Davidson 97, 111, 219

Head 145, 256

Heineken 99

Henkel 200

Henniez 202

Hilton 107

Honda 102, 160, 192, 258n

Hoover 42, 109

IBM 15

Ikea 212-213

Invicta 93, 95, 148, 153

Iper 214

iPad 50n, 252

iPod 50n, 200

Iveco 146-148, 199-200, 251-252 e n

Jacuzzi 117, 130, 172, 234, 277

Jaguar 255

John Deere 250

Kellogg's 109n

Kinder 292

Kindle 252

Kleenex 182n, 205, 219, 280

Knirps 42

Kodak 31n, 88, 132, 180, 188, 202n

Kraft 200

Krupp 238

K-way 41

Lacoste 48, 86, 104, 111, 170-171, 221, 249

Lamborghini 98

Lambretta 83, 115, 126 e n, 219, 268

Lancia 15, 82, 153, 169, 174, 201, 218

Lancia Dedra 131

Lancia Delta 81n, 232, 266
Lancia Thema 15, 219

Land Rover 232

Lavazza 83n, 103 e n, 111, 134, 139, 145, 197, 205, 208-209, 230 e n, 243, 257-258

Legnano 244

Lego 127, 129, 209-210

Lepetit 192

Levissima 107

Levi Strauss, Levi's 93, 124-125, 129, 197, 205, 273, 289

Lexus 154

Liebig 43, 296

Lines 90, 122, 152

L'Oréal 133

Louis Vuitton 199-200

Luxottica 129, 273

Macintosh, Mac 101, 208

Maggi 161

Maizena 114

Marelli 189

Märklin 238

Marlboro 125, 134, 139, 156-157, 159-161, 205, 206n, 243, 246, 253, 289,294

Mars 132

Maserati 98, 191, 221

Matsushita 90

McDonald's 93, 210, 212, 238, 243, 267, 272, 274

McLaren 180

Mediolanum 191n

Melisa 213

Mercedes 122-123 e n, 179, 195, 220, 233, 238, 250, 289, 291

Metzeler 153, 159

Michelin 170n, 207, 215, 217, 257, 281

Microsoft 180, 251

Miele 109, 238

Migros 84, 89, 108, 114, 132, 180, 204, $213,214 n, 230$

Migros M-Budget 108, 169-170

Migros Sun Look 153

Miostar 109 
Missoni 189, 199n, 249

Miss Sixty 295

Mitsubishi 160

Mivit 168

Moncler 98, 109n, 118, 254

Mondadori 143, 190-191, 215-216, 258

Monopoli 193

Montano 144

Montedison 121, 146, 162, 189

Moschino 154

Moto Guzzi 98, 117, 128, 145, 187, 219-221, 259, 277

Mulino Bianco 200n

MV 221

Naj Oleari 254

Nepi 107

Nestlé 24n, 132, 217, 244, 274

Nike 93, 110 e n, 129, 134, 168, 187, 189 e n, 216-217, 242, 249, 252, 273-274, 290

Nikon 50 e n, 104

Nissan 90, 201, 229

Nissan Micra 289

Nivea 96n, 155

Nutella 115 e n, 182n, 209, 279

Nylon 42, 48

Olivetti 129, 143, 149, 188, 247

Onyx 200n

Opel 229

Opel Astra 116

Pampers 90, 122, 161, 165, 172, 182, 195

Panasonic 109

Panzani 235

Parmacotto 173

Parmalat 83

Pavesi 196

Pavesini 188

Pepsi, PepsiCo 26n, 94, 134, 187, 230, 244, 248, 273-274

Peroni 68

Perrier 107, 176

Persol 152

Perugina 255, 281

Peugeot 49n, 103n, 119, 180, 232
Philip Morris 157, 249

Philips 109, 167

Piaggio 83, 131, 149, 244

Pioneer 15, 289

Pirelli 90, 153, 159, 178, 180, 191, 242-243 e n, 251-252, 257

Plasmon 202 e n, 285 e n

Playstation 211

Porsche 51, 110

Post-it 286n

Prada 68n, 133, 248, 252, 255

Procter \& Gamble 200

Puma 131, 189n

RAI 248, 253, 261

Ralph Lauren 156, 251, 290

Ratti 90

Ray-Ban 98, 107, 110, 124, 128, 170-171, 289, 294

Ray-Ban Balorama 294

Reebok 93, 134, 222, 256

Renault 79, 80n, 89, 174, 178, 218, 229-230, 237, 257, 259

Repetto 167

Revlon 198

Rimmel 41n, 205n

Roberto Cavalli 140

Rolex 15, 95, 125, 181, 195, 211n

Rolls-Royce 145

Rotel 109

Samsung 82, 130

San Benedetto 107

San Faustino 107

Sangemini 108

San Pellegrino 30, 107

Sanpellegrino 30

Santagostino 172

Satrap 109

Schwarzkopf 133, 259n

Scotch 41, 49n, 265n

Scottex 28, 119n, 276

SEA 236

Seagram 198

Seat 191

Shell 102 
Soda-Club 167

Sofficini 126

Sony 88-90, 109n, 111, 120, 129, 144, $160,168,198,237,249$

Sony Ericsson 259n

Splendid 165

Standard SR 109

Stefanel 90

Superga 62, 111, 124-125, 130, 222, 243 e n, 256

Superquinn 204

Suzuki Vitara 131 e n, 170

Swatch 146, 167, 199-200 e n, 204, 211 , $224 n, 255,269$ e $n$

Tarzan 52

Tavernello 95

Tefal 164

Tempo 42

Thomson 192

Thyssen 238

Timberland 93, 98, 108, 111, 130, 222, $254,283,313$

Tobacco 298

Toblerone 130

Toshiba 50, 170-171

Toyota 90, 195
Trisa 109

Trudi 159

Tupperware 213

Uliveto 101

Valentino 15, 153n, 189, 199n

Valser 169

Vera 101

Versace 240

Vespa 83, 98, 115, 121, 126 e n, 131, 219,266

Victors 165

Vittel 107

Voiello 280

Volkswagen 80 e n, 90, 121, 180, 230, 236

Volkswagen Golf 292

Volkswagen New Beetle 289

Volvo 195

Walkman 49, 286n

Weber 109

Wrangler 155

Wuber 145

Xerox 43-44, 198, 282

Yamaha 118 e n, 167, 187, 218, 220

Yomo 107n, 166, 262, 268

Yuasa 144

Zyliss 168 

'[...] I might find somewhere where I can finish my book. I have thought of a nice ending for it: and he lived happily ever after to the end of his days.'

Gandalf laughed. 'I hope he will. But nobody will read the book, however it ends.'

'Oh, they may, in years to come. [...]'

J.R.R. Tolkien, The Fellowship of the Ring 



\section{Linguistic Insights}

\section{Studies in Language and Communication}

This series aims to promote specialist language studies in the fields of linguistic theory and applied linguistics, by publishing volumes that focus on specific aspects of language use in one or several languages and provide valuable insights into language and communication research. A cross-disciplinary approach is favoured and most European languages are accepted.

The series includes two types of books:

- Monographs - featuring in-depth studies on special aspects of language theory, language analysis or language teaching.

- Collected papers - assembling papers from workshops, conferences or symposia.

Each volume of the series is subjected to a double peer-reviewing process.

Vol. 1 Maurizio Gotti \& Marina Dossena (eds)

Modality in Specialized Texts. Selected Papers of the $1^{\text {st }}$ CERLIS Conference.

421 pages. 2001. ISBN 3-906767-10-8 - US-ISBN 0-8204-5340-4

Vol. 2 Giuseppina Cortese \& Philip Riley (eds)

Domain-specific English. Textual Practices across Communities and Classrooms.

420 pages. 2002. ISBN 3-906768-98-8 · US-ISBN 0-8204-5884-8

Vol. 3 Maurizio Gotti, Dorothee Heller \& Marina Dossena (eds)

Conflict and Negotiation in Specialized Texts. Selected Papers

of the $2^{\text {nd }}$ CERLIS Conference.

470 pages. 2002. ISBN 3-906769-12-7 · US-ISBN 0-8204-5887-2

Vol. 4 Maurizio Gotti, Marina Dossena, Richard Dury, Roberta Facchinetti \& Maria Lima

Variation in Central Modals. A Repertoire of Forms and Types of Usage

in Middle English and Early Modern English.

364 pages. 2002. ISBN 3-906769-84-4 · US-ISBN 0-8204-5898-8

\section{Editorial address:}

Prof. Maurizio Gotti Università di Bergamo, Dipartimento di Lingue, Letterature Straniere e Comunicazione, Piazza Rosate 2, 24129 Bergamo, Italy Fax: +39 035 2052789, E-Mail: m.gotti@unibg.it 
Vol. 5 Stefania Nuccorini (ed.)

Phrases and Phraseology. Data and Descriptions.

187 pages. 2002. ISBN 3-906770-08-7 . US-ISBN 0-8204-5933-X

Vol. 6 Vijay Bhatia, Christopher N. Candlin \& Maurizio Gotti (eds)

Legal Discourse in Multilingual and Multicultural Contexts.

Arbitration Texts in Europe.

385 pages. 2003. ISBN 3-906770-85-0 - US-ISBN 0-8204-6254-3

Vol. 7 Marina Dossena \& Charles Jones (eds)

Insights into Late Modern English. $2^{\text {nd }}$ edition.

378 pages. 2003, 2007.

ISBN 978-3-03911-257-9 • US-ISBN 978-0-8204-8927-8

Vol. 8 Maurizio Gotti

Specialized Discourse. Linguistic Features and Changing Conventions.

351 pages. 2003, 2005.

ISBN 3-03910-606-6 · US-ISBN 0-8204-7000-7

Vol. 9 Alan Partington, John Morley \& Louann Haarman (eds)

Corpora and Discourse.

420 pages. 2004. ISBN 3-03910-026-2 • US-ISBN 0-8204-6262-4

Vol. 10 Martina Möllering

The Acquisition of German Modal Particles. A Corpus-Based Approach. 290 pages. 2004. ISBN 3-03910-043-2 - US-ISBN 0-8204-6273-X

Vol. 11 David Hart (ed.)

English Modality in Context. Diachronic Perspectives.

261 pages. 2003. ISBN 3-03910-046-7 . US-ISBN 0-8204-6852-5

Vol. 12 Wendy Swanson

Modes of Co-reference as an Indicator of Genre.

430 pages. 2003. ISBN 3-03910-052-1 - US-ISBN 0-8204-6855-X

Vol. 13 Gina Poncini

Discursive Strategies in Multicultural Business Meetings.

$2^{\text {nd }}$ edition. 338 pages. 2004, 2007.

ISBN 978-3-03911-296-8 · US-ISBN 978-0-8204-8937-7

Vol. 14 Christopher N. Candlin \& Maurizio Gotti (eds)

Intercultural Aspects of Specialized Communication.

$2^{\text {nd }}$ edition. 369 pages. 2004, 2007.

ISBN 978-3-03911-258-6 · US-ISBN 978-0-8204-8926-1

Vol. 15 Gabriella Del Lungo Camiciotti \& Elena Tognini Bonelli (eds)

Academic Discourse. New Insights into Evaluation.

234 pages. 2004. ISBN 3-03910-353-9 . US-ISBN 0-8204-7016-3

Vol. 16 Marina Dossena \& Roger Lass (eds)

Methods and Data in English Historical Dialectology.

405 pages. 2004. ISBN 3-03910-362-8 · US-ISBN 0-8204-7018-X

Vol. 17 Judy Noguchi

The Science Review Article. An Opportune Genre in

the Construction of Science.

274 pages. 2006. ISBN 3-03910-426-8 · US-ISBN 0-8204-7034-1 
Vol. 18 Giuseppina Cortese \& Anna Duszak (eds)

Identity, Community, Discourse. English in Intercultural Settings.

495 pages. 2005. ISBN 3-03910-632-5 - US-ISBN 0-8204-7163-1

Vol. 19 Anna Trosborg \& Poul Erik Flyvholm Jørgensen (eds)

Business Discourse. Texts and Contexts.

250 pages. 2005. ISBN 3-03910-606-6 · US-ISBN 0-8204-7000-7

Vol. 20 Christopher Williams

Tradition and Change in Legal English. Verbal Constructions

in Prescriptive Texts.

$2^{\text {nd }}$ revised edition. 216 pages. 2005, 2007. ISBN 978-3-03911-444-3.

Vol. 21 Katarzyna Dziubalska-Kolaczyk \& Joanna Przedlacka (eds)

English Pronunciation Models: A Changing Scene.

$2^{\text {nd }}$ edition. 476 pages. 2005, 2008. ISBN 978-3-03911-682-9.

Vol. 22 Christián Abello-Contesse, Rubén Chacón-Beltrán,

M. Dolores López-Jiménez \& M. Mar Torreblanca-López (eds)

Age in L2 Acquisition and Teaching.

214 pages. 2006. ISBN 3-03910-668-6 · US-ISBN 0-8204-7174-7

Vol. 23 Vijay K. Bhatia, Maurizio Gotti, Jan Engberg \& Dorothee Heller (eds)

Vagueness in Normative Téxts.

474 pages. 2005. ISBN 3-03910-653-8 · US-ISBN 0-8204-7169-0

Vol. 24 Paul Gillaerts \& Maurizio Gotti (eds)

Genre Variation in Business Letters. $2^{\text {nd }}$ printing.

407 pages. 2008. ISBN 978-3-03911-681-2.

Vol. 25 Ana María Hornero, María José Luzón \& Silvia Murillo (eds)

Corpus Linguistics. Applications for the Study of English.

$2^{\text {nd }}$ printing. 526 pages. 2006, 2008. ISBN 978-3-03911-726-0

Vol. 26 J. Lachlan Mackenzie \& María de los Ángeles Gómez-González (eds)

Studies in Functional Discourse Grammar.

259 pages. 2005. ISBN 3-03910-696-1 - US-ISBN 0-8204-7558-0

Vol. 27 Debbie G. E. Ho

Classroom Talk. Exploring the Sociocultural Structure of Formal ESL Learning.

$2^{\text {nd }}$ edition. 254 pages. 2006, 2007. ISBN 978-3-03911-434-4

Vol. 28 Javier Pérez-Guerra, Dolores González-Álvarez, Jorge L. Bueno-Alonso

\& Esperanza Rama-Martínez (eds)

'Of Varying Language and Opposing Creed'. New Insights into Late Modern English. 455 pages. 2007. ISBN 978-3-03910-788-9

Vol. 29 Francesca Bargiela-Chiappini \& Maurizio Gotti (eds)

Asian Business Discourse(s).

350 pages. 2005. ISBN 3-03910-804-2 · US-ISBN 0-8204-7574-2

Vol. 30 Nicholas Brownlees (ed.)

News Discourse in Early Modern Britain. Selected Papers of CHINED 2004.

300 pages. 2006. ISBN 3-03910-805-0 - US-ISBN 0-8204-8025-8

Vol. 31 Roberta Facchinetti \& Matti Rissanen (eds)

Corpus-based Studies of Diachronic English.

300 pages. 2006. ISBN 3-03910-851-4 · US-ISBN 0-8204-8040-1 
Vol. 32 Marina Dossena \& Susan M. Fitzmaurice (eds)

Business and Official Correspondence. Historical Investigations. 209 pages. 2006. ISBN 3-03910-880-8 · US-ISBN 0-8204-8352-4

Vol. 33 Giuliana Garzone \& Srikant Sarangi (eds)

Discourse, Ideology and Specialized Communication.

494 pages. 2007. ISBN 978-3-03910-888-6

Vol. 34 Giuliana Garzone \& Cornelia Ilie (eds)

The Use of English in Institutional and Business Settings.

An Intercultural Perspective.

372 pages. 2007. ISBN 978-3-03910-889-3

Vol. 35 Vijay K. Bhatia \& Maurizio Gotti (eds)

Explorations in Specialized Genres.

316 pages. 2006. ISBN 3-03910-995-2 - US-ISBN 0-8204-8372-9

Vol. 36 Heribert Picht (ed.)

Modern Approaches to Terminological Theories and Applications. 432 pages. 2006. ISBN 3-03911-156-6 - US-ISBN 0-8204-8380-X

Vol. 37 Anne Wagner \& Sophie Cacciaguidi-Fahy (eds)

Legal Language and the Search for Clarity / Le langage juridique et la quête de clarté.

Practice and Tools / Pratiques et instruments.

487 pages. 2006. ISBN 3-03911-169-8 · US-ISBN 0-8204-8388-5

Vol. 38 Juan Carlos Palmer-Silveira, Miguel F. Ruiz-Garrido \&

Inmaculada Fortanet-Gómez (eds)

Intercultural and International Business Communication.

Theory, Research and Teaching.

$2^{\text {nd }}$ edition. 343 pages. 2006, 2008. ISBN 978-3-03911-680-5

Vol. 39 Christiane Dalton-Puffer, Dieter Kastovsky, Nikolaus Ritt \&

Herbert Schendl (eds)

Syntax, Style and Grammatical Norms. English from 1500-2000.

250 pages. 2006. ISBN 3-03911-181-7 · US-ISBN 0-8204-8394-X

Vol. 40 Marina Dossena \& Irma Taavitsainen (eds)

Diachronic Perspectives on Domain-Specific English.

280 pages. 2006. ISBN 3-03910-176-0 · US-ISBN 0-8204-8391-5

Vol. 41 John Flowerdew \& Maurizio Gotti (eds)

Studies in Specialized Discourse.

293 pages. 2006. ISBN 3-03911-178-7

Vol. 42 Ken Hyland \& Marina Bondi (eds)

Academic Discourse Across Disciplines.

320 pages. 2006. ISBN 3-03911-183-3 · US-ISBN 0-8204-8396-6

Vol. 43 Paul Gillaerts \& Philip Shaw (eds)

The Map and the Landscape. Norms and Practices in Genre.

256 pages. 2006. ISBN 3-03911-182-5 - US-ISBN 0-8204-8395-4

Vol. 44 Maurizio Gotti \& Davide Giannoni (eds)

New Trends in Specialized Discourse Analysis.

301 pages. 2006. ISBN 3-03911-184-1 . US-ISBN 0-8204-8381-8

Vol. 45 Maurizio Gotti \& Françoise Salager-Meyer (eds)

Advances in Medical Discourse Analysis. Oral and Written Contexts. 492 pages. 2006. ISBN 3-03911-185-X . US-ISBN 0-8204-8382-6 
Vol. 46 Maurizio Gotti \& Susan Šarcević (eds)

Insights into Specialized Translation.

396 pages. 2006. ISBN 3-03911-186-8 · US-ISBN 0-8204-8383-4

Vol. 47 Khurshid Ahmad \& Margaret Rogers (eds)

Evidence-based LSP. Translation, Text and Terminology.

584 pages. 2007. ISBN 978-3-03911-187-9

Vol. 48 Hao Sun \& Dániel Z. Kádár (eds)

It's the Dragon's Turn. Chinese Institutional Discourses.

262 pages. 2008. ISBN 978-3-03911-175-6

Vol. 49 Cristina Suárez-Gómez

Relativization in Early English (950-1250). the Position of Relative Clauses.

149 pages. 2006. ISBN 3-03911-203-1 - US-ISBN 0-8204-8904-2

Vol. 50 Maria Vittoria Calvi \& Luisa Chierichetti (eds)

Nuevas tendencias en el discurso de especialidad.

319 pages. 2006. ISBN 978-3-03911-261-6

Vol. 51 Mari Carmen Campoy \& María José Luzón (eds)

Spoken Corpora in Applied Linguistics.

274 pages. 2008. ISBN 978-3-03911-275-3

Vol. 52 Konrad Ehlich \& Dorothee Heller (Hrsg.)

Die Wissenschaft und ihre Sprachen.

323 pages. 2006. ISBN 978-3-03911-272-2

Vol. 53 Jingyu Zhang

The Semantic Salience Hierarchy Model. The L2 Acquisition of Psych Predicates 273 pages. 2007. ISBN 978-3-03911-300-2

Vol. 54 Norman Fairclough, Giuseppina Cortese \& Patrizia Ardizzone (eds) Discourse and Contemporary Social Change. 555 pages. 2007. ISBN 978-3-03911-276-0

Vol. 55 Jan Engberg, Marianne Grove Ditlevsen, Peter Kastberg \& Martin Stegu (eds) New Directions in LSP Teaching. 331 pages. 2007. ISBN 978-3-03911-433-7

Vol. 56 Dorothee Heller \& Konrad Ehlich (Hrsg.) Studien zur Rechtskommunikation. 322 pages. 2007. ISBN 978-3-03911-436-8

Vol. 57 Teruhiro Ishiguro \& Kang-kwong Luke (eds) Grammar in Cross-Linguistic Perspective.

The Syntax, Semantics, and Pragmatics of Japanese and Chinese. 304 pages. 2012. ISBN 978-3-03911-445-0

Vol. 58 Carmen Frehner

Email - SMS - MMS

294 pages. 2008. ISBN 978-3-03911-451-1

Vol. 59 Isabel Balteiro

The Directionality of Conversion in English. A Dia-Synchronic Study.

276 pages. 2007. ISBN 978-3-03911-241-8

Vol. 60 Maria Milagros Del Saz Rubio

English Discourse Markers of Reformulation.

237 pages. 2007. ISBN 978-3-03911-196-1 
Vol. 61 Sally Burgess \& Pedro Martín-Martín (eds)

English as an Additional Language in Research Publication and Communication. 259 pages. 2008. ISBN 978-3-03911-462-7

Vol. 62 Sandrine Onillon

Pratiques et représentations de l'écrit.

458 pages. 2008. ISBN 978-3-03911-464-1

Vol. 63 Hugo Bowles \& Paul Seedhouse (eds)

Conversation Analysis and Language for Specific Purposes.

$2^{\text {nd }}$ edition. 337 pages. 2007, 2009. ISBN 978-3-0343-0045-2

Vol. 64 Vijay K. Bhatia, Christopher N. Candlin \& Paola Evangelisti Allori (eds)

Language, Culture and the Law.

The Formulation of Legal Concepts across Systems and Cultures.

342 pages. 2008. ISBN 978-3-03911-470-2

Vol. 65 Jonathan Culpeper \& Dániel Z. Kádár (eds)

Historical (Im)politeness.

300 pages. 2010. ISBN 978-3-03911-496-2

Vol. 66 Linda Lombardo (ed.)

Using Corpora to Learn about Language and Discourse.

237 pages. 2009. ISBN 978-3-03911-522-8

Vol. 67 Natsumi Wakamoto

Extroversion/Introversion in Foreign Language Learning.

Interactions with Learner Strategy Use.

159 pages. 2009. ISBN 978-3-03911-596-9

Vol. 68 Eva Alcón-Soler (ed.)

Learning How to Request in an Instructed Language Learning Context.

260 pages. 2008. ISBN 978-3-03911-601-0

Vol. 69 Domenico Pezzini

The Translation of Religious Texts in the Middle Ages.

428 pages. 2008. ISBN 978-3-03911-600-3

Vol. 70 Tomoko Tode

Effects of Frequency in Classroom Second Language Learning.

Quasi-experiment and stimulated-recall analysis.

195 pages. 2008. ISBN 978-3-03911-602-7

Vol. 71 Egor Tsedryk

Fusion symétrique et alternances ditransitives.

211 pages. 2009. ISBN 978-3-03911-609-6

Vol. 72 Cynthia J. Kellett Bidoli \& Elana Ochse (eds)

English in International Deaf Communication.

444 pages. 2008. ISBN 978-3-03911-610-2

Vol. 73 Joan C. Beal, Carmela Nocera \& Massimo Sturiale (eds)

Perspectives on Prescriptivism.

269 pages. 2008. ISBN 978-3-03911-632-4

Vol. 74 Carol Taylor Torsello, Katherine Ackerley \& Erik Castello (eds)

Corpora for University Language Teachers.

308 pages. 2008. ISBN 978-3-03911-639-3 
Vol. 75 María Luisa Pérez Cañado (ed.)

English Language Teaching in the European Credit Transfer System.

Facing the Challenge.

251 pages. 2009. ISBN 978-3-03911-654-6

Vol. 76 Marina Dossena \& Ingrid Tieken-Boon van Ostade (eds)

Studies in Late Modern English Correspondence. Methodology and Data.

291 pages. 2008. ISBN 978-3-03911-658-4

Vol. 77 Ingrid Tieken-Boon van Ostade \& Wim van der Wurff (eds)

Current Issues in Late Modern English.

436 pages. 2009. ISBN 978-3-03911-660-7

Vol. 78 Marta Navarro Coy (ed.)

Practical Approaches to Foreign Language Teaching and Learning.

297 pages. 2009. ISBN 978-3-03911-661-4

Vol. 79 Qing Ma

Second Language Vocabulary Acquisition.

333 pages. 2009. ISBN 978-3-03911-666-9

Vol. 80 Martin Solly, Michelangelo Conoscenti \& Sandra Campagna (eds)

Verbal/Visual Narrative Texts in Higher Education.

384 pages. 2008. ISBN 978-3-03911-672-0

Vol. 81 Meiko Matsumoto

From Simple Verbs to Periphrastic Expressions:

The Historical Development of Composite Predicates, Phrasal Verbs, and Related Constructions in English.

235 pages. 2008. ISBN 978-3-03911-675-1

Vol. 82 Melinda Dooly

Doing Diversity. Teachers' Construction of Their Classroom Reality.

180 pages. 2009. ISBN 978-3-03911-687-4

Vol. 83 Victoria Guillén-Nieto, Carmen Marimón-Llorca \& Chelo Vargas-Sierra (eds) Intercultural Business Communication and

Simulation and Gaming Methodology.

392 pages. 2009. ISBN 978-3-03911-688-1

Vol. 84 Maria Grazia Guido

English as a Lingua Franca in Cross-cultural Immigration Domains.

285 pages. 2008. ISBN 978-3-03911-689-8

Vol. 85 Erik Castello

Text Complexity and Reading Comprehension Tests.

352 pages. 2008. ISBN 978-3-03911-717-8

Vol. 86 Maria-Lluisa Gea-Valor, Isabel García-lzquierdo \& Maria-José Esteve (eds) Linguistic and Translation Studies in Scientific Communication.

317 pages. 2010. ISBN 978-3-0343-0069-8

Vol. 87 Carmen Navarro, Rosa Mª Rodríguez Abella, Francesca Dalle Pezze

\& Renzo Miotti (eds)

La comunicación especializada.

355 pages. 2008. ISBN 978-3-03911-733-8 
Vol. 88 Kiriko Sato

The Development from Case-Forms to Prepositional Constructions

in Old English Prose.

231 pages. 2009. ISBN 978-3-03911-763-5

Vol. 89 Dorothee Heller (Hrsg.)

Formulierungsmuster in deutscher und italienischer Fachkommunikation.

Intra- und interlinguale Perspektiven.

315 pages. 2008. ISBN 978-3-03911-778-9

Vol. 90 Henning Bergenholtz, Sandro Nielsen \& Sven Tarp (eds)

Lexicography at a Crossroads. Dictionaries and Encyclopedias Today,

Lexicographical Tools Tomorrow.

372 pages. 2009. ISBN 978-3-03911-799-4

Vol. 91 Manouchehr Moshtagh Khorasani

The Development of Controversies. From the Early Modern Period

to Online Discussion Forums.

317 pages. 2009. ISBN 978-3-3911-711-6

Vol. 92 María Luisa Carrió-Pastor (ed.)

Content and Language Integrated Learning. Cultural Diversity.

178 pages. 2009. ISBN 978-3-3911-818-2

Vol. 93 Roger Berry

Terminology in English Language Teaching. Nature and Use.

262 pages. 2010. ISBN 978-3-0343-0013-1

Vol. 94 Roberto Cagliero \& Jennifer Jenkins (eds)

Discourses, Communities, and Global Englishes

240 pages. 2010. ISBN 978-3-0343-0012-4

Vol. 95 Facchinetti Roberta, Crystal David, Seidlhofer Barbara (eds)

From International to Local English - And Back Again.

268 pages. 2010. ISBN 978-3-0343-0011-7

Vol. 96 Cesare Gagliardi \& Alan Maley (eds)

EIL, ELF, Global English. Teaching and Learning Issues

376 pages. 2010. ISBN 978-3-0343-0010-0

Vol. 97 Sylvie Hancil (ed.)

The Role of Prosody in Affective Speech.

403 pages. 2009. ISBN 978-3-03911-696-6

Vol. 98 Marina Dossena \& Roger Lass (eds)

Studies in English and European Historical Dialectology.

257 pages. 2009. ISBN 978-3-0343-0024-7

Vol. 99 Christine Béal

Les interactions quotidiennes en français et en anglais.

De l'approche comparative à l'analyse des situations interculturelles.

424 pages. 2010. ISBN 978-3-0343-0027-8

Vol. 100 Maurizio Gotti (ed.)

Commonality and Individuality in Academic Discourse.

398 pages. 2009. ISBN 978-3-0343-0023-0

Vol. 101 Javier E. Díaz Vera \& Rosario Caballero (eds)

Textual Healing. Studies in Medieval English Medical, Scientific and Technical Texts.

213 pages. 2009. ISBN 978-3-03911-822-9 
Vol. 102 Nuria Edo Marzá

The Specialised Lexicographical Approach. A Step further in Dictionary-making. 316 pages. 2009. ISBN 978-3-0343-0043-8

Vol. 103 Carlos Prado-Alonso, Lidia Gómez-García, Iria Pastor-Gómez \&

David Tizón-Couto (eds)

New Trends and Methodologies in Applied English Language Research.

Diachronic, Diatopic and Contrastive Studies.

348 pages. 2009. ISBN 978-3-0343-0046-9

Vol. 104 Françoise Salager-Meyer \& Beverly A. Lewin

Crossed Words. Criticism in Scholarly Writing?

371 pages. 2011. ISBN 978-3-0343-0049-0.

Vol. 105 Javier Ruano-García

Early Modern Northern English Lexis. A Literary Corpus-Based Study.

611 pages. 2010. ISBN 978-3-0343-0058-2

Vol. 106 Rafael Monroy-Casas

Systems for the Phonetic Transcription of English. Theory and Texts.

280 pages. 2011. ISBN 978-3-0343-0059-9

Vol. 107 Nicola T. Owtram

The Pragmatics of Academic Writing.

A Relevance Approach to the Analysis of Research Article Introductions.

311 pages. 2009. ISBN 978-3-0343-0060-5

Vol. 108 Yolanda Ruiz de Zarobe, Juan Manuel Sierra \&

Francisco Gallardo del Puerto (eds)

Content and Foreign Language Integrated Learning.

Contributions to Multilingualism in European Contexts

343 pages. 2011. ISBN 978-3-0343-0074-2

Vol. 109 Ángeles Linde López \& Rosalía Crespo Jiménez (eds)

Professional English in the European context. The EHEA challenge.

374 pages. 2010. ISBN 978-3-0343-0088-9

Vol. 110 Rosalía Rodríguez-Vázquez

The Rhythm of Speech, Verse and Vocal Music. A New Theory.

394 pages. 2010. ISBN 978-3-0343-0309-5

Vol. 111 Anastasios Tsangalidis \& Roberta Facchinetti (eds)

Studies on English Modality. In Honour of Frank Palmer.

392 pages. 2009. ISBN 978-3-0343-0310-1

Vol. 112 Jing Huang

Autonomy, Agency and Identity in Foreign Language Learning and Teaching. 400 pages. 2013. ISBN 978-3-0343-0370-5

Vol. 113 Mihhail Lotman \& Maria-Kristiina Lotman (eds)

Frontiers in Comparative Prosody. In memoriam: Mikhail Gasparov.

426 pages. 2011. ISBN 978-3-0343-0373-6

Vol. 114 Merja Kytö, John Scahill \& Harumi Tanabe (eds)

Language Change and Variation from Old English to Late Modern English.

A Festschrift for Minoji Akimoto

422 pages. 2010. ISBN 978-3-0343-0372-9

Vol. 115 Giuliana Garzone \& Paola Catenaccio (eds)

Identities across Media and Modes. Discursive Perspectives.

379 pages. 2009. ISBN 978-3-0343-0386-6 
Vol. 116 Elena Landone

Los marcadores del discurso y cortesía verbal en español.

390 pages. 2010. ISBN 978-3-0343-0413-9

Vol. 117 Maurizio Gotti \& Christopher Williams (eds)

Legal Discourse across Languages and Cultures.

339 pages. 2010. ISBN 978-3-0343-0425-2

Vol. 118 David Hirsh

Academic Vocabulary in Context.

217 pages. 2010. ISBN 978-3-0343-0426-9

Vol. 119 Yvonne Dröschel

Lingua Franca English. The Role of Simplification and Transfer.

358 pages. 2011. ISBN 978-3-0343-0432-0

Vol. 120 Tengku Sepora Tengku Mahadi, Helia Vaezian \& Mahmoud Akbari

Corpora in Translation. A Practical Guide.

135 pages. 2010. ISBN 978-3-0343-0434-4

Vol. 121 Davide Simone Giannoni \& Celina Frade (eds)

Researching Language and the Law. Textual Features and Translation Issues.

278 pages. 2010. ISBN 978-3-0343-0443-6

Vol. 122 Daniel Madrid \& Stephen Hughes (eds)

Studies in Bilingual Education.

472 pages. 2011. ISBN 978-3-0343-0474-0

Vol. 123 Vijay K. Bhatia, Christopher N. Candlin \& Maurizio Gotti (eds)

The Discourses of Dispute Resolution.

290 pages. 2010. ISBN 978-3-0343-0476-4

Vol. 124 Davide Simone Giannoni

Mapping Academic Values in the Disciplines. A Corpus-Based Approach.

288 pages. 2010. ISBN 978-3-0343-0488-7

Vol. 125 Giuliana Garzone \& James Archibald (eds)

Discourse, Identities and Roles in Specialized Communication.

419 pages. 2010. ISBN 978-3-0343-0494-8

Vol. 126 Iria Pastor-Gómez

The Status and Development of $\mathrm{N}+\mathrm{N}$ Sequences in

Contemporary English Noun Phrases.

216 pages. 2011. ISBN 978-3-0343-0534-1

Vol. 127 Carlos Prado-Alonso

Full-verb Inversion in Written and Spoken English.

261 pages. 2011. ISBN 978-3-0343-0535-8

Vol. 128 Tony Harris \& María Moreno Jaén (eds)

Corpus Linguistics in Language Teaching.

214 pages. 2010. ISBN 978-3-0343-0524-2

Vol. 129 Tetsuji Oda \& Hiroyuki Eto (eds)

Multiple Perspectives on English Philology and History of Linguistics.

A Festschrift for Shoichi Watanabe on his $80^{\text {th }}$ Birthday.

378 pages. 2010. ISBN 978-3-0343-0480-1 
Vol. 130 Luisa Chierichetti \& Giovanni Garofalo (eds)

Lengua y Derecho. líneas de investigación interdisciplinaria.

283 pages. 2010. 978-3-0343-0463-4

Vol. 131 Paola Evangelisti Allori \& Giuliana Garzone (eds)

Discourse, Identities and Genres in Corporate Communication.

Sponsorship, Advertising and Organizational Communication.

324 pages. 2011. 978-3-0343-0591-4

Vol. 132 Leyre Ruiz de Zarobe \& Yolanda Ruiz de Zarobe (eds)

Speech Acts and Politeness across Languages and Cultures.

402 pages. 2012. 978-3-0343-0611-9

Vol. 133 Thomas Christiansen

Cohesion. A Discourse Perspective.

387 pages. 2011. 978-3-0343-0619-5

Vol. 134 Giuliana Garzone \& Maurizio Gotti

Discourse, Communication and the Enterprise. Genres and Trends.

451 pages. 2011. ISBN 978-3-0343-0620-1

Vol. 135 Zsuzsa Hoffmann

Ways of the World's Words.

Language Contact in the Age of Globalization.

334 pages 2011. ISBN 978-3-0343-0673-7

Vol. 136 Cecilia Varcasia (ed.)

Becoming Multilingual.

Language Learning and Language Policy between Attitudes and Identities.

213 pages. 2011. ISBN 978-3-0343-0687-5

Vol. 137 Susy Macqueen

The Emergence of Patterns in Second Language Writing.

A Sociocognitive Exploration of Lexical Trails.

325 pages. 2012. ISBN 978-3-0343-1010-9

Vol. 138 Maria Vittoria Calvi \& Giovanna Mapelli (eds)

La lengua del turismo. Géneros discursivos y terminología.

365 pages. 2011. ISBN 978-3-0343-1011-6

Vol. 139 Ken Lau

Learning to Become a Professional in a Textually-Mediated World.

A Text-Oriented Study of Placement Practices.

261 pages. 2012. ISBN 978-3-0343-1016-1

Vol. 140 Sandra Campagna, Giuliana Garzone, Cornelia Ilie \& Elizabeth Rowley-Jolivet (eds)

Evolving Genres in Web-mediated Communication.

337 pages. 2012. ISBN 978-3-0343-1013-0

Vol. 141 Edith Esch \& Martin Solly (eds)

The Sociolinguistics of Language Education in International Contexts.

263 pages. 2012. ISBN 978-3-0343-1009-3

Vol. 142 Forthcoming.

Vol. 143 David Tizón-Couto

Left Dislocation in English. A Functional-Discoursal Approach.

416 pages. 2012. ISBN 978-3-0343-1037-6 
Vol. 144 Margrethe Petersen \& Jan Engberg (eds)

Current Trends in LSP Research. Aims and Methods.

323 pages. 2011. ISBN 978-3-0343-1054-3

Vol. 145 David Tizón-Couto, Beatriz Tizón-Couto, Iria Pastor-Gómez \& Paula Rodríguez-Puente (eds)

New Trends and Methodologies in Applied English Language Research II.

Studies in Language Variation, Meaning and Learning.

283 pages. 2012. ISBN 978-3-0343-1061-1

Vol. 146 Rita Salvi \& Hiromasa Tanaka (eds)

Intercultural Interactions in Business and Management.

306 pages. 2011. ISBN 978-3-0343-1039-0

Vol. 147 Francesco Straniero Sergio \& Caterina Falbo (eds)

Breaking Ground in Corpus-based Interpreting Studies.

254 pages. 2012. ISBN 978-3-0343-1071-0

Vol. 148 Forthcoming.

Vol. 149 Vijay K. Bhatia \& Paola Evangelisti Allori (eds)

Discourse and Identity in the Professions. Legal, Corporate and Institutional Citizenship. 352 pages. 2011. ISBN 978-3-0343-1079-6

Vol. 150 Maurizio Gotti (ed.)

Academic Identity Traits. A Corpus-Based Investigation.

363 pages. 2012. ISBN 978-3-0343-1141-0

Vol. 151 Priscilla Heynderickx, Sylvain Dieltjens, Geert Jacobs, Paul Gillaerts \&

Elizabeth de Groot (eds)

The Language Factor in International Business.

New Perspectives on Research, Teaching and Practice.

320 pages. 2012. ISBN 978-3-0343-1090-1

Vol. 152 Paul Gillaerts, Elizabeth de Groot, Sylvain Dieltjens, Priscilla Heynderickx \&

Geert Jacobs (eds)

Researching Discourse in Business Genres. Cases and Corpora.

215 pages. 2012. ISBN 978-3-0343-1092-5

Vol. 153 Yongyan Zheng

Dynamic Vocabulary Development in a Foreign Language.

262 pages. 2012. ISBN 978-3-0343-1106-9

Vol. 154 Carmen Argondizzo (ed.)

Creativity and Innovation in Language Education.

357 pages. 2012. ISBN 978-3-0343-1080-2

Vol. 155 David Hirsh (ed.)

Current Perspectives in Second Language Vocabulary Research. 180 pages. 2012. ISBN 978-3-0343-1108-3

Vol. 156 Seiji Shinkawa

Unhistorical Gender Assignment in Lazamon's Brut. A Case Study of a Late Stage in the Development of Grammatical Gender toward its Ultimate Loss.

186 pages. 2012. ISBN 978-3-0343-1124-3

Vol. 157 Yeonkwon Jung

Basics of Organizational Writing: A Critical Reading Approach.

151 pages. 2014. ISBN 978-3-0343-1137-3. 
Vol. 158 Bárbara Eizaga Rebollar (ed.)

Studies in Linguistics and Cognition.

301 pages. 2012. ISBN 978-3-0343-1138-0

Vol. 159 Giuliana Garzone, Paola Catenaccio, Chiara Degano (eds)

Genre Change in the Contemporary World. Short-term Diachronic Perspectives. 329 pages. 2012. ISBN 978-3-0343-1214-1

Vol. 160 Carol Berkenkotter, Vijay K. Bhatia \& Maurizio Gotti (eds)

Insights into Academic Genres.

468 pages. 2012. ISBN 978-3-0343-1211-0

Vol. 161 Beatriz Tizón-Couto

Clausal Complements in Native and Learner Spoken English. A corpus-based study with Lindsei and Vicolse. 357 pages. 2013. ISBN 978-3-0343-1184-7

Vol. 162 Patrizia Anesa

Jury Trials and the Popularization of Legal Language. A Discourse Analytical Approach. 247 pages. 2012. ISBN 978-3-0343-1231-8

Vol. 163 David Hirsh

Endangered Languages, Knowledge Systems and Belief Systems.

153 pages. 2013. ISBN 978-3-0343-1232-5

Vol. 164 Eugenia Sainz (ed.)

De la estructura de la frase al tejido del discurso. Estudios contrastivos español/italiano. 305 pages. 2014. ISBN 978-3-0343-1253-0

Vol. 165 Julia Bamford, Franca Poppi \& Davide Mazzi (eds)

Space, Place and the Discursive Construction of Identity.

367 pages. 2014. ISBN 978-3-0343-1249-3

Vol. 166 Rita Salvi \& Janet Bowker (eds)

Space, Time and the Construction of Identity.

Discursive Indexicality in Cultural, Institutional and Professional Fields.

324 pages. 2013. ISBN 978-3-0343-1254-7

Vol. 167 Shunji Yamazaki \& Robert Sigley (eds)

Approaching Language Variation through Corpora. A Festschrift in Honour of Toshio Saito. 421 pages. 2013. ISBN 978-3-0343-1264-6

Vol. 168 Franca Poppi

Global Interactions in English as a Lingua Franca. How written communication is changing under the influence of electronic media and new contexts of use.

249 pages. 2012. ISBN 978-3-0343-1276-9

Vol. 169 Miguel A. Aijón Oliva \& María José Serrano

Style in syntax. Investigating variation in Spanish pronoun subjects.

239 pages. 2013. ISBN 978-3-0343-1244-8

Vol. 170 Inés Olza, Óscar Loureda \& Manuel Casado-Velarde (eds)

Language Use in the Public Sphere. Methodological Perspectives and Empirical Applications 564 pages. 2014. ISBN 978-3-0343-1286-8

Vol. 171 Aleksandra Matulewska

Legilinguistic Translatology. A Parametric Approach to Legal Translation.

279 pages. 2013. ISBN 978-3-0343-1287-5

Vol. 172 Maurizio Gotti \& Carmen Sancho Guinda (eds)

Narratives in Academic and Professional Genres.

513 pages. 2013. ISBN 978-3-0343-1371-1 
Vol. 173 Madalina Chitez

Learner corpus profiles. The case of Romanian Learner English.

244 pages. 2014. ISBN 978-3-0343-1410-7

Vol. 174 Chihiro Inoue

Task Equivalence in Speaking Tests.

251 pages. 2013. ISBN 978-3-0343-1417-6

Vol. 175 Gabriel Quiroz \& Pedro Patiño (eds.)

LSP in Colombia: advances and challenges.

339 pages. 2014. ISBN 978-3-0343-1434-3

Vol. 176 Catherine Resche

Economic Terms and Beyond: Capitalising on the Wealth of Notions.

How Researchers in Specialised Varieties of English Can Benefit from Focusing on Terms. 332 pages. 2013. ISBN 978-3-0343-1435-0

Vol. 177 Forthcoming.

Vol. 178 Cécile Desoutter \& Caroline Mellet (dir.)

Le discours rapporté: approches linguistiques et perspectives didactiques.

270 pages. 2013. ISBN 978-3-0343-1292-9

Vol. 179 Ana Díaz-Negrillo \& Francisco Javier Díaz-Pérez (eds)

Specialisation and Variation in Language Corpora.

341 pages. 2014. ISBN 978-3-0343-1316-2

Vol. 180 Pilar Alonso

A Multi-dimensional Approach to Discourse Coherence. From Standardness to Creativity. 247 pages. 2014. ISBN 978-3-0343-1325-4

Vol. 181 Alejandro Alcaraz-Sintes \& Salvador Valera-Hernández (eds)

Diachrony and Synchrony in English Corpus Linguistics.

393 pages. 2014. ISBN 978-3-0343-1326-1

Vol. 182 Runhan Zhang

Investigating Linguistic Knowledge of a Second Language.

207 pages. 2015. ISBN 978-3-0343-1330-8

Vol. 183 Hajar Abdul Rahim \& Shakila Abdul Manan (eds.)

English in Malaysia. Postcolonial and Beyond.

267 pages. 2014. ISBN 978-3-0343-1341-4

Vol. 184 Virginie Fasel Lauzon

Comprendre et apprendre dans l'interaction. Les séquences d'explication en classe de français langue seconde.

292 pages. 2014. ISBN 978-3-0343-1451-0

Vol. 185 Forthcoming.

Vol. 186 Wei Ren

L2 Pragmatic Development in Study Abroad Contexts

256 pages. 2015. ISBN 978-3-0343-1358-2

Vol. 187 Marina Bondi \& Rosa Lorés Sanz (eds)

Abstracts in Academic Discourse. Variation and Change.

361 pages. 2014. ISBN 978-3-0343-1483-1

Vol. 188 Giuditta Caliendo

Rethinking Community. Discourse, Identity and Citizenship in the European Union. 258 pages. 2017. ISBN 978-3-0343-1561-6 
Vol. 189 Paola Evangelisti Allori (ed.)

Identities in and across Cultures.

315 pages. 2014. ISBN 978-3-0343-1458-9

Vol. 190 Erik Castello, Katherine Ackerley \& Francesca Coccetta (eds).

Studies in Learner Corpus Linguistics. Research and Applications for Foreign Language Teaching and Assessment.

358 pages. 2015. ISBN 978-3-0343-1506-7

Vol. 191 Ruth Breeze, Maurizio Gotti \& Carmen Sancho Guinda (eds)

Interpersonality in Legal Genres.

389 pages. 2014. ISBN 978-3-0343-1524-1

Vol. 192 Paola Evangelisti Allori, John Bateman \& Vijay K. Bhatia (eds)

Evolution in Genre. Emergence, Variation, Multimodality.

364 pages. 2014. ISBN 978-3-0343-1533-3

Vol. 193 Jiyeon Kook

Agency in Arzt-Patient-Gesprächen. Zur interaktionistischen Konzeptualisierung von Agency 271 pages. 2015. ISBN 978-3-0343-1666-8

Vol. 194 Susana Nicolás Román \& Juan José Torres Núñez (eds)

Drama and CLIL. A new challenge for the teaching approaches in bilingual education.

170 pages. 2015. ISBN 978-3-0343-1629-3

Vol. 195 Alessandra Molino \& Serenella Zanotti (eds)

Observing Norm, Observing Usage. Lexis in Dictionaries and in the Media.

430 pages. 2015. ISBN 978-3-0343-1584-5

Vol. 196 Begoña Soneira

A Lexical Description of English for Architecture. A Corpus-based Approach.

267 pages. 2015. ISBN 978-3-0343-1602-6

Vol. 197 M Luisa Roca-Varela

False Friends in Learner Corpora. A corpus-based study of English false friends

in the written and spoken production of Spanish learners.

348 pages. 2015. ISBN 978-3-0343-1620-0

Vol. 198 Rahma Al-Mahrooqi \& Christopher Denman

Bridging the Gap between Education and Employment. English Language Instruction in EFL Contexts.

416 pages. 2015. ISBN 978-3-0343-1681-1

Vol. 199 Rita Salvi \& Janet Bowker (eds)

The Dissemination of Contemporary Knowledge in English. Genres, discourse strategies and professional practices.

171 pages. 2015. ISBN 978-3-0343-1679-8

Vol. 200 Maurizio Gotti \& Davide S. Giannoni (eds)

Corpus Analysis for Descriptive and Pedagogical Purposes. ESP Perspectives.

432 pages. 2014. ISBN 978-3-0343-1516-6

Vol. 201 Ida Ruffolo

The Perception of Nature in Travel Promotion Texts. A Corpus-based Discourse Analysis. 148 pages. 2015. ISBN 978-3-0343-1521-0

Vol. 202 Ives Trevian

English suffixes. Stress-assignment properties, productivity, selection and combinatorial processes.

471 pages. 2015. ISBN 978-3-0343-1576-0 
Vol. 203 Maurizio Gotti, Stefania Maci \& Michele Sala (eds)

Insights into Medical Communication.

422 pages. 2015. ISBN 978-3-0343-1694-1

Vol. 204 Carmen Argondizzo (ed.)

European Projects in University Language Centres. Creativity, Dynamics, Best Practice. 371 pages. 2015. ISBN 978-3-0343-1696-5

Vol. 205 Aura Luz Duffé Montalván (ed.)

Estudios sobre el léxico. Puntos y contrapuntos.

502 pages. 2016. ISBN 978-3-0343-2011-5

Vol. 206 Maria Pavesi, Maicol Formentelli \& Elisa Ghia (eds)

The Languages of Dubbing. Mainstream Audiovisual Translation in Italy.

275 pages. 2014. ISBN 978-3-0343-1646-0

Vol. 207 Ruth Breeze \& Inés Olza (eds)

Evaluation in media discourse. European perspectives.

268 pages. 2017. ISBN 978-3-0343-2014-6

Vol. 208 Vijay K. Bhatia \& Maurizio Gotti (eds)

Arbitration Discourse in Asia.

331 pages. 2015. ISBN 978-3-0343-2032-0

Vol. 209 Sofía Bemposta-Rivas, Carla Bouzada-Jabois, Yolanda Fernández-Pena,

Tamara Bouso, Yolanda J. Calvo-Benzies, Iván Tamaredo (eds)

New trends and methodologies in applied English language research III.

Synchronic and diachronic studies on discourse, lexis and grammar processing.

280 pages. 2017. ISBN 978-3-0343-2039-9

Vol. 210 Francisco Alonso Almeida, Laura Cruz García \& Víctor González Ruiz (eds)

Corpus-based studies on language varieties.

285 pages. 2016. ISBN 978-3-0343-2044-3

Vol. 211 Juan Pedro Rica Peromingo

Aspectos lingüísticos y técnicos de la traducción audiovisual (TAV).

177 pages. 2016. ISBN 978-3-0343-2055-9

Vol. 212 Maria Vender

Disentangling Dyslexia. VenderPhonological and Processing Deficit in

Developmental Dyslexia.

338 pages. 2017. ISBN 978-3-0343-2064-1

Vol. 213 Zhilong Xie

Bilingual Advantages. Contributions of Different Bilingual Experiences to Cognitive

Control Differences Among Young-adult Bilinguals.

221 pages. 2016. ISBN 978-3-0343-2081-8

Vol. 214 Larissa D’Angelo

Academic posters. A textual and visual metadiscourse analysis.

367 pages. 2016. ISBN 978-3-0343-2083-2

Vol. 215 Evelyne Berger

Prendre la parole en L2. Regard sur la compétence d'interaction en classe de langue. 246 pages. 2016. ISBN 978-3-0343-2084-9

Vol. 216 David Lasagabaster and Aintzane Doiz (eds)

CLIL experiences in secondary and tertiary education: In search of good practices.

262 pages. 2016. ISBN 978-3-0343-2104-4 
Vol. 217 Elena Kkese

Identifying Plosives in L2 English: The Case of L1 Cypriot Greek Speakers.

317 pages. 2016. ISBN 978-3-0343-2060-3

Vol. 218 Sandra Campagna, Elana Ochse, Virginia Pulcini \& Martin Solly (eds)

Languaging in and across Communities: New Voices, New Identities. Studies in Honour of Giuseppina Cortese.

507 pages. 2016. ISBN 978-3-0343-2073-3

Vol. 219 Adriana Orlandi \& Laura Giacomini (ed.)

Defining collocation for lexicographic purposes. From linguistic theory to lexicographic practice.

328 pages. 2016. ISBN 978-3-0343-2054-2

Vol. 220 Pietro Luigi laia

Analysing English as a Lingua Franca in Video Games. Linguistic Features,

Experiential and Functional Dimensions of Online and Scripted Interactions.

139 pages. 2016. ISBN 978-3-0343-2138-9

Vol. 221 Dimitrinka G. Níkleva (ed.)

La formación de los docentes de español para inmigrantes en distintos contextos

educativos.

390 pages. 2017. ISBN 978-3-0343-2135-8

Vol. 222 Katherine Ackerley, Marta Guarda \& Francesca Helm (eds)

Sharing Perspectives on English-Medium Instruction.

308 pages. 2017. ISBN 978-3-0343-2537-0

Vol. 223 Juana I. Marín-Arrese, Julia Lavid-López, Marta Carretero, Elena Domínguez Romero, $M^{a}$ Victoria Martín de la Rosa \& María Pérez Blanco (eds)

Evidentiality and Modality in European Languages. Discourse-pragmatic perspectives. 427 pages. 2017. ISBN 978-3-0343-2437-3

Vol. 224 Gilles Col

Construction du sens : un modèle instructionnel pour la sémantique.

292 pages. 2017. ISBN 978-3-0343-2572-1

Vol. 225 Ana Chiquito \& Gabriel Quiroz (eds)

Pobreza, Lenguaje y Medios en América Latina.

362 pages. 2017. ISBN 978-3-0343-2142-6

Vol. 226 Xu Zhang

English Quasi-Numeral Classifiers. A Corpus-Based Cognitive-Typological Study. 360 pages. 2017. ISBN 978-3-0343-2818-0

Vol. 227 María Ángeles Orts, Ruth Breeze \& Maurizio Gotti (eds)

Power, Persuasion and Manipulation in Specialised Genres. Providing Keys to the Rhetoric of Professional Communities.

368 pages. 2017. ISBN 978-3-0343-3010-7

Vol. 228 Maurizio Gotti, Stefania Maci \& Michele Sala (eds)

Ways of Seeing, Ways of Being: Representing the Voices of Tourism.

453 pages. 2017. ISBN 978-3-0343-3031-2

Vol. 229 Forthcoming.

Vol. 230 Anca-Cristina Sterie

Interprofessional interactions at the hospital. Nurses' requests and reports

of problems in calls with physicians.

371 pages. 2017. ISBN 978-3-0343-2734-3 
Vol. 231 Xiaodong Zhang

Understanding Chinese EFL Teachers' Beliefs and Practices in the Textbook-Based Classroom. 189 pages. 2017. ISBN 978-3-0343-3053-4

Vol. 232 Manuela Caterina Moroni \& Federica Ricci Garotti (Hrsg.)

Brücken schlagen zwischen Sprachwissenschaft und DaF-Didaktik.

345 pages. 2017. ISBN 978-3-0343-2667-4

Vol. 233 Forthcoming.

Vol. 234 Forthcoming.

Vol. 235 Forthcoming.

Vol. 236 Maria Chiara Janner

Sguardi linguistici sulla marca. Analisi morfosintattica dei nomi commerciali in italiano 364 pages. 2017. ISBN 978-3-0343-2667-4

Vol. 237 Bárbara Herrero Muñoz-Cobo \& Otman El Azami Zalachi

La primavera del árabe marroquí.

192 pages. 2017. ISBN 978-3-0343-3104-3

Vol. 238 Forthcoming.

Vol. 239 Stefania M. Maci

The MS Digby 133 «Mary Magdalene». Beyond scribal practices: language, discourse, values and attitudes.

336 pages. 2017. ISBN 978-3-0343-3256-9 